\title{
Mitigation Studies of the Airfoil-Vortex Interaction Using Large Eddy Simulation
}

\author{
By \\ James Daniel Falconer \\ A thesis submitted to \\ The Faculty of Graduate Studies and Research \\ in partial fulfilment of \\ the degree requirements of \\ Master of Applied Science \\ in Aerospace Engineering \\ Ottawa-Carleton Institute for \\ Mechanical and Aerospace Engineering \\ Department of Mechanical and Aerospace Engineering \\ Carleton University \\ Ottawa, Ontario, Canada \\ September 2009 \\ Copyright (C) \\ 2009 - James Daniel Falconer
}




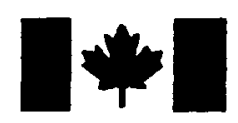

Library and Archives Canada

Published Heritage

Branch

395 Wellington Street Ottawa ON K1A ON4 Canada
Bibliothèque et

Archives Canada

Direction du

Patrimoine de l'édition

395 , rue Wellington

Ottawa ON K1A ON4

Canada
Your file Votre référence
ISBN: 978-0-494-60261-4
Our file Notre référence
ISBN: 978-0-494-60261-4

\section{NOTICE:}

The author has granted a nonexclusive license allowing Library and Archives Canada to reproduce, publish, archive, preserve, conserve, communicate to the public by telecommunication or on the Internet, loan, distribute and sell theses worldwide, for commercial or noncommercial purposes, in microform, paper, electronic and/or any other formats.

The author retains copyright ownership and moral rights in this thesis. Neither the thesis nor substantial extracts from it may be printed or otherwise reproduced without the author's permission.
AVIS:

L'auteur a accordé une licence non exclusive permettant à la Bibliothèque et Archives Canada de reproduire, publier, archiver, sauvegarder, conserver, transmettre au public par télécommunication ou par l'Internet, prêter, distribuer et vendre des thèses partout dans le monde, à des fins commerciales ou autres, sur support microforme, papier, électronique et/ou autres formats.

L'auteur conserve la propriété du droit d'auteur et des droits moraux qui protège cette thèse. $\mathrm{Ni}$ la thèse ni des extraits substantiels de celle-ci ne doivent être imprimés ou autrement reproduits sans son autorisation.
In compliance with the Canadian Privacy Act some supporting forms may have been removed from this thesis.

While these forms may be included in the document page count, their removal does not represent any loss of content from the thesis.
Conformément à la loi canadienne sur la protection de la vie privée, quelques formulaires secondaires ont été enlevés de cette thèse.

Bien que ces formulaires aient inclus dans la pagination, il n'y aura aucun contenu manquant.

\section{Canadä}




\section{Abstract}

Numerical investigations of the mitigation of airfoil-vortex interactions (AVI) using Large Eddy Simulation (LES) were performed. Novel passages between the upper and lower surfaces of the airfoil mitigated AVIs. A NACA 0012 airfoil at zero angle of attack and the Smagorinsky sub-grid scale model with Van Driest Damping function were used as a benchmark airfoil. The freestream Reynolds number was 300,000 based on the airfoil chord $(c=0.46 \mathrm{~m})$. The Mach number was $M=0.036$. The vortex size had strength of $0.9 \mathrm{~m}^{2} / \mathrm{s}$ and a viscous core radius of $0.023 \mathrm{~m}$, following the Lamb-Oseen model. Miss-distance was $y_{v}=-0.067 \mathrm{~m}$. The LES AVI results (lift and moment coefficients) for no passage were validated against experimental data and verified against URANS (Unsteady Reynolds Averaged Navier-Stokes) simulation (using a Shear Stress Transport turbulence model). Simulations for a vertical passage (placed a $1 / 4$ chord location, with a width of $0.05 \mathrm{c})$, an inclined $45^{\circ}$ passage $\left(y_{v}=-0.067 \mathrm{~m}\right)$, and inclined $45^{\circ}$ passage $\left(y_{v}=0 \mathrm{~m}\right)$ were performed with LES. Finally the inclined $45^{\circ}$ passage was simulated with LES at $M=0.25$, a vortex strength of $6.3 \mathrm{~m}^{2} / \mathrm{s}$ and $y_{v}=0 \mathrm{~m}$. Each measured the mitigation of the interaction against the NACA 0012 results with incremental lift coefficient (lift spike) as mitigation. Simulation results indicate mitigation (upwards of $5.3 \%$ reduction in the incremental lift coefficient) of lift and moment coefficients for the $45^{\circ}$ passage when compared against the benchmark airfoil. 
There was a noticeable penalty in drag throughout the interaction for the $45^{\circ}$ passage. If the Courant number is lower than unity for URANS of AVI, lift/moment predictions with the same computational time as LES are reasonable. LES was determined to be a valid method for BVI investigations. 
I dedicate this work to my grandparents: Elaine and Frank Doyle, and Gladys and Ron Falconer. 


\section{Acknowledgements}

I would like to acknowledge my co-supervisors through my Master's degree, Dr. Edgar Matida and Dr. Fred Nitzsche from the Department of Mechanical and Aerospace Engineering at Carleton University. I am very grateful for the opportunity to work with each of them on this project as well as for the use of their computing equipment to ensure a swift and conclusive finish to the work. Furthermore, I am very thankful for their financial support throughout the duration of my graduate studies.

Also, I would like to thank my sister, Megan Falconer, for editing the entire document no matter how tedious the task seemed. I would like to acknowledge my father, Andrew Falconer, who allowed me to stay for two more years so I could focus on my studies. I would also like to acknowledge the rest of my family, Joanne, Matt, and Ben Falconer as well as my grandparents, for their love and support throughout the past two years.

Last, but certainly not least, I would like to acknowledge my friends for supporting me throughout the past two years as well; you know who you are. 


\section{Table of Contents}

$\begin{array}{ll}\text { Abstract } & \text { iii }\end{array}$

$\begin{array}{lll}\text { Acknowledgements } & \text { vi }\end{array}$

$\begin{array}{ll}\text { Table of Contents } & \text { vii }\end{array}$

List of Tables $\quad$ xi

List of Figures $\quad$ xii

$\begin{array}{ll}\text { Nomenclature } & \text { xvii }\end{array}$

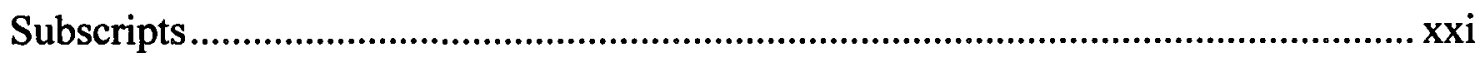

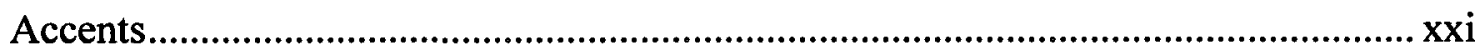

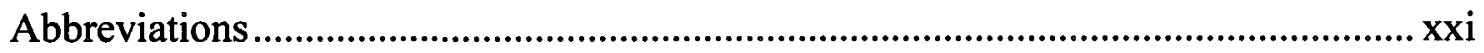

Chapter 1: Introduction and Motivation 1

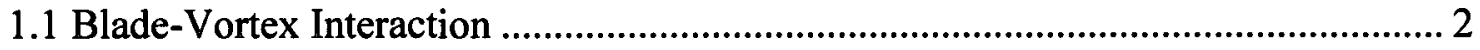

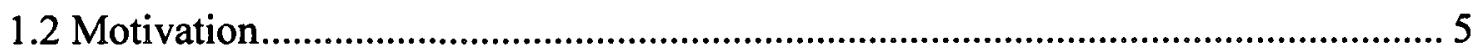

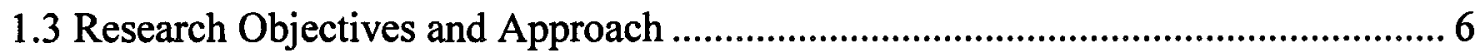

$\begin{array}{ll}\text { Chapter 2: Blade-Vortex Interactions } & 7\end{array}$

2.1 Aerodynamics of the Blade-Vortex Interaction ................................................ 17

2.2 Aeroacoustics of the Blade-Vortex Interaction.................................................. 24

2.3 Investigations Concerning the Blade-Vortex Interaction .................................... 33

2.3.1 Experimental Investigations in Blade (Airfoil)-Vortex Interaction................ 34

2.3.2 Investigations Concerning the Reduction of the Noise Produced by a Blade-

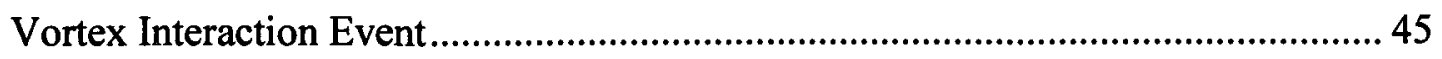

2.3.3 Numerical Investigations in Blade (Airfoil)-Vortex Interaction .....................50 
Chapter 3: Computational Methods for Investigating Airfoil-Vortex Interactions in

Turbulent Flows

3.1 Reynolds Averaged Navier-Stokes (RANS) Simulation ........................................ 58

3.1.1 Governing Equations for RANS ..................................................................5 58

3.1.2 Shear Stress Transport (SST) Model ........................................................... 59

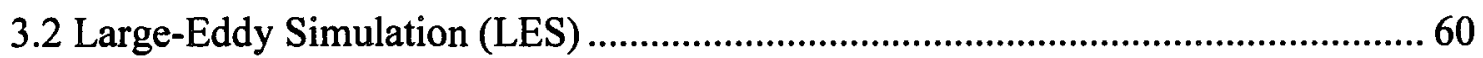

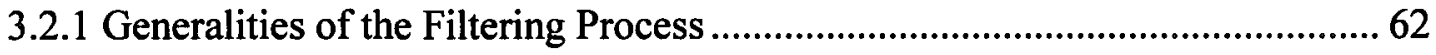

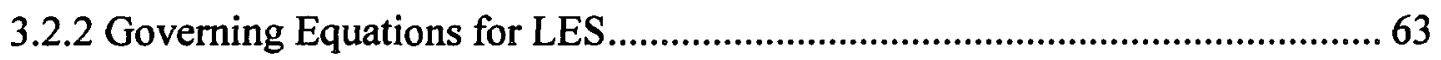

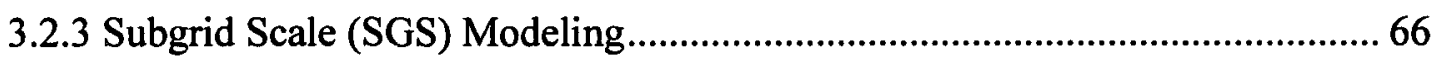

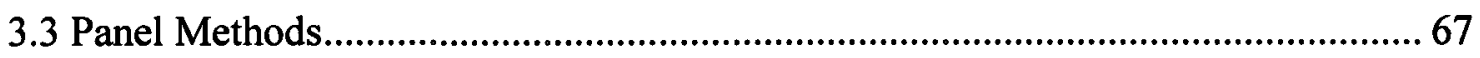

3.3.1 Constant Strength Vortex Panel Method ................................................................ 68

3.3.2 Discrete Vortex Method........................................................................................ 72

Chapter 4: Blade Tip Vortices $\quad \mathbf{7 4}$

4.1 Aerodynamic Definitions Related to Blade Tip Vortices .......................................... 78

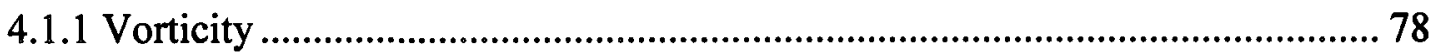

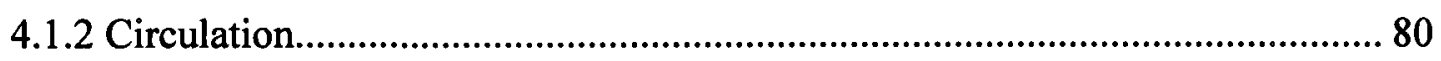

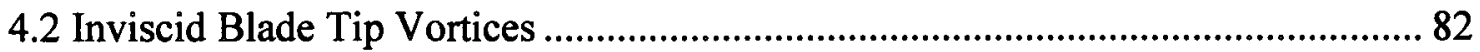

4.3 Viscous Blade Tip Vortices .............................................................................. 84

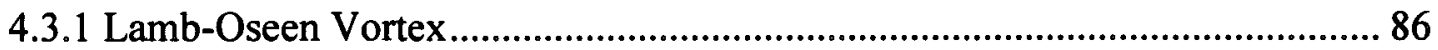

4.3.2 Experimental and Numerical Investigations Related to Viscous Vortices and

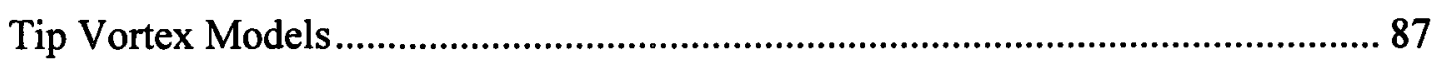

4.4 Diffusion of Blade Tip Vortices ........................................................................... 90

4.4.1 Laminar Models of Tip Vortex Diffusion....................................................... 92

4.4.2 Turbulent Models of Tip Vortex Diffusion ..................................................... 93

4.4.3 Further Investigations Related to Blade Tip Vortex Diffusion......................... 97

Chapter 5: Aerodynamics of the Airfoil-Vortex Interaction I: Verification and Validation of the Computational Method 99

5.1 Governing Equations and Computational Resources Employed ........................... 100 viii 
5.2 Computational and Fluid Models.

5.3 Computational Domains ................................................................................. 102

5.4 Boundary Conditions and Initialization ............................................................. 106

5.4.1 Further Details of the Analysis Performed by Straus et al. (1990)................ 106

5.4.2 Initialization of the Computational Domain .................................................... 111

5.4.3 Boundary Conditions Imposed on the Computational Domains .................... 114

5.5 Definitions of the Aerodynamic Coefficients ...................................................... 115

5.6 Grid Convergence Study ............................................................................... 116

5.7 Domain Convergence Study ............................................................................ 121

5.8 Verification of the Computational Method......................................................... 125

5.9 Validation of the use of LES for Blade-Vortex Interaction Simulations............... 133

Chapter 6: Aerodynamics of the Airfoil-Vortex Interaction II: Mitigation of the $\begin{array}{ll}\text { Airfoil-Vortex Interaction } & 140\end{array}$

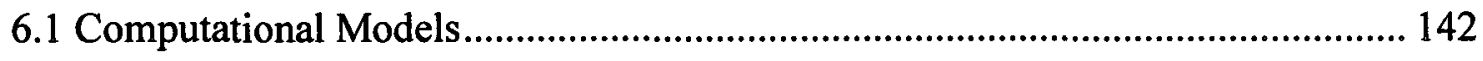

6.2 Computational Domains .................................................................................... 144

6.3 Boundary Conditions and Vortex Initialization .................................................... 148

6.3.1 Initialization of the Vortices for the Mitigation Studies ................................ 148

6.3.2 Boundary Conditions Imposed on the Computational Domains ................... 150

6.4 Quantifying the Mitigation of the Airfoil-Vortex Interaction................................. 152

6.5 Mitigation Study 1 ........................................................................................ 154

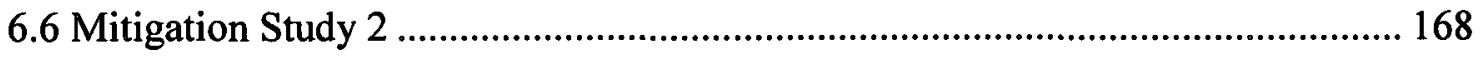

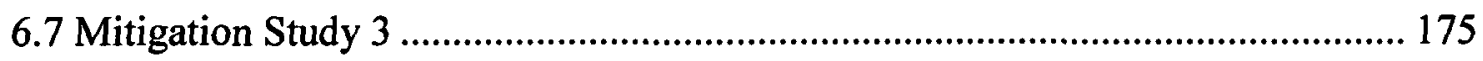

$\begin{array}{ll}\text { Chapter 7: Conclusions } & 181\end{array}$

$\begin{array}{ll}\text { References } & 185\end{array}$

Appendix A: Theory Associated with the Computational Methods Commonly Used for Analyzing the Airfoil-Vortex Interaction 198

A.1 Shear Stress Transport (SST) Model: Theory ...................................................... 198 
A.2 Subgrid Scale (SGS) Modeling: Theory .............................................................. 199

A.3 Direct Numerical Simulation (DNS)................................................................ 208

Appendix B: Viscous Blade Tip Vortices: Additional Models 212

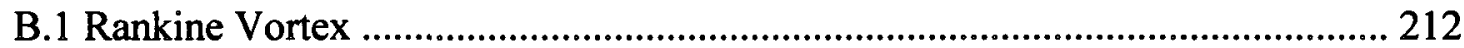

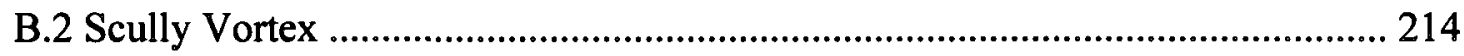

B.3 Newmans Three-Dimensional Extension of the Lamb-Oseen Vortex ............... 216

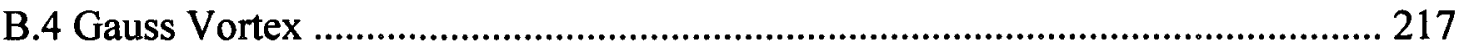

B.5 One-Dimensional Columnar Vortices ............................................................... 218

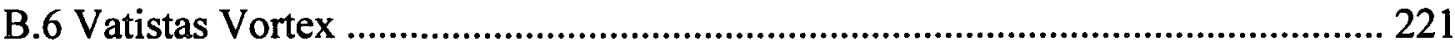

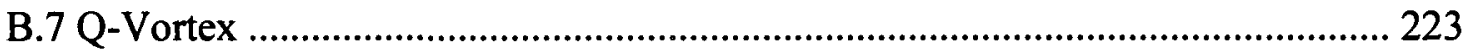

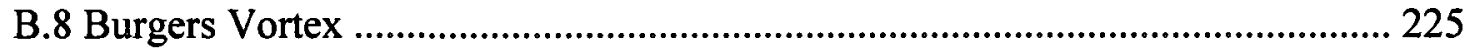

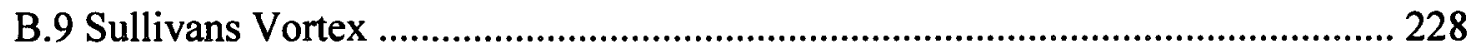

Appendix C: The Structure of Blade Tip Vortices 230 


\section{List of Tables}

Table 5.1: Node Definitions Resulting in the Compuational Domains Used for Validation 104

Table 5.2: Dimensions of the Airfoil Sections Used by Straus et al. (1990).................. 107

Table 5.3: Fluid Parameters Employed by Straus et al. (1990)........................................ 107

Table 5.4: Miss Distances of the Vortex in Straus et al. (1990) (Original) and in the

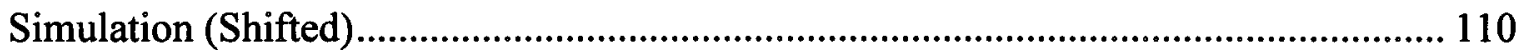

Table 5.5: Boundary Conditions Used for the Validation Analysis ............................... 115

Table 6.1: Node Definitions Resulting in the Computational Domains Used for the Mitigation Study ............................................................................................................. 146

Table 6.2: Defining Vortex Parameters for the Mitigation Studies ............................... 149

Table 6.3: Boundary Conditions Used for the Domain D and E for MS 1 and MS 2 .... 151

Table 6.4: Inlet Boundary Condition for all Domains for Mitigation Study 3 ............... 151 


\section{List of Figures}

Figure 1.1: Schematic of the beginning of a blade-vortex interaction................................ 4

Figure 2.1: Aerodynamic Phenomena Experienced by a R/C in Flight (Leishman, 2006) 8 Figure 2.2: Helicopter Noise Sources with Frequencies and Intensities of the Sources Indicated (Leishman, 2006)

Figure 2.3: Tip Vortex Creation Resulting from Bound Circulation $\Gamma$ About the Rotor

Blade (Glabel et al., 2001). 10

Figure 2.4: Blade-vortex Interactions During Descending Flight of a Typical Two-Bladed Helicopter (with 7 Blade-Vortex Interactions Indicated) (Smith and Sigl, 1995)............ 11 Figure 2.5: Incident Velocity Distribution (Normal to the Leading Edge) for a) Hovering Flight, and b) Forward Flight at an Advance Ratio of 0.3 (Leishman, 2006) 13

Figure 2.6: Flow Visualization Images of the Wake Structures in Hovering Flight (Leishman, 2006). 14

Figure 2.7: Flow Visualization of the Rotor Wake in Forward Flight Illustrating the a) Top View, and b) Side View (Leishman, 2006)

Figure 2.8: Descending Flight Paths Effect on the Interaction Between the Rotor Wake and the Rotor Blades Compared to the Wake in Level Flight (Lee and Bershader, 1994)

Figure 2.9: Various Types of Advancing Side Blade-Vortex Interactions in Rotorcraft (Sim and Schmitz, 2000) 21

Figure 2.10: BVI for a 4 Bladed Rotor (Renzoni, 1987) ................................................... 22

Figure 2.11: Path of a Vortex with Respect to the Airfoil in the Parallel Blade-Vortex

Interaction Case ( $\gamma=0$ deg) (Srinivasan, 1986)........................................................... 24

Figure 2.12: Helicopter Rotor Sound Spectrum (Johnson, 1980)....................................... 25 
Figure 2.13: Ideas of the Trace Mach Number of an Acoustic Source Point on the Rotor (Leishman, 2006) 30

Figure 2.14: Schematic for wind tunnel rotor BVI test by Caradonna and Strawn (1988)

Figure 2.15: Schematic of the Configuration Employed in the Experiment Conducted by Kitaplioglu and Caradonna (1994). 40

Figure 2.16: Acoustic Results for a Two-Bladed Rotor (Experimental and Numerical) for a) a Parallel Interaction, and b) an Oblique Interaction (Kitaplioglu and Caradonna, 1994)

Figure 2.17: Schematic for the Numerical Shock Tube Employed by Mamou et al. (2001) 51

Figure 3.1: Airfoil Approximation for the Vortex Panel Method (Anderson, 2007) ....... 68 Figure 3.2: Panels of Constant Strength Vortices Near the Trailing Edge of the Airfoil (Katz and Plotkin, 2006)

Figure 3.3: Condition Allowing the Tangential Velocity to be Determined, adapted from Anderson (2007) .................................................................................................................. 71

Figure 4.1: Depiction to Aid in Defining Circulation (Anderson, 2007) .......................... 80

Figure 4.2: Relation Between Vorticity and Circulation (Anderson, 2007) ...................... 81

Figure 4.3: Illustration of a Vortex Flow (Anderson, 2007) ............................................. 82

Figure 4.4: Typical Swirl Velocity Profile Inside a Tip Vortex (Leishman, 2006).......... 85

Figure 4.5: Measurements of the Swirl Velocity Profile of Tip Vortices at Various Wake Ages (Bhagwat and Leishman, 2002)............................................................................... 89

Figure 4.6: Viscous Core Radius Growth of Tip Vortices Trailed from Rotor Blades at Various Wake Ages (Leishman, 2006) .......................................................................... 91

Figure 4.7: Correlation of Rotor Tip Vortex Measurements in Terms of Non-Dimensional Maximum Swirl Velocities and Downstream Distance (Leishman, 2006) ....................... 96

Figure 5.1: Geometry Created for the Validation of the Computational Method............ 102

Figure 5.2: Block Definitions in ANSYS ICEM CFD ...................................................... 103 
Figure 5.3: Node Definition for the Computational Domains Used in the Validation and Verification of the Computational Method 103

Figure 5.4: NACA 0012 Centric Meshes for the Validation Analysis 105

Figure 5.5: Experimental Setup Used by Straus et al. (1990) . 106

Figure 5.6: Swirl Velocity Profile of Vortex Generated by Straus et al. (1990) 109

Figure 5.7: Original (red) and Altered (black) Straus et al. (1990) Vortex Velocity

Profiles

Figure 5.8: Comparison of the Swirl Velocity Obtained with the Lamb-Oseen Model and Straus et al.'s (1990) Altered Data 113

Figure 5.9: Boundaries for the Computational Domains 114

Figure 5.10: Lift Coefficient for Varying Vortex Location for Domains A and B ........ 118 Figure 5.11: Drag Coefficient for Varying Vortex Location for Domains A and B ...... 118 Figure 5.12: Moment Coefficient for Varying Vortex Location for Domains A and B. 119 Figure 5.13: Lift Coefficient for Varying Vortex Location for Domains A and C ........ 122 Figure 5.14: Drag Coefficient for Varying Vortex Location for Domains A and C ..... 123 Figure 5.15: Moment Coefficient for Varying Vortex Location for Domains A and C. 123 Figure 5.16: Lift Coefficient for Varying Vortex Location for Domain A Using LES and the SST Based URANS 127

Figure 5.17: Drag Coefficient for Varying Vortex Location for Domain A Using LES and the SST Based URANS 127

Figure 5.18: Moment Coefficient for Varying Vortex Location for Domain A Using LES and the SST Based URANS. 128 Figure 5.19: Velocity Field Obtained from a) an LES and b) a URANS Simulation of the Blade-Vortex Interaction . 129 Figure 5.20: Experimental Data (Circles) Obtained by Straus et al. (1990) and Discrete Vortex Method Data (Solid Line) Obtained by Renzoni (1987) 134

Figure 5.21: Lift Coefficient for Domain A Simulation (red line), Straus et al.'s (1990) Experimental Data (blue +) and Renzoni's (1987) Inviscid Discrete Vortex Method (black o) 
Figure 5.22: Moment Coefficient for Domain A Simulation (red line), Straus et al.'s (1990) Experimental Data (blue +) and Renzoni's (1987) Inviscid Discrete Vortex Method (black o)

Figure 6.1: NACA 0012 Airfoil with Straight Cavity Computational Model, referred to as Domain D

Figure 6.2: NACA 0012 Airfoil with $45^{\circ}$ Cavity Computational Model, referred to as Domain E

Figure 6.3: Block Definitions in ANSYS ICEM CFD

Figure 6.4: Node Definition for the Computational Domains Used in the Mitigation

Studies.

Figure 6.5: Computational Grid for Domain D

Figure 6.6: Computational Grid for Domain E

Figure 6.7: Boundaries for the Computational Domains D and E

Figure 6.8: Incremental Lift Coefficient Definition

Figure 6.9: Velocity Fields for the Indicated Vortex Locations for Mitigation Study 1156 Figure 6.10: Pressure Fields for the Indicated Vortex Locations for Mitigation Study 1

Figure 6.11: Lift Coefficient for Varying Vortex Location for Mitigation Study 1 (i.e.

Domains A, D and E)

Figure 6.12: Drag Coefficient for Varying Vortex Location for Mitigation Study 1 (i.e.

Domains A, D and E)

Figure 6.13: Moment Coefficient for Varying Vortex Location for Mitigation Study 1 (i.e. Domains A, D and E)

Figure 6.14: Velocity Fields for the Indicated Vortex Locations for Mitigation Study 2

Figure 6.15: Pressure Fields for the Indicated Vortex Locations for Mitigation Study 2

Figure 6.16: Lift Coefficient for Varying Vortex Location for Mitigation Study 2 (i.e.

Domains A and E) (ZM $\rightarrow$ Zero Vertical Miss Distance) 
Figure 6.17: Drag Coefficient for Varying Vortex Location for Mitigation Study 2 (i.e. Domains $\mathrm{A}$ and E) (ZM $\rightarrow$ Zero Vertical Miss Distance). 173

Figure 6.18: Moment Coefficient for Varying Vortex Location for Mitigation Study 2 (i.e. Domains A and E) (ZM $\rightarrow$ Zero Vertical Miss Distance).....

Figure 6.19: Lift Coefficient for Varying Vortex Location for Mitigation Study 3 (i.e.

Domains A and E) (ZM $\rightarrow$ Zero Vertical Miss Distance)

Figure 6.20: Drag Coefficient for Varying Vortex Location for Mitigation Study 3 (i.e.

Domains A and E) (ZM $\rightarrow$ Zero Vertical Miss Distance).

Figure 6.21: Moment Coefficient for Varying Vortex Location for Mitigation Study 3 (i.e. Domains $\mathrm{A}$ and $\mathrm{E})(\mathrm{ZM} \rightarrow$ Zero Vertical Miss Distance).

Figure A.1: Kinetic Energy Transfer in Isotropic Turbulence (Wagner et al., 2007) ... 202

Figure A.1: Depiction of the Two-Level Filtering Procedure (Wagner et al., 2007) .... 208

Figure B.1: a) Swirl Velocity Profile and, b) Static Pressure Distribution of a Rankine Vortex (Alekseenko et al., 2003)

Figure B.2: Schemes of Axisymmetric Helical Vortices (Alekseenko et al., 2003) ..... 219 Figure B.3: Two-Dimensional Swirl Velocity Profiles for Numerous Tip Vortex Models (Leishman, 2006) 223

Figure B.4: Swirl Velocity Profiles of Rankine and Burgers Vortices (Alekseenko et al., 2003) 226

Figure C. 1: Smoke Flow Visualization of a Tip Vortex Emanating from a Rotor Blade with Three Regions Indicated (1 - Laminar Region, 2 - Transitional Region, 3 Turbulent Region) (Leishman, 2006) . 230

Figure C.2: Local Richardson Number with Radial Coordinate for a Vortex Flow Obtained by Measurements at $R e_{v}=50,000$ (Leishman, 2006) 


\section{Nomenclature}

\begin{tabular}{|c|c|}
\hline $\operatorname{Re}$ & Reynolds Number [1] \\
\hline $\bar{\Gamma}$ & Circulation $\left[\mathrm{m}^{2} / \mathrm{s}\right]$ \\
\hline$\psi_{b}$ & Azimuth Angle [rad] \\
\hline$N_{b}$ & Number of Rotor Blades [1] \\
\hline$\psi_{w}$ & Wake Age [rad] \\
\hline$\mu$ & $\begin{array}{l}\text { Advance Ratio [1]; } \\
\text { Dynamic Viscosity [Pa-s] }\end{array}$ \\
\hline$\Delta$ & $\begin{array}{l}\text { Fourier Series Parameter [1]; } \\
\text { Filter Width (i.e. Length of the largest Subgrid Scale Eddy) [m] }\end{array}$ \\
\hline$r$ & Radial Coordinate Along the Rotor Blade [m] \\
\hline$R$ & $\begin{array}{l}\text { Rotor Disk Radius [m]; } \\
\text { Vortex Length Scale [m] }\end{array}$ \\
\hline$\left(x_{v}, y_{v}\right)$ & Instantaneous Location of Vortex Relative to Airfoils Leading Edge [m] \\
\hline$\Omega$ & $\begin{array}{l}\text { Rotor Disk Rotational Rate }[\mathrm{rad} / \mathrm{s}] \\
\text { Flow Volume }\left[\mathrm{m}^{3}\right] \\
\text { Angular Velocity of the Solid Body Type Rotation of the Fluid within } \\
\text { Vortex Core }[\mathrm{rad} / \mathrm{s}]\end{array}$ \\
\hline$M$ & $\begin{array}{l}\text { Mach Number [1]; } \\
\text { Time Step Number [1] }\end{array}$ \\
\hline$M_{t r}$ & Trace Mach Number [1] \\
\hline$r_{b}$ & Radial Position of a Blade-Vortex Interaction Along a Rotor Blade [m] \\
\hline$U$ & Characteristic Velocity $[\mathrm{m} / \mathrm{s}]$ \\
\hline
\end{tabular}




\begin{tabular}{|c|c|}
\hline$p$ & $\begin{array}{l}\text { Static Pressure }[\mathrm{Pa}] ; \\
\text { Sound Pressure }[\mathrm{Pa}]\end{array}$ \\
\hline$\rho$ & Density $\left[\mathrm{kg} / \mathrm{m}^{3}\right]$ \\
\hline$(u, v, w)$ & Cartesian Components of Velocity [m/s] \\
\hline$\left(v_{r}, v_{\theta}, v_{z}\right)$ & Cylindrical Components of Velocity [m/s] \\
\hline$a$ & $\begin{array}{l}\text { Speed of Sound }[\mathrm{m} / \mathrm{s}] \\
\text { Length Scale for Vortex Diffusion }[\mathrm{m}]\end{array}$ \\
\hline$\gamma$ & $\begin{array}{l}\text { Angle Between Blade and Vortex Axes [rad]; } \\
\text { Circulation per Unit Length [m/s] }\end{array}$ \\
\hline$\beta$ & $\begin{array}{l}\text { Ray Cone Semi-Vertical Angle [rad]; } \\
\text { Angle Between a Panels Normal and the Freestream Direction [rad] }\end{array}$ \\
\hline$A$ & $\begin{array}{l}\text { Rotor Disk Area }\left[\mathrm{m}^{2}\right] \text {; } \\
\text { Constant Related to Lift and Drag on a Lift Generating Surface [1] }\end{array}$ \\
\hline$\overline{A_{b}}$ & Rotor Blade Planform Area $\left[\mathrm{m}^{2}\right]$ \\
\hline$T$ & Rotor Thrust [N] \\
\hline$W_{b}$ & Rotor Power [W] \\
\hline$\varepsilon$ & $\begin{array}{l}\text { Turbulence Dissipation Rate [W/kg]; } \\
\text { Typical Vortex Size [m] }\end{array}$ \\
\hline$v$ & Kinematic Viscosity $\left[\mathrm{m}^{2} / \mathrm{s}\right]$ \\
\hline$v_{T}$ & Turbulent Viscosity $\left[\mathrm{m}^{2} / \mathrm{s}\right]$ \\
\hline$v_{e}$ & Eddy Viscosity $\left[\mathrm{m}^{2} / \mathrm{s}\right]$ \\
\hline$\omega$ & $\begin{array}{l}\text { Turbulence Frequency }[\mathrm{rad} / \mathrm{s}] \\
\text { Vorticity }\left[\mathrm{m}^{2} / \mathrm{s}\right]\end{array}$ \\
\hline$\tau_{i j}$ & $\begin{array}{l}\text { Stress Tensor }[\mathrm{Pa}] \\
\text { Reynolds Stress Tensor }[\mathrm{Pa}]\end{array}$ \\
\hline$k$ & Turbulence Kinetic Energy $\left[\mathrm{m}^{2} / \mathrm{s}^{2}\right]$ \\
\hline$\overline{S_{i j}}$ & Strain Rate Tensor $\left[\mathrm{s}^{-1}\right]$ \\
\hline$\delta_{i j}$ & Kronecker Delta [1] \\
\hline
\end{tabular}




\begin{tabular}{|c|c|}
\hline$\phi$ & $\begin{array}{l}\text { Blending Parameter in SST model [1]; } \\
\text { Velocity Potential }\left[\mathrm{s}^{-1}\right]\end{array}$ \\
\hline$F$ & Weighting Functions for SST Blending Process [1] \\
\hline $\arg$ & Argument Determining Weights in Weighting Functions for SST [rad] \\
\hline$y$ & Vertical Distance of the Node Closest to the Surface Wall [m] \\
\hline $\mathrm{CD}_{k \omega}$ & Cross-Diffusion Term [1] \\
\hline$a_{1}$ & Eddy Viscosity Constant [1] \\
\hline$G$ & Averaging Kernel [1] \\
\hline$U_{L}$ & Velocity Scale of the Largest Eddies $[\mathrm{m} / \mathrm{s}]$ \\
\hline$L$ & $\begin{array}{l}\text { Integral Length Scale of a Turbulent Flow [m]; } \\
\text { Characteristic Length [m] }\end{array}$ \\
\hline$U_{S G S}$ & Velocity Scale of the Smallest Eddies $[\mathrm{m} / \mathrm{s}]$ \\
\hline$C_{s}$ & Smagorinsky Constant [1] \\
\hline$K_{o}$ & Constant [1] \\
\hline$y^{+}$ & Non-Dimensional Distance from Surface (Boundary Layer Parameter) [1] \\
\hline$q$ & $\begin{array}{l}\text { Transport Energy }\left[\mathrm{m}^{2} / \mathrm{s}^{2}\right] \\
\text { Shape Factor }[1]\end{array}$ \\
\hline$C_{s}^{*}$ & Dynamic Smagorinsky Constant [1] \\
\hline$\eta$ & Kolmogorov Length Scale [m] \\
\hline$\tau$ & Kolmogorov Time Scale [s] \\
\hline$L_{i}$ & Integral Length Scale [m] \\
\hline$R_{i j}$ & Correlation Tensor [1] \\
\hline$(\Delta x, \Delta y, \Delta z)$ & $\begin{array}{l}\text { Grid Spacing in }(x, y, z) \text { Directions [1]; } \\
\text { Grid Definition Parameters [1] }\end{array}$ \\
\hline Cor $C F L$ & Courant- Friedrichs-Lewy Number [1] \\
\hline$N$ & Number of Panels [1] \\
\hline$\theta_{i j}$ & Angle of Vector Connecting Two Panels Midpoints [rad] \\
\hline$n$ & Normal Direction [1]; \\
\hline
\end{tabular}




\begin{tabular}{|c|c|}
\hline & Integer [1] \\
\hline$s$ & Panel Length [m] \\
\hline$L^{\prime}$ & Lift per Unit Span $[\mathrm{N} / \mathrm{m}]$ \\
\hline$D^{\prime}$ & Drag per Unit Span $[\mathrm{N} / \mathrm{m}]$ \\
\hline$M^{\prime}$ & Moment per Unit Span [N] \\
\hline$\dot{\theta}$ & Angular Velocity of a Fluid Element [rad/s] \\
\hline$\vec{v}$ & Velocity Vector $[\mathrm{m} / \mathrm{s}]$ \\
\hline$r_{c}$ & Viscous Core Radius of a Tip Vortex [m] \\
\hline$\alpha$ & Lamb-Oseen Model Parameter [1] \\
\hline$(x, y, z)$ & Coordinates in a Cartesian Coordinate System [m, m, m] \\
\hline$(r, \theta, z)$ & Coordinates in a Cylindrical Coordinate System [m, rad, rad] \\
\hline$\psi$ & Stream Function $\left[\mathrm{s}^{-1}\right]$ \\
\hline$\Delta p$ & Pressure Drop [Pa] \\
\hline$l$ & Pitch of a Helical Vortex [m] \\
\hline$t$ & Time $[\mathbf{s}]$ \\
\hline$R e_{v}$ & Vortex Reynolds Number [1] \\
\hline$\delta$ & Turbulent Viscosity Coefficient [1] \\
\hline$c$ & Airfoils Chord Length [m] \\
\hline $\bar{d}$ & Non-Dimensional Vortex Position [1] \\
\hline$R i$ & Gradient Richardson Number [1] \\
\hline$\Delta(x y)$ & Node Definition of Diagonal Segments (i.e. O-grid) [1] \\
\hline$C_{l}$ & Lift Coefficient [1] \\
\hline$C_{d}$ & Drag Coefficient [1] \\
\hline$C_{m}$ & Pitching Moment Coefficient About the Airfoils Leading Edge [1] \\
\hline$C_{p}$ & Pressure Coefficient [1] \\
\hline$\Delta C_{l}$ & Incremental Lift Coefficient [1] \\
\hline
\end{tabular}




\section{Subscripts}

\begin{tabular}{c|l}
$\infty$ & Freestream Property \\
\hline$o$ & Vortex Axis Property \\
\hline$(i, j, k)$ & Representative Indices of the Coordinate System \\
\hline$m$ & Mean Vortex Property \\
\hline$t i p$ & Parameter at the Blade Tip
\end{tabular}

\section{Accents}

\begin{tabular}{c|l}
- & $\begin{array}{l}\text { Non-Dimensional Parameter, } \\
\text { Average Component of a Parameter (i.e. Velocity), } \\
\text { Filtered Parameter }\end{array}$ \\
\hline$\sim$ & Test Filtered Parameter \\
\hline, & Fluctuating Component of a Parameter (i.e. Velocity)
\end{tabular}

\section{Abbreviations}

\begin{tabular}{c|l} 
BVI & Blade-Vortex Interaction \\
\hline AVI & Airfoil-Vortex Interaction \\
\hline CFD & Computational Fluid Dynamics \\
\hline LES & Large Eddy Simulation \\
\hline RANS & Reynolds-Averaged Navier-Stokes \\
\hline URANS & Unsteady Reynolds-Averaged Navier-Stokes \\
\hline SGS & Subgrid Scale \\
\hline R/C & Rotorcraft (or Helicopter) \\
\hline A/C & Aircraft \\
\hline AOA & Angle of Attack \\
\hline LE & Leading Edge \\
\hline TE & Trailing Edge
\end{tabular}




\section{Chapter 1: Introduction and Motivation}

This thesis presents a comprehensive study pertaining to the aerodynamic investigation of a two-dimensional (2D) blade-vortex interaction (BVI), which is also referred to as an airfoil-vortex interaction (AVI).

The current chapter provides a brief description of the problem, followed by the motivation which inspired the current research. The methodology employed to solve the problem along with the major objectives of the current work shall then be outlined. A literature review concerning BVI and some background pertaining to the problem at hand shall then be provided (Chapter 2). The numerical methods employed in Computational Fluid Dynamics (CFD) which were used to resolve the problem shall then be addressed (Chapter 3). Some theory and a literature review related to blade tip vortices are provided in Chapter 4. The theory presented in Chapter 4 is pertinent for any AVI investigation. Chapter 5 validates and verifies the use of Large Eddy Simulation (LES) to solve the AVI problem. The use of LES for AVI investigations is both validated and verified through a comparison of experimental results of Straus et al. (1990). The results obtained from a discrete vortex method presented by Renzoni (1987) were compared with the results obtained for LES with the same inflow and vortex parameters employed previously. Chapter 6 presents a study that attempts to mitigate the interaction between the tip vortex 
and the blade. This is done by placing two different cavities in the blades, one slanted and one straight to quantitatively view the change of the aerodynamics of the blade-vortex interaction through the application of these cavities. Other studies based on the mitigation study are performed in Chapter 6 by varying the miss distance of the vortex (defined in Figure 1.1) as well as the freestream velocity using the unaltered geometry as well as the cavity which provides the most beneficial results related to the aerodynamics of the BVI phenomenon. Finally, Chapter 7 presents some concluding remarks.

\subsection{Blade-Vortex Interaction}

Blade-vortex interaction (BVI) is a rotorcraft specific phenomenon which occurs as a result of the aerodynamic interaction of rotor blades of a helicopter with vortices shed by preceding rotor blades. This phenomenon is inescapable and is a result of the confined airspace available for the lifting bodies of a helicopter (i.e. the rotor blades). In rotorcraft, one of the main sources of noise and vibrations is BVI as a result of the complex unsteady flow features occurring during the interaction. During ascent, descent, hover, and lateral flight (forward, reverse, or sideways) a blade travels almost directly through the wake of all the preceding blades and possibly even through its own wake from a previous rotation. This produces a complex and undesirable aerodynamic situation which is unique to helicopters, in which the blades encounter numerous fluid events that are extremely difficult to predict. These complex interactions are most prominent during descent and rotorcraft maneuvers which result in the aforementioned noise and production of vibrations. 
On any lifting body of finite length, the pressure differential between the upper and lower surfaces an undesirable side-effect at the edge of the lifting body. Namely, at the edge of the lifting body the high pressure fluid attempts to reach equilibrium by means of seeping around the edge to the upper surface, i.e. the location of the lower pressure fluid. The curling motion around the edge of the lifting body results in localized vorticity, in the form of a vortex line as a result of the relative motion between the lifting body and the fluid.

When a vortex is shed from the tip of a rotor blade, it is convected downstream as a result of the rotating frame and the confined airspace (i.e. the wakes confinement to the rotor disk plane), thus resulting in the intersection of the vortex and the path of the advancing rotor blade. A vortex whose axis is parallel (or nearly parallel) to the spanwise axis causes the most significant interactions of the blade due to strong chordwise temporal pressure variations during the interaction. Figure 1.1 depicts schematically, a vortex at an upstream location of the airfoil's leading edge of as it advects towards the airfoil at the onset of BVI. Figure 1.1 also illustrates the vortex at a certain vertical distance between the chord line of the blade and its core (referred to as the miss distance) as a result of the downwash effect resulting from the generation of lift.

This problem is commonly observed in fixed wing aircraft as well; however, small vertical wing sections called winglets have been designed to be placed on the wing tip in order to reduce the induced drag. Winglets reduce induced drag at the wing tip by effectively increasing the aspect ratio of the wing with no additional wingspan. This issue does not consider the effect of the shed vortices downstream of the aircraft, which is 
responsible for BVI. As a result of the rotational nature of the helicopter along with the additional lateral motion, winglets are difficult to implement. Therefore, winglets are not as easily implemented in rotorcraft as in fixed wing aircraft.

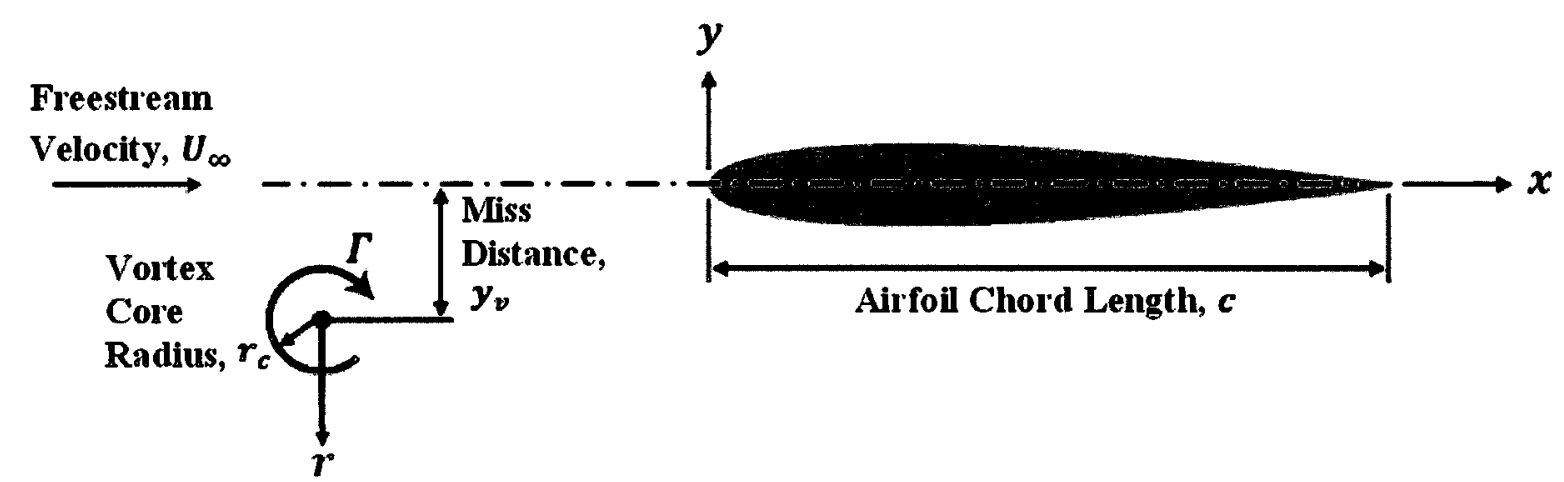

Figure 1.1: Schematic of the beginning of a blade-vortex interaction

As a result of the lack of a "quick fix," numerical simulation of BVI has been of interest to the Computational Fluid Dynamics (CFD) community for many years. However, the accurate numerical prediction of BVI remains difficult. One of the main issues is the inherent numerical dissipation of CFD models, which may severely affect the persistence of the vortex characteristics. Accurate prediction of BVI airloads using the unsteady Reynolds-Averaged Navier-Stokes (URANS) relations is very challenging due to the complexity of the flow dynamics particularly with respect to the development of the boundary layer on the suction side of the airfoil as well as flow separation. The use of RANS methods rely on turbulence models to represent all the relevant scales of turbulence. RANS methods employ the mean flow properties to predict the acoustic noise. Since noise generation is a multi-scale problem which involves a wide range of length and time scales, the use of RANS presents limitations on the prediction of the noise generated by the BVI phenomenon. Although RANS methods are useful for 
predicting the aerodynamic coefficients in a reasonable manner, they are neither suitable nor reliable for accurate noise prediction.

Recent improvements in the processing speed of computers have increased the feasibility of applying Direct Numerical Simulations (DNS) or Large Eddy Simulations (LES) to complex turbulent flows. Nevertheless, DNS is limited to low Reynolds number flows with relatively simple geometries due to the wide range of length and time scales present in turbulent flows. Since the number of grid points required for DNS is known to be proportional to $R e^{9 / 4}$, DNS of high Reynolds number flows require high grid resolution far beyond the capability of the most powerful computers. In order to overcome the issues relating to grid requirements, turbulence must be modeled in order to obtain results of practical interest using simulations. LES is a promising alternative to DNS for simulations involving high Reynolds number flows as it has a lower computational cost. In LES, the large scales are directly solved while the small scales are modeled. Note that noise generation is an unsteady process. LES is presently the most affordable computational tool to be used, as it is currently the only means (besides DNS) to obtain a time-accurate unsteady solution. Thus, the present analysis is based on an LES approach.

\subsection{Motivation}

As of late, helicopter use has substantially increased in both military and civilian sectors. This increased usage has motivated extensive research to more accurately estimate the blade airloads to improve the performance and stability of the rotorcraft as well as to reduce its generated noise. Moreover, reducing rotorcraft noise is perhaps the 
strongest current motivation for researchers with a tremendous weight placed on increasing the understanding of the flow physics present in a rotor. But, progress has been made in BVI noise prediction. Recently, the growing public demand for more stringent noise regulations has led the rotorcraft industry to develop procedures to reduce BVI noise in the direct vicinity of urban areas. In addition to the adverse public opinion of acoustic noise generated by a rotorcraft, the BVI induced vibratory loads increase the pilot's workload, reduce the components fatigue life, and increase maintenance costs. Therefore, the issues of vibration and noise production require the designer to focus primarily on the reduction of these features to the lowest possible levels. The motivation of the present research is based on the need for a better understanding of a BVI event as well as an attempt to minimize the generation of unsteady airloads during this interaction.

\subsection{Research Objectives and Approach}

The main objectives of the current work are to validate and verify the use of LES for investigations related to the aerodynamics of AVI as well as to mitigate the interaction. Based on the geometry which presents the most benefits related to the aerodynamics of the problem, parametric studies are presented to ensure the AVIs effect is mitigated for numerous flight conditions.

In order to investigate the aforementioned objectives LES shall be employed. Also, careful attention has been used with respect to appropriately modeling the blade tip vortex and the Lamb-Oseen model (as outlined in Sub-Section 4.3.1) shall be employed. 


\section{Chapter 2: Blade-Vortex Interactions}

The intensity and directivity of noise generated by a rotorcraft $(\mathrm{R} / \mathrm{C})$ is of considerable importance in both civilian and military applications. The present certification and community noise constraints which apply to helicopters are quite strict, especially during take-off and landing (Lowson, 1992). In military applications there is also a demand to abate noise in order to reduce the detectability of the R/C. Despite being the quietest vertical take-off and landing (VTOL) aircraft, the helicopters noise level is still sufficient to compromise its use, unless specific attention is paid to low noise during the design process. As the restrictions related to aircraft noise increase, the noise produced by the rotor becomes an increasingly important factor in $\mathrm{R} / \mathrm{C}$ design.

Acoustic noise results from the operation of a propulsive, aerodynamic system. For example, when a fixed wing aircraft generates noise from the airframe and the engine, the sound's intensity increases with both thrust and aircraft speed. Additionally, $\mathrm{R} / \mathrm{Cs}$ are equipped with a rotor system which is the main source of thrust, control and the associated noise. Fixed wing aircrafts have a relatively uniform lift loading over the entire span. The wing wake and tip vortices generated by the pressure difference across the finite lifting body are trailed downstream of the aircraft. The flow field for a rotor for an $\mathrm{R} / \mathrm{C}$ in forward flight, illustrated in Figure 2.1, is much more complex than for a wing 
fixed wing aircraft since individual wakes are trailed from each blade (Leishman, 2006) regardless of the R/Cs operating condition.

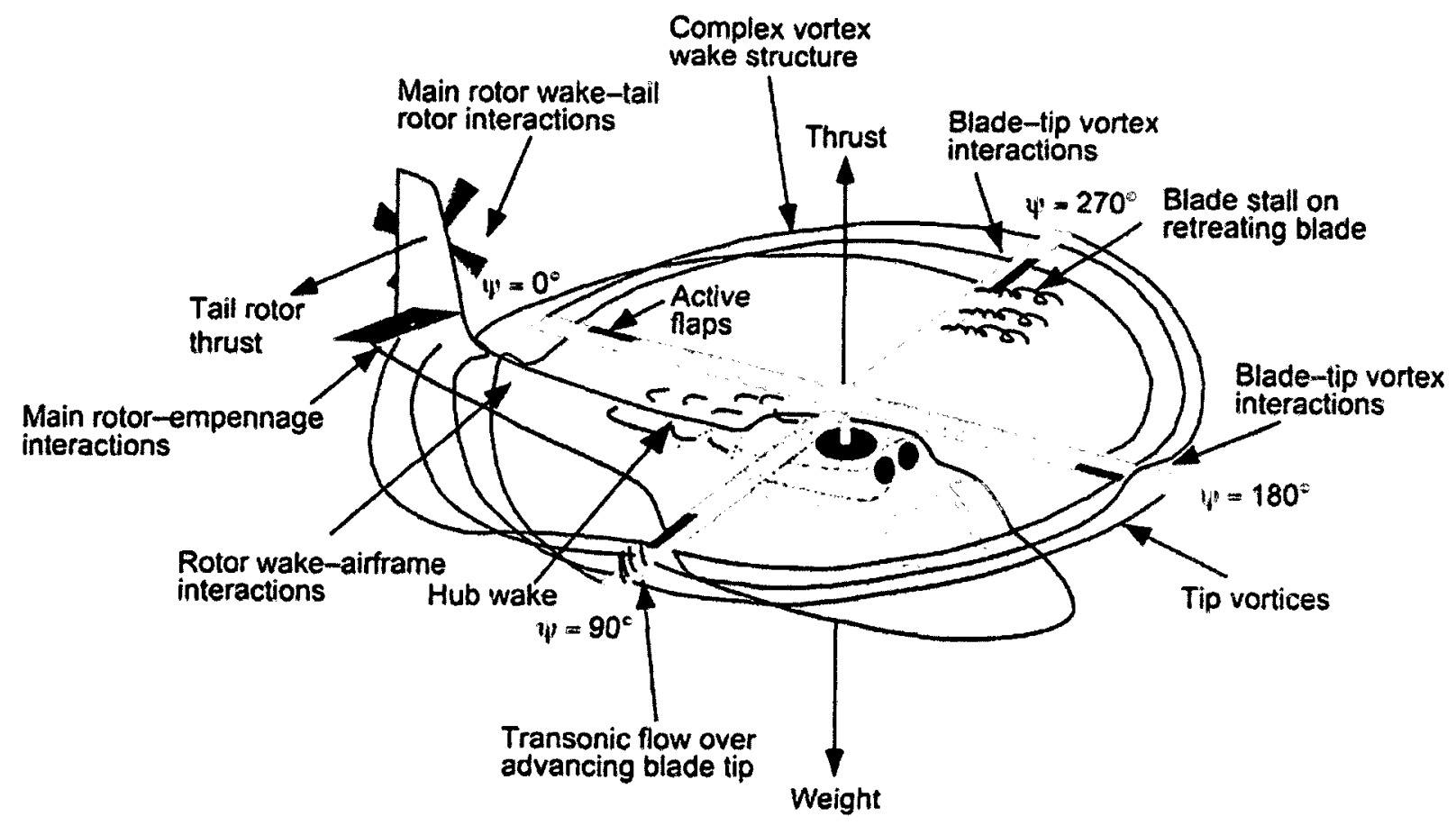

Figure 2.1: Aerodynamic Phenomena Experienced by a R/C in Flight (Leishman, 2006)

On the advancing side of the disk, numerous aerodynamic phenomena occur, as is illustrated in Figure 2.1, the main feature of which include supercritical (or compressible) flow and blade-vortex interaction (BVI). The main aerodynamic features on the retreating side of the disk are blade (or dynamic) stall and a region of reverse flow near the rotor hub.

During R/C flight, the vortices formed at the blade tip induce sharp, periodic aerodynamic disturbances on the next advancing blade. These disturbances are responsible for the generation of the highly impulsive (and obtrusive) noise associated with BVI, as depicted in Figure 2.1. There are two types of this highly impulsive noise, 
which is referred to as discrete frequency noise: these include BVI and high-speed impulsive noise (HSI). Discrete frequency noise generally occurs at low frequency and high amplitude, as is illustrated in Figure 2.2, which also shows numerous other continuous spectrum noise sources during $\mathrm{R} / \mathrm{C}$ flight.

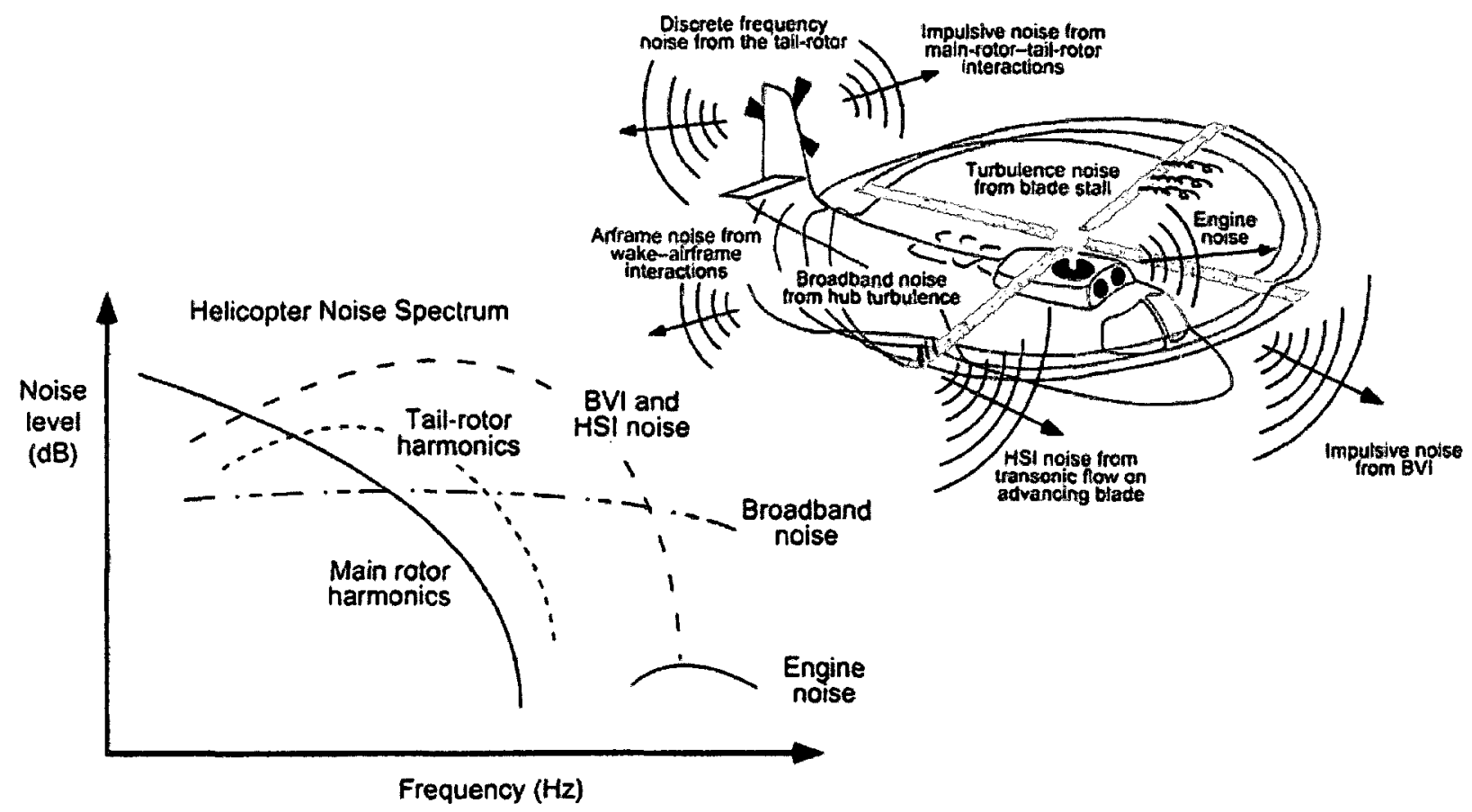

Figure 2.2: Helicopter Noise Sources with Frequencies and Intensities of the Sources Indicated (Leishman, 2006)

Regardless of flight condition, rotary wing aircraft produce a continuous vortex sheet that rolls up to form two single coherent trailing vortex lines at the hub and the tip edges of the blade as is illustrated in Figure 2.3. 


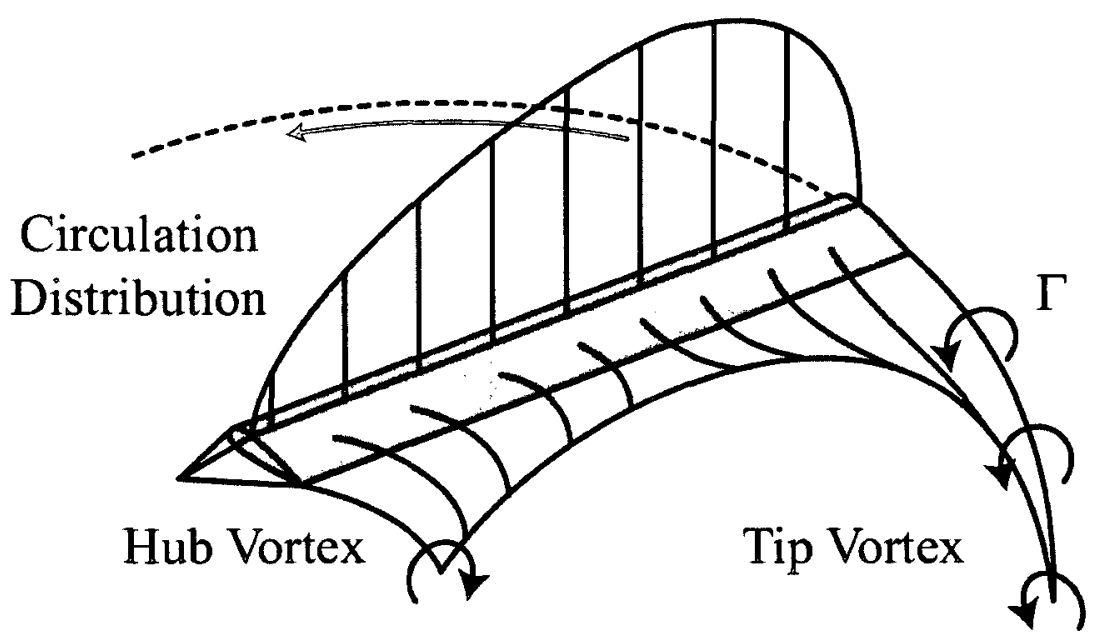

Figure 2.3: Tip Vortex Creation Resulting from Bound Circulation $\Gamma$ About the Rotor Blade (Glabel et al., 2001)

The hub vortex is quickly pushed beneath the rotor as a result of the strong downwash present near the hub, thereby becoming relatively well removed from the blade path. Therefore this portion of the vortex sheet does not have a significant impact on the BVI. On the other hand, the tip vortex remains present within the aerodynamic area of influence of the main rotor. It is characterized by a continuous line vortex traced out by the blade tip as the rotor travels along its circular path. As a result, the tip vortex may interact with the remaining blades over different stages of the rotor revolution depending on the R/Cs movement. This is shown in both the plan and side view in Figure 2.4 for a two-bladed rotor configuration.

As a consequence of the pressure differential which is characteristic of any lift generating body, the net flow velocity at the rotor plane, or wake, is composed of velocities induced by the tip vortices. Consequently, the blades' airloads and rotor performance are based on both the strength and locations of the tip vortices (Leishman, 
2006). The locations of interaction between the tip vortices and the rotor blades, i.e. BVI, are indicated in Figure 2.4 as number 1 through 7.

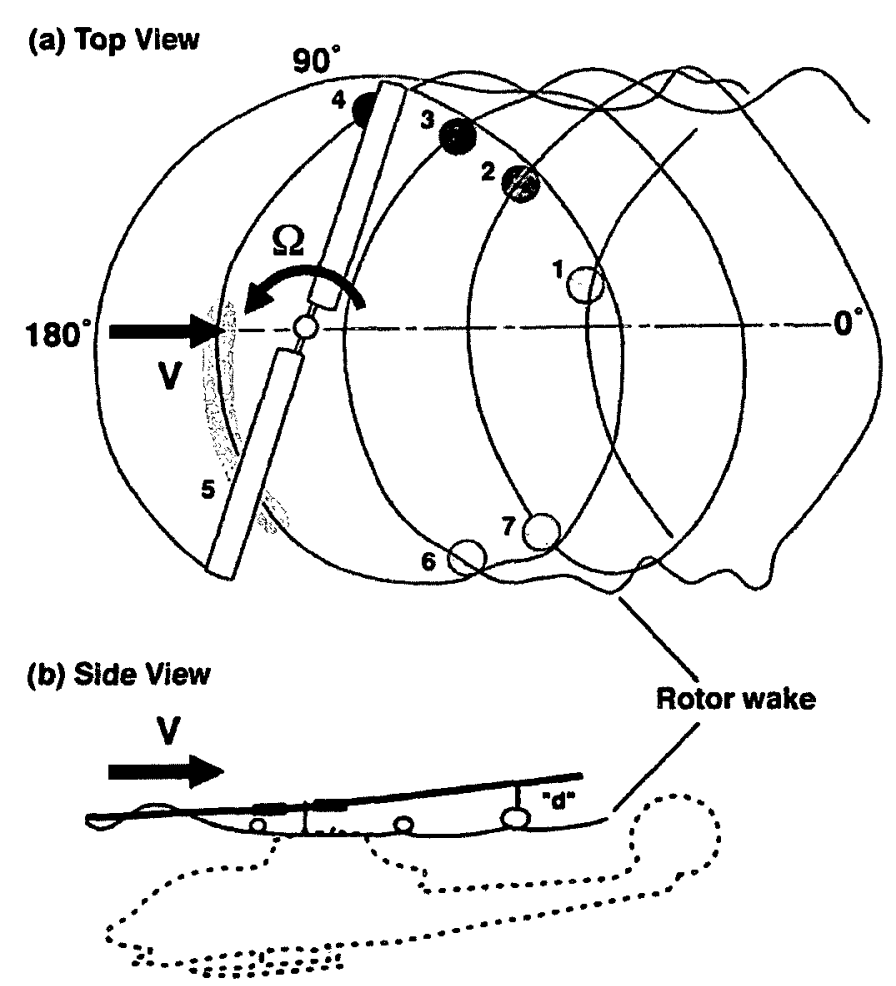

Figure 2.4: Blade-vortex Interactions During Descending Flight of a Typical Two-Bladed Helicopter (with 7 Blade-Vortex Interactions Indicated) (Smith and Sigl, 1995)

Rotor wakes are dominated by the strong vortices trailed from the tips of each blade. The nature of the rotor wakes in terms of geometry, strength and aerodynamic effects on the blades depends on the flight condition of the R/C. Recent advances in measurement techniques allow for more satisfactory wake measurements to be performed; however, the formation and evolution of the tip vortices and rotor wakes requires further research. Leishman and Bagai (1998) provide an overview of the key physical features characteristic of rotor wakes and of the challenges involved with their measurement. Landgrebe (1998) and McCorskey (1995) review the capabilities for 
modeling rotor wakes. Recent developments in modeling rotor wakes have shown that close BVI encounters (i.e. interactions with small vertical miss distances) occur for low and moderate forward flight speeds (Egolf and Landgrebe, 1983). Because the movement of the tip vortices has been found to be above and then down through the rotor disk, many such BVI encounters occur at low advance ratios. This supports the inevitability of BVIs in low speed forward flight as is illustrated in Figure 2.4b. In these flight conditions a clockwise rotating (negative) vortex is generated by an advancing blade, followed by a counter-clockwise rotating (positive) vortex inboard of the tip vortex as is depicted in Figure 2.3. The inboard vortex, however, due to the gradual radial load variation, does not roll up completely prior to encountering the following blades (Hooper, 1983).

The wake and resultant tip vortices also vary with flight condition. For instance, in hover, the rotor blades encounter a symmetric velocity profile, which is characterized by a maximum velocity on the advancing side of the disk at the tip of blade and an equal and opposite velocity at the tips of the retreating blades, as is shown in Figure 2.5a.

In hover, the tip vortices follow helical trajectories below the rotor. The wake in hover is radially axisymmetric and consists of two distinct features, the tip vortices and shear layers which may be seen in Figure 2.6. The shear layers (as seen on the right hand side of the circular tip vortices in Figure 2.6) are formed by merging boundary layers from the upper and lower surfaces of the blades, which contain both negative and positive vorticity respectively. This shear layer found within the rotor wake in hover is often referred to as vortex sheets. 
In forward flight, however, the rotor blades encounter an asymmetric velocity field, as can be seen in Figure 2.5b. In forward flight, the rotor wake is skewed behind the rotor by the oncoming flow. This forms a series of complex interlocking epicycloidal vortex trajectories as illustrated in Figure 2.7. The wake in forward flight is dominated by the blade tip vortices, however since the freestream component of velocity is present at the rotor plane, the wake is not only convected below the rotor, but behind the $\mathrm{R} / \mathrm{C}$ as well resulting in an asymmetric velocity distribution.

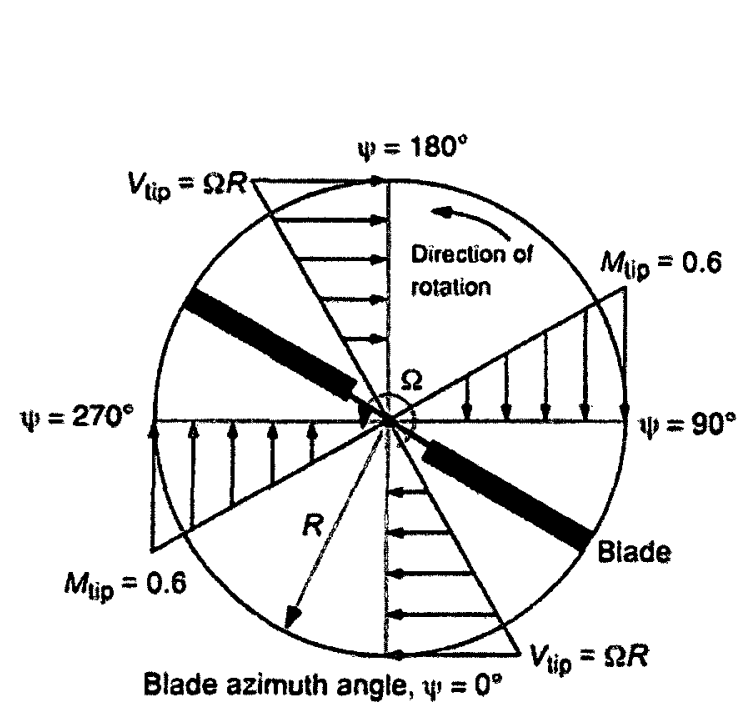

(a) Hovering flight

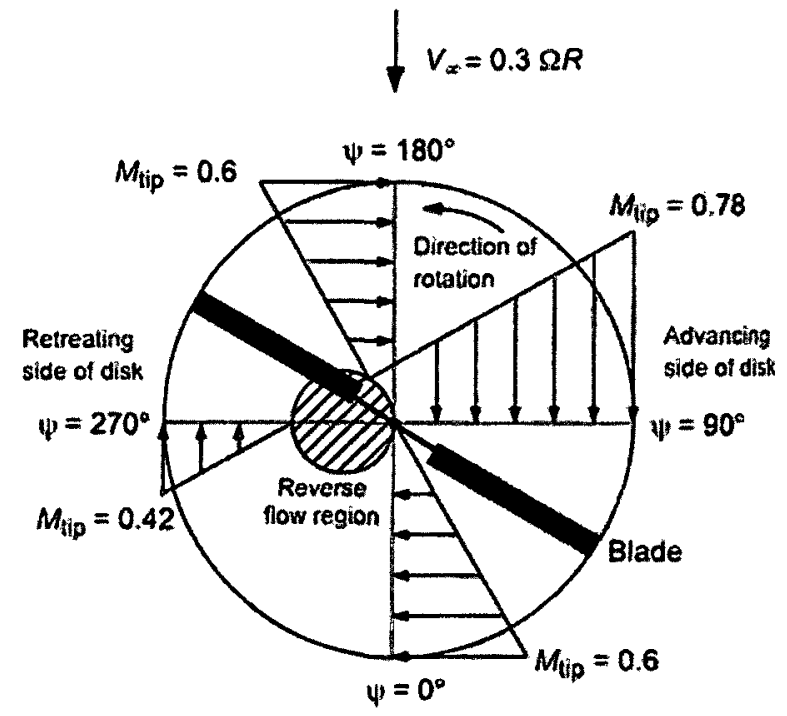

(b) Forward flight, $\mu=0.3$

Figure 2.5: Incident Velocity Distribution (Normal to the Leading Edge) for a) Hovering Flight, and b) Forward Flight at an Advance Ratio of 0.3 (Leishman, 2006) 


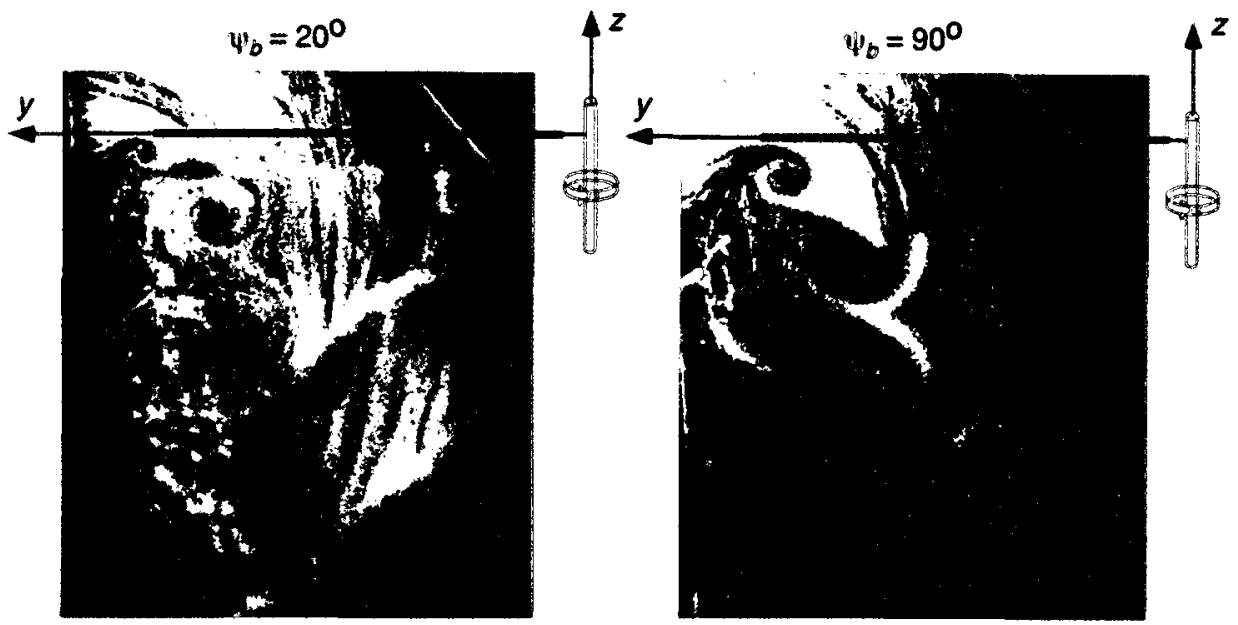

Figure 2.6: Flow Visualization Images of the Wake Structures in Hovering Flight (Leishman, 2006)

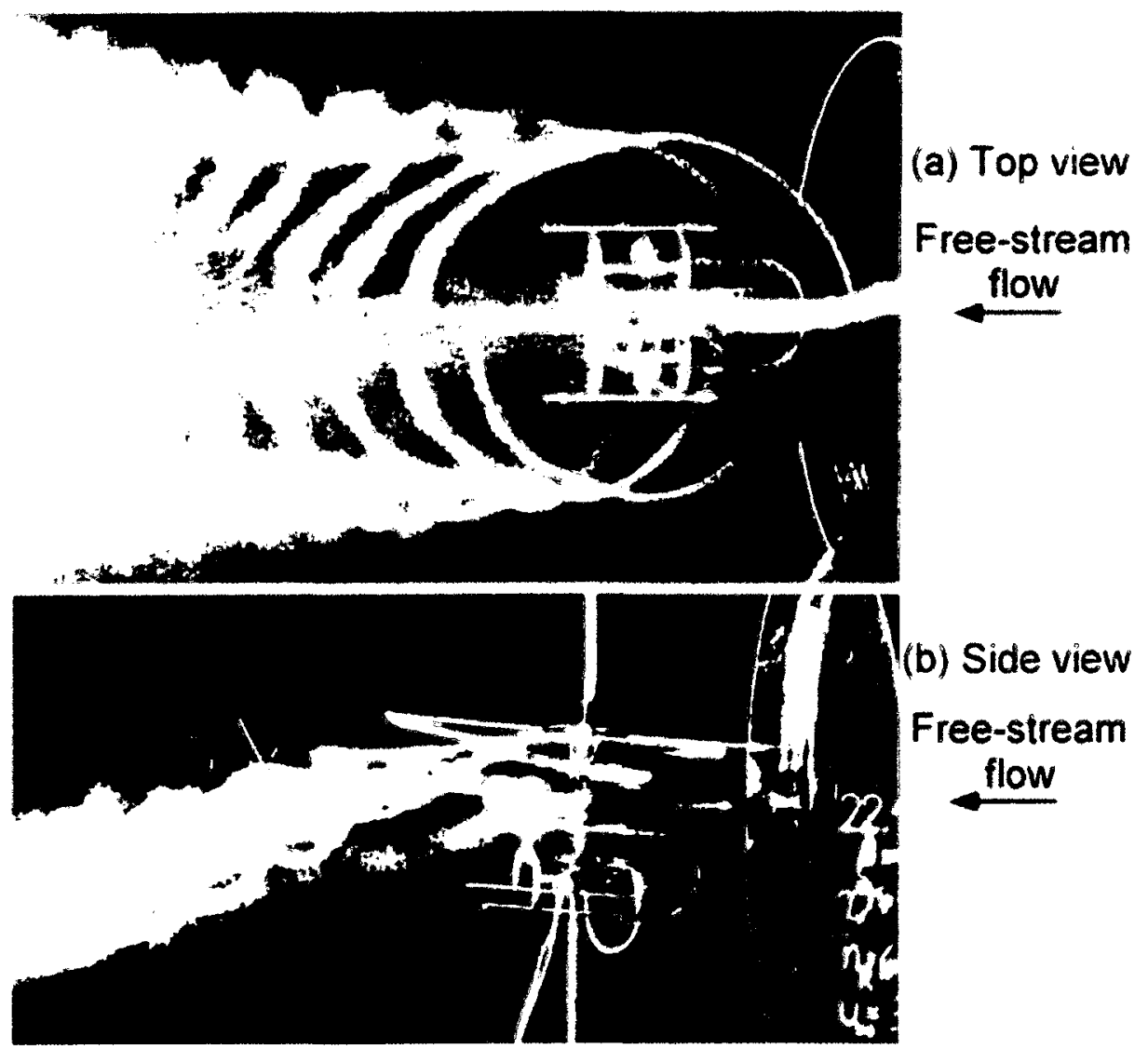

Figure 2.7: Flow Visualization of the Rotor Wake in Forward Flight Illustrating the a) Top View, and b) Side View (Leishman, 2006) 
An R/C in forward flight has the potential for the blade tip vortices to remain close to the rotor or following blades for several rotor revolutions. Therefore, on account of the low disk loading and generally low average flow velocities through the rotor disk, these vortices remain close enough to the rotor disk to produce a strongly three dimensional induced velocity field. As the following blades encounter the induced velocity field, fluctuating airloads are produced on each rotor blade. These time-varying airloads may be a high source of rotor vibrations, strongly focused obtrusive noise, and the reduction of the performance of the rotor disk as well as helicopter instability. As a result, interest in the interactions of rotor blades with the concentrated tip vortices has increased in recent years. Srinivasan and McCorskey (1987) have showed that the effect of the vortex trajectory distortion appears to be of secondary importance to the overall airloads. This is not the case for transonic speeds however as the vortex may pass close to a shockwave which may alter its characteristics and path.

It has been shown that BVIs are responsible for the high impulsive noise associated with R/C during powered descending flight and maneuvers (Charles. 1975; and Pegg, 1979). Under these operating conditions, low rotor disk flow results in the concentrated tip vortices being blown back into the rotor disk, thus causing numerous BVIs. Since the vortex is more likely to interact with the rotor as it is drawn closer to the rotor disk, BVI is most prominent during low speed descent. The noise produced during BVI events in powered descent is often radiated downward and it is dominant over other R/C noise sources. The most significant effects of BVI, up to moderate advance ratios as well as maneuvering $\mathrm{R} / \mathrm{C}$ flight, occur in descent and forward flight because during 
descent, forward flight and maneuvers, the tip vortices and the rotor blades have closely intersecting paths as is shown in Figure 2.8.
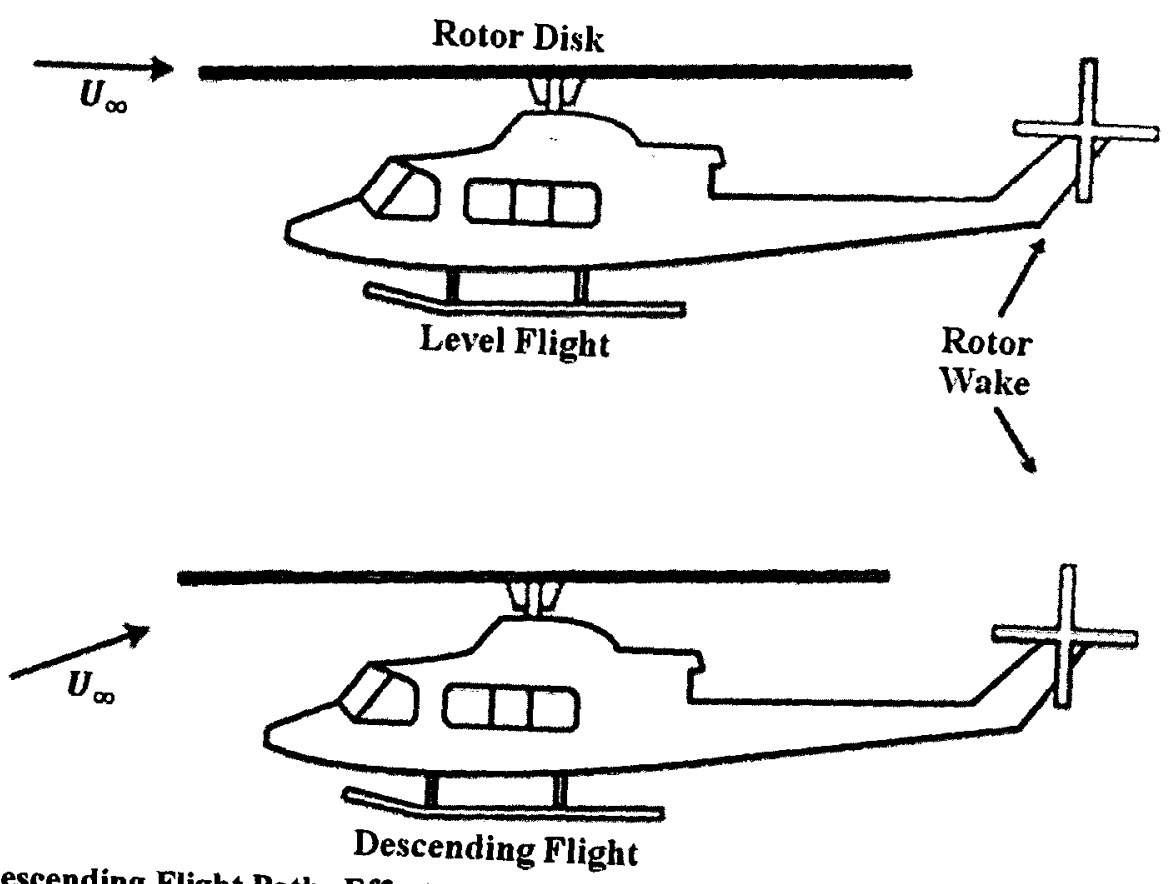

Figure 2.8: Descending Flight Paths Effect on the Flight

Blades Compared to the Wake in Level Flight Between the Rotor Wake and the Rotor in Level Flight (Lee and Bershader, 1994)

In forward level flight, the tip vortices flow down and out of the rotor's path on account of the downwash from the R/Cs main rotor (see Figure 2.8). Contrarily, in descending forward flight the downwash is counteracted by the freestream. This results in the tip vortices passing directly through the main rotors path as shown in Figure 2.8, which causes seven significant BVIs on both the advancing and retreating sides of the rotor disk as shown in Figure 2.4. On the advancing side of the rotor disk, BVIs occur between 20 and 70 degrees azimuth and between 300 and 350 degrees azimuth in the retreating quadrant BVIs (Egolf and Landgrebe, 1983; Martin et al., 1986). The seven aforementioned locations of the most significant BVIs through one complete revolution of the main rotor in descending flight for both the advancing and retreating sides of the 
rotor disk are shown in Figure 2.4. In Figure 2.4, locations 1 through 4 represent the interactions on the advancing side and locations 5 through 7 are the interactions found on the retreating side. The retreating side interactions are less intense than the advancing side interactions, as the advancing side interactions occur at a much higher Mach number due to the additive nature of the freestream and radial blade velocities (see Figure 2.5). As a result the advancing side interactions result in more intense far-field noise production (Martin et al., 1986; Yu, 2000) and the retreating side BVIs provide a negligible contribution to the overall BVI noise levels.

The mechanics of BVI has been divided into two subsidiary components. The first component which shall be presented is the aerodynamics involved in the problem. The second portion of the mechanics involves the aeroacoustics of the problem, which requires some theory regarding the aerodynamics of the BVI problem to comprehend. As a result, the aerodynamics of the BVI problem shall now be addressed.

\subsection{Aerodynamics of the Blade-Vortex Interaction}

The aerodynamic process of BVI includes several steps which begin as the aforementioned tip vortex is shed. At this point, i.e. the start of BVI, the vortex is upstream of the leading edge (LE) of the next advancing blade and the stagnation point is initially at the LE of the next blade. As the vortex approaches the next advancing blade, a downwash is induced on the blade. This downwash moves the stagnation point along the blades upper surface as a result of the increase in pressure and decrease in velocity over the upper surface. On the lower surface, the flow is accelerated and regions of low pressure may be observed resulting in a decrease of lift. As the vortex passes the LE, an 
upwash is induced on the blade; this causes the stagnation point to move from the upper surface of the blade to its initial location (i.e. the LE) and then towards the lower surface causing a sudden increase in lift. This situation leads to a rapid and continuous change of the airfoils angle of attack (AOA), and highly unsteady airloads. The stagnation point's movement coupled with the effective changing of the AOA influences the time varying lift on the blade. As a result, these are responsible for large unsteady impulsive aerodynamic loads on the rotor blades. Unsteady airloads are one of the mechanisms responsible for blade slap (or discrete frequency noise), a low frequency noise present in $\mathrm{R} / \mathrm{C}$ flight. The rate of change of the lift is therefore associated with the pressure propagated to an observer as the unsteady vortex motion results in pressure fluctuations in the proximity of the blade which degenerates into a series of expansion and compression waves and propagate upstream. Accurate modeling of the BVI problem is thus imperative to achieve accurate predictions related to the rotor airloads and the rotors performance.

BVIs may occur at many different rotor locations and orientations between the blade and vortex axes. If BVI occurs on the advancing side, blade and vortex axes are nearly parallel which promote highly unsteady airloads which in turn results in obtrusive noise with a strongly focused directivity (Schmitz, 1991). BVIs will occur for blade positions

$$
\psi_{b}=\sin ^{-1}\left[\frac{-\cos \Delta \sin \left(\psi_{w}-\Delta\right) \pm \sin \Delta \sqrt{\mu^{2} \psi_{w}^{2}-\sin ^{2}\left(\psi_{w}-\Delta\right)}}{\mu \psi_{w}}\right]
$$


If and only if $\mu^{2} \psi_{w}^{2}-\sin ^{2}\left(\psi_{w}-\Delta\right)>0$ where $\Delta=2 \pi \frac{(i-1)}{N_{b}}$ and $i=1,2, \ldots, N_{b}$. In Equation ( 2.1 ) $N_{b}$ represents the number of rotor blades present, $\psi_{w}$ is the age of vortex element relative to the blade and $\psi_{b}$ represents the position of blade when vortex is formed.

Figure 2.9 illustrates the four types of BVI which occur on the advancing side of the rotor disk (Sim and Schmitz, 2000). Location 1, in Figure 2.4, is the $\delta$ type interaction, in Figure 2.9. The $\delta$ type interaction occurs near the rotors hub for azimuth angles that are less than $30^{\circ}$ and often reach supersonic Mach numbers. The second advancing side interaction, i.e. location 2 in Figure 2.4, is a $\gamma$ type interaction as can be seen in Figure 2.9. According to Lee and Bershader (1994) the $\gamma$ type interaction has the largest trace Mach number, which defines the BVI effect and provides useful information concerning the directionality of the resulting radiated noise (which is defined in Section 2.2 as Equation ( 2.3$)$ ). Also, it generally occurs near an azimuth angle of $45^{\circ}$. In this interaction, 2 BVIs result simultaneously at 2 different blade locations. Each achieve supersonic trace Mach numbers and thence accelerate rapidly together until they meet near the midspan of the blade where the angle between the blade and vortex axes is $0^{\circ}$, i.e. $\gamma=0^{\circ}$. The $\gamma$ type interaction is often referred to as parallel BVI. The net result in the both the near-field and far-field is a relatively intense noise resulting from the phasing of acoustic waves of each rapidly converging BVI event. When $\gamma=0^{\circ}$ the trace Mach number becomes infinite, as is shown by $\gamma$ type in Figure 2.9, this coupled with the phasing effects of the dual BVI results in the greatest noise and vibration. The mechanics 
of the aforementioned BVI acoustic phasing are presented in Section 2.2. Furthermore, $\gamma$ type interactions are the most intense of the interactions illustrated in Figure 2.4, regardless of whether retreating/advancing side BVIs are considered (Yu, 2000) since the blade and vortex axes are aligned. As a result, $\gamma \approx 0^{\circ}$ is the only BVI that provides a significant contribution to the far-field noise produced by a BVI event. The $\beta$ type interaction shown in Figure 2.9 represents location 3 from Figure 2.4. This interaction occurs near an azimuth angle of $55^{\circ}$ and has an effect near the tip which then accumulates to larger values of the trace Mach number near the hub. Finally, location 4 in Figure 2.4 is known as the $\alpha$ type interaction shown in Figure 2.9. The $\alpha$ type interaction reaches its peak effect at an azimuth angle of $70^{\circ}$ and occurs near the blade tip with little effect near the rotor hub. The above classifications of the advancing side BVIs are generalized for all types of R/Cs regardless of the number of rotor blades present in the main rotor as well as the advance ratio of the $\mathrm{R} / \mathrm{C}$.

The positions along the blades which the BVIs given by Equation ( 2.1 ) may be found for $\bar{r} \leq 1$ through

$$
\bar{r}=\frac{r}{R}=\frac{\sin \left(\psi_{b}-\psi_{w}\right)}{\sin \left(\psi_{b}-\Delta\right)}
$$

which gives all BVI intersection points when $\psi_{w}>0$. From Equation ( 2.2 ), the primary sources of BVI noise have been found to occur between $70 \%$ and $85 \%$ of the blade span and from Equation ( 2.1 ) at azimuth angles between $70^{\circ}$ and $90^{\circ}$ azimuth as shown by Martin et al. (1986). On the retreating side, impulsive blade surface pressures are present however measurements have indicated that these BVIs are not a strong source of BVI noise production (Hubbard and Leighton, 1983; Shockey et al., 1976; and Cox, 1977). 


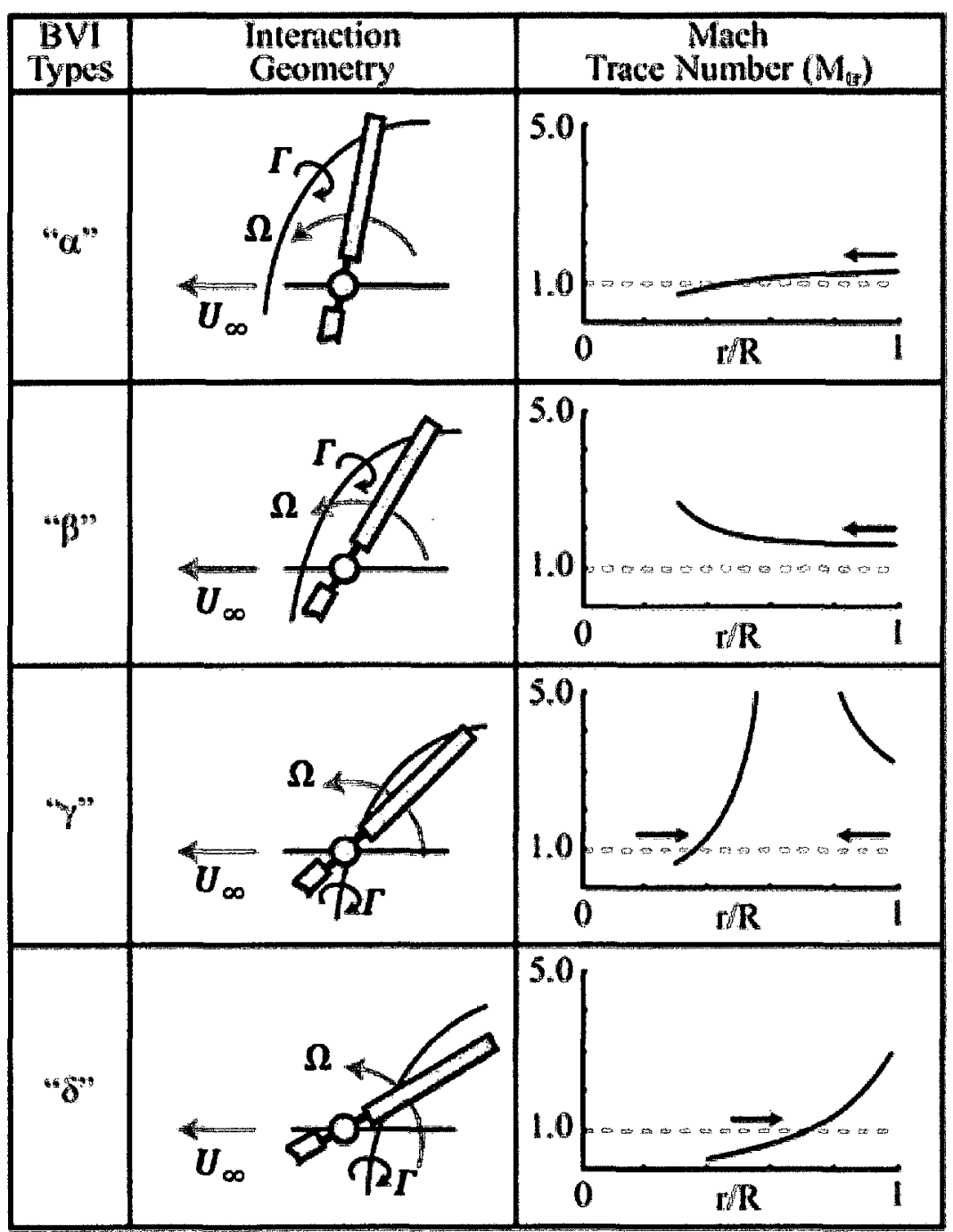

Figure 2.9: Various Types of Advancing Side Blade-Vortex Interactions in Rotorcraft (Sim and Schmitz, 2000)

Most of the aforementioned seven BVIs occur in low speed forward flight. However, as the forward flight speed is increased, the number of BVIs decreases. This happens despite the BVI intensity and the trace Mach number which determines the directivity and noise associated with BVI (Lowson, 1996; and Leishman, 1999). 
The general BVI problem is three-dimensional and unsteady, and the curved line vortex intersects the blade at various angles. The various interactions which are possible in a four-bladed rotor are shown in Figure 2.10.

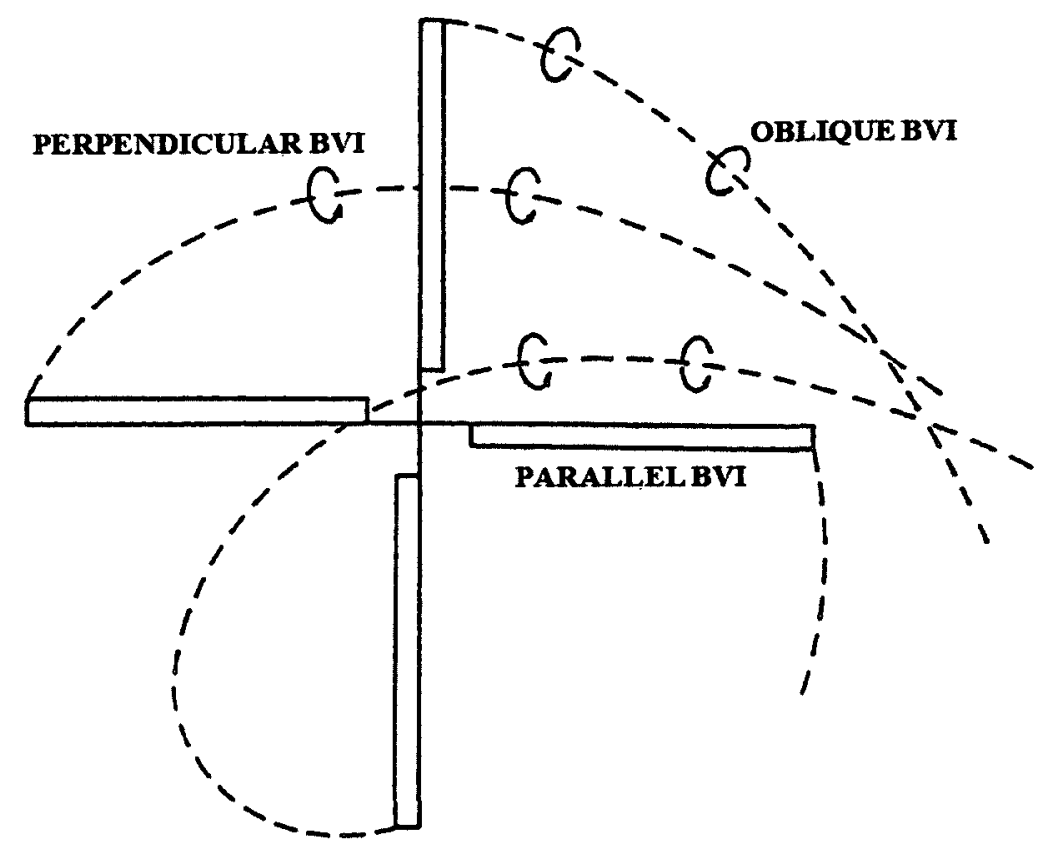

Figure 2.10: BVI for a 4 Bladed Rotor (Renzoni, 1987)

Consideration of the noise propagated to the far-field of a blade-fixed reference frame has shown that blade slap noise is only weakly influenced by the aspect ratio. The parallel interaction produces the greatest total loading on the wing, again with aspect ratio having little effect. Another type which is not indicated in Figure 2.10 is the orthogonal BVI. Orthogonal BVI is the interaction between the main rotors wake and the tail rotor and is responsible for approximately $50 \%$ of the overall noise production of an R/C. It is termed orthogonal BVI as the tip vortices from the main rotor are in a plane which is orthogonal to the plane of the tail rotor. Oblique BVIs are interactions between the blade and vortex for which a head on collision is not imminent, i.e. the vortex has some miss 
distance and is also an event for which the angle between the blade and vortex axes is not $0^{\circ}$ or $90^{\circ}$. Oblique interactions however represent more closely the BVI occurring in an $\mathrm{R} / \mathrm{C}$ during its operation. Two limiting cases of oblique BVI are of fundamental interest. One such case occurs when the axis of the vortex is along the freestream and the vortex is normal to the blades leading edge (LE), and is referred to as perpendicular BVI. The other limiting case occurs when the vortex is parallel to the blades LE. It is known as parallel BVI and can be analyzed from a two-dimensional perspective of the full three dimensional BVI problem. Thus, parallel BVI is synonymous with the term airfoil-vortex interaction (AVI). An AVI investigation provides useful information pertaining to the mechanics involved in the transfer of energy to the blade including the resulting vibration and noise. Although AVI does not completely describe its BVI counterpart, it remains a valuable investigative tool which is used to gather otherwise complex and difficult to obtain data. AVI accurately describes the aerodynamics (Srinivasan, 1986) within the plane perpendicular to the blade and vortex axes during the event. The orientation of the blade and vortex axes in AVI is the most intense type of BVI, and is therefore of interest in considering noise and blade fatigue. As a result, $\gamma=0^{\circ}$ (i.e. AVI) is the focus of the current study. Figure 2.11 (Srinivasan, 1986) presents the plane and side view of the typical path of a vortex during parallel BVI (i.e. AVI) where $y_{v}$ represents the vertical miss distance of between the blade and vortex axes, and $\Gamma$ represents the circulation of the vortex. Radiated noise is dependent on $y_{v}$ and the characteristics of the tip vortex shed off the previous blade. 
The aeroacoustics of the BVI problem shall now be addressed, and is based on the aerodynamics of the problem which has been presented in the current section.

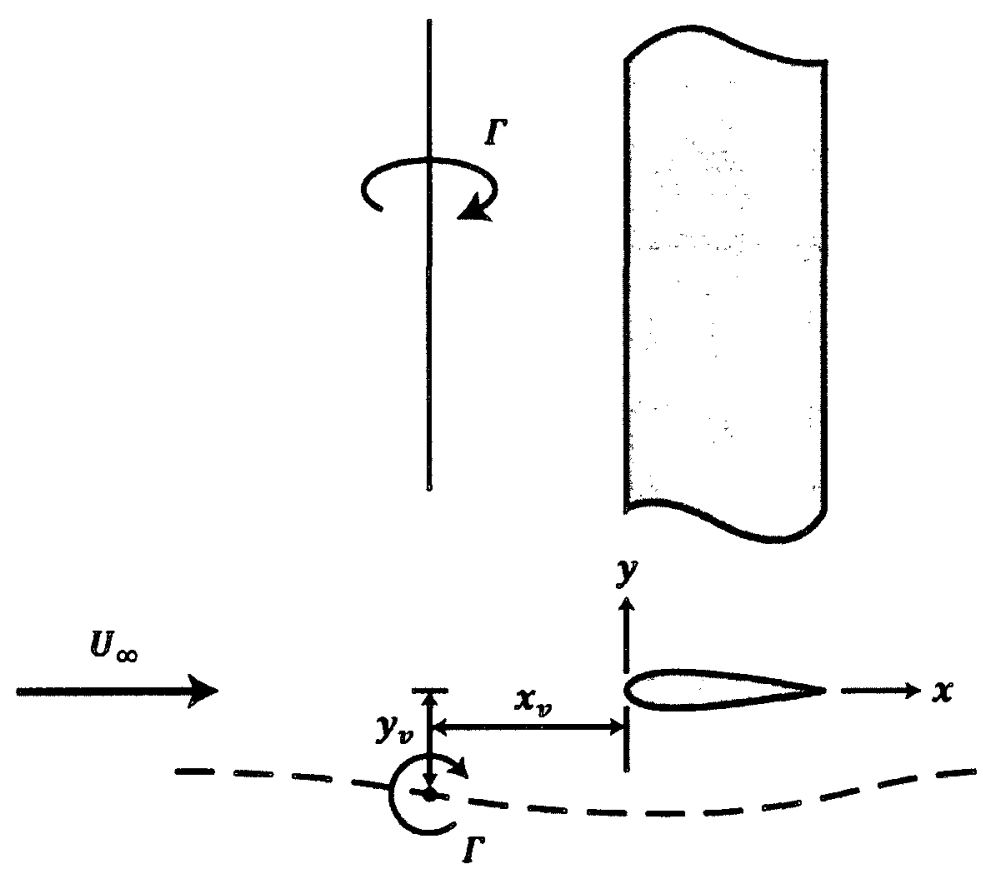

Figure 2.11: Path of a Vortex with Respect to the Airfoil in the Parallel Blade-Vortex Interaction Case $\left(\gamma=0^{0}\right)$ (Srinivasan, 1986)

\subsection{Aeroacoustics of the Blade-Vortex Interaction}

$\mathrm{R} / \mathrm{C}$ rotor noise levels tend to be concentrated at the harmonics of the blade passage frequency, $N_{b} \Omega$ due to the periodic nature of the rotor with respect to a nonrotating frame of reference, as can be seen in Figure 2.12. 


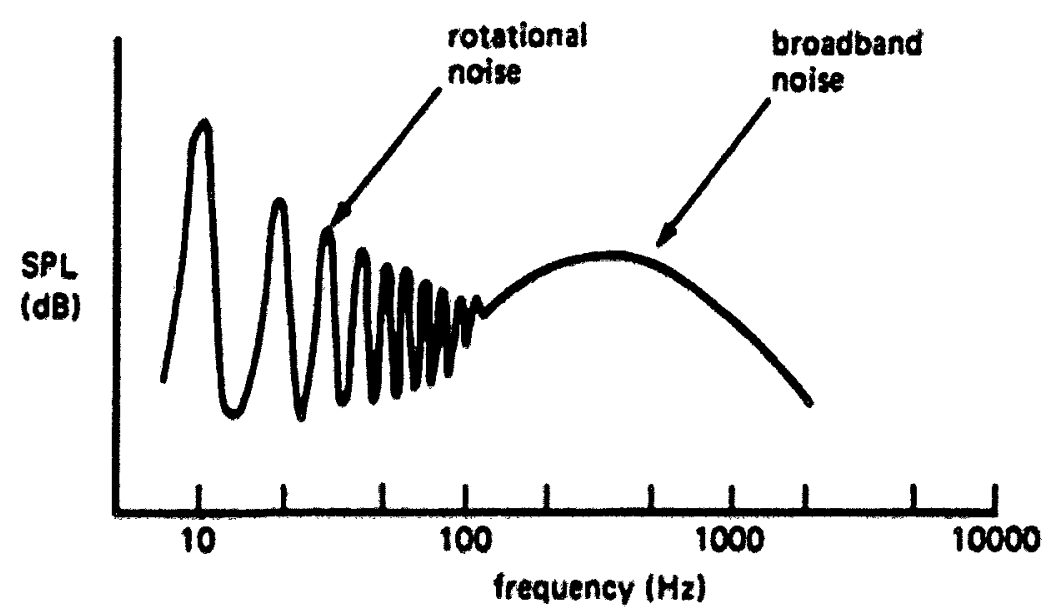

Figure 2.12: Helicopter Rotor Sound Spectrum (Johnson, 1980)

The sound which is radiated is a result of the mean thrust and drag forces that rotate with the blades as well as of the higher harmonic loading. The spectral lines are broadened at higher harmonics due to the random character of the rotor flow, especially with respect to the variations introduced by the wake-induced loads. The acoustic pressure signal is nearly periodic, with a period of $2 \pi / N_{b} \Omega$, and has sharp impulses from various localized aerodynamic phenomena, such as compressibility effects and vortex-induced loads. Therefore, the contributions to the $\mathrm{R} / \mathrm{C}$ rotor noise can be classified as vortex (or broadband) noise, rotational noise and blade slap (i.e. extreme of vortex noise). Listed in terms of the level of intensity of the noise sources, these are blade slap, vortex noise and then rotational noise.

Vortex (or broadband) noise is characterized by a high frequency swishing sound produced by the rotor. It is modulated in both frequency and amplitude at the blade passage frequency. In broadband noise, a random sound is radiated from random fluctuations of the forces on the blades. The sound energy is distributed over a large 
portion of the audible noise spectrum. For main rotors it is typically between 150 and $1000 \mathrm{~Hz}$, with peaks near 300 to $400 \mathrm{~Hz}$. Vortex noise is principally produced by random fluctuations in lift with the blade operating in the rotors turbulent wake, specifically due to random blade loads induced by tip vortices. Other sources of vortex noise include forces on blade due to vorticity shedding from the trailing edge of the blade, turbulence in the freestream, and boundary layer turbulence and transition. When the sound level is corrected for frequency content, often vortex noise dominates all the sources.

Rotational noise is the primary determinant of the overall sound pressure level. However, it is not the most important source of noise in terms of annoyance. It can be important when its sound pressure level increases at high frequency, i.e. blade slap is approached. In particular at high tip speed with a low number of rotor blades, $N_{b}$. Rotational noise may produce acoustic fatigue and vibration of the $\mathrm{R} / \mathrm{C}$ structure. Rotational noise is characterized by a thumping sound either at the blade passage frequency or at $N_{b} \Omega$ if the fundamental frequency is inaudible. As the higher harmonic content increases, the thumps sharpen into bands and eventually may become blade slap. The spectrum in which rotational noise occurs varies greatly with both the rotors geometry and the operating condition of the R/C. The sound pressure is purely periodic and is caused by the periodic forces exerted by the blade on the air through a rotor revolution. The spectrum of noise from rotational noise consists of discrete lines at the harmonics of the blade passage frequency, i.e. $N_{b} \Omega$. Rotational noise dominates the low frequency end of the spectrum. For main rotors, it ranges from below audible frequencies to about $150 \mathrm{~Hz}$. The fundamental frequency, $N_{b} \Omega$, for a main rotor is typically between 
10 and $20 \mathrm{~Hz}$. In this range, the fundamental frequency and perhaps its first and second harmonics are below the hearing threshold. Since the lift and drag forces rotate with the blade, rotational noise is produced by forces being periodically exerted on the air at any fixed point of the rotor disk. The higher harmonic blade loading is responsible for the large high frequency content of $\mathrm{R} / \mathrm{C}$ rotational noise.

Blade slap is characterized by a sharp cracking, popping or slapping sound at $N_{b} \Omega$ and is produced by the main rotor in certain flight conditions. Blade slap occurs most often in maneuvers (from flare to landing, shallow descents, and decelerating steep turns). It may also occur at high forward flight speeds and level flight at moderate speeds blade slap. Blade slap is caused by a periodic and impulsive sound pressure disturbance. In other words, it is an extreme case of rotational noise. The impulsive character of blade slap leads to an increase of the sound level over the entire spectrum, i.e. 20 to $1000 \mathrm{~Hz}$ for the main rotor. Blade slap is the dominant noise source with a high overall sound pressure level and its impulsiveness makes it the most objectionable of the noise sources as well. As such aerodynamic phenomena produce large, localized transient forces on the blades which in turn results in impulsive sound radiation, the most likely source of blade slap is BVI. Low frequencies propagate best in air and high frequencies are best attenuated as the distance from the source is increased. Therefore, at very large distances from the R/C, blade slap and rotational noise of the main rotor are the most important sources of noise to consider. As a result, the acoustic detection of a helicopter is often determined by rotational noise and its special case, blade slap. It is an impulsive type of sound pressure disturbance which occurs at $N_{b} \Omega$. Blade slap is best investigated in the 
time domain due to the impulsive nature of the sound pressure. The cause of blade slap is any aerodynamic phenomena which produces rapidly changing loads on the blades. Its occurrence is sensitive to rotor design parameters and the operating condition of the R/C. Increasing the overall sound pressure level, results in an increase of the noise spectrum level over a wide range of frequencies this results in the impulsive nature of blade slap as well as the associated annoying sound.

In flight conditions which involve close encounters of the blades with their own wake or the wake of another rotor, BVI is an important source of blade slap. A large portion of noise produced by an $\mathrm{R} / \mathrm{C}$ is generated by unsteady aerodynamic forces, including the interactions of a blade with the wake and/or the discrete tip vortices (i.e. the BVI problem) (Widnall, 1971; George, 1978; Schmitz and Yu, 1986; and Schmitz, 1991). The aerodynamic intensity of a BVI event depends on the strength of the tip vortex, the distance from the blade to the tip vortex and the orientation of the vortex with respect to the blade. Depending on the orientation of the vortex and blade axes during the interaction, the magnitudes of noise and vibration from the resulting BVI vary. The highly three-dimensional unsteady aerodynamics produced by the various BVI events on the blades give rise to multiple noise sources with differing directivity and phase relationships. The net sound field therefore comprises of many complicated interfering omni-directional traveling sound waves and often highly focused acoustic wave paths are also produced. BVI noise has a pronounced directivity. As a result, there is a possibility of localized regions that experience sound focusing effects. Particularly, BVI noise is more pronounced when the LE of the blade is parallel to the axis of the vortex on both the 
advancing and retreating sides of the rotor disk in forward flight. Also, the BVI noise problem is especially pronounced during descending low speed forward flight or maneuvering flight, i.e. flight conditions where the tip vortices lie closer to the rotor.

As a series of discrete directions since the resulting acoustic waves may accumulate along a front, an acoustic field generated by an $\mathrm{R} / \mathrm{C}$ often tends to be highly focused in one specific direction. The amount of focus depends on the trace Mach number of the acoustic source point. The trace Mach number defines the BVI effect and is useful information on the directionality of the resulting radiated noise (Srinivasan, 1986) and is defined as

$$
M_{t r}=\frac{\Omega r_{b}+U_{\infty} \sin \left(\psi_{\mathrm{b}}\right)}{a \tan (\gamma)}
$$

The trace Mach number, $M_{t r}$ can range from supersonic to subsonic and the trace velocity vector, $\Omega r_{b}$, can be directed inward or outward along the blade axis. For instance, if the intersection angle is small (i.e. the blade and vortex axes are near parallel), the trace Mach number can be substantially larger than one (i.e. supersonic) as illustrated by the $\gamma$ type interaction in Figure 2.9. A diagram explaining the basic concept of the trace Mach number is given in Figure 2.13 for an axis system which moves with the rotor. 


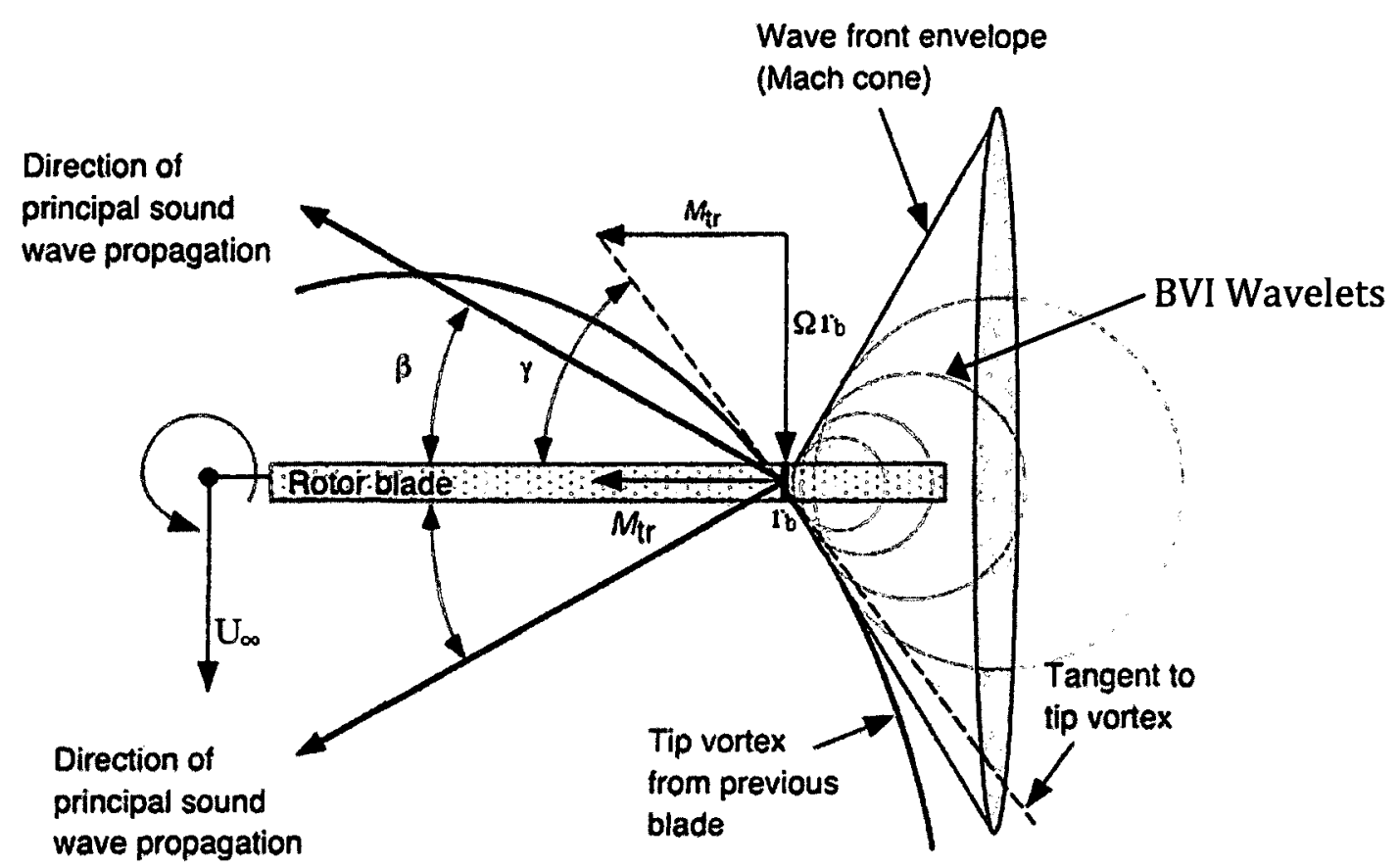

Figure 2.13: Ideas of the Trace Mach Number of an Acoustic Source Point on the Rotor (Leishman, 2006)

Under many conditions, the trace Mach numbers of the BVI intersection points (i.e. the source points) between the blade and vortex filament axes inside the rotor disk are supersonic. Consequently, the fronts of spherical sound wavelets generated at BVI source points on the blades will accumulate along an envelope, which is similar to a Mach cone as is shown in Figure 2.13. Furthermore, the principal direction of sound propagation will be normal to this Mach cone. Sound waves that have originated from a cluster of source points with supersonic trace Mach numbers on the rotor will arrive simultaneously at the same observer location, thus providing acoustic convergence. This effect is similar to the wave on a ground plane from supersonic fixed-wing aircraft which generates a sonic boom. Figure 2.13 also demonstrates that the accumulated wavelet 
fronts will propagate into the acoustic field along a ray cone with semi-vertical angle, $\beta$, to instantaneous trace velocity vector, $\Omega r_{b}$, where

$$
\beta=\cos ^{-1}\left(M_{t r}^{-1}\right)
$$

The ray cone semi-vertical angle, $\beta$, varies from point to point along the blade and forms a series of intersecting ray cones with different vertices and spreading angles. When the ray cones are generated by each supersonic BVI source point in the rotor plane intersect the horizontal ground plane they become conics. Since this intersection forms the locus of any acoustic disturbance from wave fronts reaching the observer plane from the BVI emission points, this planar intersection is termed as acoustic lines. After the wavelets intersect the observer plane, the principle direction of the front is along the acoustic lines. If the wave fronts intersect approximately in regions of high acoustic line density, sound may focus. Depending on the trace Mach number and the direction of the trace velocity vector along blade, the resulting acoustic lines may crowd together or overlap. A possible result is that sound may focusing in regions of high acoustic line density (in some cases they may converge to form caustics (i.e. the intersection of ray cones) which causes wave focusing and the formation of acoustic hot spots. When an $\mathrm{R} / \mathrm{C}$ is in forward flight, the radiation cones become distorted by the low local flow velocities.

Wave fronts of focused acoustic energy found at the intersection of close spherical wavelets are generated at each BVI source point with supersonic trace Mach numbers. On the observer plane, two sets of circular wavelets generated: one from the BVIs at the rear of the disk and one from the BVIs at the front (these occur $1 / 2$ a revolution apart). The primary wave fronts formed by the intersection of the individual 
groups of wavelets from each blade traveling along paths are defined by two sets of acoustic lines. These lines result in the sound wavelets generated by a parallel BVI event becoming highly focused in direction perpendicular to blades at azimuth angles of $0^{\circ}$ and $180^{\circ}$ (i.e. the rear and front of the disk respectively). When the blade and vortex axes are parallel, there is one BVI event and all points on blade produce in-phase sound waves. Moreover, the duration and phasing of BVI along blade, Doppler magnification, and distance from an event to the microphone location combine to produce net sound pressure signature at a given time. The thickness of the sound pressure combines with the loading term resulting in small variations in the phasing which may affect the rotors net noise signature. Both the near and far field acoustics are sensitive to the phasing of the aforementioned unsteady airloads during a BVI event.

Large majorities of the BVIs on the rotor involve oblique interactions the acoustic effects must be addressed. Aerodynamically, oblique BVI events produce significant three-dimensional unsteady airloads. Acoustically, the obliqueness of BVIs results in complicated acoustic directivity. Since the BVIs are no longer parallel events, there are fewer points along the blade with supersonic trace Mach numbers. This results in the existence of varying supersonic trace Mach numbers which results in the focused sound waves radiating over a larger spread angle for oblique BVIs.

BVI is assumed to be chordwise compact. Since the acoustic signature radiates outward spherically at each intersecting point of the blade and vortex through time, the integrated effect is presumed to act at the $1 / 4$ chord location of the airfoil. Thus, certain areas in the near and far field exist which experience constructive interference of the 
temporally and spatially separated wavelets emanating from the intersection of the $1 / 4$ chord line of the blade and vortex axes. Amplification of these sound waves results in the characteristic chopping sound of BVI noise which is also potentially related to the highly impulsive changes in pressure.

The investigations, both numerical and experimental, concerning BVI are numerous and shall now be addressed.

There are currently numerous methods which are used to predict the rotor acoustics of a R/C. The first is a purely empirical approach, followed by wave tracing methods. A blade element type unsteady aerodynamic model coupled with the FfowcsWilliams-Hawkins (F-WH) methods is another alternative. Recently computational fluid dynamics simulations regarding acoustic predictions have also been performed.

The investigations, both numerical and experimental, concerning BVI are numerous and shall now be addressed.

\subsection{Investigations Concerning the Blade-Vortex Interaction}

The current state of BVI research from a numerical and experimental stand point shall now be addressed. The vast majority of numerical and experimental investigations for a blade or an airfoil have utilized a NACA 0012 airfoil at zero AOA as a test rotor, with some cases exploring situations where the AOA is larger than zero. Many vortex generation methods, both for numerical and experimental investigations, employed a NACA 0012 airfoil or a NACA 0018 airfoil with a pitching or oscillating motion or a shock tube technique. All of these methods relied on the concept of a startup vortex as the 
means of vortex creation. The experimental investigations concerning BVI shall now be addressed.

\subsubsection{Experimental Investigations in Blade (Airfoil)-Vortex Interaction}

The methods invoked to recreate the vortex in wind tunnels vary from moving airfoils (pitching (Straus et al., 1990; Kalkhoran and Wilson. 1992; and Seath et al., 1989) or oscillating (Booth and $\mathrm{Yu}, 1986$; and Booth and $\mathrm{Yu}, 1990)$ ) to stationary airfoils (shock tube (Lee and Bershader, 1994) or blade tip (Caradonna and Strawn, 1988)) at some distance upstream of the test airfoil. Vortex generation occurs at a forward airfoil, which is usually a NACA 0012 or NACA 0018 profile. The vortex is then allowed to convect downstream as occurs in numerical simulations. Experimental investigations in both the subsonic (Seath et al., 1989; Lee and Bershader, 1994; Straus et al., 1990; Kalkhoran and Wilson, 1992; Booth and Yu, 1986; Caradonna and Strawn, 1988; and Booth and Yu, 1990) and transonic (Kalkhoran and Wilson, 1992; and Caradonna and Strawn, 1988) flow regimes have been performed. Experimental work mainly exploits the 2D nature of the parallel BVI measure since it is a simpler event to measure. Lee and Bershader (1994) demonstrated that viscous effects play a significant role in parallel BVI by using a dual pulsed holographic interferometer with blade pressure measurements. $\mathrm{A}$ vortex was created with a fixed highly pitched stationary NACA 0018 airfoil interacting with a shockwave to exploit the starting vortex caused by the step change in lift. Vortices created using the bound vorticity shed as a starting vortex from a rapidly pitching airfoil are commonly employed. Seath et al. (1989) used an impulsively pitching airfoil upstream of the test airfoil with numerous pressure taps on both the upper and lower 
surfaces for relevant measurements, in a subsonic wind tunnel. The effects of the maximum pitch angle on the resulting vortex as well as that of the vortex position and freestream velocity were also included in this investigation. Straus et al. (1990) pitched a NACA 0018 airfoil to create a vortex which was then measured with hot-wire anemometry. It was determined that inviscid theory accurately only predicts the pressure distribution on the test airfoil for specific situations. The validity of ignoring viscous effects in numerical BVI studies was therefore questionable. The inviscid theory that was used to compare the experimental results obtained by Straus et al. (1990) was presented by Renzoni (1987). Renzonis theory investigated a parallel interaction using a discrete free-vortex method which was developed. It was found that the changes in lift and moment coefficients during the interaction may expressed as incremental coefficients. The incremental coefficient formulation was further expanded upon by Renzoni and Mayle (1989). This investigation correlated the incremental coefficients based on different miss-distances and strengths of the vortex as well as airfoil angles of attacks. It was also found that the incremental lift coefficient for a close encounter was approximately four times the non-dimensional circulation. Kalkhoran and Wilson (1992) performed 2D transonic wind tunnel tests in order to collect target rotor pressure measurements for various $y_{v}{ }^{\prime}$ s using a pitching NACA 0012 for vortex generation. An oscillating NACA 0012 airfoil has been used to create a street of counter-rotating vertical structures in a subsonic wind tunnel by Booth et al. $(1990,1986)$ to perform BVI measurements at different $y_{v}$ 's. Flow visualization using smoke, a strobe light and still photography was used. It was found that the vortex trajectory was greatly influenced at 
small $y_{v}{ }^{\prime} \mathrm{s}$ and that the vortex core experiences massive distortion at small $y_{v}{ }^{\prime} \mathrm{s}$. Caradonna and Strawn (1988) performed a fully 3D BVI investigation using a true tip vortex method to introduce the vortex into the flow. To produce the tip vortex a NACA 0015 airfoil was pitched perpendicularly upstream of a spinning 2 bladed test rotor, as is illustrated in Figure 2.14. The tip vortex was formed at the end of the vortex generating NACA 0015 airfoil and convects downstream to the test rotor where a number of BVI measurements, using pressure transducers on the rotor blades, may be performed depending on the location of the test rotor setup. The steady vortex sheet had a nondimensional circulation of $\bar{\Gamma}=0.406$ and a non-dimensional core radius of $\bar{r}_{c}=0.379$.

Although clear experimental studies of parallel BVI are rare, good but idealized quantitative measures of BVI collisions have been described by Horner et al. (1994) and Kitaplioglu and Caradonna (1994).

Several visualization methods have been employed by numerous researchers. For example, visualization of the problem has been performed with smoke by Mercker and Pengel (1992) and Lorber et al. (1994) and with strobed shadowgraphy by Swanson (1993). 


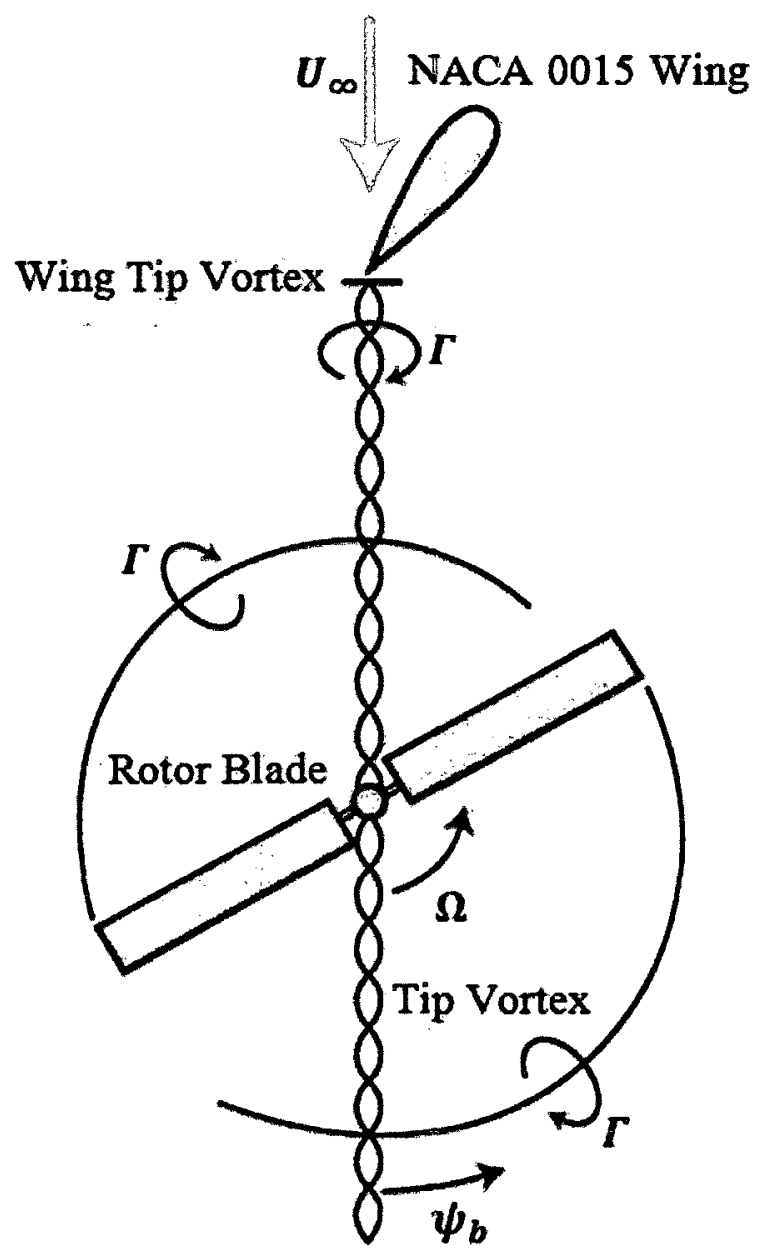

Figure 2.14: Schematic for wind tunnel rotor BVI test by Caradonna and Strawn (1988)

Another visualization technique which also provides quantitative data employed in BVI research is particle image velocimetry (PIV). PIV is a non-intrusive technique in which a relatively high frequency of repeated measurements can be made, and lends itself to the application of BVI measurements. A three-dimensional BVI study, was performed by Raffel et al. (2004) using three-dimensional PIV. Furthermore, a proof-of-concept investigation for three-dimensional PIV BVI measurements using seed particles was conducted. The particles were composed of Di-ethyl-hexyl-sebacat (DEHS) with diameters of less than 1 micrometer and were created with a Laskin nozzle generator 
which released the seeding particles into the flow upstream of the target test area. During the measurements, a decrease within seeding density in the vortex core was a result in the decrease of air density in the core and the inertial forces on the seeding particles in the swirling vertical flow. To perform measurements of the event, a head on parallel BVI PIV investigation by Horner et al. (1996) used seed particles composed of expancelDE20 micro balloons with a mean diameter of 20 microns and a specific gravity of 0.06 . The measurements indicated a strong distortion of the vortex which had good agreement compared to surface pressure measurements. Green et al. (2000) aimed at increasing the PIV measurement accuracy of BVI. This was done by employing two separate PIV systems which acquired measurements in very close temporal proximity to each other. The available number of measurements doubled; and as a result the chances that each time a measurement was made with the vortex core at a desired location due to timing increased. Measurements were conducted in a low speed wind tunnel using a vortex generator defined by Doolan et al. (1999). Capturing an image pair of the vortex at exact (or near) locations for hundreds of measurements required for averaging because a problem with PIV measurements is this experiment was performed. Grant et al. (1994) devised a geometrical method to alleviate the challenge for PIV measurements and improve their accuracy when a strong out-of-plane fluid motion is present. This problem was addressed since many BVI types' flows are not constrained to two-dimensions as is the case for parallel BVI. Green et al. (2005) studied the flow around a sinusoidally pitching symmetric airfoil with PIV capturing the vertical flows brought on by oscillatory pitching motion. The seeding was performed with 11 micron hollow glass spheres in a 
water tunnel. The final vector field was comprised of 100 averaged instantaneous flow measurements. Burwash (2007) used PIV to obtain the measurements of an oblique BVI, a wind tunnel was created specifically to capture the event created using a pitching airfoil upstream of the test airfoil.

Several idealized BVI experiments have also been conducted concerning R/C aeroacoustics (Surendraiah, 1970; Seath et al., 1987; Kokkalis and Galbraith, 1986; Kitaplioglu and Caradonna, 1994; and Kitaplioglu et al., 1997) that provide valuable measurements for validation of aeroacoustic models. A model for an idealized BVI aeroacoustic situation using an elastically stiff two bladed rotor encountering an idealized streamwise vortex parallel to $\mathrm{x}$-axis with results for both parallel and oblique (or offset) cases was examined experimentally by Kitaplioglu and Caradonna (1994). In this study, both the unsteady blade loads and acoustics data was collected where the hover tip Mach number was 0.7 and the advance ratio of the model was 0.2 . The primary BVI event occurs over the front of the rotor disk where the blade axis is parallel to the axis of the vortex generator. While a BVI event may be expected downstream as well, the hub rapidly diffuses the vortex and eliminates the potential BVI at an azimuth of $0^{\circ}$. The test section employed is depicted in Figure 2.15. 


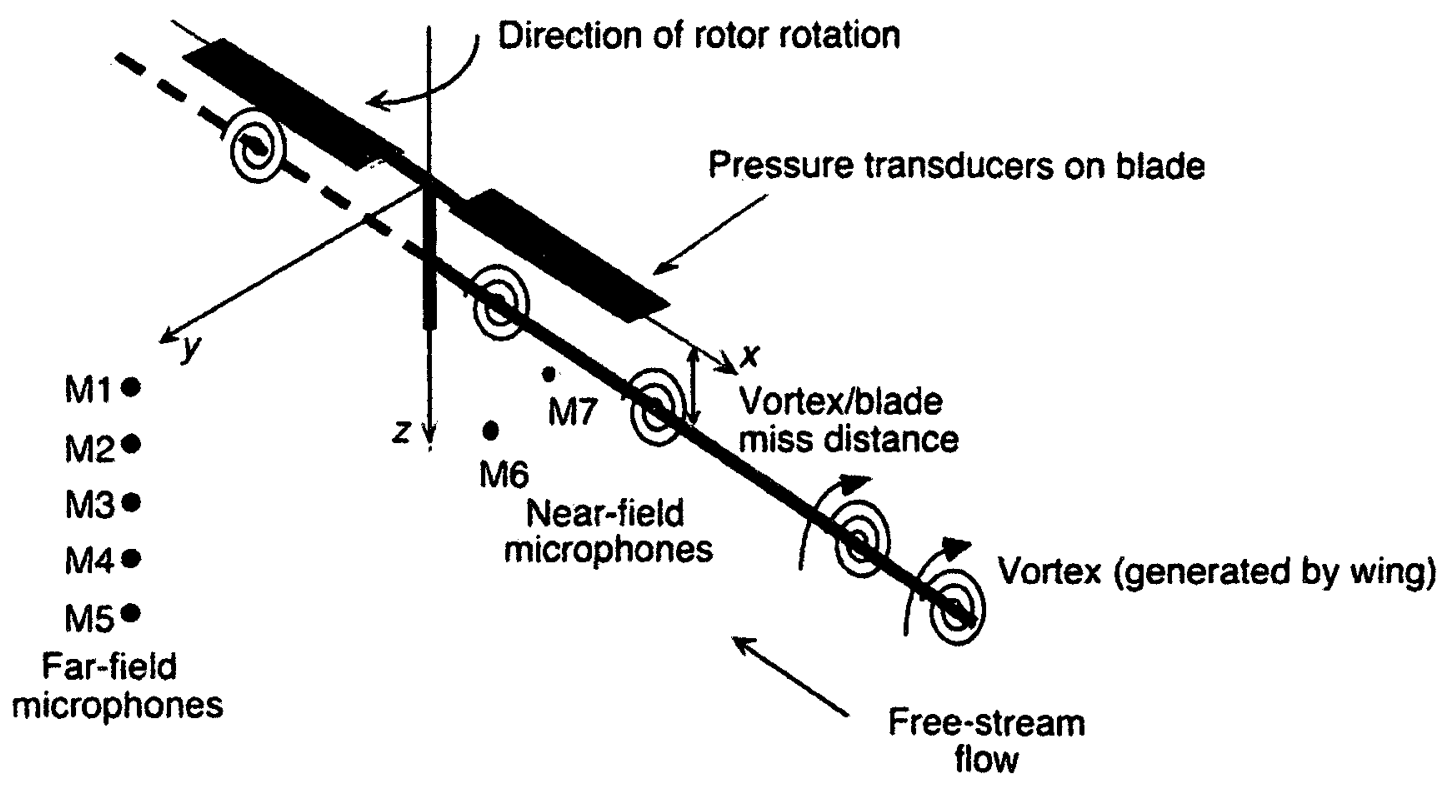

Figure 2.15: Schematic of the Configuration Employed in the Experiment Conducted by Kitaplioglu and Caradonna (1994)

Caradonna et al. (1997) compared experimental data for parallel interactions $\left(y_{v}=0\right)$ as well as oblique interactions $\left(y_{v}=0.3\right)$ with predictions from various types of acoustic models. As illustrated in Figure 2.16a, the first case involved acoustic lines from BVI sources on the rotor with supersonic trace Mach numbers compared with the F-WH solution for an observer plane 3 rotor radii below the rotor disk for the parallel interaction. The second case, depicted in Figure 2.16b, involved the same comparison for the oblique interaction. 
(a) Parallel blade vortex interaction $y=0.0$
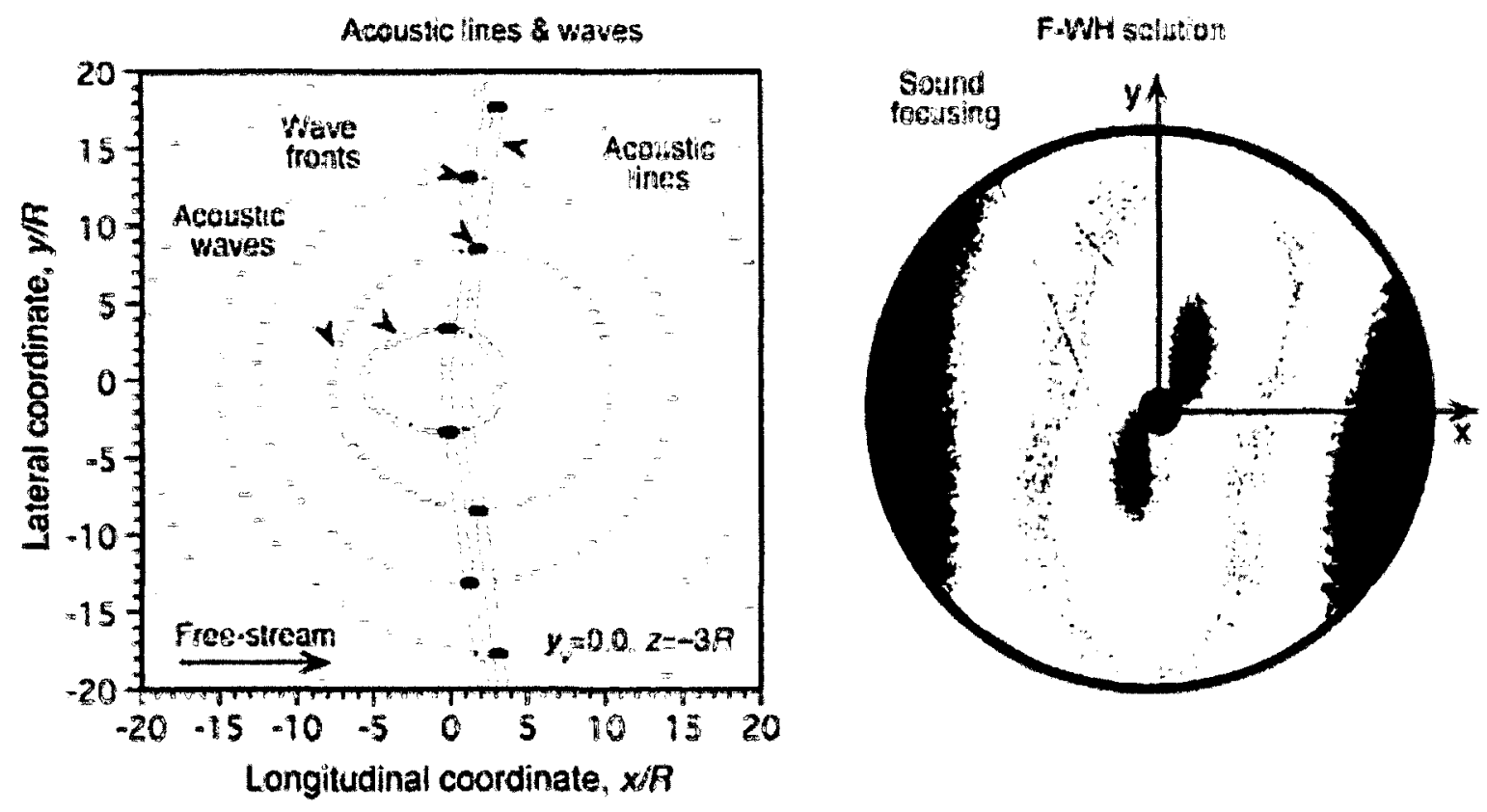

(b) Oblique blade vortex interaction, $y=0.3$
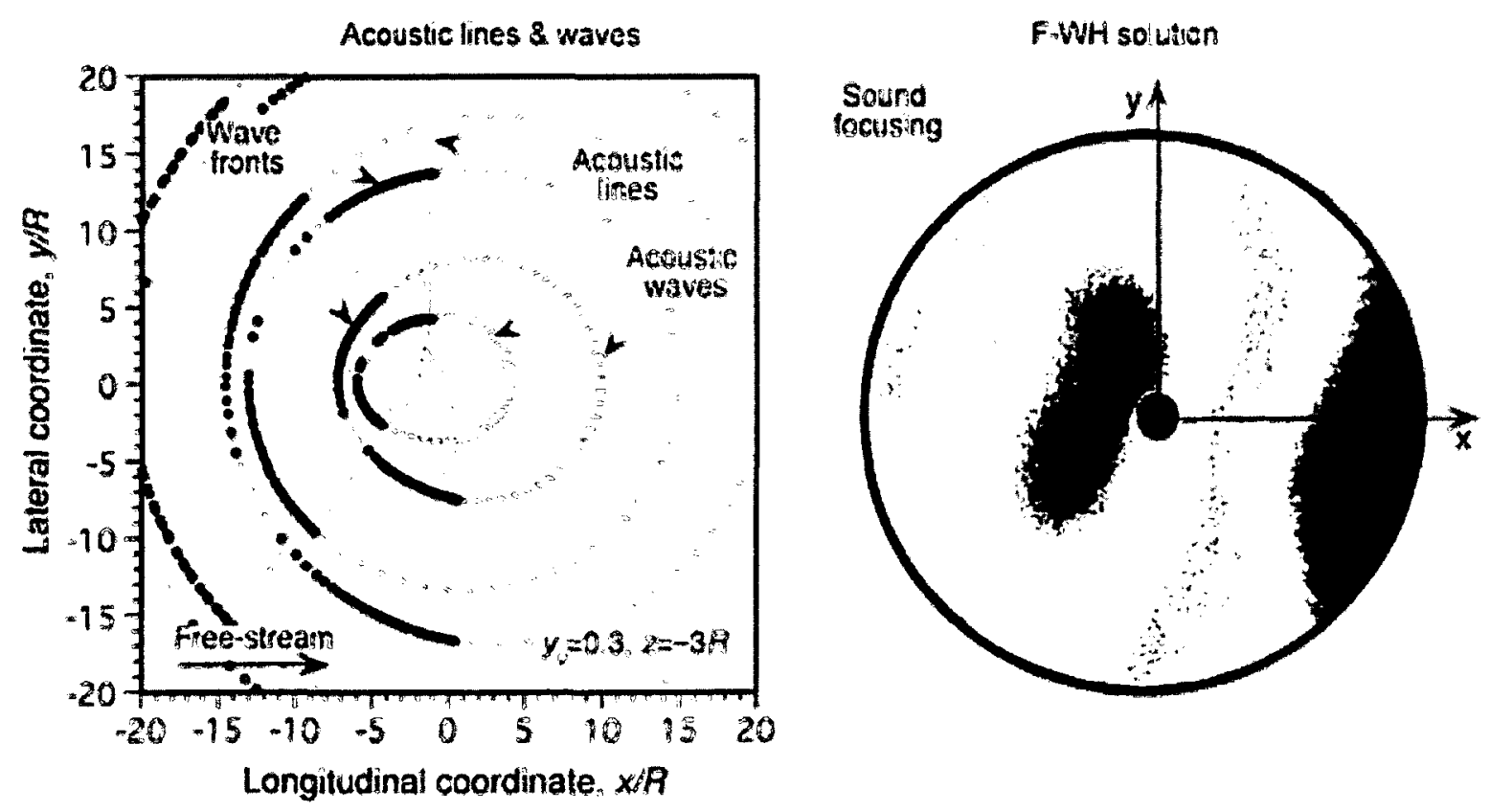

Figure 2.16: Acoustic Results for a Two-Bladed Rotor (Experimental and Numerical) for a) a Parallel Interaction, and b) an Oblique Interaction (Kitaplioglu and Caradonna, 1994) 
In the study performed by Caradonna et al. (1997), wave tracing techniques were used in order to determine the principal sound direction and arrival time from all BVIs with supersonic source points. In the parallel case, shown in Figure 2.16a, the trace Mach number was found to become infinite all along the blade for azimuth angles of $0^{\circ}$ as well as $180^{\circ}$. Therefore, the vertex of each ray cone was located on the horizontal axis upstream and downstream of the rotational axis. For the oblique case, however, as illustrated in Figure $2.16 \mathrm{~b}$, the acoustic lines were all directed towards the front of the rotor disk.

Cox and Lynn (1962) found that the occurrence of blade slap is associated with significant vortex induced loads through rotor airload measurements. From these measurements, it was concluded that slap at low flight speeds was due to BVI on the advancing side, and also that there exists a dependence of slap on flight conditions, therefore resulting from variations of the wake geometry. Also, blade slap at high speeds was found to be caused by the formation of local shockwaves on the advancing tips (Cox, 1963).

Schlegel et al. (1966) determined that blade slap was a high amplitude rotational noise with a highly modulated broadband noise component. Blade slap is also associated with blades retreating as a result of stall. Local shockwaves were produced at drag divergence locations which in turn, causes a portion of the blade slap noise.

A BVI encounter, simulated by directed jets at the disk plane of a hovering rotor in order to approximate the downwash profile of a vortex, was performed by Leverton and Taylor (1966). Both parallel and perpendicular BVI were simulated through this 
experiment. It was found that both the spectrum and time history of noise produced correlated well with actual $\mathrm{R} / \mathrm{C}$ blade slap. The interaction of the blades with the tip vortices from the preceding blade was found to be the likely source of blade slap. It was also determined that the SPL scales with the $4^{\text {th }}$ power of the tip speed and the square of the vortex strength, i.e. $p^{2} \propto(\Omega R)^{4} \Gamma^{2}$

Leverton (1968) concluded that the sound power, $W_{b}$, scales as $W_{b} \propto(\Omega R)^{4} \Gamma^{2} \propto$ $\frac{(\Omega R)^{2} T^{2}}{N_{b}^{2} A}$ since the tip vortex strength, $\Gamma$, is proportional to $T / \rho N_{b} \Omega R^{2}$ (where $T$ represents the rotor thrust).

Bausch et al. (1971) performed an experimental investigation of the impulsive noise of a rotor in both hover and forward flight. The impulsive noise in hover was found to be due to high frequency airloads produced by BVIs. Also, the investigation determined that blade slap in forward flight is caused by the combination of high advancing tip Mach number on the propagation of acoustic waves which in turn effects the drag.

Sternfeld et al. (1972) investigated the rotor impulsive noise generated during hover. They determined that the tip vortex from the blade produces transient flow changes at the following blades, this change produces a shock wave or moves existing shocks. The pressure disturbance due to this localized phenomena results in blade slap. It was also determined that when an airfoil with a high divergence Mach number was selected, the effect of blade slap was decreased. 
Isom (1975) examined the scaling of noise from a hovering rotor with a high tip speed (i.e. high $M_{t i p}$ ). In this study, the sound contributions from the thickness, lift, drag, and shockwaves were considered. A narrow zone of intense noise carried by each blade tip was identified, which would produce an $N_{b} / \mathrm{rev}$ impulsive noise at a fixed observation point. It was noted that the lift dominates the rotational noise and the thickness is an important parameter for the impulsive noise generated.

Farrasat (1975) demonstrated that the thickness noise (a type of rotational noise) of the rotor exhibits characteristics of blade slap. The thickness noise generally consists of impulsive acoustic pressure with a sharp negative peak at high Mach numbers. As a result, it was concluded that the thickness noise strongly depends on the Mach number and has a maximum directivity in the plane of the rotor disk.

Boxwell et al. (1975) gathered in-flight measurements of R/C impulsive noise. From these measurements three distinct types of impulsive noise which occur sequentially in the pressures time history were identified. The first type of impulsive noise is represented by a series of positive pressure pulses at all speeds, but is sensitive to the R/Cs descent rate and was found to be associated with BVI. The second type of impulsive noise has a negative pressure distribution with directivity that is concentrated on the disk plane. The amplitude of the pulse was found to increase with forward flight speed until at the maximum speed, when it dominated the pressure signal. It is also determined to be associated with compressibility effects. As a result, pulse amplitude is dependent on the Mach number. The final type of impulsive noise occurs only at high 
flight speeds and is represented by a narrow positive pressure spike which is followed closely by a negative pulse.

Based on both experimental and numerical investigative techniques, rotor noise reduction or defocusing has also been a current research topic within the $\mathrm{R} / \mathrm{C}$ community. Several techniques which have been investigated shall now be presented.

\subsubsection{Investigations Concerning the Reduction of the Noise Produced by a Blade- Vortex Interaction Event}

Other than the experimental investigations related to BVI aerodynamics and aeroacoustics, the reduction of the BVI effect has also been a current focus of helicopter research. The quest to decrease $\mathrm{R} / \mathrm{C}$ noise requires an accurate prediction of the rotor noise intensity and directivity as well as strategies which reduce or defocus the rotor noise. Consequently, rotor noise reduction is the current focus of research being performed in the R/C aeroacoustics field (Brentner, 1997(a,b); and Edwards and Cox, 2002).

Through experimental and numerical studies, the most significant factors of BVI noise include, the miss distance, $y_{v}$, along with the characteristics of the vortex (Hardin and Lamkin, 1997; McAlister, 1996; and Yu, 2002). The airfoil AOA influences both the aerodynamic coefficients and the aeroacoustic field (Ilie et al., 2008). However, the tip speed has been determined to be the most important factor for the determination of the rotor noise level.

All attempts to reduce the rotor noise have either proposed modification of the core structure of the tip vortex, alteration of the tip vortex trajectories, or various 
mechanical changes to the rotor. All three types of noise produced during the operation of an $\mathrm{R} / \mathrm{C}$ may be reduced if the tip speed is decreased. However, at high Mach number, decreasing the tip speed is effective in reducing only the rotational noise and the blade slap. Vortex noise decreases at the slowest rate, so even at low tip speeds it remains as the dominant rotor noise source. This noise can be decreased by reducing the blade loading (i.e. $T / A_{b}$ ). On the other hand, rotational noise and blade slap can be decreased by lessening the disk loading (i.e. $T / A$ ). In order to decrease the blades tip speed, the rotors rotational speed may be decreased. This in turn decreases the frequency range of the rotor noise and is beneficial for large rotors. If the number of blades is increased, the magnitude of the rotational noises harmonics decreases and the fundamental frequency increases.

Decreasing the higher harmonic content of the rotors' airloads can decrease the rotational noise. In particular, this involves decreasing the BVI loads and hence certain types of blade slap. Further, the parameters that influence the rotor noise (i.e. tip speed, disk loading and the number of blades) are also major factors that govern the performance of the rotor. Therefore, the aerodynamic efficiency of the rotor can be improved by designing a quieter rotor, while maintaining the current level of performance and cost. The blade tip planform and section can change the rotor noise by altering the tip aerodynamic loads and the structure of the tip vortex. The airfoil section shape and thickness ratio at the tip must be selected so as to provide sufficient aerodynamic and performance characteristics at high Mach numbers. Compressibility effects are important factors in both rotational noise and blade slap production. So, an 
appropriate planform shape can maximize the tip vortex roll up and thus decrease the BVI loads. With that in mind, many blade tips (oger, vanes, etc...) or special devices (spoilers, subwings, end-plates, et...) have been designed to diffuse the tip vortex and modify its structure in order to decrease the BVI noise. These include passive designs such as blade tip sweep and unequal blade spacing have been proposed to decouple (or unphase) rotor noise sources (Baeder, 1997; and Sullivan et al., 2002). However, these attempts have not been successful without severely penalizing the performance of the rotor.

Due to such repercussions in the rotor's performance from passive systems or altering the mechanical operation of the rotor, numerous active technologies have been proposed. One example of an active technology which has been proposed involves employing positive mass blowing jets near the blade tip in order to alter the characteristics of the tip vortex (McAlister, 1996; and Yu, 2002). Many other active technologies have been investigated in BVI reduction. Such experiments focused on the modification of the near field tip vortex structure through the use of a tip air mass injection (TAMI) system. This TAMI system has been designed to decrease the noise output during BVI in low speed descending flight (White et al., 1975). Spanwise TAMI also decreases the drag from the induced velocity field introduced by the vortex. Another experiment involved the use of discrete blade tip jets. This experiment determined that the core of the tip vortex is less concentrated and that the coherence of the vortex strength and core near blade tip is reduced (Gowanlock and Matthewson, 1999). A recent experimental investigation modified the tip vortex core using a slotted blade tip which 
incorporated 4 channels distributed from the leading edge of the upper surface to the tip side edge. This produced a $60 \%$ decrease in the peak swirl velocity of the tip vortex (Mahalingam and Komerath, 1998; and Mahalingam, 1999).

Furthermore, the corresponding core radii for different wake ages were more than 3 times larger. These cause more rapid tip vortex diffusion for a slotted blade as a result of the enhanced mixing of the vortex core, which prevents the laminar region to sustain itself. The use of higher harmonic cyclic blade pitch (Brooks et al., 1991; and Yu et al., 1994) and active trailing edge flaps (Charles at al., 1994; and Dawson, 1995) have been proposed to rectify the unsteady blade airloads and reduce the intensity of the propagated BVI noise. These technologies provided some benefits on an actual rotor test, but these use open loop system, and thus require extensive mapping of conditions for significant noise reduction. These approaches reduced the rotor noise in certain flight conditions, but they also increased the vibration on the rotor. A further operational technology such as aircraft trajectory optimization or tip-path-plane AOA control have been postulated and have been found to offer benefits in changing the BVI miss distance at the rotor (Schmitz, 1998).

Validation requires careful prediction of rotor wake in order to understand relative changes in the wake for various flight conditions. As a result, a better understanding of the nature and focusing characteristics of the critical sound sources generated by the rotor is required. Another approach has been to relate far-field noise levels to source points on rotor (Sim et al., 1995; Lowson, 1996; and Strawn, 1997). Thus, it may be possible to modify aerodynamics at specific source points on the rotor thereby altering the 
propagated noise in a beneficial manner (although implementation of this idea is difficult). Lately, individual blade control (IBC) has received some attention from the research community because of the rapid development in smart structures technology (Wong, 2001; Coyne et al., 1997; Hardin and Lamkin, 1997; McAlister, 1996; White et al., 1975; Gowanlock and Matthewson, 1999; Yu, 2002; and Han and Leishman, 2003). Smart structures provide new-found design flexibility for advanced composite helicopter rotor blades. This allows the structure to sense and react as desired during different flight conditions to increase the rotor blade performance with respect to structural vibrations, acoustic radiation, and aeroelastic stability.

An example of an IBC system is integral actuation by using anisotropic actuators through the use of active fiber composites (AFC) with interdigitated electrodes. The base material characteristics and proof-of-concept model of this integral twisted actuated rotor blades have developed recently (Zdravkovich, 2003; and Norberg, 1985). The aim of this investigation was to design and analyze a beam-like structure, while taking into account the embedded anisotropic actuators distribution through the blade in a consistent manner so as to optimize the blade twist capabilities under the action of an external stimulus provided by the IBC control system. A two cell advanced composite rotor blade with integral anisotropic active plies was tested and designed in a wind tunnel.

Due to the aforementioned shortcomings and benefits found through the numerous techniques and investigations, a fundamental problem concerning BVI must be addressed by the research community. As a result, numerous numerical investigations related to BVI have also been performed and shall now be addressed. 


\subsubsection{Numerical Investigations in Blade (Airfoil)-Vortex Interaction}

In literature, the numerical treatment of a BVI event varies from aerodynamic model building concepts (Sim and Schmitz, 2000; and Berenger et al., 1997) to application of the thin layer Navier-Stokes (or Euler) equations (Srinivasan, 1986; Lee and Bershader, 1994; Wong et al., 2000; Oh et al., 2002; Lin and Chen. 1997; and Lee, 1994). The majority of numerical investigations invoked a Navier-Stokes based solver. Most investigations chose to embed a desired vortex velocity profile a distance upstream of the test rotor through superposition, before allowing it to convect downstream through the freestream.

Lee and Bershader (1994) showed that viscous effects play a significant role in parallel BVI using two different miss distances. Some researchers (Sim and Schmitz, 2000) used a global perspective to model BVI to investigate the resulting near and farfield acoustics. When coupled with the Kirchoff method, RANS simulations performed by Lee and Bershader (1994) have been able to achieve a similar global level of prediction. Using the full set of Euler equations in a CFD solver, Wong et al. (2000) demonstrated that the strongest acoustic signature occurs when the vortex impacted the lower surface of the blade. Yu (1996) investigated the effect of the vortex miss distance, $y_{v}$, using a comprehensive numerical code (CAMRAD-JA) coupled with a finite difference code (FPR). It was shown that changing the blade flapping deformation with a higher harmonic blade pitch control concept can effect $y_{v}$ (i.e. increase the missdistance), thereby reducing the BVI noise. Implementing neural networks into an active 
control mechanism has been investigated by Glabel et al. (2001) to reduce the noise and vibration generated during a BVI event. Open and closed loop direct and indirect neural control networks were used, some of which were capable of learned behavior using BVI data. Lee (1994) investigated the potential benefits of including a porous leading edge and found that it reduced the far-field noise by 20 to $30 \%$ during a BVI event. Vortex creation research has also been performed using either a starting vortex or a tip vortex. Figure 2.17 shows a numerical domain used by Mamou et al. (2001) for the study of AVI. In this investigation a shock tube was used for vortex creation by a heavily downward pitched airfoil upstream to generate a single coherent vortex through exploitation of the interaction with the oncoming shock wave.

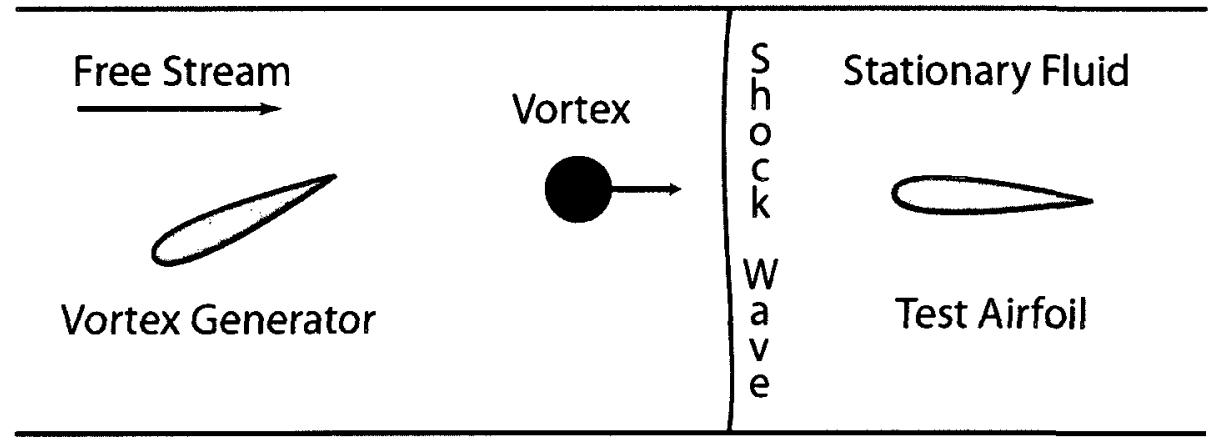

Figure 2.17: Schematic for the Numerical Shock Tube Employed by Mamou et al. (2001)

Numerical simulations of tip vortex creation, lifespan and behavior with age have been found to agree reasonably well with experimental results from Berenger et al. (1997). The two-dimensional BVI problem (i.e. AVI) has also been widely addressed using computational fluid dynamics (CFD) (McCorskey and Goorjian, 1983; McCorskey, 1985; McCorskey and Baeder, 1985; Singh and Baeder, 1996; and Lee and Baeder, 
2002). Through these investigations it was determined that as the freestream Mach number is increased, phase lag in the lift response grows.

After the airloads have been resolved, there are also high computational costs are associated with the evaluation of the acoustics. Therefore, $\mathrm{R} / \mathrm{C}$ noise prediction remains a challenging area of research (Brentner and Farassat, 2003). Due to the complicated wake structure on the $\mathrm{R} / \mathrm{C}$ 's rotor in forward flight, vortices may lie at many different orientations and inclinations with respect to the blades. It is not possible to model the aeroacoustics with reasonable computational cost or accuracy without the use of empirical predictions. Most aeroacoustic models give good noise prediction for idealized problems (i.e. and interaction of the blades with rectilinear type vortices). As a result, many comparisons have been of the idealized problem using a variety of different approaches (Caradonna et al., 1997; and Sim et al., 1997). Numerous researchers (Ringler et al., 1991; Sim et al., 1995; and Sim and George, 1996) have formulated the orientations and intersections of the acoustic lines to obtain a qualitative measure of the directivity of the dominant sound in the far-field produced by the BVI events on the rotor. Widnall (1971) developed a theoretical analysis of the impulsive sound generated by BVI. The analysis showed good agreement with experimental results for both wave form and the peak magnitude of the impulsive sound pressure.

The complexities of the unsteady nature of rotor flow pose a challenge for current CFD, especially in respect to capturing the wake. The current CFD capabilities are limited by grid resolution requirements and numerical dissipation of the scheme employed as a solution (i.e. the system of equations being solved). Both limitations have 
the tendency to generate non-physical dissipation of the rotor wake, which decreases the accuracy of the tip vortex as it ages. This problem has been overcome by Lee and Baeder (2002) who employed a chimera type overset grid. Alternatively, an adaptive grid would suffice as a solution to this issue. The chimera type grid employed used an O-type grid wrapped around the airfoil with two overset $\mathrm{H}$-grids that are moved relative to the O-grid, thus tracking the vortex as it convects past the airfoil. Employing this grid alongside an Euler CFD based approach. Despite high pressure gradients in the flow at or near a shock wave, the vortex emerged in a nearly undisturbed state; the initial radial symmetry of the vortex as maintained. Overset grids types provide afford finer resolution of the flow, which decrease numerical errors in regions of strong velocity or pressure gradients around the vortex, thereby decreasing the artificial numerical diffusion of the vortex during the interaction with the blade or airfoil. Ilie (2008) employed Large Eddy Simulation (LES) in order to study a BVI event. Numerous parameters were varied throughout this study in order to realize their effect during a BVI event. These parameters include the miss distance, airfoils angle of attack and the viscous core radius of the vortex itself. This study not only analyzed the aerodynamics of the interaction but investigated the aeroacoustics as well through the sound pressure level. Furthermore aeroelasticity was investigated for the interaction of a vortex with a flat plate. Wong (2003) employed a numerical method using Volterra integrals to study the BVI event. This method was validated with a RANS based CFD solution.

Furthermore in some cases, such as near the blade tip, the flow may be transonic. This results in the BVI problem being inherently nonlinear. As a result, quantitative 
prediction of the airloads requires advanced nonlinear CFD methods for accurate prediction of the event. Uncertainties of experimental results create a difficult situation to surpass the shortcoming of turbulence models, or to impose more accurate boundary conditions in the CFD solver (Dacles-Mariani et al., 1999). The advantage of employing a CFD simulation over experimental observation is the ability to capture data in the entire surrounding flow. Consequently, the flow patters may be captured in any region of the flow, which presents a superior understanding of the evolution of a tip vortex. If an understanding of how a tip vortex is formed the performance of the $\mathrm{R} / \mathrm{C}$ may increase and the effect of the BVI may be reduced. Accurate prediction of a BVI over the entire rotor in a three-dimensional viscous flow field is the ultimate goal of CFD. High resolution is necessary; thus, a high computational cost is inferred. High cost is a problem, as threedimensional overset grids are currently limited by computer memory and speed.

Complete CFD solutions have been a recent focus on the modeling of aeroacoustic effects of BVI (Strawn, 1997). But, to date, complete first principles based CFD approaches are not yet practical for rotor aeroacoustics problems due to significant numerical dissipation and dispersion errors associated with the tip vortex itself. 


\section{Chapter 3: Computational Methods for Investigating Airfoil-Vortex Interactions in Turbulent Flows}

The numerical simulation of the airfoil-vortex interaction (AVI) presents significant challenges. Most techniques either tend to alter the vortex's characteristics before the interaction, or are not able to capture the fluctuations of the aerodynamic coefficients. Two main solution techniques which aid in predicting unsteady aerodynamic responses of rotor blades during a blade-vortex interaction (BVI) exist within the scope of the current work. The first method uses either the Euler or Navier-Stokes equations to solve the BVI event as it occurs, and the second class is inviscid panel methods (Wagner et al., 2007).

The methods that employ either the Euler or Navier-Stokes equations are classified as computational fluid dynamics (CFD) methods. CFD uses discretized forms of either set of equation along with appropriate models to create a closed set of equations because these methods add more unknowns to the original set of equations. These methods consist of either the unsteady Reynolds-Averaged Navier-Stokes equations (URANS) or Large Eddy Simulation (LES). It is also possible to discretize and directly solve the Navier-Stokes equations; this method is referred to as Direct Numerical 
Simulation (DNS) (Piomelli, 2001). Although several solvers are commercially available, ANSYS CFX 11.0 will be used in the current work as it was readily available.

If a URANS approach is used in the CFD solver, a turbulence model is required. The use of a turbulence model has proven to be highly dissipative and may not explore the unsteadiness of the flow accurately as a result of the URANS requiring the turbulence model to model all relevant scales of turbulence. Several different form of turbulence models exist ranging from so called zero equation models, to one-equation models, as well as to two-equation models and finally second moment closure models. In the following, the term ' $n$-equation' model implies the addition of $n$ time-mean partial differential equations to the time mean RANS equations. Zero equation models simply add eddy-viscosity formulas algebraically to the system. One equation models add the time-mean equation of either eddy viscosity or the turbulence kinetic energy to the system, as well as algebraic formulas which model the various terms. Two equation models add turbulent kinetic energy and a second partial differential equation to the system as well as more algebraic modeling formulas (White, 2006). Second moment closures include transport equations, which include all physical processes which are present in a turbulent flow, for the Reynolds stresses (Tavoularis, 2008). These models could in theory account for non local and memory effects and could potentially be capable of predicting curved and rotating flows (Tavoularis, 2008). In practice, however, their success depends greatly on the relevance of the models for the various higher order terms on the accuracy of the numerical schemes employed by the solver for the solving of the nonlinear coupled differential equations (Tavoularis, 2008). Second order closures are 
often referred to as Reynolds stress models (Tavoularis, 2008). An alternative to modeling the Reynolds stress term, which results in the dissipation issue, is Large Eddy Simulation (LES). LES is computationally expensive however the characteristics of the vortex are preserved and instantaneous values of the flow variables are also obtained (Wagner et al., 2007). LES come at a significantly lower computational cost when compared to DNS, a fact that makes it an attractive alternative to other simulation methods for time-accurate unsteady solutions (Piomelli, 2001) of the AVI problem.

Direct Numerical Simulation (DNS) involves solving the Navier-Stokes equations directly, i.e. without modeling (White, 2006). It is restricted to low Reynolds number flows involving relatively simple geometries as a result of the wide range of length and time scales which are present in turbulent flows (Piomelli, 2001). The necessity of the tremendous resolution requirements for DNS of high Reynolds number flows of practical interest implies a tremendous computational power far beyond current supercomputing capabilities (Piomelli, 2001).

The panel method is another method that might be employed to solve the events which occur during the AVI. Panel methods require very little computational power and provide results with a reasonable degree of accuracy. The one downside to the panel method solution is that they are inviscid solutions and do not provide information on the viscous effects of the flow (White, 2006).

All relevant CFD approaches shall be addressed in the order presented above. The first of which shall be the RANS approach providing a discussion of two-equations model as well as a detailed section of the method which is used in the current work. 


\subsection{Reynolds Averaged Navier-Stokes (RANS) Simulation}

Several different models for the fluctuating terms have been developed (i.e. zero/one/two equation models) (White, 2006). For RANS simulations, the flow variables (velocity and pressure fields for incompressible flows) are divided into a statistically averaged (or mean) component and fluctuations. The mean components are solved for directly, while the fluctuations of the flow variables are modelled. Thus, RANS models all turbulent interactions within the flow field and DNS completely resolves these interactions. Prior to discussing the model employed in the current work, the governing equations for RANS must first be provided.

\subsubsection{Governing Equations for RANS}

The RANS equations are time-averaged equations of the motion for a particular turbulent fluid flow. In RANS, each component of velocity, $u_{i}$ is defined in terms of an averaged component, $\bar{u}_{i}=\frac{1}{\Delta t} \int_{t_{0}}^{t_{1}} u(t) d t$, and a fluctuating component, $u_{i}^{\prime}$ which mathematically can be surmised as $u_{i}=\bar{u}_{i}+u_{i}^{\prime}$ (Anderson, 2007). The RANS relations, which follow, provide an approximation based on knowledge of the properties of flow turbulence, giving approximate averaged solutions of the Navier-Stokes equations.

$$
\begin{gathered}
\frac{\partial \overline{u_{i}}}{\partial x_{i}}=0 \\
\frac{\partial \overline{u_{i}}}{\partial t}+\overline{u_{j}} \frac{\partial \overline{u_{i}}}{\partial x_{j}}=-\frac{1}{\rho} \frac{\partial \bar{P}}{\partial x_{i}}+\frac{1}{\rho} \frac{\partial}{\partial x_{j}}\left[\mu \frac{\partial \overline{u_{i}}}{\partial x_{j}}-\rho \overline{u_{i} u_{j}}\right]
\end{gathered}
$$

Notice that in Equation ( 3.2 ) the averaging process adds an extra term which must be resolved through turbulence modeling, as there is now an insufficient number 
equations to solve for all the unknowns which exist in the RANS system of equations (Anderson (2007)). The additional term in Equation ( 3.2 ) is often referred to as the Reynolds stress and is given as $-\rho \overrightarrow{u_{i} u_{j}}$. In order to close the RANS system of equations, a turbulence model must be employed. Several turbulence models are available such as the $k-\varepsilon, k-\omega$ or Reynolds stress equations. In the current work, the Shear Stress Transport (SST) model is employed prior to performing the Large Eddy Simulation on the flow field using ANSYS CFX 11.0, and this model shall now be outlined.

\subsubsection{Shear Stress Transport (SST) Model}

The shear stress transport (SST) model combines the existing $k-\varepsilon$ and $k$ - $\omega$ models using a blending procedure on modified equations (Menter, 1994), in order to determine the Reynolds stresses in Equation ( 3.1 ). This approach is used as the purpose of the SST is to maintain an accurate formulation of the $k-\varepsilon$ model in the near wall region while taking advantage of the freestream independence of the $k-\varepsilon$ model in the outer part of the boundary layer (Menter, 1994). Another reason for the use of this approach is that the $k-\omega$ model is too sensitive on the inlet boundary conditions specified for $\omega$, which is not an issue for the $k-\varepsilon$ model. As a result, the $k-\varepsilon$ model is generally preferable, however the $k-\omega$ model provides far superior accuracy in the sublayer of the boundary layer (Davidson, 2003) which makes it an attractive alternative when compared to more traditional turbulence models (i.e. the $k-\varepsilon$ or the $k-\omega$ models) for AVI investigations. The SST model blends the $k-\varepsilon$ or the $k-\omega$ models through the use of a blending function (for the theory behind SST refer to Section A.1 of Appendix A). This model 
imposes the $k-\omega$ model near the wall region and the $k-\varepsilon$ model in the regions away from the walls which are combined through the aforementioned blending function.

Upon performing the required SST analysis of the AVI, one may used the converged results to initialize the Large Eddy Simulation (LES), whose details shall now be discussed in full.

\subsection{Large-Eddy Simulation (LES)}

Large Eddy Simulation (LES) is an alternate numerical approach to that of RANS for the solution of the AVI problem. In terms of accuracy and the computational requirement, LES is an intermediary approach when compared to DNS and RANS problems (Piomelli, 2001). The main motivation for LES is that since the large energy carrying eddies are highly influenced by the boundary conditions, they can be resolved by computation. Whereas the small eddies (or unresolved scales) are modeled (Wagner et al., 2007). In general, the large scales of motion are more energetic than the small scales. As a result, their size and strength allow them to be effective transporters of the conserved properties (Piomelli, 2001). Therefore an approach which treats the large eddies with greater accuracy than the small eddies would be much more suitable. This conclusion leads to the use of the approach taken by LES (Wagner et al., 2007).

Large Eddy Simulations are three-dimensional and time dependent, but quite expensive albeit not nearly as costly as DNS (Wagner et al., 2007). LES is a much more viable option than DNS for either high Reynolds number flows or flows associated with complex geometry (Piomelli, 2001), which is discussed further in Section A.3 (refer to Appendix A). LES uses a space averaging operation which is applied to the Navier- 
Stokes equations. The mathematically closed form of this operation is found through a modeling technique which uses Fourier space (Piomelli, 2001). In principle, LES separates the large eddies, which are computationally resolved, from the small eddies which are modeled through this filtering process in Fourier space (Wagner et al., 2007). This process resolves (or captures) only the turbulent structures within a certain grid size while modeling all the smaller eddies (i.e. they are not captured by the filtering operation) (Piomelli, 2001). The advantage of this method is that it provides much more information than RANS while not requiring the computational time as DNS (Piomelli, 2001). LES is also powerful due to limitations presented by RANS (i.e. accuracy and capturing the turbulent structures) and DNS (computational power required) (Piomelli, 2001). The process involved with LES is as per the following:

1) A filtering operation must be defined to decompose the velocity, $u(x, t)$, into a summation of a filtered (or resolved) component, $\bar{u}(x, t)$, and a residual (subgrid scale (SGS)) component, $u^{\prime}(x, t)$. The filtered velocity field, $\vec{u}(x, t)$, is a three-dimensional, time dependent which represents the motion of the large eddies (Wagner et al., 2007).

2) The evolution of the filtered velocity field is derived through filtering the Navier-Stokes equations (Piomelli, 2001). These equations are similar to the unfiltered Navier-Stokes equations, however the filtered form now contains the residual stress tensor (SGS stress tensor), which is a result of the residual motions (Wagner et al., 2007). 
3) Closure of the system of equations is achieved through modeling the SGS stress tensor through the use of an eddy viscosity model (Piomelli, 2001)

4) The filtered equations are then solved numerically for, $\bar{u}(x, t)$, providing an approximation to the large scale motions in one sense of the turbulent flow (Wagner et al., 2007).

The details of the filtering process must first be defined, prior to presenting the filtered Navier-Stokes equations which govern LES.

\subsubsection{Generalities of the Filtering Process}

In order to define a precise way to compute the quantities in LES, it is necessary to define a velocity field which contains only the large scale components of the total flow field (Wagner et al., 2007). It has been shown that this may be done through a filtering process: the large scale is thus simulated and further represents the local average of the complete field (Leonard, 1974). A function, $f(x, t)$, can be defined such that this function contains all the scales (Leonard, 1974). If this function $f(x, t)$ represents an instantaneous variable of the flow field (i.e. velocity or pressure) and $G$ denotes an averaging Kernel (localized function) (Leonard, 1974), such that $G(0)>0, \int_{\Re^{d}} h(x) d x$ with $h(x) \rightarrow 0$ rapidly as $|x| \rightarrow \infty$.

The filter of $f$ be denoted by $\bar{f}$, which is defined as the convolution $f$ with a filter function, $G(x)$, then the filter mathematically is represented by

$$
\bar{f}(x)=\int_{\Omega} G(x-s) f(s) d s
$$


where $\Omega$ represents the flow volume, which is normalized such that $\int_{\Omega} G(s) f(s) d s=1$ as well as $G(-x)=G(x)$ (i.e. the filter is an even function) (Leonard, 1974). The fact that $G$ is an even function ensures that $\bar{f}=f$ when $f$ is constant (Leonard, 1974). The filtered Navier-Stokes equations may now be defined.

\subsubsection{Governing Equations for LES}

Before the filtered Navier-Stokes equations can be defined, it must be assumed that the error in the interchange of filtering and differentiation is negligible (i.e. $\partial \bar{f} / \partial x=\overline{\partial f} / \partial x$ ) (Wagner et al., 2007). Therefore, the filtered Navier-Stokes may be written as:

$$
\begin{gathered}
\frac{\partial \overline{u_{i}}}{\partial x_{i}}=0 \\
\frac{\partial \overline{u_{i}}}{\partial t}+\frac{\partial}{\partial x_{j}}\left(\overline{u_{i} u_{j}}\right)=-\frac{\partial \bar{P}}{\partial x_{i}}+\frac{1}{\operatorname{Re}} \frac{\partial^{2}}{\partial x_{j}^{2}}\left(\overline{u_{i}}\right)
\end{gathered}
$$

The application of the filtering process to the Navier-Stokes equations introduces two additional unknowns without any additional equations, thereby leaving an undetermined system (i.e. number of unknowns is larger than the number of equations) (Piomelli, 2001). This new system of equations now falls under the closure problem, for which LES can be shown by representing the velocity in terms of the mean/fluctuation as per RANS, through $u_{i}=\bar{u}_{i}+u_{i}^{\prime}$. The decomposition of the non-linear reactions may be written as $\overline{u u}=\overline{\overline{u u}}+\overline{\bar{u} u}+\bar{u} \bar{u}+\vec{u} \vec{u}$ (Piomelli, 2001). Note that $\bar{u}$ represents the large scale motions and $u^{\prime}$ represents the small scales (or subgrid scales (SGS)), of the total velocity field $u$ (Wagner et al., 2007). 
The filtered Navier-Stokes equations must be closed, which is done in LES through modeling the $\overline{u u}$ terms of $\bar{u}$ only (Wagner et al., 2007). Defining of the filtering process (see Equation ( 3.3 )), shows that generally, $\overline{\bar{u}} \neq \bar{u}$, unless $u$ is a constant or the filter is an ideal low pass filter (Wagner et al., 2007). Consequently, $\vec{u} \neq 0$ (Wagner et al., 2007). Note that in the RANS approach the decomposition of both the statistical mean and the fluctuations are achieved with a zero mean (Davidson, 2003). As a result, in LES, in order to overcome the closure problem, the filtered Navier-Stokes equations are rewritten in the following form

$$
\begin{gathered}
\frac{\partial \bar{u}_{i}}{\partial x_{i}}=0 \\
\frac{\partial \bar{u}_{i}}{\partial t}+\frac{\partial}{\partial x_{j}}\left(\overline{u_{i}} \cdot \overline{u_{j}}\right)=-\frac{\partial \bar{P}}{\partial x_{i}}-\frac{\partial \tau_{i j}}{\partial x_{j}}+\frac{1}{R e} \frac{\partial^{2}}{\partial x_{j}^{2}}\left(\overline{u_{i}}\right)
\end{gathered}
$$

where $\tau_{i j}=\overline{u_{i} u_{j}}-\overline{u_{i}} \cdot \overline{u_{j}}$ and is referred to as the subgrid scale Reynolds stress (Wagner et al., 2007). The SGS Reynolds stress is similar to the Reynolds stress introduced in RANS (Wagner et al., 2007). This similarity occurs because in both cases the unresolved scales, $u^{\prime}$ (the small eddies in LES and the turbulent fluctuations in RANS) result in stresses in the resolved scales of motion (the large eddies in LES and the mean velocity field in RANS) (Piomelli, 2001). In RANS, the velocity fluctuations, $u_{i}^{\prime}$, represent all the turbulent motions, whereas in LES, $u_{i}^{\prime}$ only represents the small scale motion (i.e. the subgrid scales) (Wagner et al., 2007). The implications of this are that the energy in the unresolved scales of LES represent a much smaller portion of the total energy of the flow with the energy in the unresolved scales of RANS (Wagner et al., 2007). Two main 
approaches exist to solve the LES closure problem, the first, which allows a filtering operator to be defined implicitly through the modeling of the SGS stress term, was found by Lilly-Deardroff (Wagner et al., 2007). The second is referred to as the Leonard approach. It uses a substitution of $u_{i}=\overline{u_{i}}+u_{i}^{\prime}$, into $\overline{u_{i} u_{j}}$ to result in $\overline{u_{i} u_{j}}=\overline{\overline{u_{i} u_{j}}}+$ $\overline{\overline{u_{i} u_{j}}}+\overrightarrow{u_{i}^{\prime} \overline{u_{j}}}+\overrightarrow{u_{i}^{\prime} u_{j}}$ (Wagner et al., 2007). Note that first term in the right hand side of the modified decomposition of the non-linear term, $\overline{\overline{u_{i} \bar{u}_{j}}}$, may be determined explicitly if the filter is defined clearly (Wagner et al., 2007). Using the above result in the SGS stress term in Equation ( 3.7 ), the following result may be obtained

$$
\tau_{i j}=\left(\overline{\overline{u_{i} u_{j}}}-\overline{u_{i}} \cdot \overline{u_{j}}\right)+\left(\overline{\overline{u_{i}}} \overline{u_{j}}+\overline{u_{i} \overline{u_{j}}}\right)+\overline{u_{i}^{\prime} u_{j}}
$$

This approach splits the SGS stress term into three terms which have different physical meaning in terms of the flow (Wagner et al., 2007). The first term, $\left(\overline{\overline{u_{i} u_{j}}}-\overline{u_{i}}\right.$. $\left.\overline{u_{j}}\right)$, represents the interaction of the large scales which produce effects in the small scales motion and is referred to as the Leonard stress (Wagner et al., 2007). The second term, $\left(\overline{\overline{u_{i}} u_{j}}+\overrightarrow{u_{i}} \overline{u_{j}}\right)$, represents the interaction of small eddies with the large eddies and is often referred to as the cross term (Wagner et al., 2007). Finally, the third term, $\overrightarrow{u_{i}} \overrightarrow{u_{j}}$, represents the transfer of energy from the small scales to the large scales and is referred to as the backscatter term (Wagner et al., 2007). The cross term may also produce backscatter since it concerns the interaction of the small scales with the large eddies as well (Wagner et al., 2007). In the Leonard approach, the filter must be explicitly known in order to determine the Leonard stress term, which is quite an inconvenience as in the 
grid based methods, the filter is often defined implicitly (Wagner et al., 2007). The issue of subgrid scale (SGS) modeling shall now be addressed.

\subsubsection{Subgrid Scale (SGS) Modeling}

As was mentioned in Sub-Section 3.2.2, applying the filtering process to the Navier-Stokes equations introduces a closure problem (Wagner et al., 2007). In order to solve this dilemma, the filtered Navier-Stokes equations were written in a form where the unresolved scales, represented by $\tau_{i j}$ in Equation ( 3.7 ), may be modeled (Wagner $e$ t al., 2007). In the current section, the two models which are employed with the highest frequency shall be discussed. These include the Smagorinsky model and the dynamic SGS model. The Smagorinsky model is the most commonly used model in LES to date (Wagner et al., 2007). This model is an eddy viscosity model, which is based on the increase in transport and dissipation which results from the effects of the SGS stress (Smagorinsky, 1963). It is this model which was used for the airfoil-vortex interaction analyses as it is a simple and common model which only models the scales of turbulence which are less than the grid size through a filtering process. The Smagorinsky model also makes use of the van Driest wall damping function (refer to Appendix A as Equation (A.22) Section A.2 for the associated theory).

Another, simpler alternative which requires much less computational power is an inviscid panel method which shall now be addressed. 


\subsection{Panel Methods}

Another method in obtaining a solution to the AVI problem is through the use of a panel method. The following discussion is presented as the LES AVI results shall be compared to the results obtained using the discrete vortex method by Renzoni (1987) in Chapter 5. The presentation of the information within the current section shall thus allow the reader to obtain some background regarding an inviscid panel method so that the differences present between the discrete vortex method AVI results and the LES AVI results become more evident. Currently, several panel methods are used, such as: the source panel method, the vortex panel method or a doublet panel method (Katz and Plotkin, 2006). These methods may be employed through the use of a constant source/vortex/doublet over a panel or these may be linearly varying (Anderson, 2007). Panel methods may be used to solve either two-dimensional or three-dimensional problems. Two-dimensional methods involve decomposing the geometry into a series of panels with end points defining the bounds of the panel and the midpoint in which the analysis is performed. Three-dimensional panel methods decompose the entire surface into a series of elements, from which an analysis is performed at the midpoint of the element which may then be used to extract the required information for the entire geometry (Katz and Plotkin, 2006). Panel methods may also be used in conjunction with methods that release a discrete amount of vortices in the flow, which shall be discussed in brief in Sub-Section 3.3.2.

Considering that the AVI problem is a two-dimensional problem which involves a vortex in the freestream impacting the airfoil, only the two-dimensional constant strength 
vortex panel method shall be presented in Sub-Section 3.3.1, as the alternate methods (including the three-dimensional methods) are considered beyond the scope of the current work. Another reason to use a vortex panel method is that the vortex method has proven to be the most accurate two-dimensional panel method (Katz and Plotkin, 2006). The source panel method is used for non-lifting analyses as a source that has no circulation; whereas, vortices have circulation which allows for the vortex panel method to be used for an analysis of a lifting body, as per the AVI problem (Anderson, 2007).

\subsubsection{Constant Strength Vortex Panel Method}

When limiting the analysis to incompressible (Mach number less than 0.3), inviscid flows (i.e. no viscous effects present), it is necessary to approximate the airfoils surface with a vortex sheet using a series of straight panels, as can be seen in Figure 3.1.

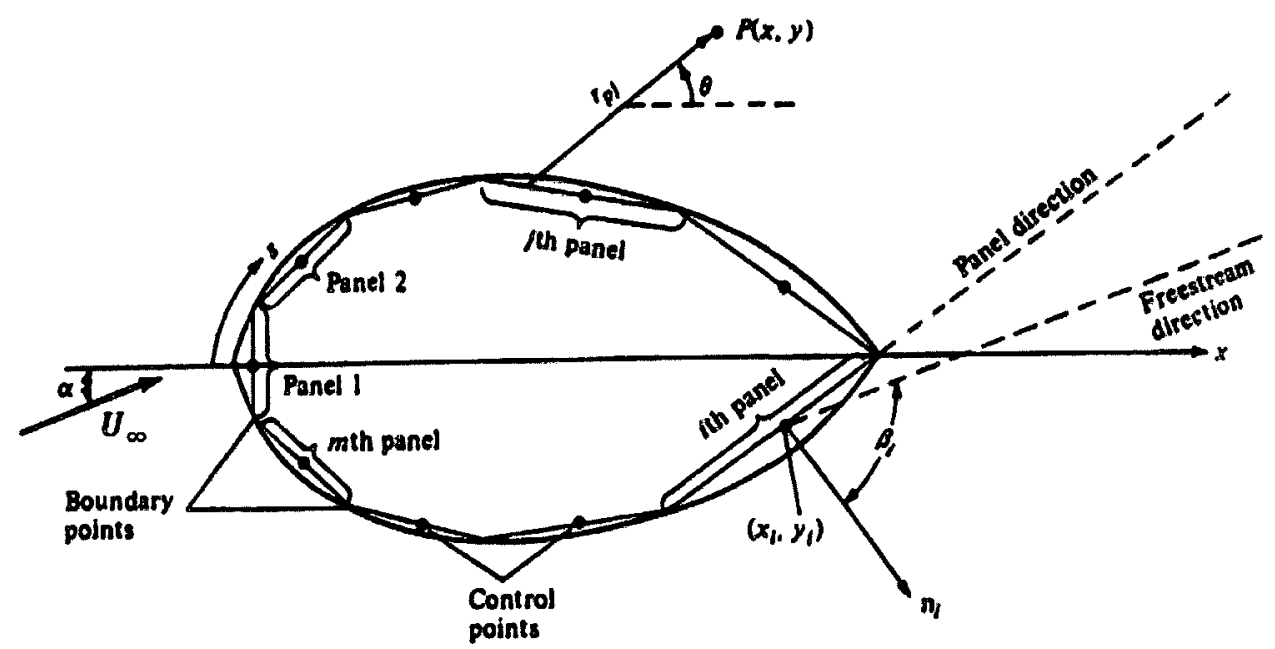

Figure 3.1: Airfoil Approximation for the Vortex Panel Method (Anderson, 2007)

The desired unknowns are the panel strengths, i.e. the strength of a vortex representing a panel. In other words, it is necessary to determine, $\gamma_{j}$ for $j=1$ to $N$ (where $N$ is the number of panels), such that the surface of the body becomes a streamline of the 
flow and ensures that the Kutta condition is satisfied (illustrated by Figure 3.2). The midpoint of each panel is referred to as a control point; it is at this point that the vortex strength is found to ensure that the normal component of flow velocity is zero, which is employed as a boundary condition. If $P$ is to represent any point $(x, y)$ in the flow domain, and $r_{P_{j}}$ is to represent the distance from any point on the j-th panel to the point $P$ in the domain (Anderson, 2007). The vector $r_{P_{j}}$ has an angle, $\theta_{P_{j}}$, with respect to the xaxis, which is given as

$$
\theta_{P_{j}}=\tan ^{-1}\left(\frac{y-y_{j}}{x-x_{j}}\right)
$$

The velocity potential induced at $P$ due to the vortex placed on the $\mathrm{j}$-th panel may be represented as

$$
\Delta \phi_{j}=-\frac{1}{2 \pi} \int_{j} \theta_{P_{j}} \gamma_{j} d s_{j}
$$

As the name of the method implies, the strength of the vortex, $\gamma_{j}$, on the $\mathrm{j}$-th panel is constant (Anderson, 2007). The potential at point $P$ due to the influence of all the panels is the summation of the potential at $P$ due to the j-th panel, i.e.

$$
\phi(P)=\sum_{\mathrm{j}=1}^{\mathrm{N}} \Delta \phi_{j}=-\sum_{\mathrm{j}=1}^{\mathrm{N}} \frac{\gamma_{j}}{2 \pi} \int_{j} \theta_{P_{j}} d s_{j}
$$

If the point $P$ is placed at the control point of the i-th panel, i.e. $\left(x_{i}, y_{i}\right)$, the angle of the vector between the $j$-th and $i-$ th panel, as well as the potential at the control point of the $i-$ th panel are given by 


$$
\begin{gathered}
\phi\left(x_{i}, y_{i}\right)=-\sum_{j=1}^{\mathrm{N}} \frac{\gamma_{j}}{2 \pi} \int_{j} \theta_{i j} d s_{j} \\
\theta_{i j}=\tan ^{-1}\left(\frac{y_{i}-y_{j}}{x_{i}-x_{j}}\right)
\end{gathered}
$$

Note that Equation ( 3.13 ) represents the contribution of all the panels to the potential at the control point of the i-th panel. As previously mentioned, the normal component of the velocity is to be zero at the control points (i.e. no flow is to enter the solid body). This normal component of velocity is the superposition of the contribution from the uniform flow velocity and the velocity induced by all the vortex panels (Anderson, 2007). The component of the freestream normal to the i-th control point is,

$$
U_{\infty_{n}}=U_{\infty} \cos \left(\beta_{i}\right)
$$

The normal component of the velocity which is induced at the $\mathrm{i}$-th control point by all other vortex panels is given as,

$$
U_{n}=\frac{\partial}{\partial n_{i}}\left[\phi\left(x_{i}, y_{i}\right)\right]=-\sum_{j=1}^{N} \frac{\gamma_{j}}{2 \pi} \int_{j} \frac{\partial \theta_{i j}}{\partial n_{i}} d s_{j}
$$

Application of the boundary condition leads to

$$
U_{\infty_{n}}+U_{n}=U_{\infty} \cos \left(\beta_{i}\right)-\sum_{j=1}^{\mathrm{N}} \frac{\gamma_{j}}{2 \pi} \int_{j} \frac{\partial \theta_{i j}}{\partial n_{i}} d s_{j}=0
$$

Equation ( 3.16 ) yields a linear system with $N$ equations and $N$ unknowns (Anderson, 2007). However, the Kutta condition must also be satisfied. Considering the trailing edge, as shown in Figure 3.2, the two panels at the trailing edge (i.e. the i-th panel and the (i-1)th panel) are very small. 
The Kutta condition insists that the circulation at the trailing edge is zero, i.e. $\gamma(T E)=0$, which requires that $\gamma_{i}=-\gamma_{i-1}$ (i.e. $\gamma_{1}=-\gamma_{N}$ in Figure 3.2) in order to negate the influence of the $\mathrm{i}$-th panel on the (i-1)-th panel (Anderson, 2007). This additional relation yields an over-determined system with $N$ unknowns, and $(N+1)$ equations (Anderson, 2007). As a result, one of the control points must be disregarded and the Kutta condition must be evaluated at that specific control point (Katz and Plotkin, 2006). The strength of each vortex panel, $\gamma_{j}$, may now be determined as the system is equal in $N$ equations and $N$ unknowns (Anderson, 2007). Once $\gamma_{j}$ has been determined, the tangential component of velocity may be determined through setting the velocity inside the airfoil to zero, as is illustrated in Figure 3.3.

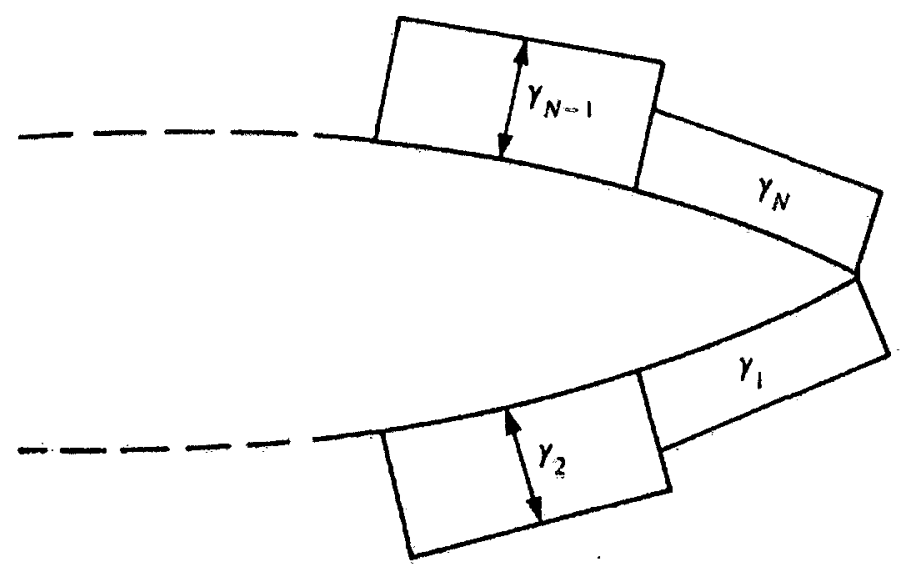

Figure 3.2: Panels of Constant Strength Vortices Near the Trailing Edge of the Airfoil (Katz and Plotkin, 2006)

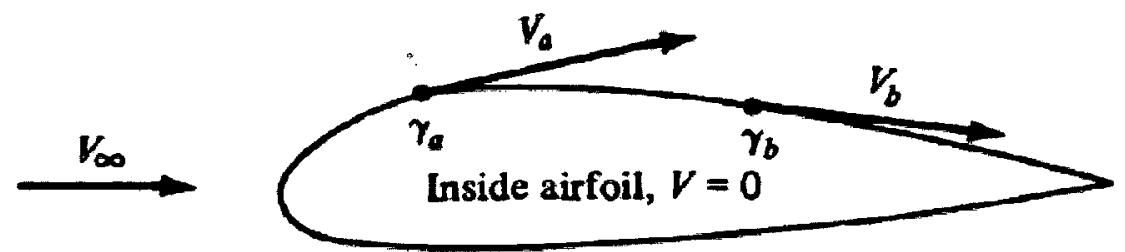

Figure 3.3: Condition Allowing the Tangential Velocity to be Determined, adapted from Anderson (2007) 
It can be shown that the local velocities tangential to the airfoil surface are equal to the local values of gamma (Anderson, 2007). Consequently, the resulting total circulation may be determined through:

$$
\Gamma=\sum_{j=1}^{N} \gamma_{j} s_{j}=0
$$

where $s_{j}$ represents the length of the $\mathrm{j}$-th panel. Similarly, the lift per unit span may be determined through the Kutta-Joukowski relation (Katz and Plotkin, 2006), i.e.

$$
L^{\prime}=\rho_{\infty} U_{\infty} \sum_{j=1}^{N} \gamma_{j} s_{j}=\rho_{\infty} U_{\infty} \Gamma
$$

It is worth noting that the results of this method are sensitive to the number of panels used, including the sizes and distribution of the panels (i.e. whether they are clustered around the leading/trailing edges) (Katz and Plotkin, 2006). Moreover, a degree of arbitrariness is introduced by the necessity of ignoring one of the control points (Anderson, 2007). What has been presented is the use of an analysis of an inviscid and incompressible nature to examine a viscous flow situation. Discrete vortices may be injected at arbitrary points in the domain to resolve the wake as well as some viscous interactions (Katz and Plotkin, 2006).

\subsubsection{Discrete Vortex Method}

Unlike the aforementioned panel methods, these methods employ a panel method in a time dependent sense (Fonseca et al., 2003). Both the addition of another panel in the wake (which is perpendicular to the trailing edge of the airfoil), and that of a further 
contribution of the wake to the potential of the flow are used to achieve this. This wake potential may be surmised as the contribution to $(M-1)$ point vortices previously generated (where $M$ defines the current time step). The second portion of the wake potential is a result of the amount of vorticity which is shed at time $M$ from the airfoils trailing edge. Correlations of this sort are modeled through the addition of the aforementioned additional panel. At time $(M+1)$, this panel is transformed into a discrete vortex with a strength equal to the contribution from the $M$ previous time steps. Also, it is necessary to add another boundary condition in which the circulation in the wake is equal to circulation around the airfoil with the opposite sign. This condition permits the amount of vorticity shed at the trailing edge to be determined. It thus provides a model for the generation of the wake's vortex. 


\section{Chapter 4: Blade Tip Vortices}

A wake of trailed and shed vortices is a direct result of lift-generation on a threedimensional lifting surface or unsteady flow. This wake vorticity in turn induces a velocity at the surface of the lifting body and thus influences the aerodynamic loading on the surface itself. The tip region in particular is enveloped by a region of high vorticity which rolls up fast into a dominant vortex. The roll up of tip vortex in terms of strength, velocity distribution/location defines the subsequent behavior of the lifting surface's wake.

In $\mathrm{R} / \mathrm{Cs}$, the rotor blades have a large pressure differential over the tip region, which causes tip vortices with high circulation and high swirl velocities as well as relatively small viscous cores. Since a rotor blade often passes very close to the tip vortices from the preceding blades, the vortex core plays a significant role in the wakeinduced velocity of a rotor and must be included in any representation of a wake's vorticity. As a result, the vortex core dimensions partly define the structure and evolution of tip vortices. Typically the core radius of a tip vortex is about $10 \%$ of the chord (i.e. approximately the chord thickness), although for rotary winged aircraft measurements of the core size are not extensive. The inboard shed and trailed vorticity is less important to the induced velocity field. As a result, it is typically represented by vortex sheets or 
neglected entirely during an investigation of the properties of the tip vortices or the bladevortex interaction. The tip of a rotor blade therefore induces significant velocities for several chord lengths downstream of the generating surface.

The rotor blade tip shape is affects both the strength and location of the tip vortices as they are trailed off a blade into the rotor's wake. Some tip shapes produce multiple vortices. These multiple vortices are stronger and dominate the flow in the rotors wake. Most R/Cs have rectangular or mildly tapered blade tips and a single tip vortex forming at the trailing edge of each blade tip. The overall roll-up of a tip vortex from a rectangular blade tip in terms of the vortex's strength and its initial position behind the blade is difficult to predict. Due to these issues tip vortex formation is a complex problem that involves high velocity with shear, flow separation, pressure equalization and turbulence generation. Each of these issues shall be addressed in some context in the following work.

In order to accurately model the tip vortex of a rotor blade, one must consider its variation with vortex age, the vortex core characteristics (such as radius and swirl velocity), and the vortex trajectory. There has been an exaggerated emphasis on vortex core parameters. Since identification of the vortex core parameters for a $\mathrm{R} / \mathrm{C}$ is currently not a well addressed issue, several models that incorporate a viscous vortex core including the simple Rankine vortex model exist (Spalart, 1998). The instant a vortex is formed, it has been found to be represented by a series of infinite line vortices, i.e. they are inviscid, and thus require no modeling of the core parameters, merely just the strength of the vortex itself. Most models, however, analyze the blade-vortex interaction problem 
and thus do not concern the tip vortex at the instant, as they have undergone vortex diffusion in some capacity. As a result the models are semi-empirical representations of the tip vortex characteristics. The required parameters for $\mathrm{R} / \mathrm{C}$ tip vortices are often interpreted or extrapolated from aircraft studies because they are not well documented for R/C tip vortices (Rorke et al., 1972; and Rorke and Moffit, 1977). Due to the mutual interaction between the vortices, the sustained proximity of tip vortices, and stretching of the filaments as they convect in a non-uniform flow, the validity of this approach is questionable. Tip vortex models used in rotor wake simulations usually are discussed in terms of a two-dimensional tangential (or swirl) velocity profile. Since other velocity components (i.e. axial/radial) are small and typically neglected in most applications. This is not justifiable for early wake ages. As mentioned, one characteristic parameter often used to describe tip vortices is the circumferential (or swirl/tangential) velocity.

Furthermore, in the past vortex flows have been considered axisymmetric due to a lack of a full three-dimensional representation. This opened a debate in the research community about the significant axial core velocity with respect to the swirl velocity (Mahalingam and Komerath, 1998). To investigate this dilemma, trailing vortices generated by fixed wing aircraft have been studied. Hence, it has been observed that the velocity profile with respect to the atmosphere may be directed towards the wing, thereby generating a wake-like profile. Or, it can be directed away from the wing, thus generating a jet-like profile. This study aimed to eliminate uncertainties associated with the influence of the axial velocity profile for a tip vortex. Other related studies provided measurements for a two bladed rotary wing aircraft in hover and have shown a strong 
wake like axial velocity distribution (McAlister, 1996). These results have been consistently confirmed in both hover and low speed flight (Martin and Leishman, 2002; Mahalingam and Komerath, 1998; Mahalingam, 1999; and Coyne et al., 1997).

The mechanism which describes the persistence of tip vortices for several rotor revolutions is still not clearly understood. The role of turbulence in the formation, evolution and dissipation of tip vortices has been examined as a potential solution to this problem (Dacles-Mariani et al., 1999). The experiment used flow pattern representations to show that the growth rate and persistence of tip vortices for several rotor revolutions are laminar processes. It was observed that the centrifugal force (i.e. the swirling motion) has a stabilizing effect and impedes the transport of turbulence energy from the small scales of turbulence to the core region (Schlichting, 1955). As a result, the core may be approximated as a solid-body like rotating region in which laminar flow is mainly present while dissipation occurs at the molecular level. The complexity of a three-dimensional flow as well as the significance and source of turbulence are therefore fundamental areas of research for both fixed and rotary wing aircraft.

The parameters and the mathematical description of the tip vortex at the instant it is formed (i.e. when it is a fully inviscid vortex) shall be addressed primarily. This will be followed by a commonly used model in order to represent a tip vortex while the bladevortex interaction may be occur, i.e. a viscous vortex model. The mechanism by which the inviscid vortex becomes a viscous vortex, i.e. vortex diffusion, shall then be presented. Finally, the structure of a tip vortex in terms of its transition from laminar flow at the core to turbulence outside the core shall then be addressed. Prior to providing 
information on the tip vortices (i.e. the velocity and static pressure distribution), some basic aerodynamic definitions related to tip vortices shall be provided.

\subsection{Aerodynamic Definitions Related to Blade Tip Vortices}

Some basic aerodynamic theory must first be discussed in order to present the required theory pertaining to inviscid vortices, viscous vortices, and vortex diffusion. Vorticity and circulation define the shape of the velocity profile as well as the strength of the tip vortex. Using these building block parameters, tip vortices may be described from the instant they are formed (inviscid vortices) until they actually interact with the next blade (viscous vortices), where the transition between the two states occurs as a result of vortex diffusion. The concept of vorticity shall now be presented.

\subsubsection{Vorticity}

Vorticity is essential to comprehend the entire workings of a blade-vortex interaction. As it allows one to describe a tip vortices velocity and pressure profile. To present the theory of vorticity an infinitesimal fluid element must be considered. This fluid element is permitted to translate along a streamline. It may also undergo some rotation and distortion. The amount of rotation and distortion of this fluid element depends on the velocity field to which the element is exposed. It can be shown through an analysis of the strain rates, distortion, and rotation, that the fluid element has an angular velocity, $\dot{\vec{\theta}}$, of

$$
\dot{\vec{\theta}}=\frac{1}{2}\left[\left(\frac{\partial w}{\partial y}-\frac{\partial v}{\partial z}\right) \hat{\imath}+\left(\frac{\partial u}{\partial z}-\frac{\partial w}{\partial x}\right) \hat{\jmath}+\left(\frac{\partial v}{\partial x}-\frac{\partial u}{\partial y}\right) \hat{k}\right]
$$


where $u, v$ and $w$ represent the $x, y$ and $z$ components of velocity respectively. Finally the $\hat{\imath}, \hat{\jmath}$ and $\hat{k}$ terms represent unit vectors in the $x, y$ and $z$ directions respectively (Anderson, 2007). In aerodynamics, the vorticity, $\vec{\omega}$, of said fluid element is defined as twice the angular velocity of the element, i.e.

$$
\vec{\omega}=2 \dot{\vec{\theta}}
$$

Alternatively, the vorticity may be expressed as the curl of the velocity field (Anderson, 2007), i.e.

$$
\vec{\omega}=\vec{\nabla} \times \vec{v}
$$

where $\vec{v}$ represents the velocity field and is given by

$$
\overrightarrow{\mathrm{v}}=\mathrm{u} \hat{\imath}+v \hat{\jmath}+w \hat{k}
$$

and the mathematical operator, $\vec{\nabla}$, is defined as

$$
\vec{\nabla}=\frac{\partial}{\partial x} \hat{\imath}+\frac{\partial}{\partial y} \hat{\jmath}+\frac{\partial}{\partial z} \hat{k}
$$

Based on the definition provided in Equation ( 4.3 ), two important definitions regarding the nature of the flow may be obtained. Firstly, if $\vec{\omega} \neq 0$ at every point within the flow, it is called rotational and this implies that all fluid elements have some finite angular velocity. However, if $\vec{\omega}=0$ throughout the flow field, the flow is called irrotational thus implying that each fluid elements motion through space is purely translational (Anderson, 2007). Another important expression in any aerodynamic analysis is the circulation, which shall now be defined. 


\subsubsection{Circulation}

Circulation, $\Gamma$, is a kinematic property of any fluid flow which is dependent on the velocity field as well as the choice of a closed curve, C. In aerodynamics, the curve, $\mathrm{C}$, is commonly taken as a closed curve surrounding the airfoil to provide a measure of lift (Anderson, 2007).

The present analysis pertains to a situation in which a closed curve, C, is considered in a flow field, with velocity, $\vec{v}$, and of which the tangent to the curve at the point of velocity measurement is denoted by, $d \vec{s}$. Figure 4.1 depicts such a curve as well as the velocity field and the infinitesimal tangent vector (Anderson, 2007).

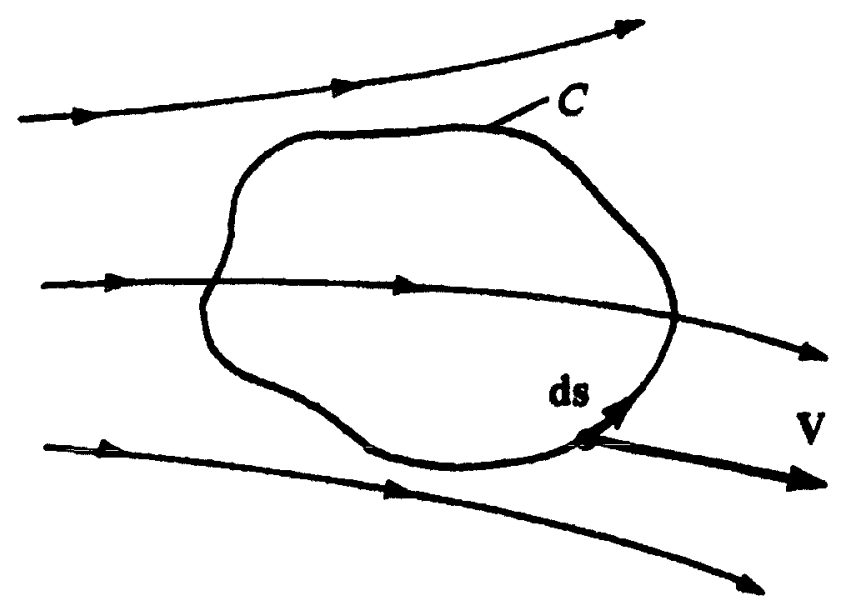

Figure 4.1: Depiction to Aid in Defining Circulation (Anderson, 2007)

The circulation, $\Gamma$, about the curve, $\mathrm{C}$, in Figure 4.1 is given as

$$
\Gamma=-\oint_{C} \overrightarrow{\mathbf{v}} \cdot d \overrightarrow{\mathbf{s}}
$$

Circulation may also be related to the vorticity of a flow field (Anderson, 2007). If the area, S, is bounded by curve, C, as illustrated in Figure 4.2, application of Stokes' theorem to Equation ( 4.6 ) yields 


$$
\Gamma=-\iint_{S}(\vec{\nabla} \times \vec{v}) d \vec{S}
$$

Applying Equation ( 4.3 ) to Equation ( 4.7 ) yields

$$
\Gamma=-\iint_{S} \vec{\omega} d \overrightarrow{\mathrm{S}}
$$

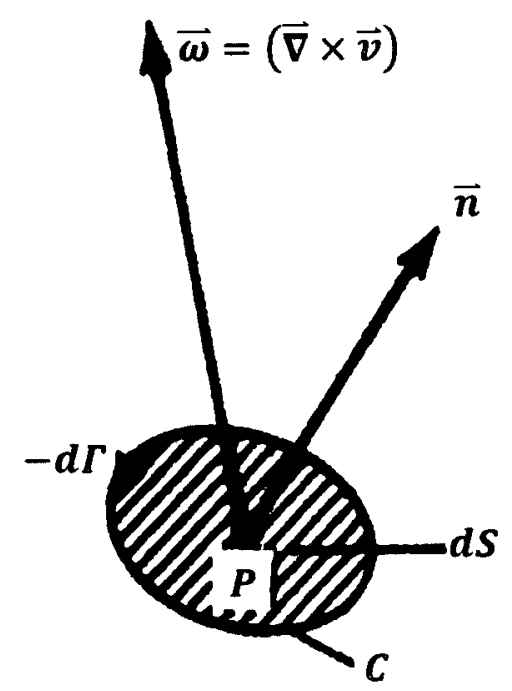

Figure 4.2: Relation Between Vorticity and Circulation (Anderson, 2007)

The circulation around any closed curve, $\mathrm{C}$, is also constant (Alekseenko et al., 2003), i.e.

$$
\frac{d \Gamma}{d t}=0
$$

Equation ( 4.9 ) states that the vorticity flux through a surface enclosed by a closed circuit, $\mathrm{C}$, is also must be constant (through the definition of vorticity as can be seen in Equation ( 4.3 )) which shall be employed in Section 4.4.

Using the definition of circulation, the tip vortex at the instant it is formed shall now be described through the use of potential flow theory. 


\subsection{Inviscid Blade Tip Vortices}

At the instant in which a tip vortex is formed, it may be represented as a straight line vortex of infinite extent and strength, $\Gamma$ (Johnson, 1980); also, the flow is assumed to be a vortex dominated flow (i.e. irrotational) which allows the use of potential theory to derive the velocity profile of the blade tip vortex. Consider a flow in which all streamlines are concentric circles about a point $P$, as illustrated in Figure 4.3 (Anderson, 2007).

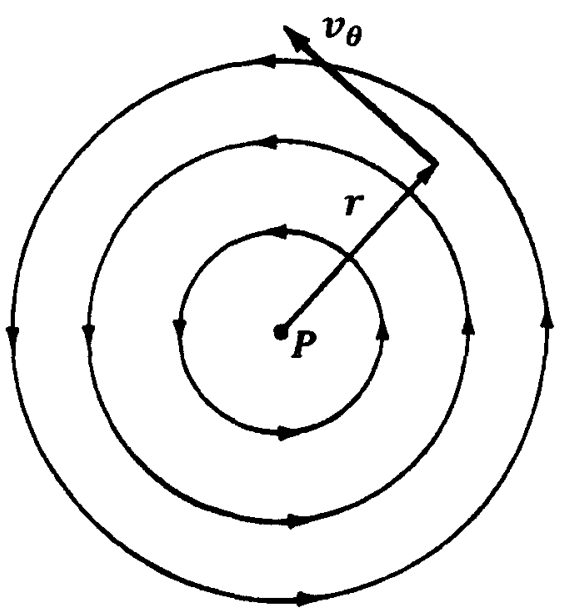

Figure 4.3: Illustration of a Vortex Flow (Anderson, 2007)

The velocity along any given circular streamline is constant, but it varies inversely from one streamline to another with the distance from the common center, i.e. point $\mathrm{P}$. Also, the velocity is presumed to not have any component in the radial direction, i.e., $v_{r}=0$ as has been reiterated through numerous studies concerning blade tip vortices. Furthermore, the tangential velocity is given through $v_{\theta}=C / r$ where $C$ is a constant and $r$ represents the radial distance (Anderson, 2007).

The constant, C, may be determined through evaluating Equation ( 4.6) around a streamline of given radius, $r$, which 


$$
\Gamma=-\oint_{C} \vec{v} \cdot d \vec{s}=-v_{\theta}(2 \pi r)
$$

Rearranging Equation ( 4.10 ) yields the tangential velocity, $v_{\theta}$, and the radial velocity, $v_{r}$, of an inviscid vortex as

$$
\begin{gathered}
v_{\theta}=-\frac{\Gamma}{2 \pi r} \\
v_{r}=0
\end{gathered}
$$

respectively (Anderson, 2007). This tip vortex induces velocities along the y-axis (which represents the rotor blade's position encountering a tip vortex from the preceding blade on the advancing side of the disk in forward flight). If the y-axis is viewed as the blade span, and the $x$-axis is normal to the blade (parallel to the freestream), the vortex induced upwash is

$$
v=\frac{-\Gamma}{2 \pi} \frac{x}{r^{2}}
$$

This upwash induces loading on the blade. The component parallel component of velocity which is induced on the blade is given as

$$
u=\frac{\Gamma}{2 \pi} \frac{y}{r^{2}}
$$

In Equation ( 4.13 ) and Equation ( 4.14 ), if $y_{v}=0$, which represents the vertical distance between the blade and vortex axes, then the velocity generated by the vortex is given as

$$
\vec{v}=\frac{\Gamma}{2 \pi y} \hat{\jmath}
$$


which is singular at $y=0$ (i.e. at the line vortex). As a result, for small $y_{v}$ and $y$, the vortex core must be included to obtain physically viable predictions of the induced velocity. Such is the case as the vortex progresses in age.

The circulation also represents the strength of the vortex flow, note that when $\Gamma>0$, the vortex rotates in the clockwise direction, which is reflected through the tangential velocity given by Equation ( 4.11 ) (Anderson, 2007).

As a result of theoretical vortices beginning as an inviscid phenomenon and noting that vortices in practical situations have a representative viscous core. Blade tip vortices with viscous cores shall now be detailed.

\subsection{Viscous Blade Tip Vortices}

As a tip vortex ages, it forms a viscous core through diffusion of the vortex (which shall be discussed in Section 4.4). Most models for tip vortices are semi-empirical representations of the tip vortex characteristics. These models generally include some representation of the strength of the vortex, $\Gamma$, as well as some representation for the viscous core which is generally provided by the core radius, $r_{c}$. The tip vortex core radius is typically assumed to be in the order of the airfoil thickness. Alternatively, Scully (1975) has suggested using $r_{c}=0.0025 R$ for most viscous vortex models. Another means of determining the core radius is by finding the location where within the core region the static pressure is half the total pressure drop experienced.

Tip vortex models usually employed in rotor wake simulations of twodimensional tangential (or swirl) velocity profile since other velocity components (i.e. 
axial/radial) are small and neglected in most applications (which is not justifiable for early wake ages). This is also consistent with observations that radial velocities are small for rotor blade tip vortices (Donsanijh et al., 1962).

Typical models for the velocity field of a viscous core tip vortex follow the configuration presented in Figure 4.4. Figure 4.4 illustrates the typical swirl distribution of a model for a viscous blade tip vortex, where the inner portion of the vortex (core region) is assumed to rotate as a solid body. The outer portion of the velocity distribution behaves almost as a potential flow. The viscous core radius, $r_{c}$, of the tip vortex separates the inner and outer portions of a viscous vortex is. This boundary also represents the radial location in which the swirl velocity, $v_{\theta}$, is a maximum.

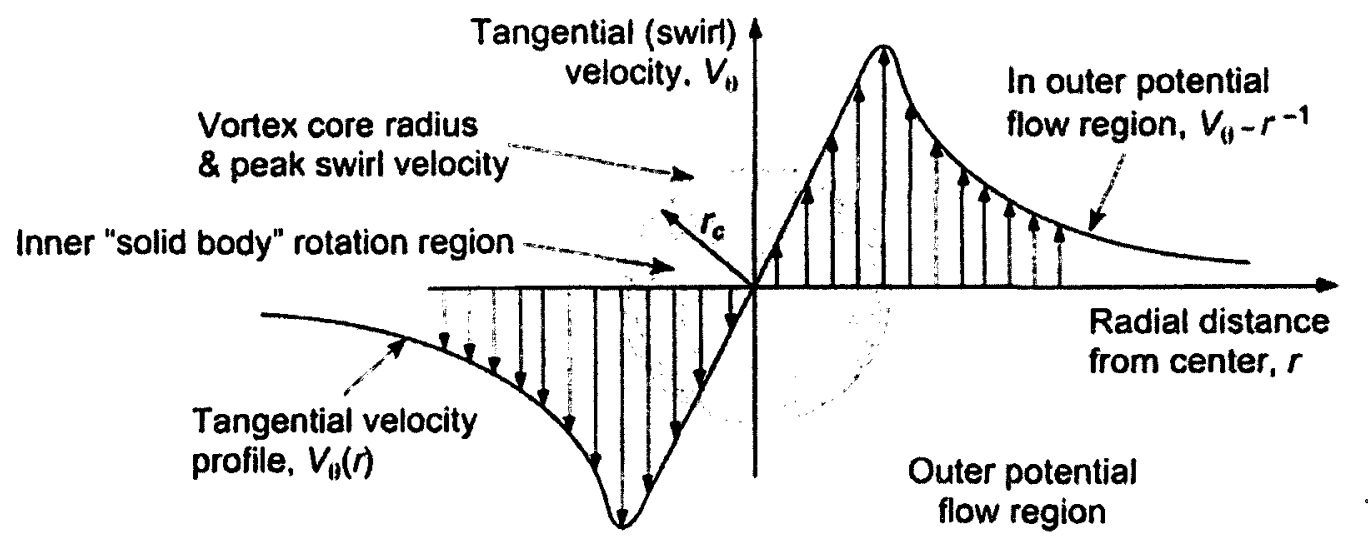

Figure 4.4: Typical Swirl Velocity Profile Inside a Tip Vortex (Leishman, 2006)

Types of viscous tip vortices range from models which have separate definitions for the inner and outer regions of the vortex to models which use exponential functions to effectively have a single definition which describes both the inner and outer regions. An example of a model of the former technique is the Rankine vortex (as is presented in Appendix B). An example of the latter is the Lamb-Oseen vortex. Most models of 
viscous tip vortices are comprised of laminar velocity profiles; however, turbulent models exist as well. The turbulent models normally only apply to cases where the diffusion of the tip vortex is considered and as a result shall be addressed only in Section 4.4. A variety of laminar models are presented in Appendix B, however the model which was deemed the superior model for representing R/C tip vortices, i.e. the Lamb-Oseen model, shall be presented. Further justification for the selection of the Lamb-Oseen model was given by Donsanijh et al. (1962) who showed that exponential velocity profiles provide a good correlation with tip vortex measurements for fixed wing generated tip vortices. In particular, axial velocities have shown good agreement and self-similarity of the LambOseen model as well as the Scully (1975) model (refer to Section B.1 in Appendix B) and that the fluctuations are present since small asymmetries are present within the tip vortex. Therefore, the Lamb-Oseen model shall be used to impose the $\mathrm{R} / \mathrm{C}$ tip vortex into the computational domain for the AVI investigation due to the ease of implementation in ANSYS CFX 11.0 when compared to the Scully (1975) model.

Generally, accounting for viscosity tends to smooth out the peculiarities present in the region of the vortex core in models of infinitely thin vortex filaments, as presented in Section 4.2.

\subsubsection{Lamb-Oseen Vortex}

A model which is often used to represent tip vortices generated by R/Cs was given by Oseen (1911) and Lamb (1932), who solved a simplified system of the NavierStokes equations in order to obtain a better approximation for the velocity profile 
produced by a rotor blade. This model is referred to as the Lamb-Oseen model the tangential velocity profile is given by

$$
v_{\theta}(r)=\frac{\Gamma}{2 \pi r}\left[1-e^{\left(-\alpha\left(r / r_{c}\right)^{2}\right)}\right]
$$

where, $\alpha=1.25643$. The solution obtained by Lamb and Oseen, i.e. Equation ( 4.16 ), is valid for a single viscous vortex in an unbounded, incompressible, laminar flow.

Several other tip vortex models employ exponential profiles for the swirl velocity distribution as well, these can be seen Appendix B. Numerous other investigations related to viscous tip vortices, some of which shall now be provided have been performed.

\subsubsection{Experimental and Numerical Investigations Related to Viscous Vortices and Tip Vortex Models}

The earliest measurements of $\mathrm{R} / \mathrm{C}$ tip vortices were performed with hot wire anemometry (HWA) (Simmons et al., 1966; Cook, 1972; Caradonna and Tung, 1981; and Tung et al., 1983). Measurements using HWA are limited however, by both the spatial resolution and the probe proximity concerns make measurements difficult at early wake ages (i.e. near blade tip).

As a result laser doppler velocimetry (LDV) was adopted to obtain more accurate measurements of a tip vortex's velocity profile. The nonintrusive nature of LDV makes it an attractive alternative to HWA measurements of R/C tip vortices since the relative dimensions of the probe, interference effects, and wire attrition are no longer concerns. The constraints associated with an LDV system for the measurement of $\mathrm{R} / \mathrm{C}$ tip vortices include optical access, the necessity of uniform flow seeding and good flow periodicity 
for statistical phase averaging as well as the requirement for coincident measurements on all 3 velocity components. The first uses of LDV concerning the aforementioned measurements were performed by Scully and Sullivan (1972), Sullivan (1973) and Landgrebe and Johnson (1974). A one-dimensional LDV measurement of rotor tip vortices was performed by Thomson et al. (1988) and Mahalingam and Komerath (1998). Two-dimensional LDV systems developed by Biggers and Orloff (1975) to investigate aspects of wake generated by 2 blades (see also Biggers et al., 1977(a, b)). Hoad (1983), Elliott et al. (1988) and Althoff et al. (1988) employed a two-dimensional LDV system with large stand-off distances in order to obtain tip vortex measurements. However, onedimensional and two-dimensional LDV systems tend to suffer from reduced spatial resolution as a result of elongated measurement values (especially with large stand-off distances). Thus neither are very suitable for measuring the small spatial scales and steep velocity gradients inside rotor blade tip vortices. A full three-dimensional phase resolved LDV systems is therefore necessary to obtain spatially accurate measurements in the rotor wake. If aligned accurately and operated in coincident mode (i.e. 3 velocity components measured required to be statistically correlated), these three-dimensional LDV systems offer superior spatial resolution. These three-dimensional systems have been used throughout investigations related to the rotor's wake and the blades' tip vortices. See Seelhurst et al. (1994, 1996), McAlister et al. (1995), Leishman et al. (1995), McAlister (1996), Han et al. (1997) and Martin et al. (2001) for examples of these investigations. Berenger et al. (1997) performed three-dimensional measurements of a tip vortex's evolution for an R/C using an LDV system to capture the creation of the 
tip vortex and its behavior after leaving the rotor blade. Measurements of the evolution of the tip vortices induced velocity field for several wake ages, as shown in Figure 4.5, were obtained by Bhagwat and Leishman (2002) using an LDV system.

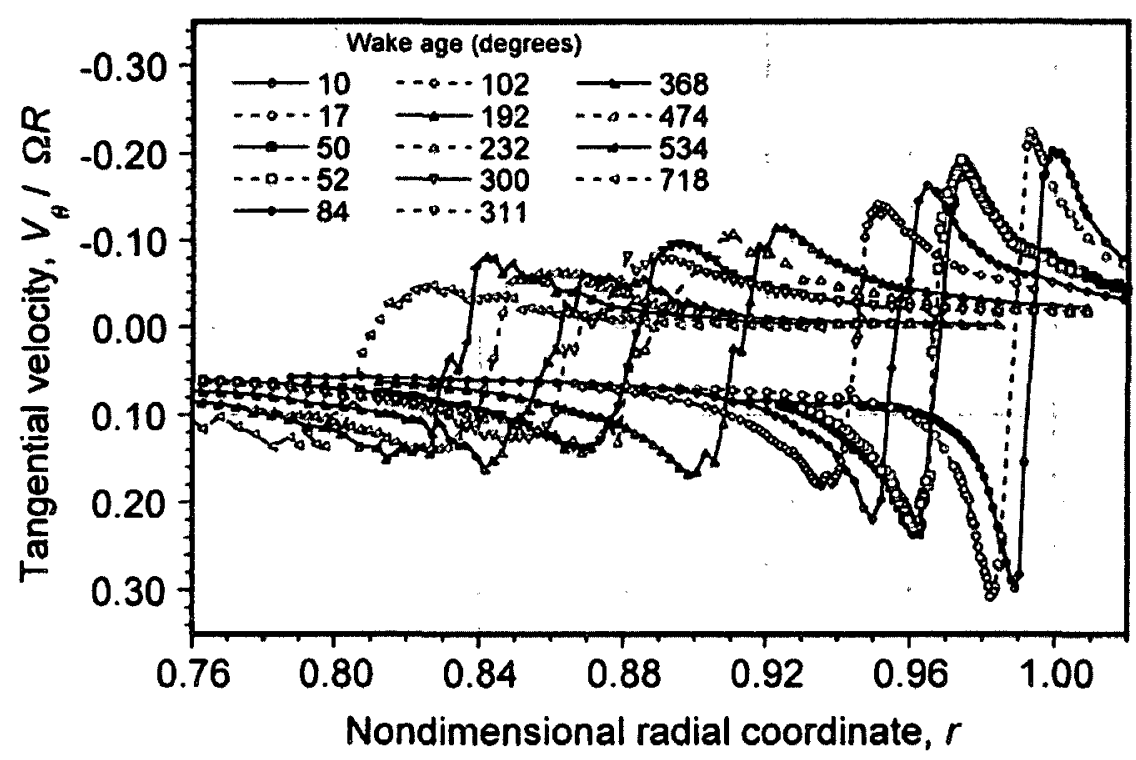

Figure 4.5: Measurements of the Swirl Velocity Profile of Tip Vortices at Various Wake Ages (Bhagwat and Leishman, 2002)

Notice in Figure 4.5 that as the age of the wake increases, the tip vortex convects both below the rotor and radially inward. Figure 4.5 depicts the basic characteristic of vortex diffusion. The vorticity contained in the vortex core diffuses radially outward with time; therefore the peak swirl velocities decrease.

Recently several particle image velocimetry (PIV) measurements have also been made concerning R/C tip vortices. For instance, Doolan et al. (1999) generated a threedimensional vortex to study its properties as it travelled downstream using a PIV system validating the method using HWA. Grant and Parkin (2000) measured the trailing tip vortices of wind turbine blades using PIV. Vortex meander was observed from these 
measurements. A method of selecting measurements based on the core location was used. This technique has also been used by Davenport et al. (1996), Horner et al. (1996) and Green et al. (2000). Malkiel et al. (1999) have also investigated using a two-dimensional PIV system, the accuracy of the embedded vortical structure in numerical simulations. Upon comparison, these results displayed sufficient agreement between the numerical model and the experimental actualization. However, the vortex release location is radially much further inward from vortex computational methods than flow visualization experiments. The complexity of this issue has been highlighted by Rule and Bliss (1998). McCorskey (1995) and Tang and Baeder (1999) have achieved greater success in the prediction of the origin point of a tip vortex using first principle finite difference results. Numerical diffusion and dispersion produce significant error in the subsequent vortex behavior (i.e. overprediction of the core growth) for many methods and are not currently accurate enough for design prediction.

That being said, the diffusion of blade tip vortices appears to be the current issue in terms of accurately modeling a blade-vortex interaction, and this topic shall now be addressed.

\subsection{Diffusion of Blade Tip Vortices}

Vortex diffusion is a consequence of the viscous effect, which, as assumed in Section 4.1 and Section 4.2, causes vortex lines to not coincide with fluid particle trajectories. This phenomenon is a direct result of Equation ( 4.9 ) and the no vorticity flux through a surface condition stated in Sub-Section 4.1.2 (Alekseenko et al., 2003). 
Vortex core dimensions define the structure and evolution of tip vortices. Most (but not all) of the vorticity in a tip vortex is concentrated in the viscous core region. The evolution of a tip vortex from a rotor blade's core of is illustrated in Figure 4.6. This figure also displays a rapid and asymptotic growth of the vortex core of a tip vortex generated by a rotor blade.

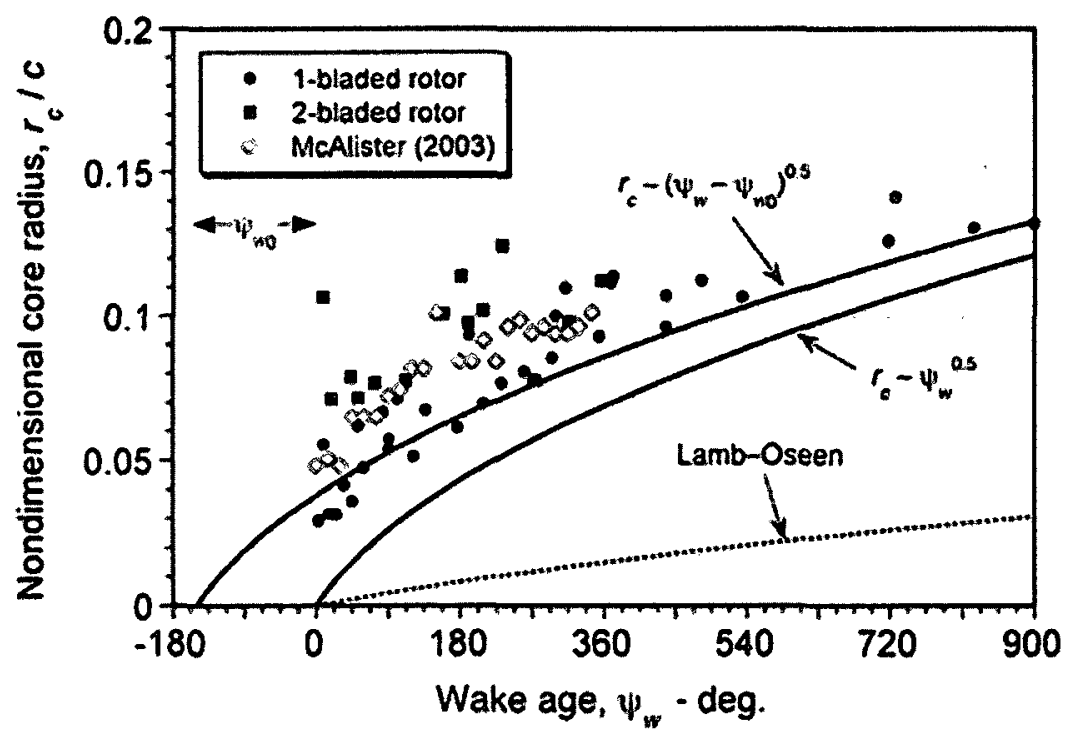

Figure 4.6: Viscous Core Radius Growth of Tip Vortices Trailed from Rotor Blades at Various Wake Ages (Leishman, 2006)

The close proximity passage of a rotor blade to a tip vortex (i.e. an oblique bladevortex interaction) even at $\psi_{b}=2 \pi / N_{b}$ (i.e. in hover) temporally alters the growth trend and a slight decrease in core size of the vortex occurs. This event fundamentally stretches the vortex, as becomes more evident for rotors with $N_{b}>2$ since the tip vortex does not convect very far through the flow and is substantially closer to the following blade. However after the blade passage, the viscous core continues to spread at a similar rate to prior to the blade passing the vortex. This presents difficulties in measuring vortex 
properties at older wake ages and is compounded by the flow's tendency to become aperiodic.

\subsubsection{Laminar Models of Tip Vortex Diffusion}

As per viscous tip vortices in steady state, (outlined in Section 4.3 as well as Appendix B), viscous diffusion of tip vortices may also be analyzed from either a laminar or turbulent perspective. A simple quantitative laminar model of vortex core radii growth with time based on Lamb's results has been addressed by several researchers (i.e. Lamb, 1932; Chigier and Corsiglia, 1971; and Duwag, 1993) and shall hence be presented.

Without external velocity gradients, which are responsible for vortex stretching and therefore effects the core development, the core radius varies with the wake age as

$$
r_{c}(t)=\sqrt{4 \alpha v t}
$$

The viscous effects for rectilinear vortex filament diffusion shall be illustrated. Initially, an infinitely thin vortex filament (i.e. an inviscid vortex) of circulation, $\Gamma$, is present in the axial direction (Alekseenko et al., 2003). This axisymmetric (imposed through the aforementioned conditions) vortex undergoes a time evolution governed by

$$
\frac{\partial \omega}{\partial t}=v\left(\frac{\partial^{2} \omega}{\partial r^{2}}+\frac{1}{r} \frac{\partial \omega}{\partial r}\right)
$$

It has been shown through applications of an appropriate set of boundary conditions to Equation ( 4.18 ) that the evolution of vorticity as a result of viscosity is given by

$$
\omega(r, t)=\frac{\Gamma}{4 \pi v t} e^{\left(-r^{2} / 4 v t\right)}
$$


Equation ( 4.19 ) shows that accounting for viscosity in the bulk of a fluids leads to vorticity diffusion. However, viscosity is in no way responsible for the generation of vorticity, as can be seen in Equation ( 4.19 ) (Alekseenko et al., 2003). Notice that if Equation ( 4.17 ) is substituted into Equation ( 4.19 ), the Lamb-Oseen profile given by Equation ( 4.16 ) is recovered.

Turbulent models of tip vortex diffusion shall now be detailed.

\subsubsection{Turbulent Models of Tip Vortex Diffusion}

The role of turbulence in the formation, evolution, and dissipation of tip vortices has been examined (Dacles-Mariani et al., 1999). The experiment used flow pattern representations to illustrate that the growth rate and persistence of tip vortices for several rotor revolutions are laminar processes. The centrifugal force (i.e. the swirling motion) has a stabilizing effect and impedes the transport of turbulence energy from the small scales of turbulence to the core region (Schlichting, 1955). Further studies have shown that as a result of turbulence generation, the vorticity diffusion within the vortex is much quicker than that given by Equation ( 4.19 ) (i.e. molecular diffusion). Therefore the tip vortex is expected to initially exhibit laminar behavior, thence the vortex progressively transitions to a more turbulent state. The tendency towards turbulence generation is related to

$$
R e_{v}=\frac{\Gamma}{v}
$$

among other factors, namely rotational stratification effects. 
The rate of development of the turbulent structure near the core alters the effective diffusion rate of vorticity from within the vortex. These effects can be incorporated into the model growth equation of the vortex core Equation (4.17) using an average turbulent viscosity coefficient, $\delta$, Equation ( 4.17 ), becomes

$$
r_{c}(t)=\sqrt{4 \alpha \delta v t}
$$

Research has shown that $\delta$ accounts for the more rapid core growth based on the average increase of vorticity diffusion due to turbulence generation. $\delta$ must therefore be deduced from vortex core structure measurements (Donsanjh et al., 1962; Squire, 1965; Ogawa, 1993; and Bhagwat and Leishman, 2000, 2002). Squire (1965) has shown that $\delta$ (i.e. the effective turbulent viscosity) is a function of the circulation strength of the trailed tip vortex, $\Gamma$. Since $\Gamma$ is conserved in practice and remains nominally constant as the vortex ages in the flow (Donsanjh et al., (1962); Mahaligan and Komerath, 1998; and Bhagwat and Leishman, 2000, 2002). Squire has also determined that it is reasonable to assume that $\delta$ remains constant throughout the simulation.

But, Bhagwat and Leishman (2002) have presented an empirical approach to obtain the $\delta$ parameter. An alternate approach was deduced by Iversen (1976). Iversen (1976) proposed the use of a non-dimensional swirl velocity and distance in order to determine the diffusion of vorticity due to turbulence. Iversen's correlations, expressed in terms of the wake age, are given as

$$
\left(\overline{v_{\theta}}\right)_{\max }=\left(\frac{\left(v_{\theta}\right)_{\max }}{\Omega R}\right)\left(\frac{\Omega R c}{\Gamma}\right)=\frac{\left(v_{\theta}\right)_{\max } c}{\Gamma}
$$




$$
\bar{d}=\left(\frac{\psi_{w} R}{c}\right)\left(\frac{\Gamma}{\Omega R c}\right)=\frac{\psi_{w} \Gamma}{\Omega c^{2}}
$$

Numerous tip vortex measurements for subscale $\mathrm{R} / \mathrm{C}$ rotors have been published by Leishman et al. (1995), Mahalingam and Komerath (1998) and McAlister et al. (1995). Further, Cook (1972) has made measurements for a tip vortex from a full-scale rotor. Based on these measurements, a plot of $\left(\overline{v_{\theta}}\right)_{\max }$ vs. $\bar{d}$ has been generated to determine the associated trends with the turbulent tip vortex. Figure 4.7 represents a cummulation of the aforementioned rotor measurements expressed in terms of Iversens correlations. Figure 4.7's decreasing trend of the swirl velocity confirms that the tip vortices diffuse logarithmically as the wake age increases, as per

$$
\begin{aligned}
\left(\overline{v_{\theta}}\right)_{\max }(\bar{d}+2.197)^{1 / 2} & =2.782 \text { for } \bar{d} \leq 50 \\
\left(\overline{v_{\theta}}\right)_{\max }(\bar{d})^{1 / 2} & =5.8 \text { for } \bar{d}>50
\end{aligned}
$$

Also, in Figure 4.7, the decreasing trend of the peak swirl velocity results from viscous diffusion, which may be seen through

$$
r_{c}=\sqrt{4 \alpha \delta \nu\left(\frac{\Gamma_{v}}{v}\right)}
$$

Equation ( 4.26 ) comes to fruition since $\psi_{w}=\Omega t$. 


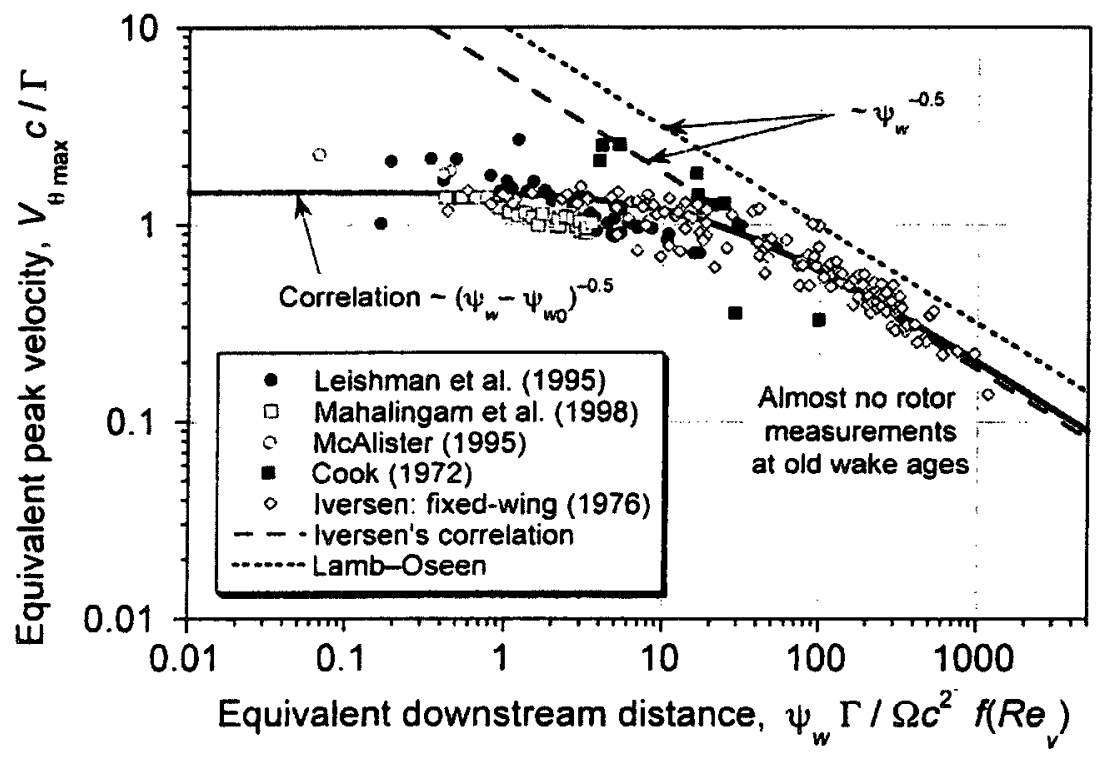

Figure 4.7: Correlation of Rotor Tip Vortex Measurements in Terms of Non-Dimensional Maximum Swirl Velocities and Downstream Distance (Leishman, 2006)

As a practical example, the Lamb-Oseen model shall be developed into a turbulent form. Using the Lamb-Oseen model it can be shown that

$$
\left(\overline{v_{\theta}}\right)_{\max } \bar{d}^{1 / 2}=\left(\frac{1-e^{-\alpha}}{4 \pi}\right) \sqrt{\frac{1}{\delta \alpha}\left(\frac{\Gamma}{v}\right)}
$$

Therefore, from Equation ( 4.27 ) it may be deduced that Iversen's correlation is equivalent to including an equivalent turbulent viscosity coefficient proportional to $R e_{v}$. As a result, Equation ( 4.26 ) may be rewritten as

$$
r_{c}\left(\psi_{w}\right)=\sqrt{4 \alpha \delta v\left(\frac{\left(\psi_{w}-\psi_{w_{o}}\right)}{\Omega}\right)}=\sqrt{r_{o}^{2}+4 \alpha \delta v\left(\frac{4 \alpha \delta v \psi_{w}}{\Omega}\right)}
$$

where, $\psi_{w_{o}}$ results in effective non-zero core radius, $r_{o}$, at the tip vortex origin where $\psi_{w}=0^{\circ}$ therefore providing a finite velocity at $\bar{d}=0$. In the Lamb-Oseen and Squire models, however, the swirl velocity is singular at the origin of the tip vortex and also has 
unrealistically high values of the swirl velocity at early wake ages. As a result, at large upstream distances, the velocity is inversely related to the $\sqrt{t}$ (i.e. $\left(\overline{v_{\theta}}\right)_{\max } \propto \psi_{w}^{-1 / 2}$ ) or equivalently the core radius increases with the $\sqrt{t}$ (i.e. $r_{c} \propto \psi_{w}^{1 / 2}$ ).

Several other investigations related to the diffusion of tip vortices have been performed. Some of these shall now be detailed.

\subsubsection{Further Investigations Related to Blade Tip Vortex Diffusion}

Numerous researchers have investigated diffusion of tip vortices in detail. For instance, Bossel $(1969,1971)$ employed a method of weighted residuals to compute rotationally symmetric vortex breakdown. He analyzed three types of tip vortices with a computational method and exponential functions as the weighted residuals. The three types of tip vortices examined include vortices generated in an initially uniform axial flow, vortices created at the leading edge of a blade, and trailing edge vortices. For each of these models, the initial profiles for both velocity and circulation of the vortex were provided. Furthermore, a critical swirl parameter was used to determine when vortex breakdown occurs. In another study, Bossel (1970) used weighted functions as well as the same computational method as previously mentioned to analyze boundary layers, vortices as well as axisymmetric wake/jet flows. Each study involved a series of exponential functions to the quasi-cylindrical Navier-Stokes relations to determine the evolution of the aforementioned flows. Based on such methods, Bossel (1973) also investigated the behavior of vortices with extremely high swirl profiles. This study focused on determining the breakdown of a vortex for a given swirl parameter for swirling flows 
with streamtubes of variable cross-section. Bossel (1968) developed stagnation criteria for vortices as well. From this, it becomes evident that various types of vortices have maximum associated swirl angles for breakdown of the core structure, which also have been shown to exhibit stagnation points at the axes of the vortices.

Other researchers, namely Spall and Gatski (1991), have performed fully threedimensional numerical simulations of vortex breakdown using the unsteady, incompressible Navier-Stokes relations. Four distinct types of breakdown were examined which include weak helical, double helix, spiral and bubble-type breakdowns. It was shown throughout this study that the asymmetric modes of breakdown are subjected to additional breakdowns as the vortex core evolves in the direction of the freestream. Furthermore, it was determined that the axial distribution of the freestream velocity has a significant effect on both the position as well as the type of vortex breakdown.

Based on the above discussion, it is apparent that if there exist models for both laminar and turbulent tip vortices, for both steady state analysis and vortex diffusion, there must be some structure of the tip vortex related to this. Refer to Appendix C for a discussion on the structure of the tip vortex. 


\section{Chapter 5: Aerodynamics of the Airfoil-Vortex Interaction I: Verification and Validation of the Computational Method}

In the present work, flows around an assumed two-dimensional flow around a NACA 0012 airfoil were simulated using Large Eddy Simulation. A vortex was superimposed into the domain in such a manner that the vortex axis is parallel to the blade span, as shown in Figure 1.1 and Figure 2.11. The present chapter seeks to validate the use of LES for AVI investigations through a comparison of experimental data obtained by Straus et al. (1990), furthermore the LES results shall be compared to the inviscid panel method results presented by Renzoni (1987).

Both grid and domain convergence have been performed and shall be discussed within the current chapter. Furthermore, the form of the Navier-Stokes equations used to solve for the aerodynamic coefficients during the blade-vortex interaction have been compared. The unsteady Reynolds-Averaged Navier-Stokes (URANS) equations using the shear stress transport (SST) models results were compared with the results obtained from applying the filtered Navier-Stokes equations which form the basis of large eddy simulation (LES). The LES equations employed the Smagorinsky model using the Van Driest model for the wall damping function. These comparisons were performed by imposing a vortex into the computational domain. Furthermore, URANS results for AVI 
were compared with LES results for AVI in order to compare a more traditional numerical method used for AVI investigations (URANS) with a new numerical method for AVI analyses (LES). This comparison was also performed to obtain an idea of how each of these numerical methods predict the aerodynamic coefficients (refer to Section 5.5 for the definitions of these coefficients) generated by AVI. The aerodynamic coefficients of the converged grid/domain/equation set shall be compared to the experimental data obtained by the aforementioned authors.

\subsection{Governing Equations and Computational Resources Employed}

To simulate the blade-vortex interaction, a finite-volume Navier-Stokes based solver was employed, namely ANSYS CFX 11.0. This solver was used for both the RANS/URANS and the LES simulations. For the solution of the filtered Navier-Stokes equations, i.e. the LES solution (see Equation ( 3.4 ) and Equation ( 3.5 )), a second order accurate conservative central-difference scheme was employed. For the RANS/URANS relations, a higher order accuracy implicit difference scheme was used for the spatial advancement. For the time advancement (for both URANS and LES simulations) a second order accurate backward explicit difference scheme was employed. For each approach, a trilinear interpolation technique was used to solve the velocity field. A linearlinear type interpolation was employed for the pressure field. The body forces were calculated through the application of a volume-weighted averaging technique. Also, for both RANS/URANS and LES, the root-mean-square (RMS) residual targets were set to $1 \times 10^{-12}$ for convergence to ensure that the required number of time steps (i.e. 95,000$)$ was reached. Three coefficient loops were performed at each time step to ensure sufficient 
resolution of the results at each time step. The numerical analysis was performed using a cluster of parallel computers with a total of 20 separate nodes.

The computational model employed including the initial fluid parameters as well as the geometry and mesh definitions involved shall now be discussed.

\subsection{Computational and Fluid Models}

For the validation and verification of the computational method which aims at acquiring the aerodynamic coefficients during a parallel blade-vortex interaction a quasi three-dimensional domain must be produced. A parallel blade-vortex interaction has been shown to be predominantly a two-dimensional phenomenon as discussed in Section 2.1. Therefore, the requirement of a slightly three-dimensional domain by ANSYS CFX 11.0 should not hinder the results obtained if the proper boundary conditions are used. The quasi three-dimensional geometry is achieved by using a thin three-dimensional computational domain is required by ANSYS CFX 11.0 in order to obtain a solution of the two-dimensional problem. On account of this requirement, the number of nodes within the xy plane of the domain to obtain a converged solution shall be examined. The thickness of the domain and the number of nodes in the third dimension shall also be analyzed with regards to convergence. The geometry was created using ANSYS ICEM CFD as it was readily available, and is illustrated in Figure 5.1.

In Figure 5.1, the airfoil length was selected from the study chosen for the validation of the method namely Straus et al. (1990). The details of this investigation shall be addressed in further detail in Sub-Section 5.4.1. The chord length of the airfoil is $c=0.46 \mathrm{~m}$. The profile of the airfoil (i.e. its shape) was selected as a NACA 0012. The 
fluid selected was air at $25^{\circ} \mathrm{C}$ with a reference pressure of $1 \mathrm{~atm}$. The computational domain which is based on the geometry presented in Figure 5.1 shall now be presented.
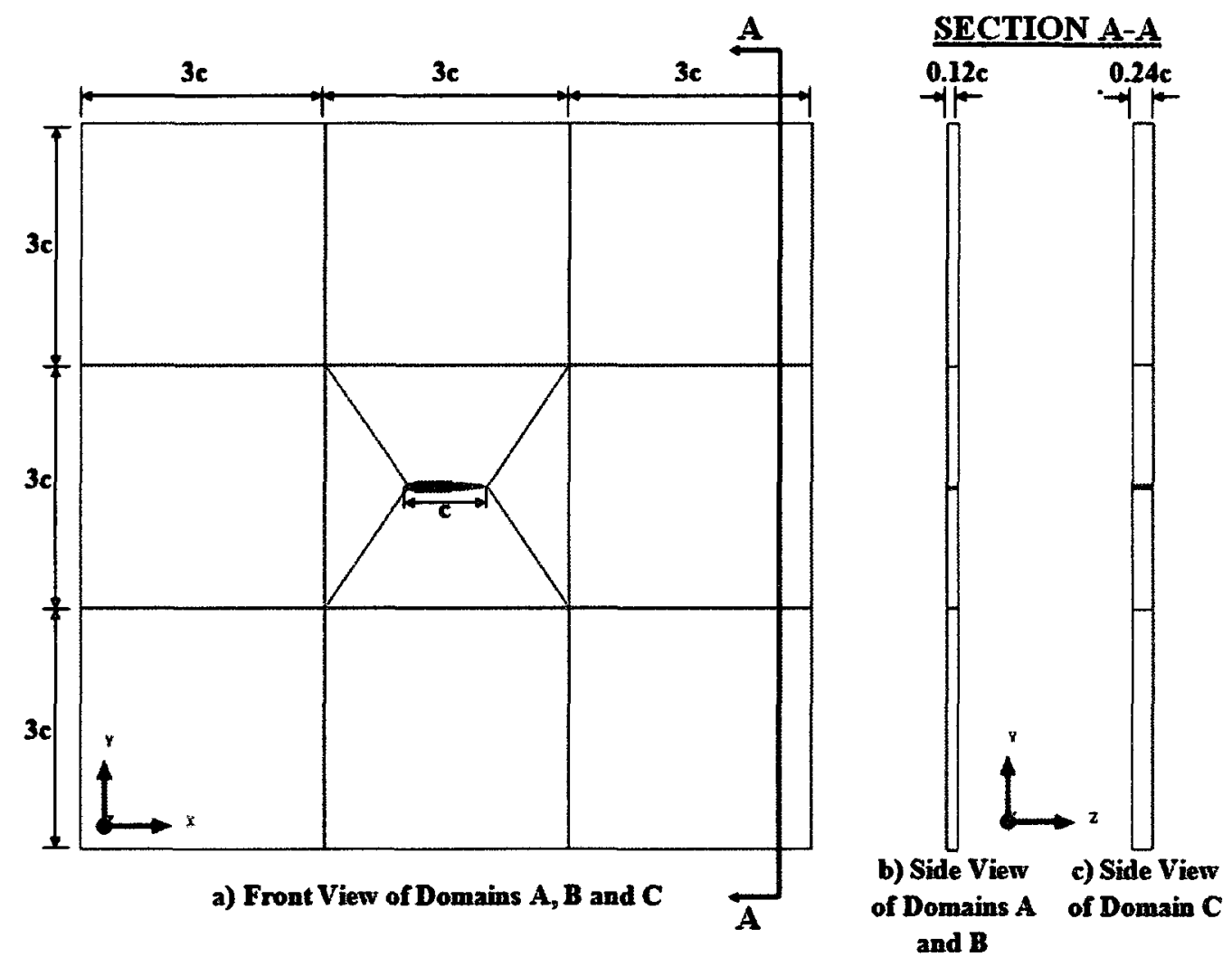

Figure 5.1: Geometry Created for the Validation of the Computational Method

\subsection{Computational Domains}

For reasons of computational efficiency, the domain based on Figure 5.1 was divided into 12 blocks, which are illustrated in Figure 5.2. Based on the block definition presented in Figure 5.2, the size of the structured computational grid may be defined. Figure 5.3 illustrates the definition of the meshes that will be used for the validation and verification analysis. 


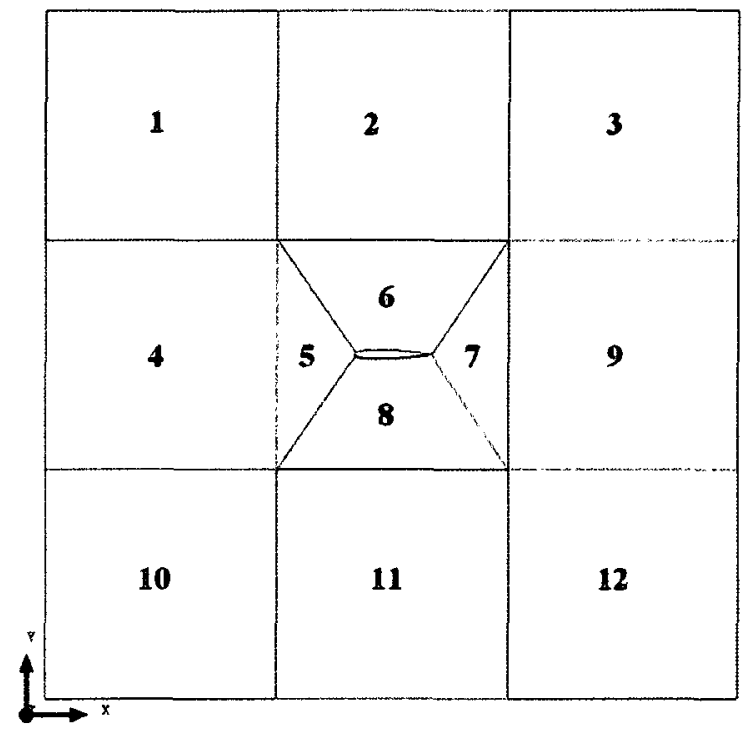

Figure 5.2: Block Definitions in ANSYS ICEM CFD

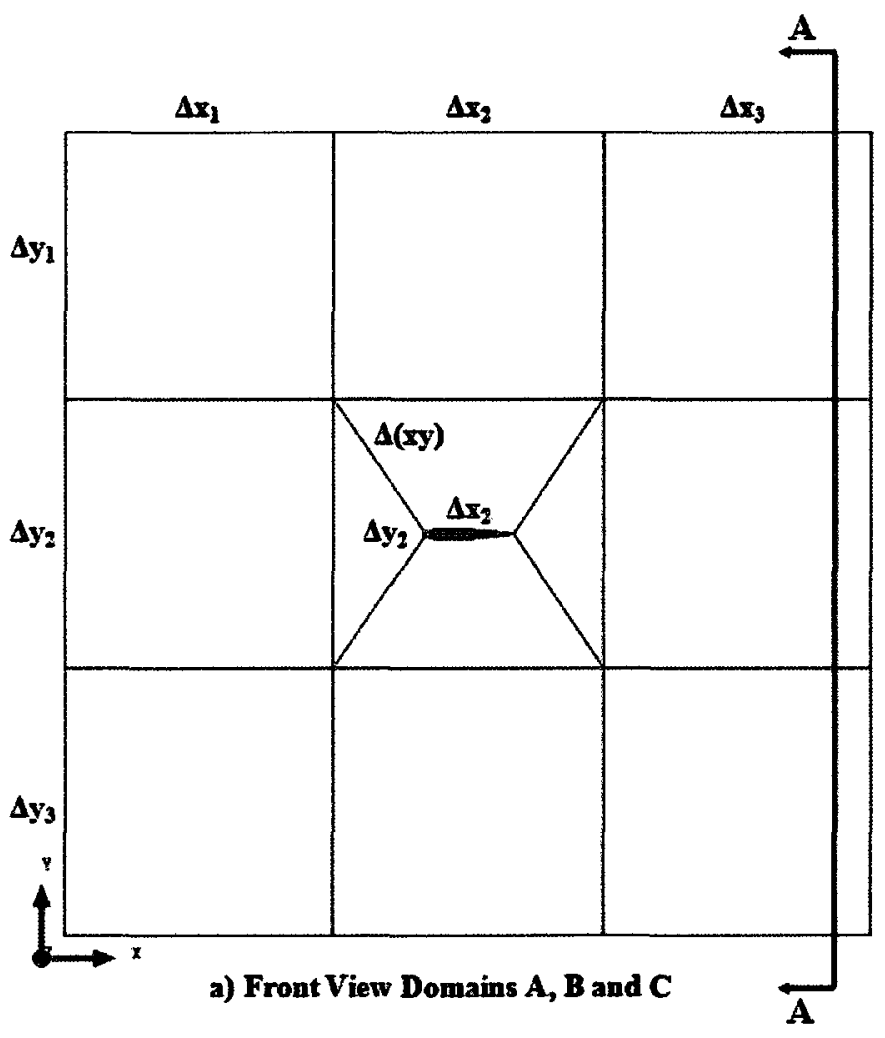

SECTIONA-A

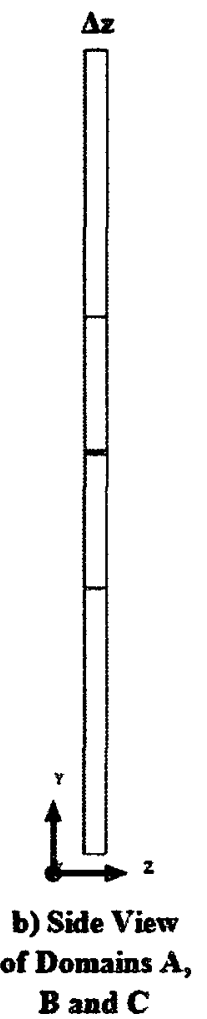

Figure 5.3: Node Definition for the Computational Domains Used in the Validation and Verification of the Computational Method 
Defining each of the $(\Delta x, \Delta y, \Delta z)_{i}$ parameters as well as $\Delta(x y)$ results in three separate computational domains which shall be referred to as Domains A, B and C. These parameters represent the number of nodes associated with each block defined in Figure 5.2. Table 5.1 represents the definitions of the number of nodes associated with the values defined in Figure 5.3 including the total number of nodes associated with each domain.

\begin{tabular}{|c||c|c|c|c|c|c|c|c||c|}
\hline Domain & $\Delta \mathrm{x}_{1}$ & $\Delta \mathrm{x}_{\mathbf{2}}$ & $\Delta \mathrm{x}_{\mathbf{3}}$ & $\Delta \mathrm{y}_{\mathbf{1}}$ & $\Delta \mathrm{y}_{\mathbf{2}}$ & $\Delta \mathrm{y}_{\mathbf{3}}$ & $\boldsymbol{\Delta}(\mathrm{xy})$ & $\Delta \mathrm{z}$ & Total \\
\hline A & 64 & 180 & 64 & 64 & 180 & 64 & 180 & 4 & 760,464 \\
\hline B & 64 & 250 & 100 & 64 & 250 & 64 & 250 & 4 & $1,365,648$ \\
\hline C & 64 & 180 & 64 & 64 & 180 & 64 & 180 & 8 & $1,520,928$ \\
\hline
\end{tabular}

Table 5.1: Node Definitions Resulting in the Compuational Domains Used for Validation

For $(\Delta x, \Delta y, \Delta z)_{i}$ a uniform spacing was employed in each computational domain. Whereas for $\Delta(x y)$, the nodes were clustered around the airfoil wall with an expansion ratio of 1.05. For the grid convergence analysis, Domains A and B shall be used. The domain convergence analysis compares, Domains A and C shall be compared. Finally, results for both URANS and LES shall be compared using the converged grid and domain for the verification study. The resulting multi-block structured grids for the airfoil centric computational domains are illustrated in Figure 5.4.

Note that for Domain B, the grid has a larger concentration of nodes in the blocks where the wake will occur, i.e. downstream of the airfoil. This is not shown since there is little noticeable difference in the images obtained. 


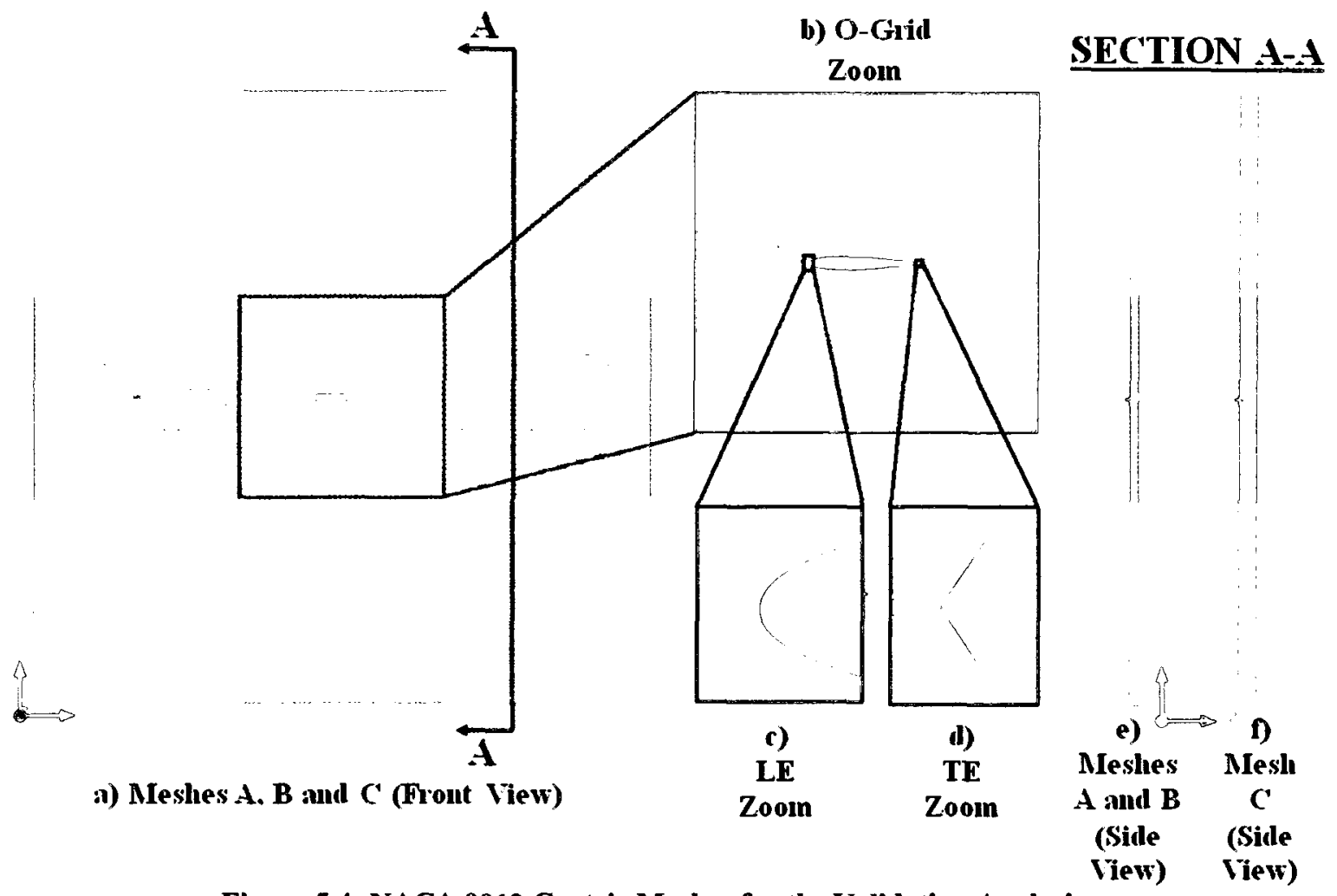

Figure 5.4: NACA 0012 Centric Meshes for the Validation Analysis

For each computational domain, a time step of $1 \times 10^{-6}$ seconds was used. This time step was selected to ensure sufficient accuracy with time. It is required as a result of the selection of an explicit time-marching scheme as well as the temporal resolution requirement of LES. The temporal resolution requirement for an LES simulation states that the Courant-Friedrichs-Lewy (CFL) number must be less than unity for sufficient temporal resolution. Throughout each domain and the entire simulation in the present analysis it was ensured that the CFL was below unity. The Smagorinsky constant was $C_{s}=0.1$ to ensure that no numerical dissipation of the vortex occurs as it traverses the computational domain. 
Upon creating the computational domain, one impose the necessary boundary conditions also the domain must be initialized in a proper manner in order to superimpose the vortex into the domain. These details show now be addressed.

\subsection{Boundary Conditions and Initialization}

The present analysis attempts to validate the use of LES for a parallel bladevortex interaction. As a result, the experimental work performed by Straus et al. (1990) was selected to validate the solution method. Therefore, to obtain the required parameters related to the boundaries and initialization of the domain, the work performed by Straus et al. (1990) must first be presented in further detail.

\subsubsection{Further Details of the Analysis Performed by Straus et al. (1990)}

Straus et al. (1990) conducted an experiment in a $(1.2 \mathrm{~m} \mathrm{x} 1.8 \mathrm{~m})$ closed circuit wind tunnel. A schematic of the experimental setup is presented in Figure 5.5.

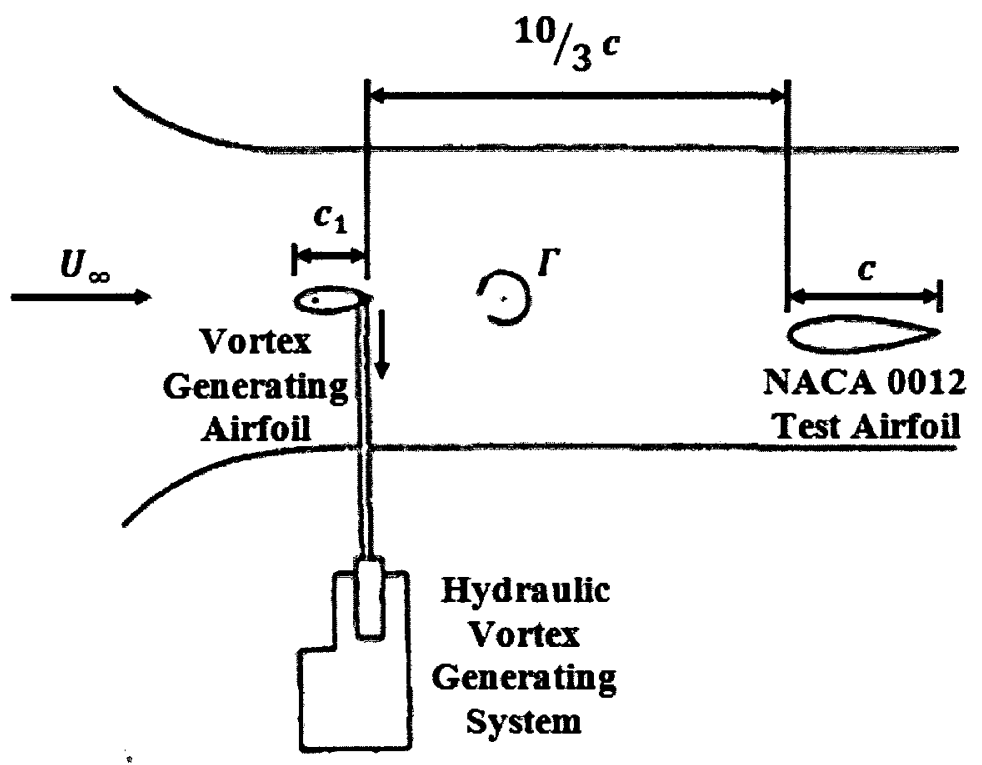

Figure 5.5: Experimental Setup Used by Straus et al. (1990) 
The test airfoil, i.e. the NACA 0012 , was placed approximately $150 \mathrm{~cm}$ downstream of the vortex generating airfoil (NACA 0018), as is illustrated in Figure 5.5. The dimensions of each airfoil used to conduct the experiment are given in Table 5.2 also included are the airfoil profiles which were employed.

\begin{tabular}{|c||c|c|c|}
\hline Airfoil & Profile & Chord [m] & Span [m] \\
\hline Vortex Generator & NACA 0018 & 0.25 & 1.52 \\
\hline Test & NACA 0012 & 0.46 & 1.80 \\
\hline
\end{tabular}

Table 5.2: Dimensions of the Airfoil Sections Used by Straus et al. (1990)

Table 5.3 provides the Reynolds number (based on the test sections chord length) and the freestream Mach numbers used by Straus et al. (1990) as well as the conditions which define the vortex (i.e. its strength, $\Gamma$, and viscous core radius, $r_{c}$ ). Also presented in Tab is the angle of attack, $\alpha$, of the test airfoil.

\begin{tabular}{|c|c|}
\hline$U_{\infty}[\mathrm{m} / \mathrm{s}]$ & 12.2 \\
\hline$\alpha[\mathrm{deg}]$ & 0 \\
\hline$\Gamma\left[\mathrm{m}^{2} / \mathrm{s}\right]$ & 0.89792 \\
\hline$r_{c}[\mathrm{~m}]$ & 0.02325 \\
\hline$R e[1]$ & 300,000 \\
\hline$M[1]$ & 0.036 \\
\hline
\end{tabular}

Table 5.3: Fluid Parameters Employed by Straus et al. (1990)

The test airfoil is fitted with 21 pressure taps positioned around its surface on one side and 3 pressure taps on the other side. The pressure taps are clustered about the leading edge since this is the location with the largest pressure gradient. Detailed measurements were obtained by inverting the airfoil and repeating the experiment for the same inflow parameters and pitch angle. The 24 taps are connected by 2 miniature air driven scanivalves to two high resolution differential pressure transducers. The 
transducers were equipped with a temperature compensation circuit and separate amplifiers with gains of approximately 10,000 . To decrease the response time of the pressure measurement system and increase the signal to noise ratio, all the electronics of the pressure measurement system were placed within the test airfoil, thereby providing a response time of $0.5 \mathrm{~ms}$. The test sections pressure measurements were referenced to the static pressure of the freestream. This was done by attaching a reference tap on each transducer to two small tanks inside the test airfoil of sufficient size to maintain a constant pressure throughout the interaction. These tanks were in turn connected to a static tap of the incidence flow Pitot-static tube.

When the upstream airfoil (the vortex generating airfoil) is suddenly pitched a single two-dimensional cast-off vortex is generated. This vortex then convects downstream parallel to the test airfoil. Velocity measurements were made in both the freestream and the boundary layer of the test airfoil using a hot-wire anemometry. The velocities profiles were obtained using a traverse mechanism attached to the upper wall of the wind tunnel. The unsteady component of the flow created by the vortex was measured by biasing the anemometers output using the steady mean flow voltage and amplifying it. The ensemble averaging was performed using a Nicolet digital oscilloscope triggered by the motion of the vortex generating airfoil. The data obtained was then summed 10-20 times. The data was stored in the bubble memory in the scope and transferred to a personal computer for further analysis.

In order to assess the character and strength of the cast-off vortex, data was gathered $25 \mathrm{~cm}$ upstream of the NACA $0012 \mathrm{~s}$ location after the test airfoil was removed 
from the wind tunnel. The suddenly pitched vortex generating airfoil produced a tightly rolled up vortex sheet with a viscous core of about one tenth the chord in diameter. Furthermore, the velocity profiles were also measured one chord to each side of the midspan plane and indicated that the vortex was two-dimensional within the instrumented portion of the test airfoil. Figure 5.6 represents the swirl velocity profile of the vortex.

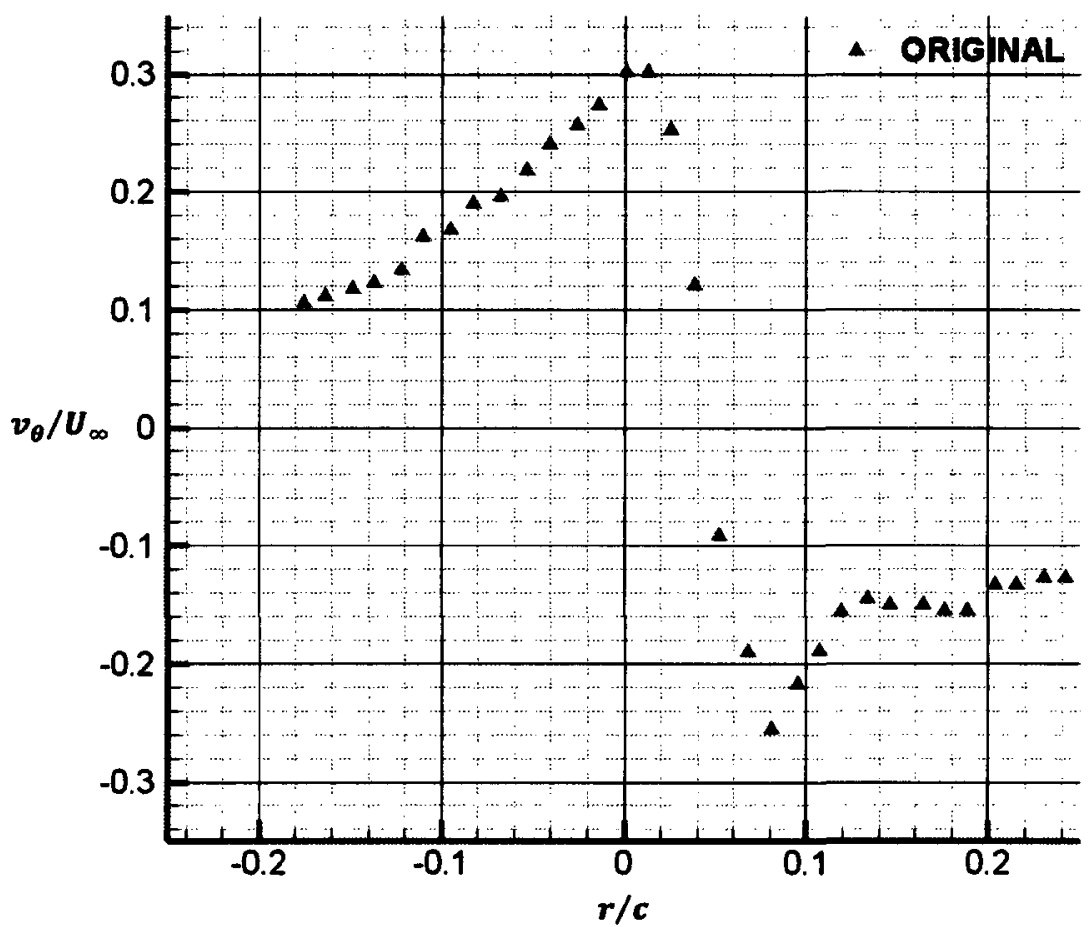

Figure 5.6: Swirl Velocity Profile of Vortex Generated by Straus et al. (1990)

In order to impose a vortex into ANSYS CFX 11.0, the data must be of the form which may be expressed by some mathematical relationship. This requires the data to be symmetric and centered about its own axis. The data in Figure 5.6 is neither centered nor symmetric. Furthermore, the data presented above is for a non-dimensional circulation of $\bar{\Gamma}=\Gamma / U_{\infty} c=-0.15 ;$ however, the lift and moment expressions used to validate the method were obtained for $\bar{\Gamma}=0.16$. In order to obtain a centered and symmetric profile 
with the correct $\bar{\Gamma}$ several steps must be taken. To begin with the data presented in the $(-x)(+y)$ plane of Figure 5.6 will be mirrored about the line $y=x$ while removing the original data in the $(+x)(-y)$ plane. This mirroring process was performed since the vortex will pass below the airfoil in the simulation, as opposed to above it as in the experiment. Therefore, the sense of the vortex must be the opposite in the simulation as in the experiment (which is accomplished through this mirroring process). The data must then be shifted so that the swirl velocity is zero at a radius of zero, this shift has the effect of decreasing the miss distance. The original and the shifted miss distances are presented in Table 5.4.

\begin{tabular}{|c|c|c|}
\cline { 2 - 3 } \multicolumn{1}{c|}{} & Original & Shifted \\
\hline$\left(y_{v} / c\right)[1]$ & -0.19 & -0.146 \\
\hline
\end{tabular}

Table 5.4: Miss Distances of the Vortex in Straus et al. (1990) (Original) and in the Simulation (Shifted)

The next alteration is to multiply the swirl velocity of the vortex in Straus et al. (1990) by a factor of $16 / 15$ to correct for the circulation (since there is a linear variation in circulation for pitch angle). Finally, the data must be mirrored about the line $y=-x$ to obtain the form of the velocity profile used in the LES analysis of the parallel blade vortex interaction. The final velocity profile is presented in Figure 5.7 along with the original data set to illustrate the changes which have been made. The altered data presented in Figure 5.7 may impose a vortex in the computational domain to obtain data related to the validation of the computational method.

Finally, Straus et al. (1990) presented data pertaining to the aerodynamic coefficients (lift and moment) throughout the interaction. This data shall validate the 
method and shall be presented in Section 5.9. Also presented in this section is the data which Straus et al. (1990) used to compare the experimental results which were obtained through implementing a discrete vortex method, these were provided by Renzoni (1987).

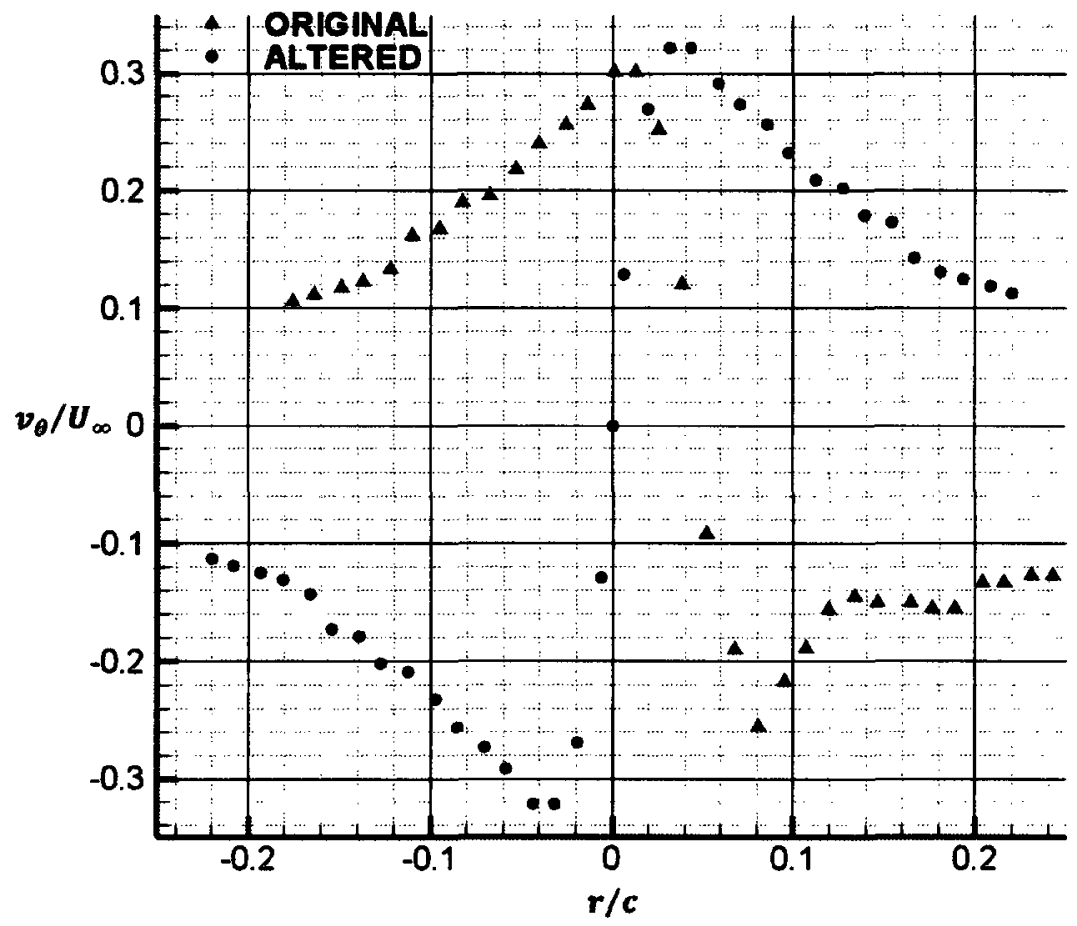

Figure 5.7: Original (red) and Altered (black) Straus et al. (1990) Vortex Velocity Profiles

The parameters which shall be used to initialize the domain may now be presented.

\subsubsection{Initialization of the Computational Domain}

A particular sequence must be followed prior to initializing the domain. The related sequence involves several steps. These steps (or simulations required for proper initialization) are as follows:

1) Uniform flow past the airfoil using RANS 
2) LES simulation for uniform flow past the airfoil initialized using the RANS solution results

3) Once the flow is fully developed, the uniform LES solution results are used to superimpose a vortex into the domain. This results in the requirement for parallel processing due to the computational requirements of this method. LES is then performed for the domain with the superimposed vortex.

The vortex imposed in the domain must be initialized based on the solution of the uniform flow using LES. This vortex is based on the altered data presented in Figure 5.7. Since the computational domains are all Cartesian, the $(u, v, w)$ components of velocity, as well as the static pressure, $p$ must all be initialized. As the Lamb-Oseen vortex model has been proven to provide good correlation with experimental data, it shall be used to superimpose the vortex into the computational domain. The swirl velocity profile for the Lamb-Oseen model which is outlined in Sub-Section 4.3.1 and is given as

$$
v_{\theta}(r)=\frac{\Gamma}{2 \pi r}\left[1-e^{\left(-\alpha\left(r / r_{c}\right)^{2}\right)}\right]
$$

Where $\alpha=1.25643$ and $r_{c}$ and $\Gamma$ are given in Table 5.3. Transforming the above expression from cylindrical coordinates to Cartesian coordinates yields

$$
\begin{gathered}
u(x, y)=U_{\infty}+\frac{\Gamma}{2 \pi r^{2}}\left(y-y_{v}\right)\left[1-e^{\left(-\alpha\left(r / r_{c}\right)^{2}\right)}\right] \\
v(x, y)=-\frac{\Gamma}{2 \pi r^{2}}\left(x-x_{v}\right)\left[1-e^{\left(-\alpha\left(r / r_{c}\right)^{2}\right)}\right] \\
w(x, y)=0
\end{gathered}
$$


where $y_{v}$ represents the miss distance of the vortex and is presented as the shifted data in Table 5.4. Also $x_{v}$ represents the initial location of the vortex with respect to the leading edge of the airfoil. The release location of the vortex for the following analysis is $x_{v}=-0.23 \mathrm{~m}$. The pressure started simply by the pressure field for the uniform flow past the airfoil obtained with LES and was allowed to evolve with time. In Equation ( 5.1 ) through Equation ( 5.3 ) the $r$ term is represented by

$$
r^{2}=\left(x-x_{v}\right)^{2}+\left(y-y_{v}\right)^{2}
$$

Figure 5.8 represents a comparison between the altered Straus et al. (1990) data with the Lamb-Oseen profile presented in Equation ( 5.1 ).

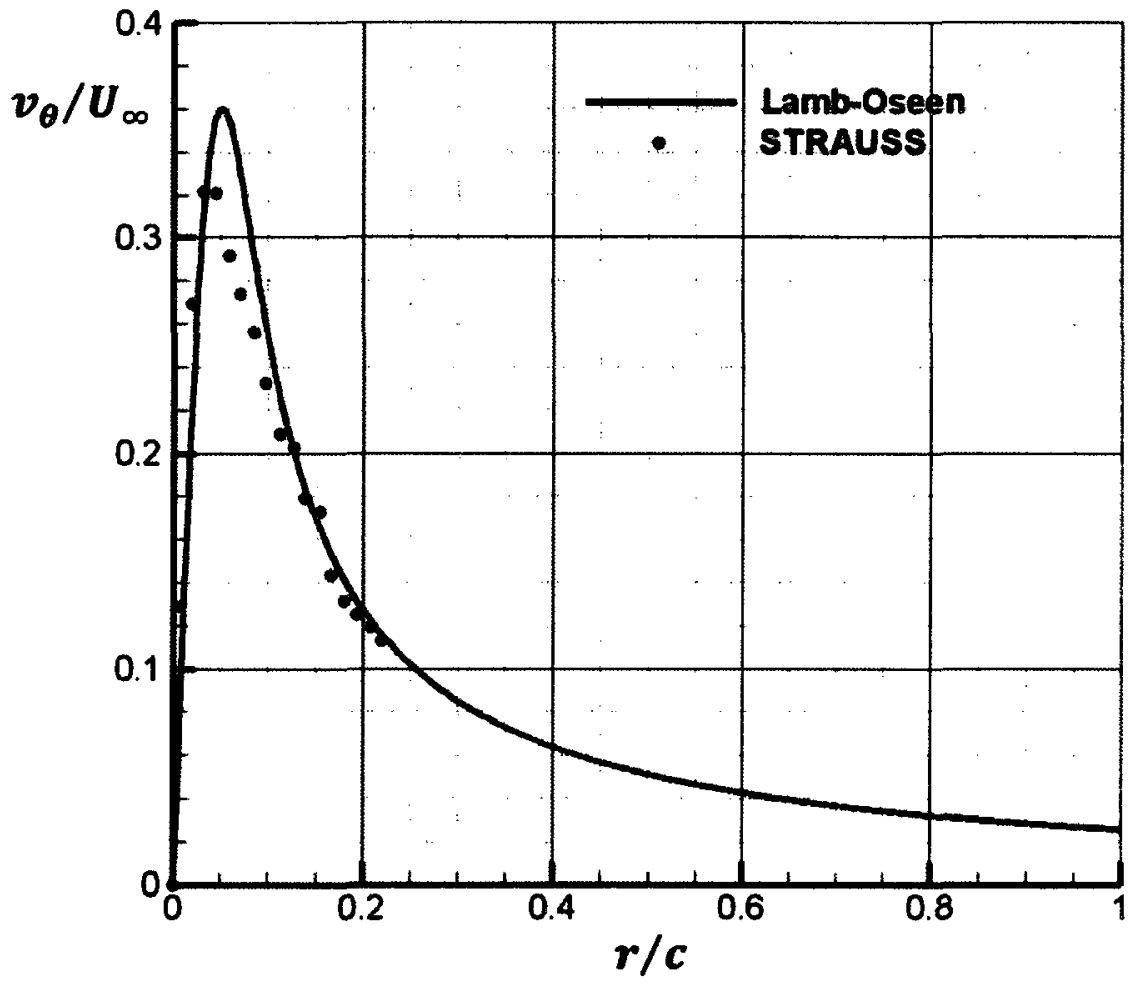

Figure 5.8: Comparison of the Swirl Velocity Obtained with the Lamb-Oseen Model and Straus et al.'s (1990) Altered Data 
In order to ensure the vortex does not influence the boundaries, a unit step function was employed so that the vortex had a diameter of only four chord lengths. If the vortex was large enough to reach the top/bottom of the domain (see Figure 5.9), the information would reflect from these walls towards the airfoil, thus causing unphysical results from the simulations. Finally, for the LES solutions, the velocity of the fluctuations was zero. In order to validate and verify the computational method, the boundary conditions imposed throughout the validation analysis shall now be outlined.

\subsubsection{Boundary Conditions Imposed on the Computational Domains}

Some nomenclature for the boundary conditions must be presented prior to presenting the boundary conditions for the computational domain. Figure 5.9 presents the computational domain with the associated boundaries indicated.

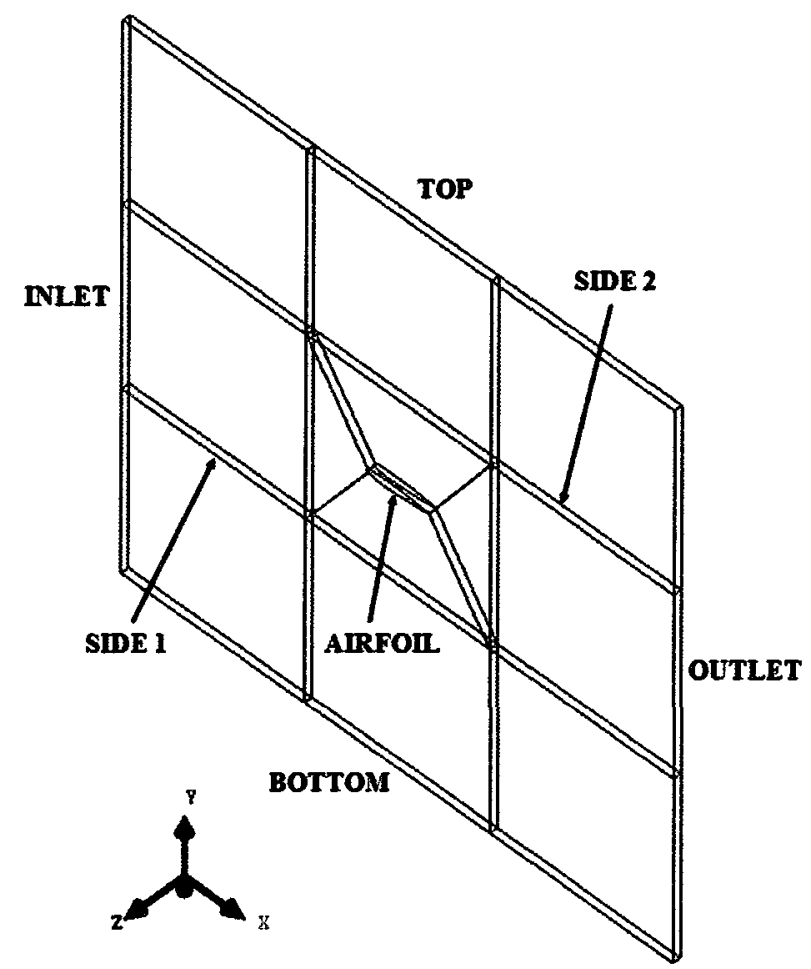

Figure 5.9: Boundaries for the Computational Domains 
Based on the definitions of the boundaries presented in Figure 5.9 as well as the data presented in Table 5.3, the boundary conditions may now be discussed. Table 5.5 provides the conditions imposed on the boundaries presented in Figure 5.9 as well as the associated value (if required).

\begin{tabular}{|c||c|c|}
\hline Boundary & Boundary Details & Value \\
\hline INLET & Speed Normal to Inlet & $12.2 \mathrm{~m} / \mathrm{s}$ \\
\hline OUTLET & Average Static Pressure & $1 \mathrm{~atm}$ \\
\hline AIRFOIL & Smooth Wall with No Slip & - \\
\hline TOP & Free Slip & - \\
\hline BOTTOM & Free Slip & - \\
\hline SIDE 1 & Symmetry & - \\
\hline SIDE 2 & Symmetry & - \\
\hline
\end{tabular}

Table 5.5: Boundary Conditions Used for the Validation Analysis

For the aforementioned verification study (i.e. using URANS), the inlet boundary also includes a $1 \%$ (of the freestream velocity) turbulent intensity associated with the boundary condition.

In order to validate the method, perform the domain/grid convergence, and to verify the method some basic aerodynamic properties were employed and shall now be presented.

\subsection{Definitions of the Aerodynamic Coefficients}

In order to obtain relevant information pertaining to the present analysis some basic aerodynamic parameters must be defined. Since the validation requires the use of the lift and moment coefficients, these shall be defined. Furthermore, the drag and 
pressure coefficients shall also be presented. The lift, drag and moment coefficients may be defined as

$$
\begin{gathered}
C_{l}=\frac{2 L^{\prime}}{\rho_{\infty} U_{\infty}^{2} c} \\
C_{d}=\frac{2 D^{\prime}}{\rho_{\infty} U_{\infty}^{2} c} \\
C_{m}=\frac{2 M^{\prime}}{\rho_{\infty} U_{\infty}^{2} c^{2}}
\end{gathered}
$$

Where $L^{\prime}, D^{\prime}$ and $M^{\prime}$ represent the lift per unit span (vertical force on the airfoil), drag per unit span (horizontal force on the airfoil) and the moment per unit span (torque about the airfoils leading edge (positive upwards)) respectively. Also, $\rho_{\infty}=1.185 \mathrm{~kg} / \mathrm{m}^{3}$ i.e. the density of air at $25^{\circ} \mathrm{C}$. Finally, the pressure coefficient is given by

$$
C_{p}=\frac{2\left(p-p_{\infty}\right)}{\rho_{\infty} U_{\infty}^{2}}
$$

where $p$ represents the static pressure at the point of interest and $p_{\infty}$ is the static pressure of the freestream, which is given as $1 \mathrm{~atm}$.

Based on the data presented in Section 5.3 through Section 5.5, the grid convergence may now be addressed.

\subsection{Grid Convergence Study}

To perform a grid convergence study, two structured grids of different sizes must be compared. This shall be performed by presenting the aerodynamic coefficients, $C_{l}$, $C_{d}$ and $C_{m}$ for these domains using the boundary conditions defined in Sub-Section 5.4.3. 
For this study, Domain A shall be compared to Domain B. Domain B is approximately double the size of Domain A which allows for a sufficient comparison to be made regarding the accuracy of the results. Domain A contains 760,464 nodes with the bulk of these clustered around the airfoil. The regions in which there is little effect from the vortex have been made coarser to minimize the computational resources spent on portions of the flow with little to no influence on the physics involved. Domain B has roughly double the number of nodes in the $x y$ plane, namely $1,365,648$ nodes. From Table 5.1 it can be seen that the nodes in the blocks which surround the airfoil have been significantly increased, thus providing greater resolution of the flow within this region. Furthermore, in the blocks downstream of the airfoil, the resolution has been increased in Domain B relative to Domain A. This was done to further capture the interaction between the vortex and the airfoils wake. A similar study was performed by llie (2008) using the same block structure with a more uniform structured grid throughout the entire computational domain in the sense that the resolution was consistent in each block surrounding the O-grid block containing the airfoil.

Figure 5.10 illustrates a comparison of the lift coefficient, $C_{l}$, as the vortex traverses from a non-dimensional upstream horizontal location of roughly half a chord from the leading edge (i.e. $(x / c)=-0.5)$ until the vortex is one chord downstream of the trailing edge of the airfoil (i.e. $(x / c)=2.0$ ). In a manner similar to Figure 5.10, Figure 5.11 presents a comparison between Domains A and B for the drag coefficient for the same range of vortex locations. Finally, the moment coefficient for the same vortex locations in Figure 5.10 and Figure 5.11 is presented in Figure 5.12. 


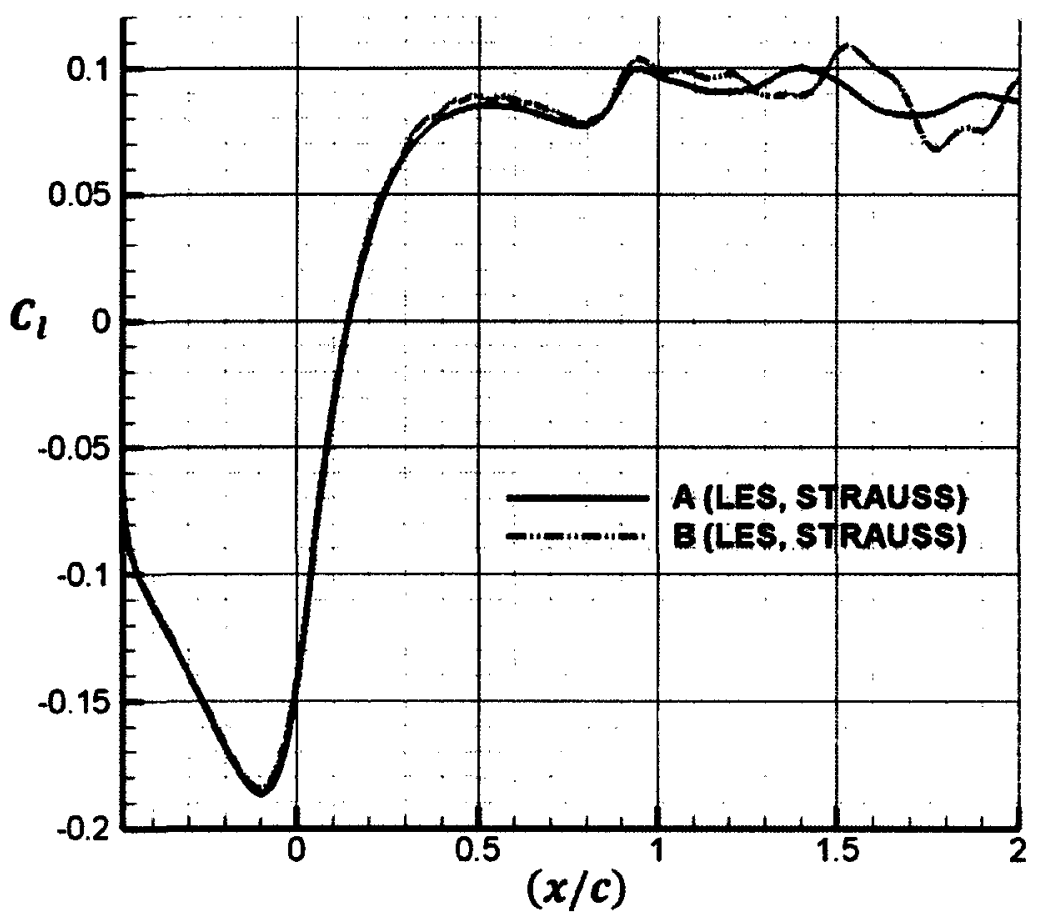

Figure 5.10: Lift Coefficient for Varying Vortex Location for Domains A and B

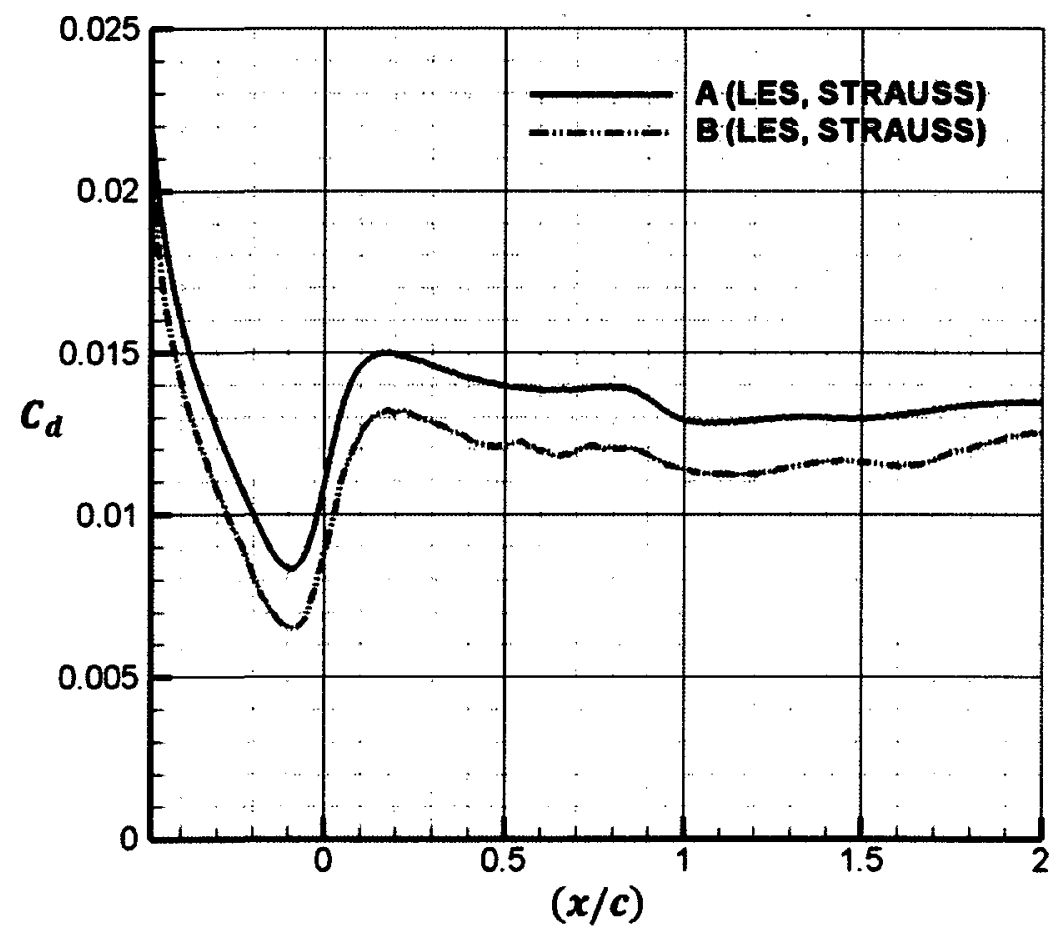

Figure 5.11: Drag Coefficient for Varying Vortex Location for Domains A and B 


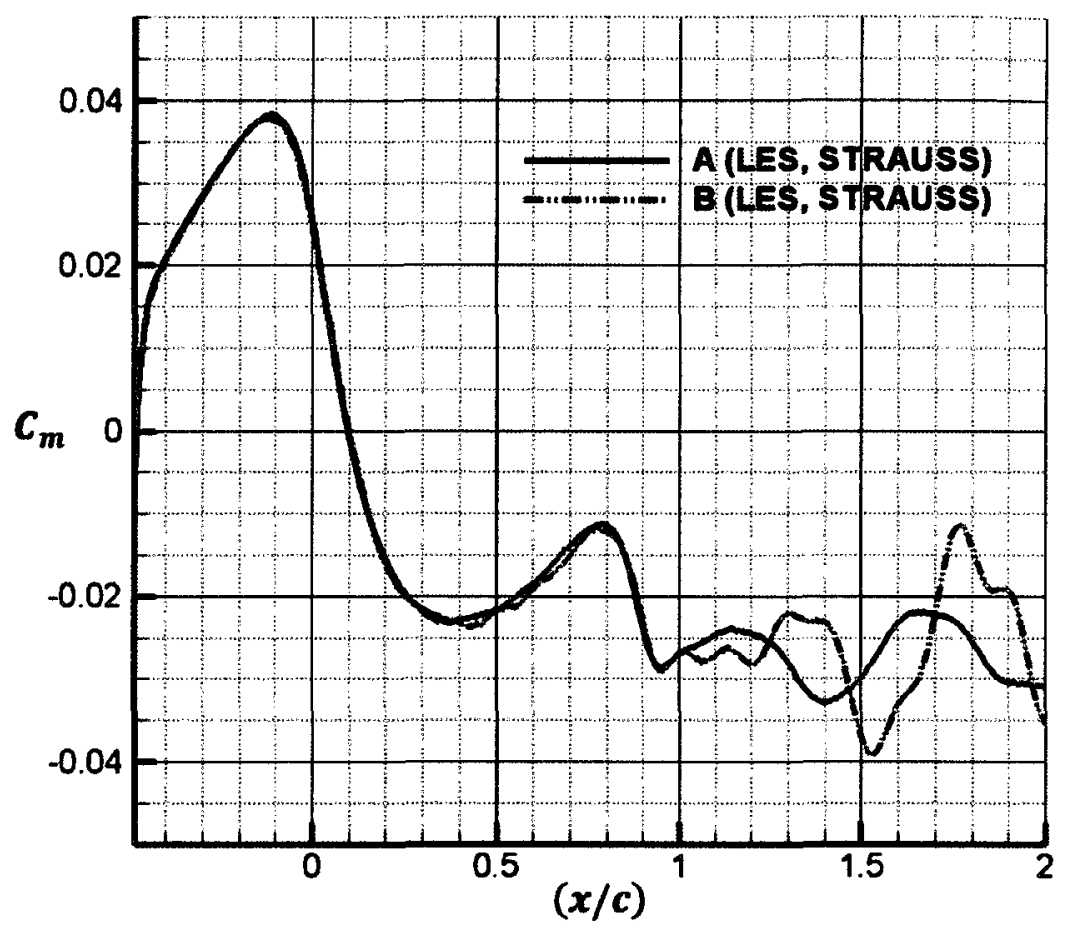

Figure 5.12: Moment Coefficient for Varying Vortex Location for Domains A and B

Upon examination of Figure 5.10 through Figure 5.12, several conclusions may be made regarding the number of grid points present in the domain as well as the structure of the grid in different regions. Figure 5.10 illustrates that regardless of the number of grid points (i.e. 760,464 (Domain A) or 1,365,648 (Domain B), grid convergence is obtained for the lifting force during the blade-vortex interaction defined by Straus et al. (1990). This is a result of the higher resolution of the grid within the region of the airfoil, which allows the vortex to be more accurately defined between each node (i.e. smaller distances between which the vortex velocity profile is linearly interpolated). This is also true for the spike at $(x / c)=0.5$. The convergence remains until the vortex core approaches the trailing edge of the airfoil. At this point a divergence between Domain A and Domain B may be noticed in the lift. Since the Kármán vortices 
shed off the trailing edge seem to differ from one domain to the next, the structure of Domain B within the wake region of the domain may have some influence on this deviation. This is expected as Kármán vortices shed periodically after some event initiates them. As a result, differences between the two domains downstream of the airfoil trailing edge may be expected. Similar arguments may be made for the moment coefficient (see Figure 5.12). Additionally, it may be concluded that the fluctuations in the moment coefficient are dictated by the pressure distribution which is responsible for the generation of the lift force (i.e. these coefficients are inherently linked) as opposed to the drag force during the airfoil-vortex interaction.

Figure 5.11 illustrates the drag coefficient as the vortex convects from its initial upstream location to its final downstream location. Notice that the curve for Domain B always predicts less drag than Domain A. As a result, there is no convergence of the drag forces during the interaction. But, since the investigation selected to validate the use of LES for AVI studies only provides data for the lift and moment coefficients, it is acceptable for this parameter to have no convergence as there is no data to validate the drag generated during AVI. As Domain B has far superior resolution in the block surrounding the airfoil (i.e. the O-grid block), the difference between the drag coefficients for Domain A and B may be attributed to the better resolution of the skin friction component of drag due to the superior resolution of the boundary layer. This causes a decrease of the skin friction component of drag.

Based on the above discussion and the arguments made throughout, despite the deficiencies in predicting the variation of drag, Domain A shall be employed for the 
following analysis. The main reason for this is that Domain A provides accurate results until the vortex reaches the trailing edge of the airfoil. In blade-vortex interaction analyses, the region of interest with respect to the unsteady aerodynamic forces is the region in which the vortex is just upstream of the airfoil (i.e. at the release location for the simulations performed) until it leaves the trailing edge of the airfoil. As it provides the magnitude of the increase in lift (i.e. the spike which may be seen in Figure 5.10) this region is of interest. This region is also responsible for the highly focused noise as well as the intense noise generated during a blade-vortex interaction. Furthermore, the simulations performed each used 95,000 time steps. Those performed for Domain A were completed in roughly 9 days; however, those completed using Domain B took approximately 21 days using the cluster of 20 parallel computers. Therefore, because of the difference in computational costs as well as the sufficient resolution of the results in the region, Domain A shall be used to perform the domain convergence study.

\subsection{Domain Convergence Study}

Based on the results from the grid convergence analysis the thickness of the converged grid (i.e. the thickness of Domain A, $t=0.12 c$ ) shall be doubled to obtain Domain $C(t=0.24 c)$. The number of nodes in the $z$ direction shall also be doubled. This process is outlined in Table 5.1 upon examining the $\Delta z$ values for both Domain A and $\mathrm{C}$. The number of nodes in the $x y$ plane for Domain $\mathrm{C}$ remains the same as Domain A. This analysis thus simply provides information as to whether the thickness of the domain plays a role in the accuracy of the aerodynamic coefficients, $C_{l}, C_{d}$ and $C_{m}$ 
produced for the same boundary conditions as presented in Sub-Section 5.4.3. It is worth noting that Domain C contains $1,520,928$ nodes.

Using the same guidelines as Figure 5.10 through Figure 5.12, the lift, drag, and moment coefficients shall be presented as the vortex location is varied in Figure 5.13 through Figure 5.15.

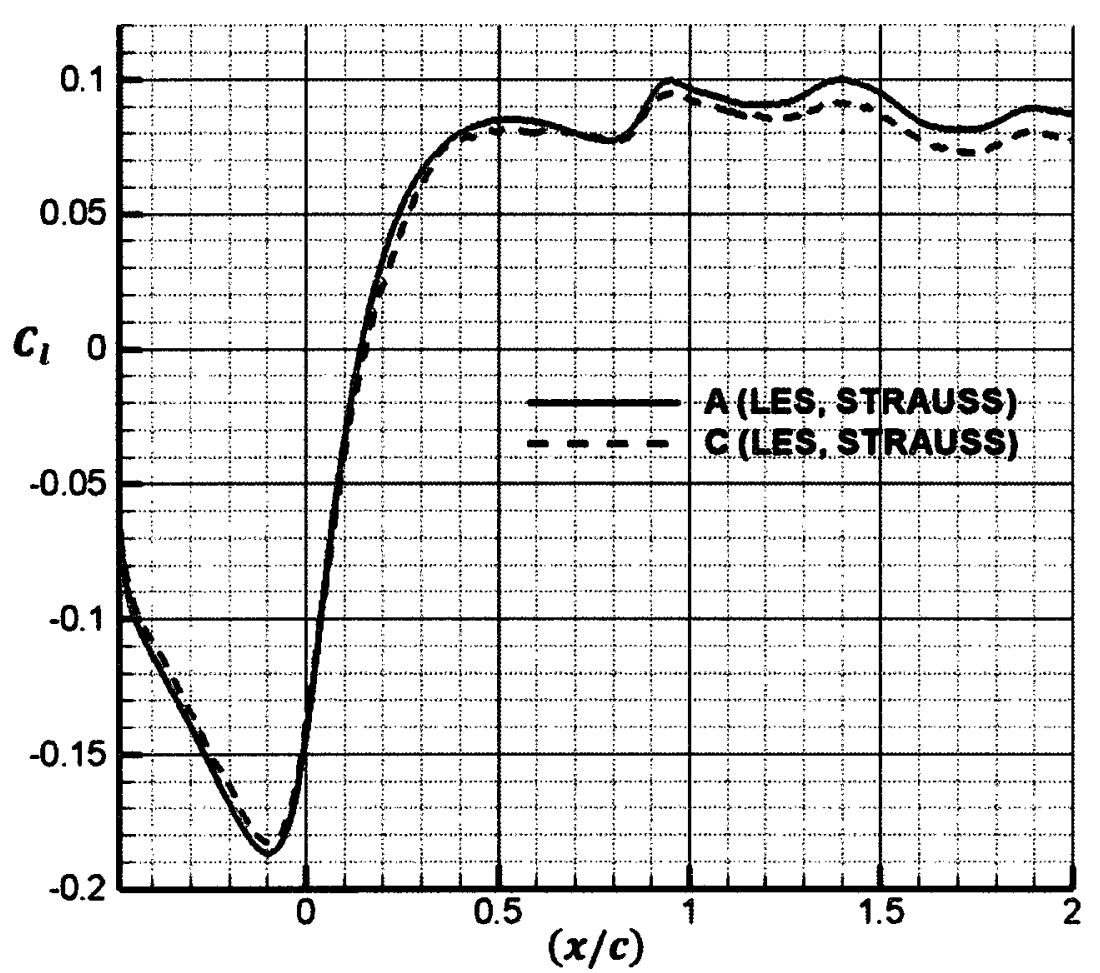

Figure 5.13: Lift Coefficient for Varying Vortex Location for Domains $A$ and $C$ 


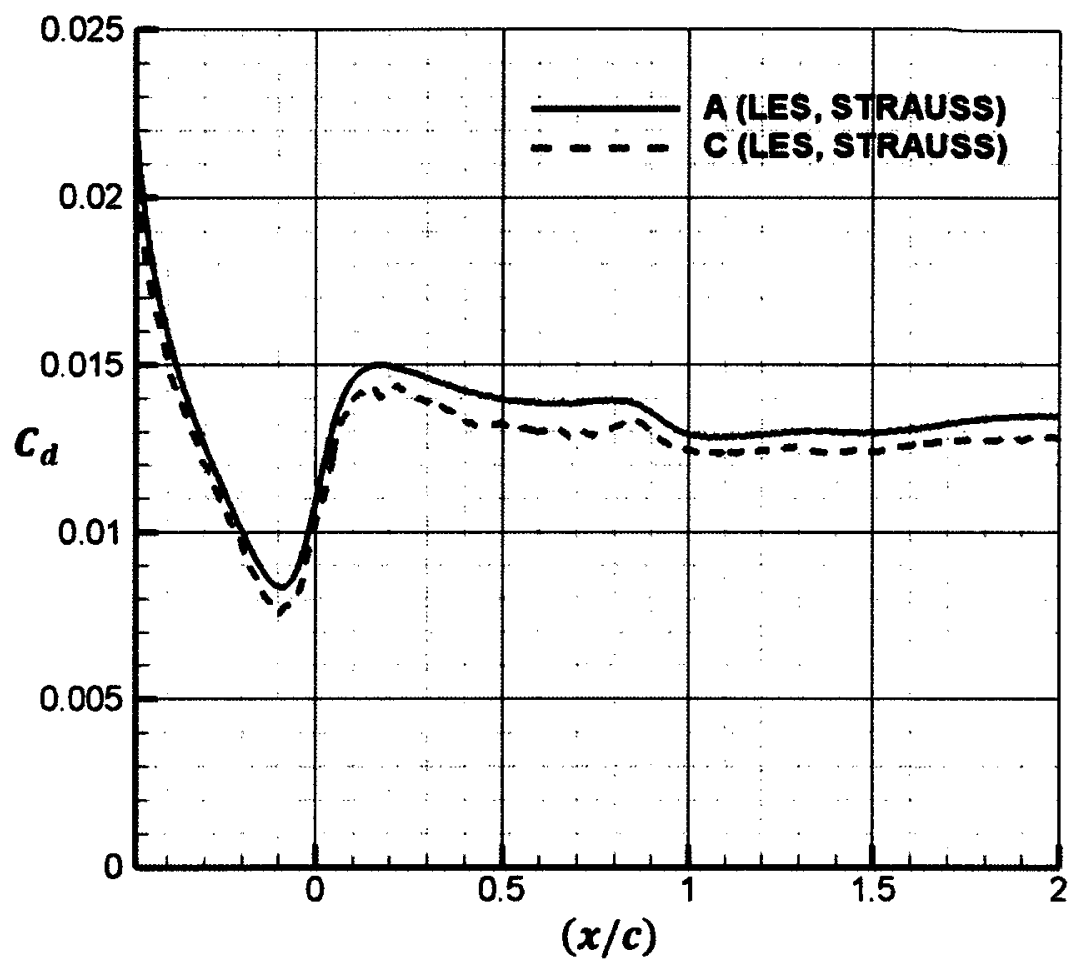

Figure 5.14: Drag Coefficient for Varying Vortex Location for Domains $A$ and $C$

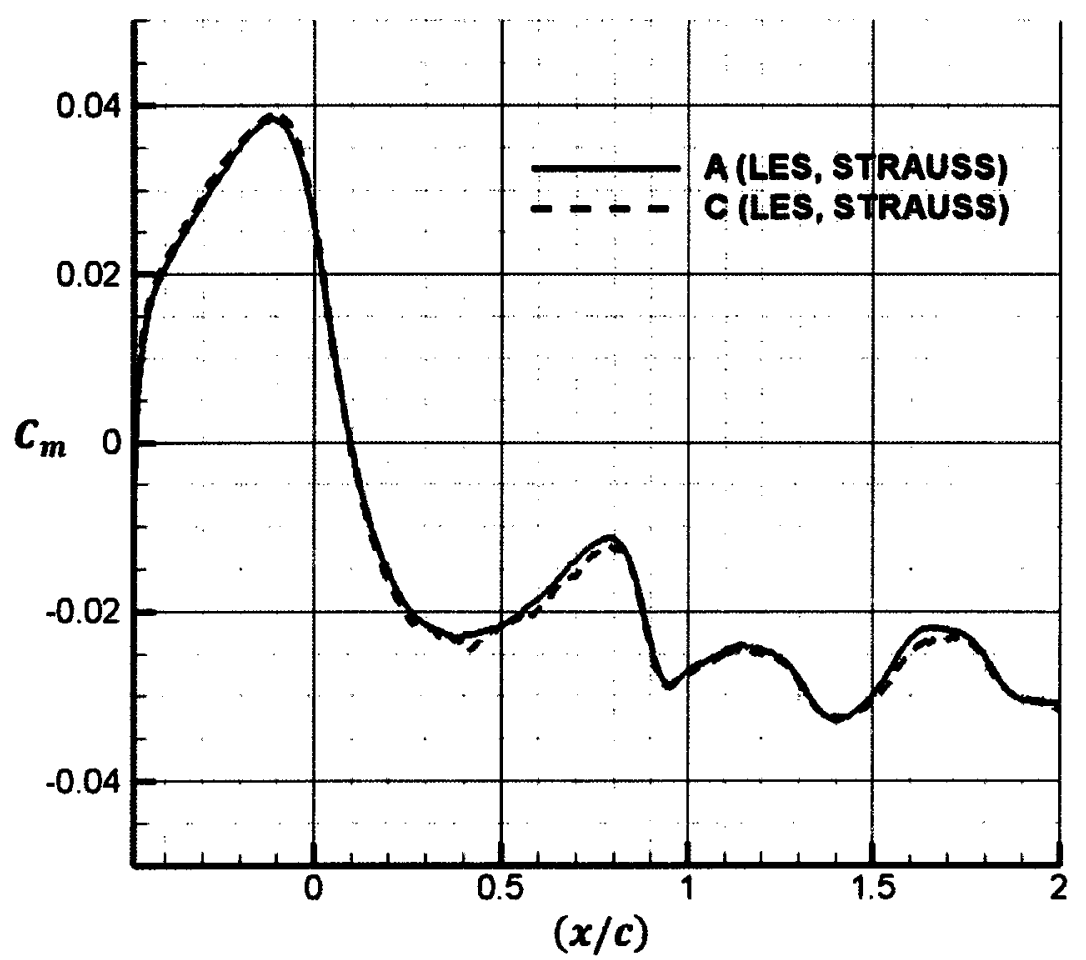

Figure 5.15: Moment Coefficient for Varying Vortex Location for Domains $A$ and $C$ 
Based on Figure 5.13 through Figure 5.15, several conclusions relating to the thickness of the domain used to complete the validation and verification study may be made. Figure 5.13 shows that, until the vortex approaches the trailing edge of the airfoil, the results are quite similar. Some differences in lift predicted by Domain A and Domain $\mathrm{C}$ are evident in Figure 5.13 from the release location of the vortex until it approaches the trailing edge. As the vortex core approaches the airfoils leading edge, there is a slight deviation in the trough value of the lift between Domains A and C. This may be attributed to numerical error which may also be seen when the vortex is at the half chord location. It appears that there is a slight three dimensional effect during the airfoil-vortex interaction particularly when the vortex approaches the trailing edge of the airfoil. This is demonstrated by a slightly lower value of the lift coefficient for Domain $\mathrm{C}$ as the vortex traverses the wake region. Therefore, the wake which is a three-dimensional phenomenon, has more room in Domain $\mathrm{C}$ to expand. As the vortex core interacts with the wake, the interaction is slightly decreased in the wider domain. However, the difference is quite small (i.e. $1 \%$ difference in the lift coefficient when the vortex is one chord downstream of the airfoils trailing edge). As a result, the results obtained by the thinner domain regarding lift (i.e. Domain A) are quite reasonable. As per the grid convergence study, similar arguments between the lift and moment coefficients may be used, even though the expansion of the domain in the third dimension seem to have less of an effect on the moment coefficient than the lift coefficient, as seen in Figure 5.15.

Figure 5.14 illustrates the drag coefficient as the vortex convects from its initial upstream location to its final downstream location. As per the grid convergence study, the 
wider domain (i.e. Domain C) predicts lower drag forces throughout the entire airfoilvortex interaction than the thinner domain (i.e. Domain A). The decrease in drag in the thicker domain may be attributed to the increased number of nodes (and hence resolution of the results) in the z-direction (disregarding thickness). Perhaps increasing the number of nodes in this direction would lead to a better resolution of the boundary layer which causes a lower component of the skin friction drag, thereby decreasing the total drag variation induced during the interaction.

Based on the above discussion and the arguments made throughout, Domain A shall be employed for the verification and validation analysis, despite the insufficiencies in predicting the variation of drag. Noting that similar effects were seen for the wider domain (i.e. Domain C) to the domain with a larger resolution of the grid (i.e. Domain B), similar conclusions related to the physics may be made for the domain convergence study. Furthermore, the simulations performed each used 95,000 time steps. Those performed for Domain A were completed in roughly 9 days; however, those completed using Domain $\mathrm{C}$ took about 25 days using the cluster of 20 parallel computers. Therefore, due to the difference in computational costs as well as the sufficient resolution of the results in the aforementioned region, Domain A shall be used to perform the verification and validation studies.

\subsection{Verification of the Computational Method}

The domain deemed appropriate from both a physical/numerical accuracy and computational load point of view by employing the results of the grid and domain convergence analysis will be used to compare the LES results of an AVI event with the 
results of an AVI event obtained by an SST based URANS simulation. This was performed to see how the predictions of the aerodynamic coefficients from the URANS method, which is a method commonly used for AVI investigations, compare to the aerodynamic coefficients obtained with the LES method, which has only recently been used for AVI analyses. Domain A was used to compare the results from the LES simulations with the SST based URANS simulations. The SST based URANS simulations were performed using the same time step and Domain A; the CFL criterion for an LES simulation was enforced. The boundary conditions for each simulation are presented in Sub-Section 5.4.3.

Based on the axes definition established for Figure 5.10 through Figure 5.12 (refer to Section 5.6), similar results may be obtained for the comparison of the system of equations employed for the mitigation of the blade-vortex interaction effect study. Figure 5.16 through Figure 5.18 represent plots of the lift, drag and moment coefficients respectively (i.e. $C_{l}, C_{d}$ and $C_{m}$ ) as the vortex convects downstream from its initial location to the one chord downstream of the trailing edge of the airfoil. 


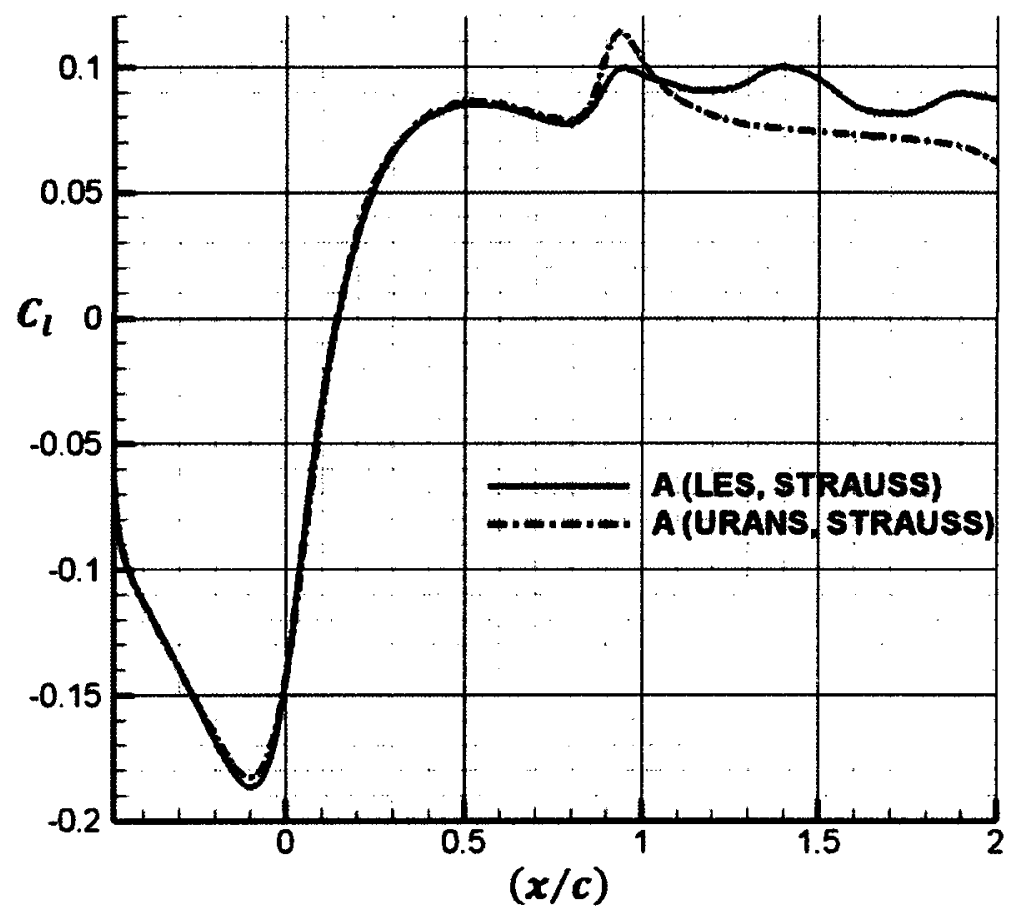

Figure 5.16: Lift Coefficient for Varying Vortex Location for Domain A Using LES and the SST Based URANS

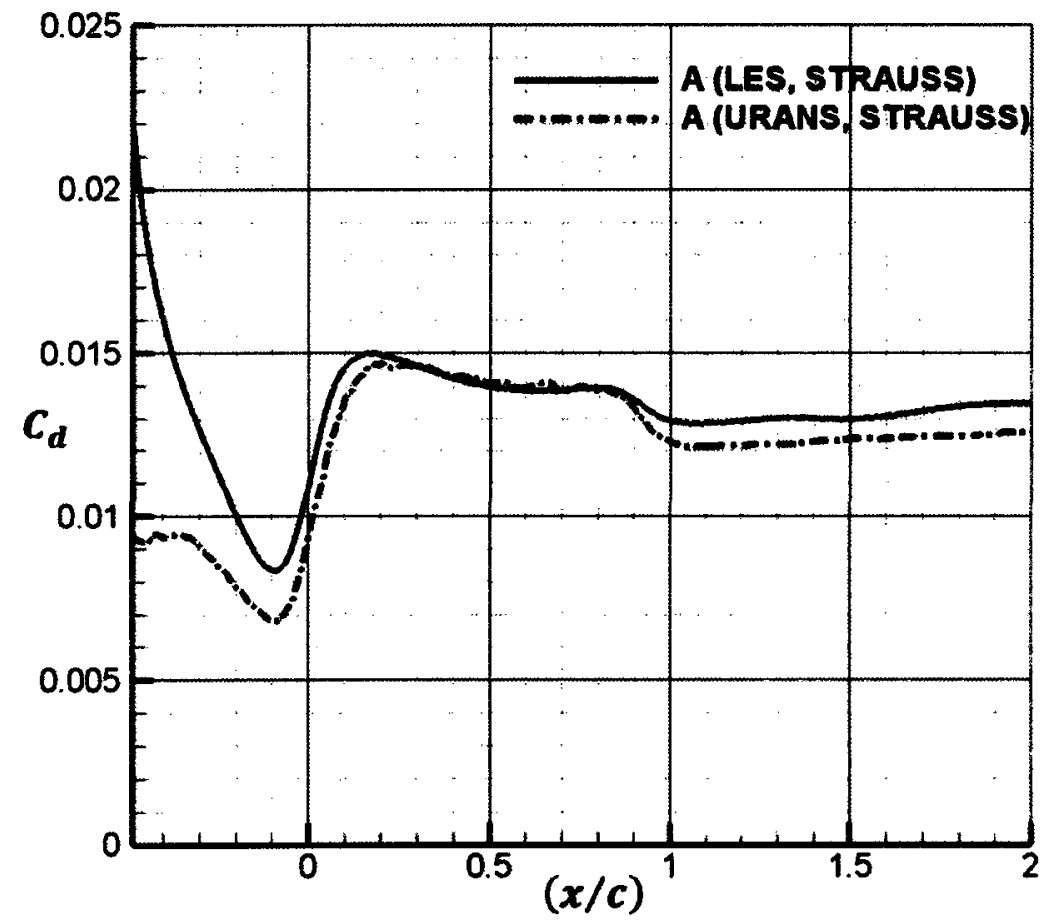

Figure 5.17: Drag Coefficient for Varying Vortex Location for Domain A Using LES and the SST Based URANS 


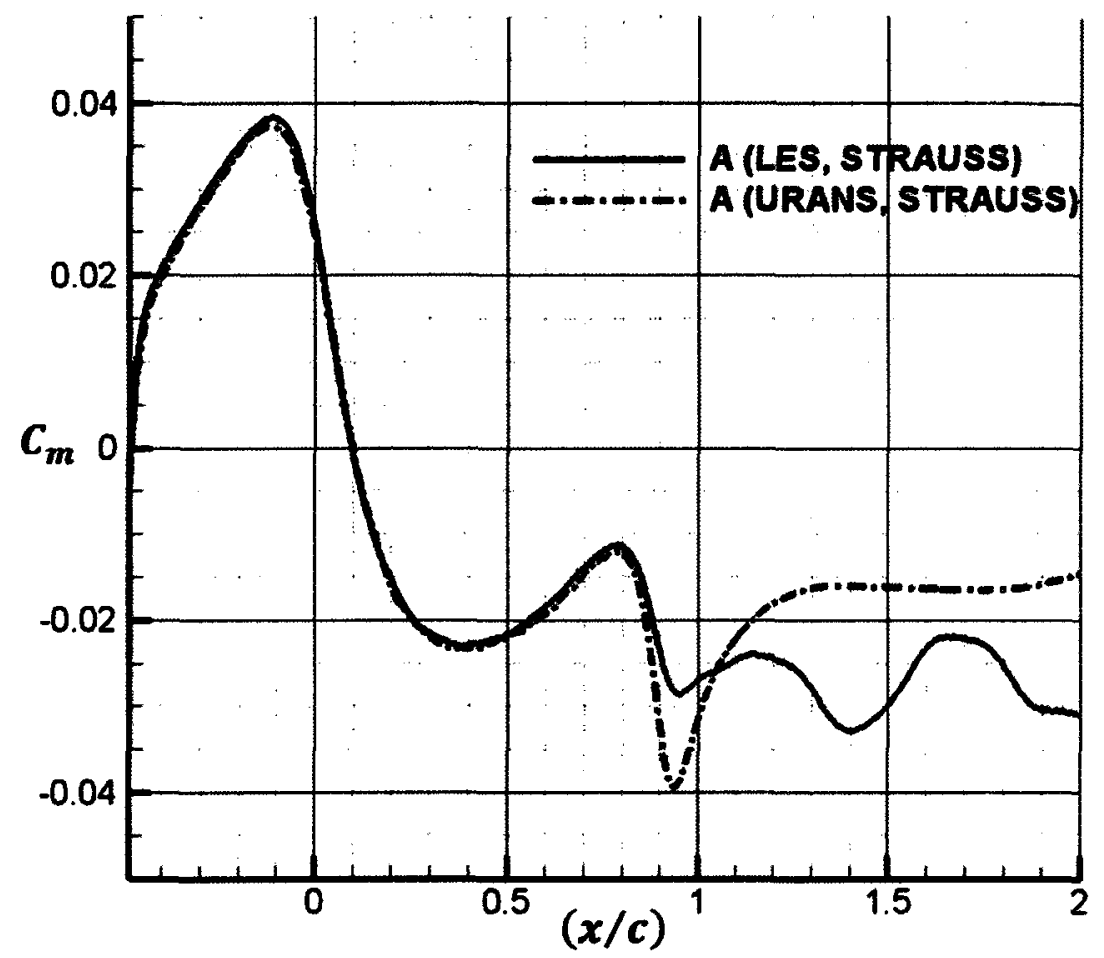

Figure 5.18: Moment Coefficient for Varying Vortex Location for Domain A Using LES and the SST Based URANS

Furthermore, a notable difference may be seen in the interaction between the vortex and the wake of the airfoil as the vortex traverses to its final location downstream of the trailing edge. As a result Figure 5.19 illustrates the velocity fields for both the LES results and the SST based URANS results at several locations of the vortex. These positions are presented in half chord progressions with respect to the vortex location from its starting point; i.e. $1 / 2$ chord upstream of the airfoils leading edge, to the location where the vortex is one chord downstream of the airfoils trailing edge. 
Velocity [m $\left.s^{\wedge}-1\right]$
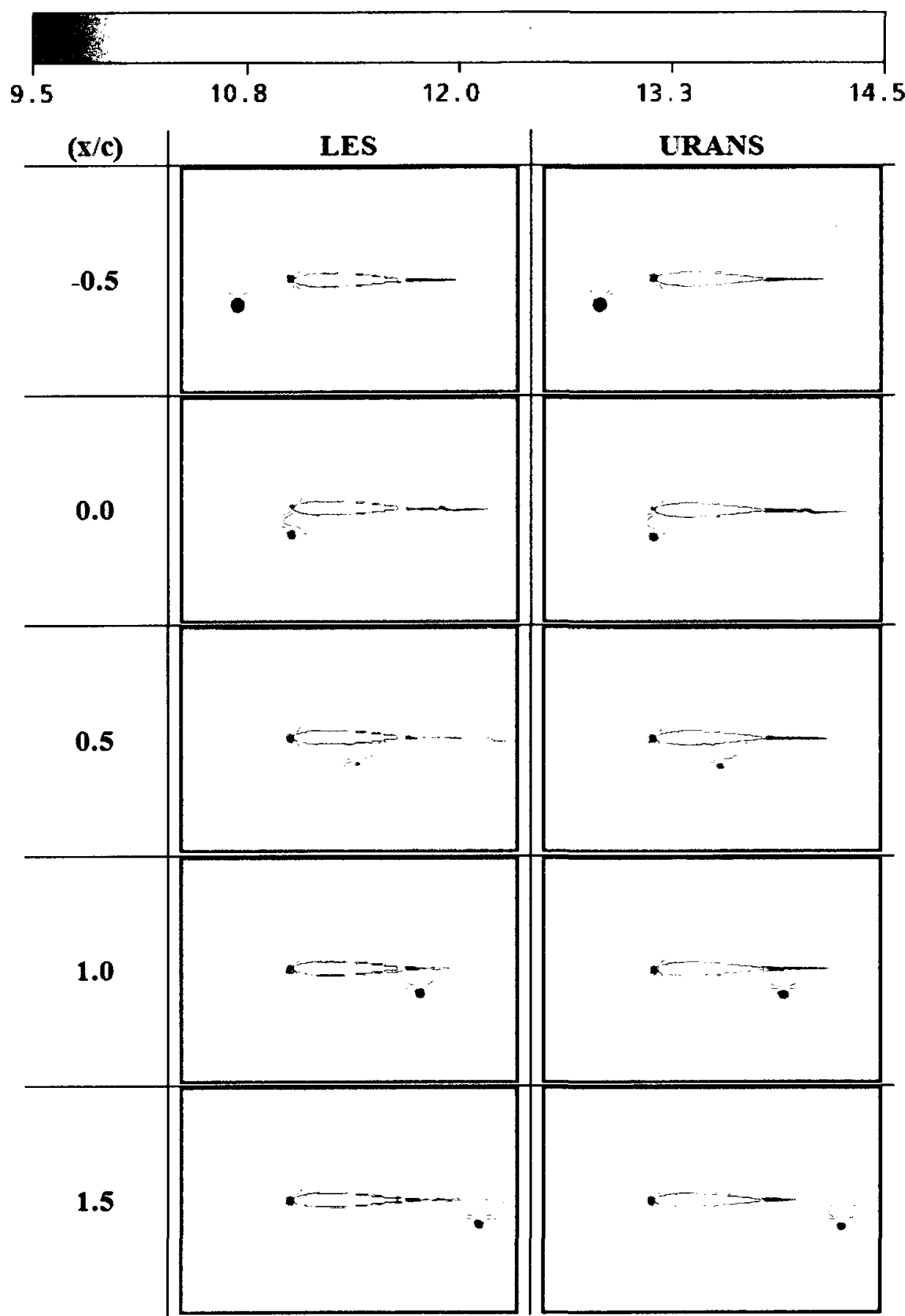

Figure 5.19: Velocity Field Obtained from a) an LES and b) a URANS Simulation of the BladeVortex Interaction 
Figure 5.16 through Figure 5.18 present the aerodynamic coefficients obtained during the airfoil-vortex interaction using Domain A with LES and an SST based URANS. As Domain A was deemed the convergent domain and grid based on the discussions presented in Section 5.6 and Section 5.7 it was be used for this verification study. It is noteworthy that the LES simulation modeled only the small scales of turbulence using the Smagorinsky model with the Van Driest wall damping function based on the theory presented in Chapter 3:. Whereas the URANS simulation modeled the entire turbulence field by the SST model, which is a weighted combination of the $k-\varepsilon$ and $k-\omega$ models. This difference implies that LES shall capture the structures of the wake and the boundary layer with superior accuracy as compared to the URANS approach. Moreover, the CFL criterion (outlined in Section 5.3) for the URANS based simulation, which is a requirement of an LES based simulation, was also employed (i.e. $\mathrm{CFL}<1)$ using the same time step $\left(1 \times 10^{-6}\right.$ seconds) and structured grid (refer to Figure 5.4) as per the LES simulation.

Several conclusions may be reached by comparing the lift coefficient obtained using both LES and URANS, refer to Figure 5.16. Firstly, it becomes evident that when the vortex convects downstream from its release location until it approaches the trailing edge (as per all other investigations), there is good agreement between the LES and URANS data. This implies that during the strong interaction between the airfoil and the vortex there exists very little influence from the turbulent nature of the flow. This in turn implies that the boundary layer and wake during this portion of the interaction do not seem to influence the lift coefficient in any way. But, as the vortex passes the trailing 
edge (i.e. as it traverses into the highly turbulent portion of the domain), there are noticeable differences. LES is able to capture the wake structure with far superior resolution to URANS, the Kármán vortices discussed in Section 5.6 may be realized for the LES results (see Figure 5.19a). However, since the URANS method does not capture the turbulent fluctuations in the wake due to the averaging process, as the vortex convects downstream of the airfoils trailing edge, it converges back to its zero lift condition (i.e. for a uniform flow with a symmetric airfoil at zero angle of attack) much faster than the LES results. Also, when the vortex is at the trailing edge of the airfoil, the URANS results predict a secondary rise in lift absent in the LES results. This may be attributed to the weaker Kármán vortices formed in the URANS simulation. Thus, as the vortex leaves the airfoil, there is a substantial interaction in the URANS simulation resulting from the interaction with the wake. Such interactions imply that the boundary layer and wake resolution plays a role in the interaction as can be seen in Figure 5.19b. Similar conclusions may be made for the moment coefficient for the URANS results as can be seen in Figure 5.18.

Figure 5.17 illustrates the drag coefficient as the vortex convects downstream for LES and URANS solutions using Domain A and the conditions defined by Straus et al. (1990) study. Notice that the drag coefficient for the URANS simulation, as the vortex is initially released (i.e. $(x / c)=-0.5)$ is grossly under-predicted compared to the LES simulation. This remains true until the vortex arrives at $(x / c)=0.25$ where the URANS solution determines the drag in a manner identical to the LES simulation. As the vortex approaches the airfoil (i.e. for $(x / c) \in[-0.5,0.25])$ the use of URANS is unjustified on 
account of the gross under-prediction of the drag. This may be attributed to the mean flow averaged values interacting with regions of high pressure gradients, which implies that as the vortex is upstream of the airfoil employing URANS provides unreasonable results for the drag forces on the airfoil. Further discrepancies may be caused by the presence of freestream turbulence in the URANS simulations and the absence of freestream turbulence in the LES simulations. As the vortex reaches the trailing edge (i.e. $(x / c)=0.8)$ the URANS simulation begins to underestimate the drag forces on the airfoil during the interaction. These results indicate that the turbulence models employed have no impact on the drag estimation, while the vortex is directly below the airfoil (at least for $(\mathrm{x} / \mathrm{c}) \in[0.25,0.8])$. However, as the vortex traverses the airfoil's wake (i.e. for $(x / c) \geq 0.8)$ the turbulence models affect the drag estimation. Employing the SST model for the turbulence in the URANS simulation appears to change the drag estimate during the wake interaction; this may be attributed to the use of both the $k-\varepsilon$ and $k-\omega$ models. The $k-\omega$ model provides superior turbulence prediction in the region near the walls, whereas the $k-\varepsilon$ model provides a better model of the turbulence in the freestream. As a result, the use of the $k-\omega$ model in the SST approach in the wake region may contribute to the under-prediction of drag.

Despite the similarities between the results of a URANS simulation and LES under the same conditions, LES is the preferable method for an airfoil-vortex interaction. As previously discussed, in the URANS simulation the Courant number was less than unity (as is the requirement for LES), which most likely caused the fairly accurate results (based on the above discussions). Due to the time steps used, there was no reduction in 
the computational load through the URANS approach when compared to LES (i.e. they each took 9 days on the $20 \mathrm{CPU}$ cluster to complete). Since there is no difference in computational time LES is the advisable simulation as it provides better resolution of the flow physics throughout the AVI event (this may be seen in the velocity fields presented in Figure 5.19). Furthermore, the URANS approach was not capable of capturing the Kármán vortices that interact with the blade tip vortex and caused fluctuations in both the lift and moment coefficients, while LES captured these flow structures. As a result, LES shall be employed on Domain A to be validated against the experimental results obtained by Straus et al. (1990), which shall now be discussed. Also, the LES results shall be compared to the discrete vortex method results using the same inflow conditions which were obtained by Renzoni (1987).

\subsection{Validation of the use of LES for Blade-Vortex Interaction Simulations}

Now that a convergent grid and domain has been selected (i.e. Domain A) as well as the use of LES, the converged solution and the computational method must now be validated. This will be done by comparing the lift and moment coefficients, $C_{l}$ and $C_{m}$, with the conditions defined by Straus et al. (1990) (refer to Section 5.4). The data obtained experimentally by Straus et al. (1990) is given in Figure 5.20. 


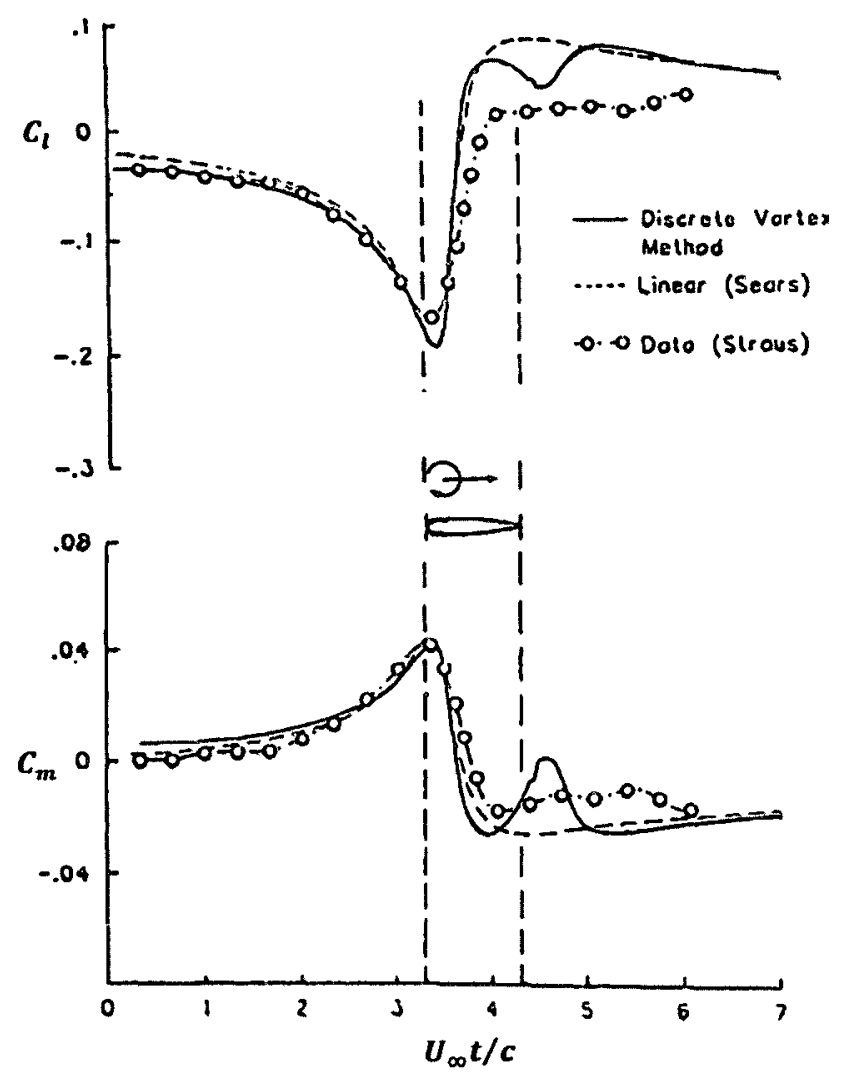

Figure 5.20: Experimental Data (Circles) Obtained by Straus et al. (1990) and Discrete Vortex Method Data (Solid Line) Obtained by Renzoni (1987)

Converting the $\mathrm{x}$-axis from non-dimensional time, $\hat{t}=U_{\infty} t / c$, to the position of the vortex with respect to the airfoil's leading edge, i.e. $(x / c)$, for each the Renzoni (1987) and Straus et al. (1990) data allows a comparison to be made with the data obtained through simulation. The data was first extracted from the figures from Straus et al. (1990) using a plot digitizer. The x-axis data was then converted in Microsoft Excel. The data presented at $\hat{t}=0$ was used as the distance between the two airfoils, presented in Figure 5.5. Based on the above discussion, the results for $C_{l}$ and $C_{m}$ using the same xaxis definition as Figure 5.10 and Figure 5.12 are provided in Figure 5.21 and Figure 5.22 . 


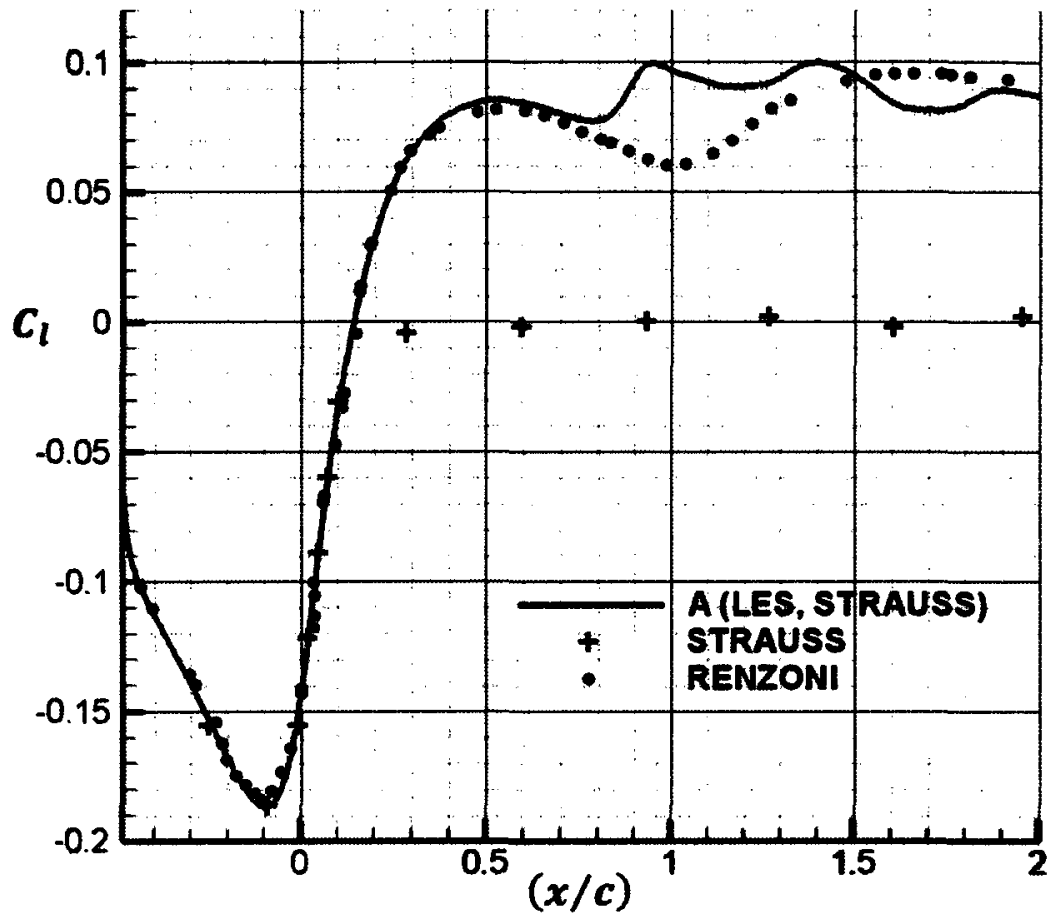

Figure 5.21: Lift Coefficient for Domain A Simulation (red line), Straus et al.'s (1990) Experimental Data (blue +) and Renzoni's (1987) Inviscid Discrete Vortex Method (black o)

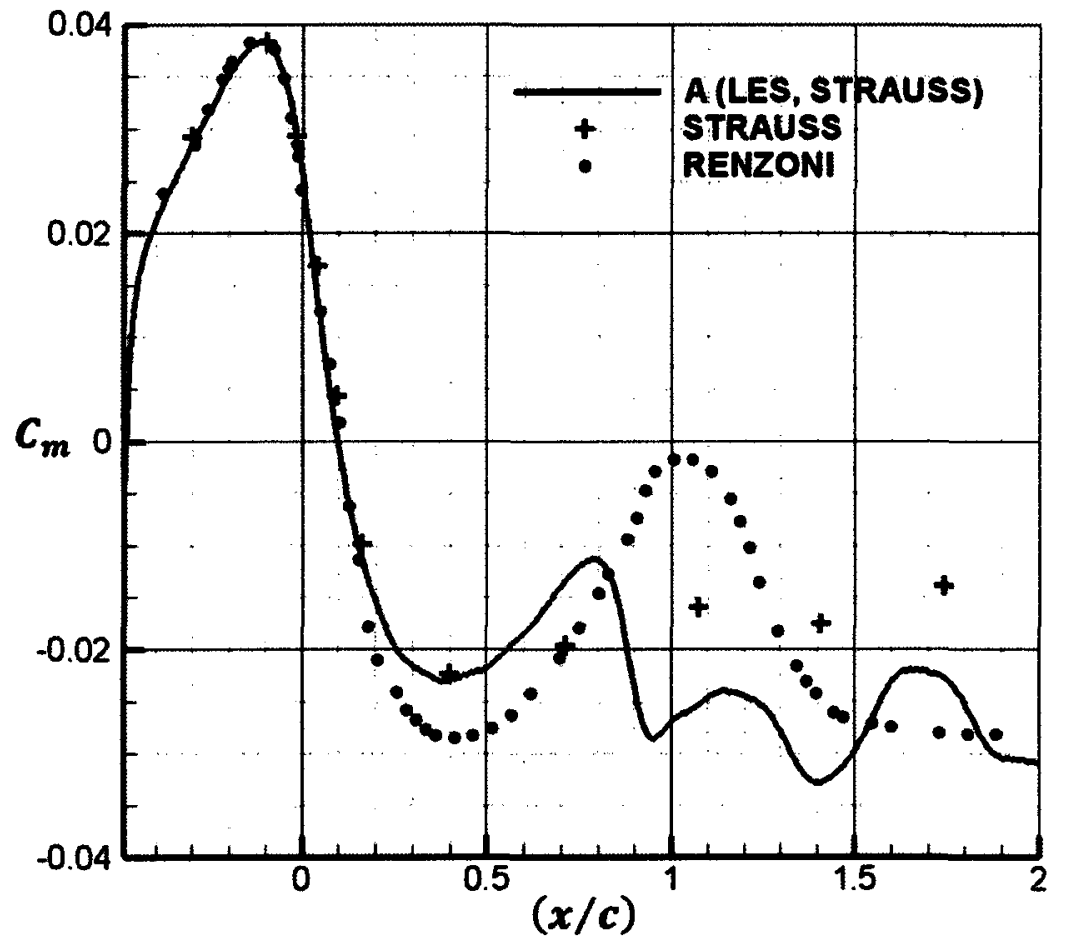

Figure 5.22: Moment Coefficient for Domain A Simulation (red line), Straus et al.'s (1990) Experimental Data (blue +) and Renzoni's (1987) Inviscid Discrete Vortex Method (black o) 
Figure 5.21 and Figure 5.22 present the lift and moment coefficients obtained during the airfoil-vortex interaction of Domain A using LES along with the experimental results presented by Straus et al. (1990) and the results obtained by Renzoni (1987) with a discrete vortex method. The results presented in Figure 5.21 shall now be discussed beginning with the differences between the LES simulation and experimental results. Notice that the lift coefficient obtained through simulation and experiment agree from the simulations release location until just after the vortex passes the leading edge of the airfoil (i.e. $(x / c) \in[-0.5,0.1])$. After this point the experimental results bifurcate from the results obtained by the LES simulation, most notably during most of the interaction (i.e. $(x / c) \in[0.1,2])$ the variation in lift predicted experimentally is zero. A zero lift coefficient is obtained for a symmetric airfoil at zero angle of attack in a uniform flow field. As a result, a lift coefficient of zero is expected after the vortex no longer influences the velocity/pressure distributions over the airfoil, i.e. when it sufficiently downstream of the airfoils TE. This occurs in the LES simulation results when the vortex is two chords downstream of the airfoil, even though it occurs immediately after the vortex passes the leading edge in the experiment as can be seen in Figure 5.21. Several reasons for the differences in the lift coefficient shall now be conjectured.

Unlike the simulation vortex, the experimental vortex may be conjectured to be dispersed by the blade in the third dimension (i.e. energy transferred into the z-direction). Since the boundary conditions on the side walls are those of symmetry (which do not allow energy transfer in the z-direction), this may result in a portion of the differences 
seen in Figure 5.21 between the simulation and the experiment. The experimental results for lift appear to represent a system critically damped due to the diffusion of the vortex, which because of the boundary condition limitations cannot be captured by the simulation. This diffusion may be modeled in the simulation by employing the theory presented in Section 4.4. Prior to the interaction, the vortex has a high swirl velocity. An unbalance in the pressure field occurs during the interaction since the vortex is slowed down by the transfer of energy to the z-direction. This may push the vortex in the experiment to the side walls of the wind tunnel, which causes a stretching of the vortex and transfers energy/momentum to the side walls and is then dissipated. This changes the velocity profile of the experimental vortex as well as the core radius and its strength. The velocity profile of the vortex was measured with the test airfoil removed as the experiment was conducted. As a result, the vortex will most likely not be as strong as that provided in Straus et al. (1990) at the start of the interaction which may enhance the diffusion of the vortex.

The results presented in Figure 5.21 for the simulation and the discrete vortex method results shall now be discussed. Notice that the lift coefficient obtained through simulation and inviscid method agree from the release location until just after the vortex passes the leading edge of the airfoil (i.e. $(x / c) \in[-0.5,0.75])$. After this point there is a deviation in the lift variation. Since the results obtained by Renzoni (1987) are based on an inviscid panel method, the noticeable differences in the lift variation after $(x / c)=$ 0.75 may be attributed to viscous effects. The inclusion of viscosity allows for the formation of Kármán vortices which are not captured by the discrete vortex method. The 
discrete vortex method does, however, release a vortex at the trailing edge of the airfoil at every time step. The discrete vortex released by the inviscid method does not allow for the formation of Kármán vortices as a result of the methods inability to allow for flow separation (which initiates the shedding of Kármán vortices) which also adds to the differences.

By examining the moment coefficient, shown in Figure 5.22, several conclusions concerning the comparison of data obtained through simulation with experimental results and those from the inviscid panel method simulation. Note that the data for each case has trends which are inverted when compared to the lift coefficient (holds true for each data set illustrated). Therefore, the trends and basic relationships shall be presented and the discussions presented for the comparison of the lift coefficient shall also be referred to for the moment coefficient. Upon comparison, the moment data obtained by simulation is nearly identical to the data obtained from experiment until the vortex is about to reach the mid chord of the airfoil (i.e. $(x / c)=0.4$ ). It is at this point that the experimental data begins to converge asymptotically to the zero value. Note that for the lift coefficient this convergence to zero occurred for at a vortex location of $(x / c)=0.1$. Therefore, the asymptotic nature of the experimental moment coefficient results in a dampening of the vibratory loads on the blades after the experimental vortex has diffused. As can be seen through a comparison of the simulation results with those obtained by Renzoni (1987), the viscous effects appear to have a substantial contribution the moment coefficient as is shown by the differences between the discrete vortex method results and the LES results. The viscous effects with respect to the moment coefficient acting on the airfoil become 
evident at the vortex location of $(x / c)=0.1$. Furthermore, since the drag coefficient data is unavailable, there may be significant differences between the drag data obtained from the LES and those obtained using the discrete vortex method as the discrete vortex method is unable to predict the profile drag at all since it is an inviscid method.

Based on the above discussions it is apparent that LES provides data comparable to that of the inviscid panel method used by Renzoni (1987). The differences between which are seen between the simulation results and those of Straus et al. (1990) may be captured by modeling the core growth using Equation ( 4.17 ), which should be investigated in the future. Despite these differences and employing the above arguments, it may be concluded that the LES is a valid method for AVI analyses, and it shall be used in the studies related to mitigating the airfoil-vortex interaction. 


\section{Chapter 6: Aerodynamics of the Airfoil-Vortex Interaction II: Mitigation of the Airfoil-Vortex Interaction}

In the present work, flow simulations around an assumed two-dimensional flow around a NACA 0012 airfoil were performed using Large Eddy Simulation. Like the validation simulations, a vortex was superimposed onto the domain in such a manner that the vortex axis is parallel to the blade span, as is shown in Figure 1.1 and Figure 2.11. In the present chapter, minimization of the effect of vortex-rotor blade interaction is attempted. The basis of comparison which shall be used involves a comparison of the lift, drag and moment coefficients (which are defined in Section 5.5) for several different geometries. The validated and verified results for Domain A shall be employed as the basis of comparison. Including the original geometry presented in Section 5.2, NACA 0012 airfoils with two different cavities have been simulated to mitigate the resulting unsteady blade airloads due to the blade-vortex interaction. The cavities were placed at the quarter chord location of the airfoil and were each $5 \%$ of the chord in width and spanned from the upper surface to the lower surface of the airfoil. A straight cavity and a cavity at $-45^{\circ}$ from the vertical axis were investigated. Based on the verification analysis presented in Section 5.8, large eddy simulation with the time steps and interpolation schemes outlined in Section 5.1 (i.e. using the Smagorinsky model and the Van Driest 
wall damping model) are used to obtain solutions related to mitigating the interaction. The Lamb-Oseen model shall also be used to superimpose the vortex into the computational domain throughout the mitigation analyses (refer to Equation ( 4.16 ) and Sub-Section 5.4.2). In order to quantify the mitigation of the interaction some discussion is presented in Section 6.4 which relates the spike in lift resulting from the interaction to the noise propagated to an observer. Beyond the original comparison between the aforementioned two cavities and the baseline case using the conditions defined by Straus et al. (1990) (see Section 5.4) (referred to as Mitigation Study (MS) 1), the miss distance will be decreased to the worst case scenario (i.e. $y_{v}=0 \mathrm{~m}$ ). This study shall be performed for Domain A (simulated using the vortex conditions defined by Straus et al. (1990) with the miss distance set to zero) and for the cavity that minimizes unsteady blade-loads based on the simulations using the conditions defined by Straus et al. (1990) (referred to as MS 2). Refer to Section 6.6 for the setup and results of this simulation. Upon completing the miss distance analysis, the freestream velocity will be increased to a Mach number of 0.25 and the miss distance will remain zero.

Refer to Section 5.1 for a discussion of the computational resources employed as well as the governing equations that governed the mitigation study. The computational models (i.e. geometry) will now be presented as they differ from those used in Chapter 5:. The fluid models that are used for the following mitigation study are the same as per the validation study, refer to Section 5.2. 


\subsection{Computational Models}

For the validation and verification study, the LES must be employed to solve for the aerodynamic coefficients during a parallel blade-vortex interaction a quasi threedimensional domain. However, a parallel blade-vortex interaction has been shown to be predominantly a two-dimensional phenomenon (as discussed in Section 2.1) and therefore this requirement by ANSYS CFX 11.0 should not hinder the results obtained in any form. The quasi three-dimensional simulation is established by using a thin threedimensional computational domain and is required by ANSYS CFX 11.0 to obtain a solution of the two-dimensional problem. Using ANSYS ICEM CFD the aforementioned geometries have been created and are shown in Figure 6.1 (straight cavity) and Figure 6.2 ( $45^{\circ}$ cavity).

The chord length of the airfoil remains that as per the validation analysis, i.e. $c=0.46 \mathrm{~m}$. The profile of the airfoil (i.e. its shape) was again selected as a NACA 0012 airfoil. The computational domains based on the geometry presented in Figure 6.1 and Figure 6.2 shall now be presented. 


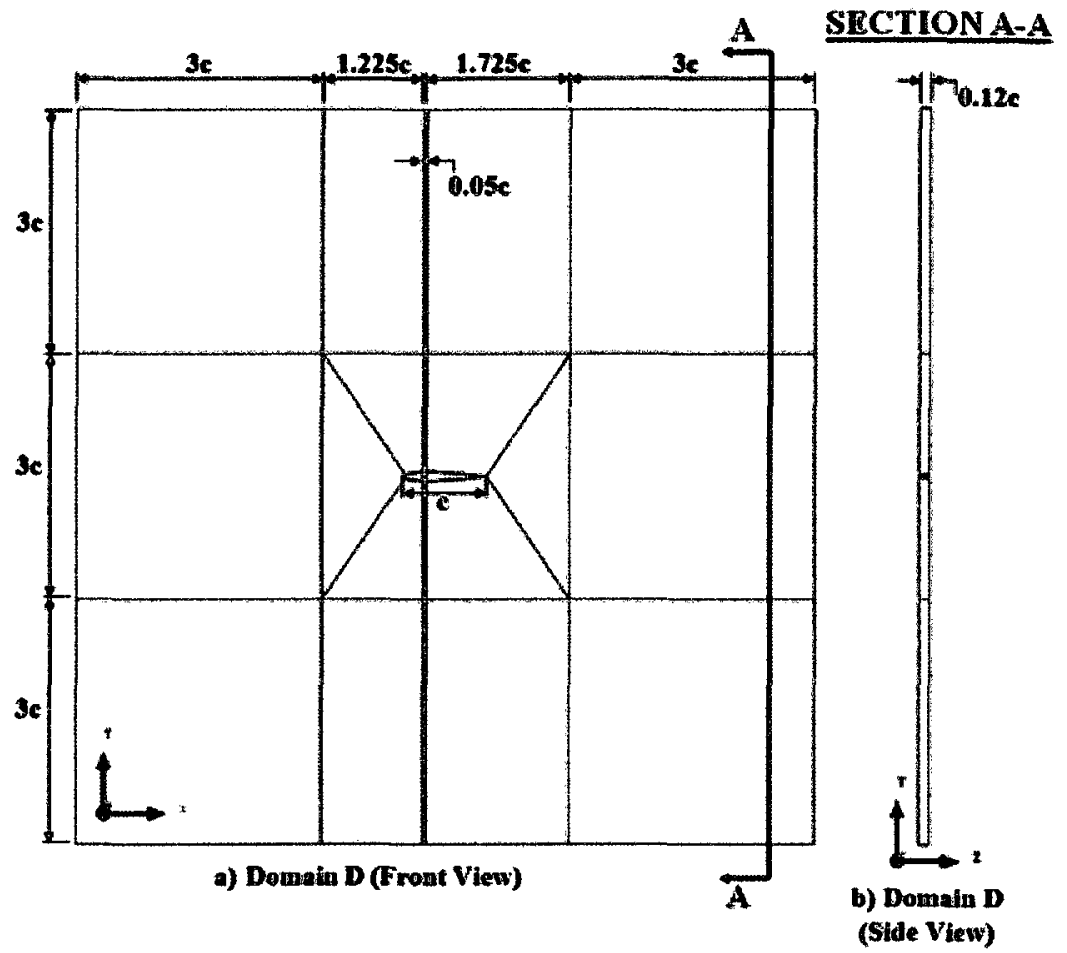

Figure 6.1: NACA 0012 Airfoil with Straight Cavity Computational Model, referred to as Domain D

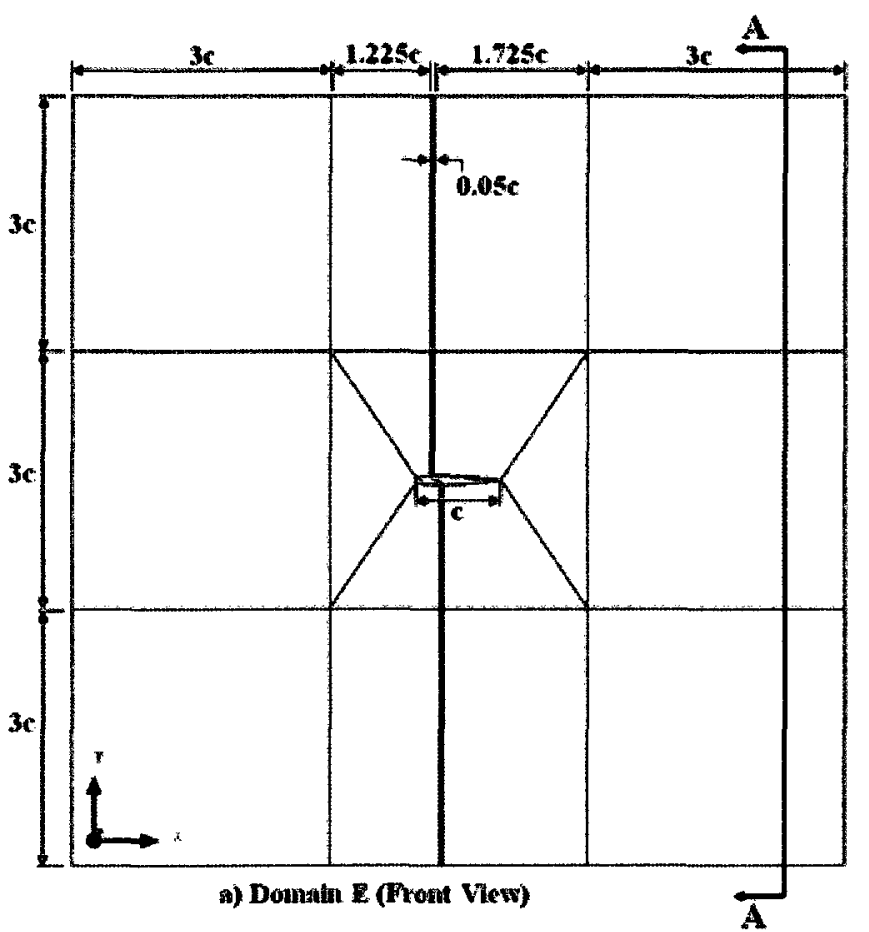

SECTIONA-A

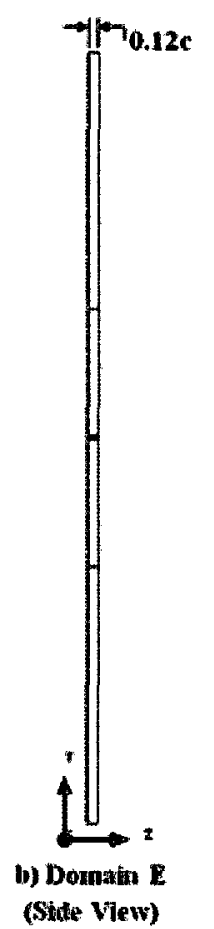

Figure 6.2: NACA 0012 Airfoil with $4^{\circ}$ Cavity Computational Model, referred to as Domain E 


\subsection{Computational Domains}

For computational efficiency reasons, the computational domains based on Figure 6.1 and Figure 6.2 were each divided into 21 blocks. Since the block definition is identical for Domain E and illustrated in Figure 6.3, these blocks are only shown for Domain D.

Note that Figure 6.3 illustrates Domain D. However, despite the cavity being slanted at $45^{\circ}$ in Domain $E$, the block definition for Domain $E$ is identical to that of Domain D.

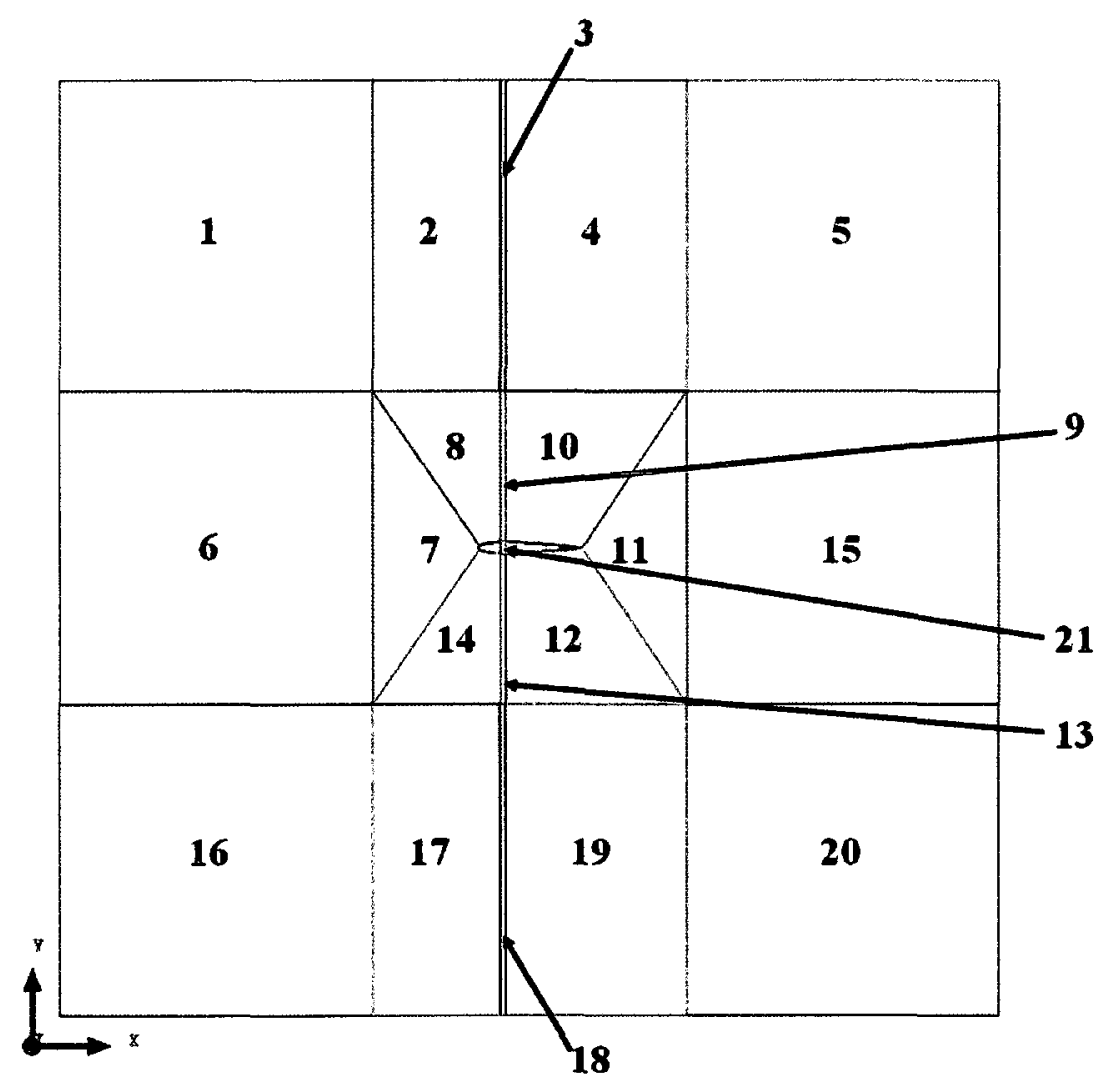

Figure 6.3: Block Definitions in ANSYS ICEM CFD 
Based on the block definition presented in Figure 6.3, the size of the computational grid may be defined for both Domain D and E. Figure 6.4 illustrates the basis for the definition of each mesh for analysis related to the mitigation of the airfoilvortex interaction.

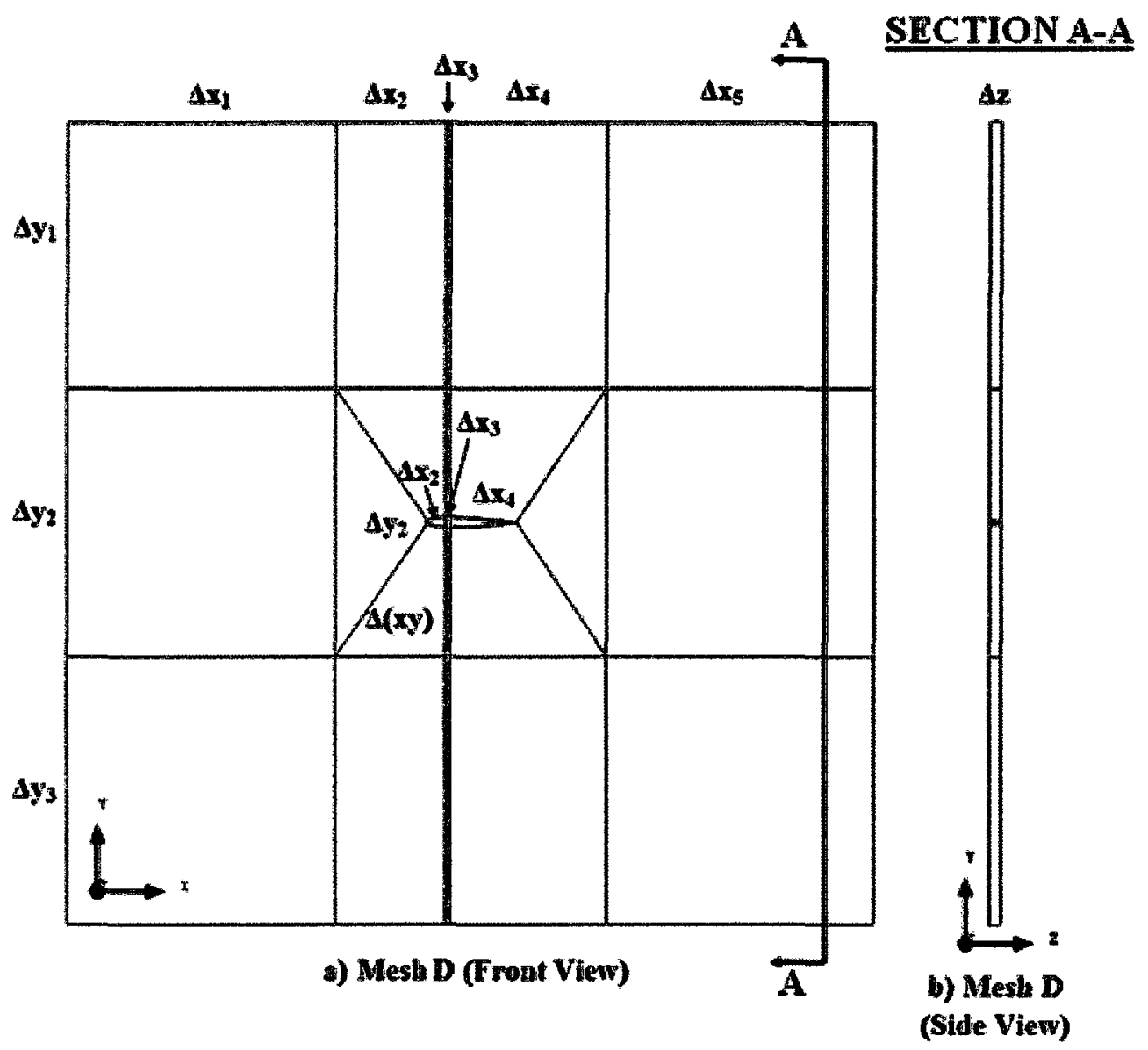

Figure 6.4: Node Definition for the Computational Domains Used in the Mitigation Studies

Defining each of the $(\Delta x, \Delta y, \Delta z)_{i}$ parameters as well as $\Delta(x y)$ results in two separate computational domains, which as shall be referred to as Domains D and E. As per the validation analysis, these parameters represent the number of nodes associated with each block defined in Figure 6.3. Table 6.1 represents the definitions of the number 
of nodes associated with the values defined in Figure 6.4 including the total number of nodes associated with each domain.

\begin{tabular}{|c|c|c|c|c|c|c|c|c|c|c|c|}
\hline Domain & $\Delta \mathbf{x}_{1}$ & $\Delta \mathbf{x}_{2}$ & $\Delta \mathbf{x}_{3}$ & $\Delta \mathbf{x}_{4}$ & $\Delta \mathbf{x}_{5}$ & $\Delta y_{1}$ & $\Delta y_{2}$ & $\Delta y_{3}$ & $\Delta x y$ & $\Delta \mathbf{z}$ & Total \\
\hline $\mathrm{D}$ & 64 & 70 & 30 & 110 & 90 & 64 & 180 & 64 & 180 & 4 & 868,080 \\
\hline$E$ & 64 & 70 & 30 & 110 & 64 & 64 & 180 & 64 & 250 & 4 & 836,256 \\
\hline
\end{tabular}

Table 6.1: Node Definitions Resulting in the Computational Domains Used for the Mitigation Study

For $(\Delta x, \Delta y, \Delta z)_{i}$ a uniform spacing was employed in each computational domain and for $\Delta(x y)$ the nodes were clustered around the airfoil wall with an expansion ratio of 1.05 . For the initial study related to mitigating the interaction, the results presented in Chapter 5: for Domain A shall be compared with results for the same boundary conditions (refer to Section 5.4) applied to Domains D and E. After this several parameters shall be varied (namely the freestream velocity and the miss distance) using the cavity which provides the most benefits with respect to minimization of the change in the blades airloads. The resulting multi-block structured grids for the airfoil centric computational domains are illustrated in Figure 6.5 (straight cavity) and Figure $6.6\left(45^{\circ}\right.$ cavity). The side view is omitted for each domain as they are the same as those shown by Figure 5.4.

For each computational domain presented a time step of $1 \times 10^{-6}$ seconds was employed for the simulations performed based on the conditions defined in Section 5.4 as well as for the zero miss distance simulations. For the Mach 0.25 case a time step of $1 \times 10^{-7}$ seconds has been employed. Again, as the validation of the computational method, these time steps were selected to ensure accuracy. They are also required as a result of the selection of an explicit time-marching scheme as well as the temporal resolution requirement of LES; the Courant-Friedrichs-Lewy (CFL) number must be less than unity 
for sufficient temporal resolution. In the present analysis, it was ensured that the CFL was below unity throughout each domain and the entire simulation. The Smagorinsky constant $C_{s}=0.1$ was set to ensure that no numerical dissipation of the vortex as it traverses the computational domain. Upon creating the computational domains, one must next set the necessary boundary conditions and initialize the domain in a proper manner. These details show now be addressed.

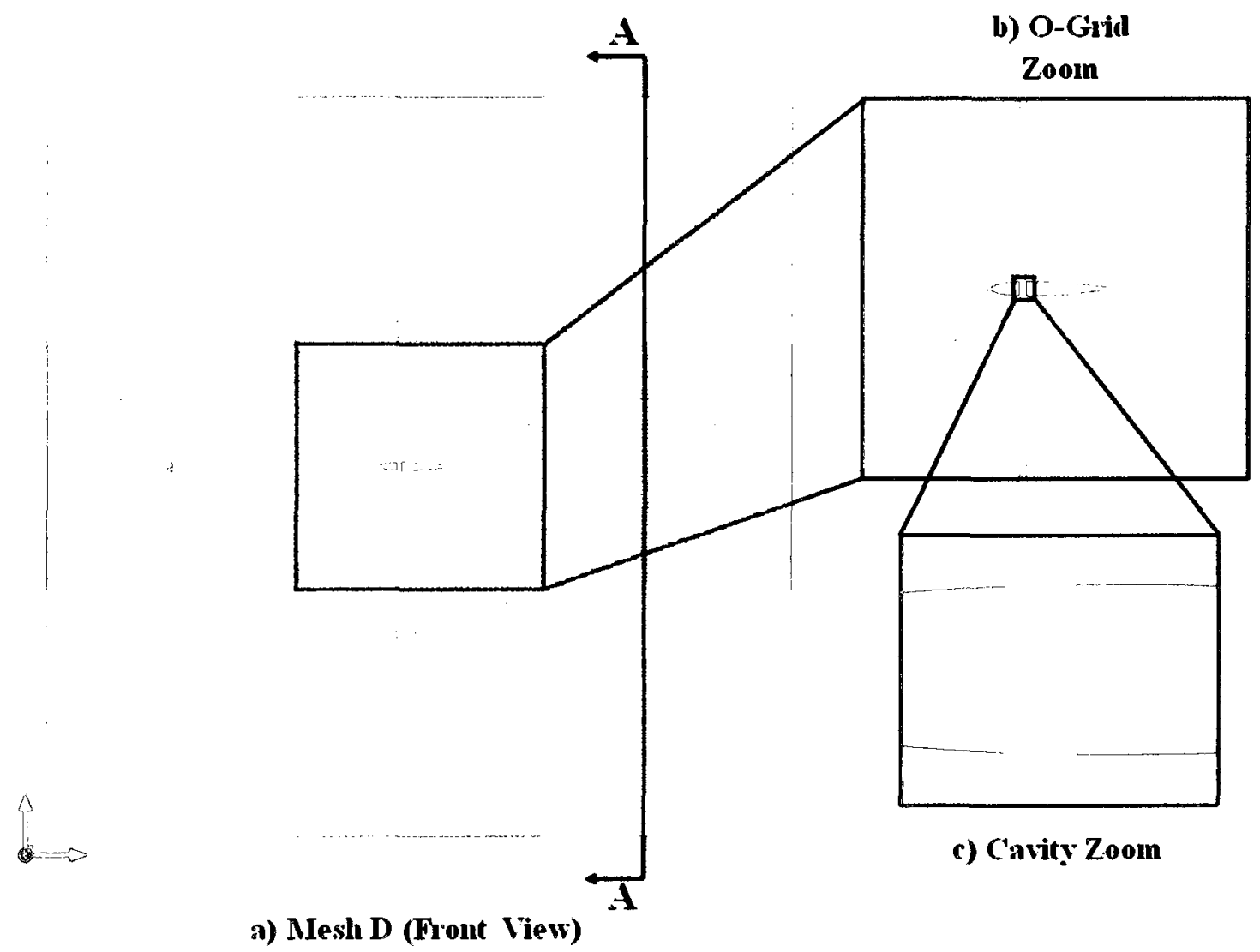

Figure 6.5: Computational Grid for Domain D 


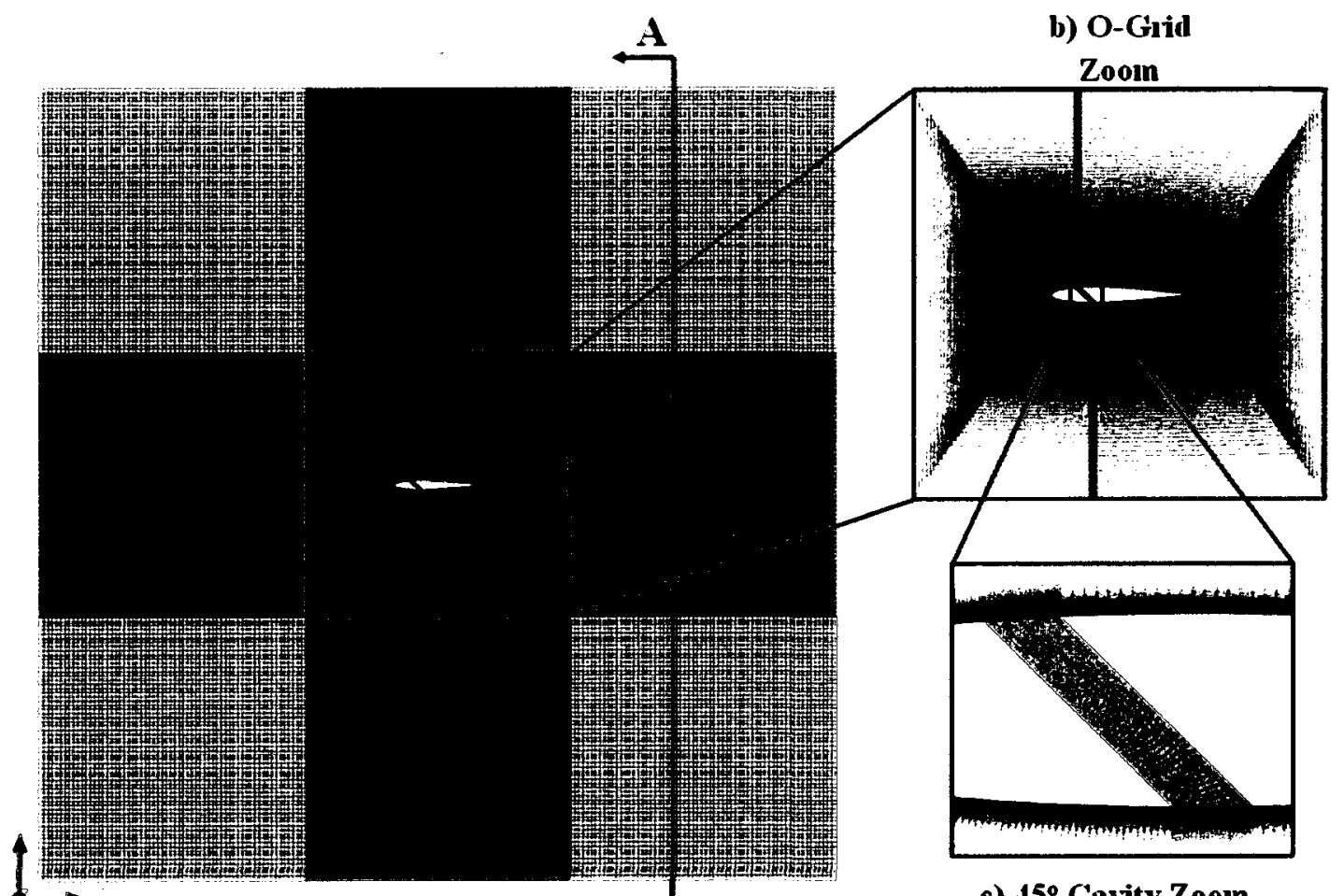

a) Mesh E (Front View)

c) $45^{\circ}$ Cavity Zoom

Figure 6.6: Computational Grid for Domain $E$

\subsection{Boundary Conditions and Vortex Initialization}

For each of the aforementioned mitigation studies, the vortex parameters as well as the boundary conditions imposed on the computational domains differ slightly. As a result, each mitigation study shall now be detailed in full. The parameters define the vortices using the same sequence of steps outlined in Sub-Section 5.4.2, shall be presented first. The boundary conditions which have been imposed on the computational domains shall follow.

\subsubsection{Initialization of the Vortices for the Mitigation Studies}

For the mitigation studies, the model of the vortex for the validation and verification analysis shall be employed (see Sub-Section 5.4.2 in particular Equation 
( 5.1 ) through Equation ( 5.5 )). Furthermore, as with the validation study, a unit step function is also imposed on the vortex for the same reasons presented in Sub-Section 5.4.2. For the vortex, the defining parameters are the vortex strength, $\Gamma$, the core radius, $r_{c}$, the release location of the vortex with respect to the leading edge of the airfoil, $x_{v}$, the vertical miss distance, $y_{v}$, and the freestream velocity, $U_{\infty}$. Table 6.2 outlines these parameters for the aforementioned mitigation studies.

\begin{tabular}{|c|c|c|c|}
\cline { 2 - 4 } \multicolumn{1}{c|}{} & \multicolumn{3}{c|}{ Mitigation Study } \\
\cline { 2 - 4 } \multicolumn{1}{c|}{} & $\mathbf{1}$ & $\mathbf{2}$ & $\mathbf{3}$ \\
\hline $\boldsymbol{U}_{\infty}[\mathrm{m} / \mathrm{s}]$ & 12.2 & 12.2 & 85.075 \\
\hline $\boldsymbol{r}\left[\mathrm{m}^{2} / \mathbf{s}\right]$ & 0.89792 & 0.89792 & 6.26152 \\
\hline $\boldsymbol{r}_{\boldsymbol{c}}[\mathrm{m}]$ & 0.02325 & 0.02325 & 0.02325 \\
\hline $\boldsymbol{x}_{v}[\mathrm{~m}]$ & -0.23 & -0.23 & -0.23 \\
\hline$y_{v}[\mathrm{~m}]$ & -0.06704325 & 0 & 0 \\
\hline
\end{tabular}

Table 6.2: Defining Vortex Parameters for the Mitigation Studies

Regardless of the freestream velocity, the non-dimensional velocity profile presented in Figure 5.8 was maintained as the non-dimensional velocity profile of the vortex throughout the mitigation studies. Note that the computational domains for the mitigation study differ from that of Domain A (refer to Figure 5.4, Figure 6.5 and Figure 6.6 to visualize the differences). The boundary conditions imposed on Domain D and E will also differ from that of Domain A. Furthermore, the freestream velocity is altered for mitigation study 3 . As a result, the differences in the boundary conditions for this study must also be identified and outlined. 


\subsubsection{Boundary Conditions Imposed on the Computational Domains}

Before presenting the boundary conditions for the computational domains, some nomenclature for the boundary conditions must be presented. Figure 6.7 presents Domain D with the associated boundaries indicated (for the same reasons mentioned in Section 6.2 Domain E shall not be illustrated). Refer to Figure 5.9 for the boundary definition for Domain A.

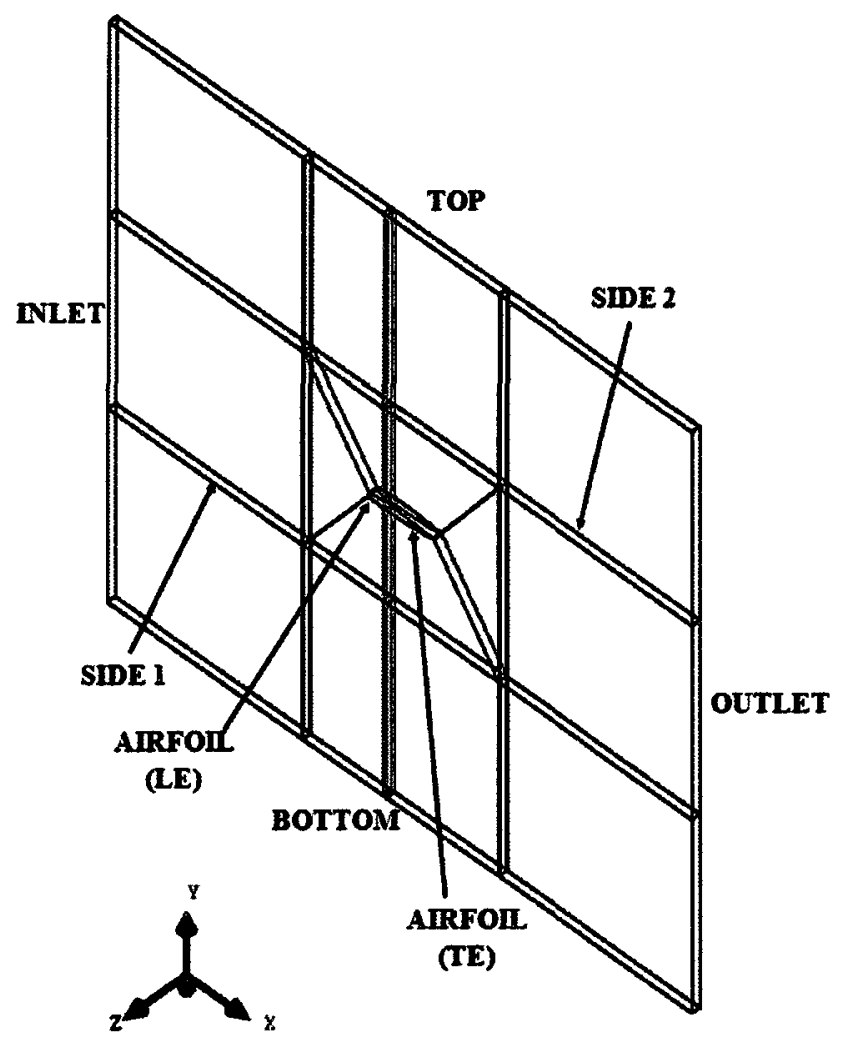

Figure 6.7: Boundaries for the Computational Domains D and E

Based on the boundary definitions presented in Figure 6.7 as well as the data presented in Table 6.2 , the boundary conditions may now be provided. To being with the boundary conditions shall be presented for mitigation study 1 . 
In mitigation study 1 and 2, the parameters of the vortex are outlined in Table 6.2. Furthermore, the boundary conditions on Domain A remain the same as those presented in Table 5.5. The boundary conditions for both Domain D and E are presented in Table 6.3 for the first two mitigation studies. Note that the only difference between mitigation study 1 and 2 is that the miss distance is decreased to analyze the case of a fully parallel airfoil-vortex interaction, i.e. $y_{v}=0 \mathrm{~m}$.

\begin{tabular}{|c||c|c|}
\hline Boundary & Boundary Details & Value \\
\hline INLET & Speed Normal to Inlet & $12.2 \mathrm{~m} / \mathrm{s}$ \\
\hline OUTLET & Average Static Pressure & $1 \mathrm{~atm}$ \\
\hline AIRFOIL (LE) & Smooth Wall with No Slip & - \\
\hline AIRFOIL (TE) & Smooth Wall with No Slip & - \\
\hline TOP & Free Slip & - \\
\hline BOTTOM & Free Slip & - \\
\hline SIDE 1 & Symmetry & - \\
\hline SIDE 2 & Symmetry & - \\
\hline
\end{tabular}

Table 6.3: Boundary Conditions Used for the Domain D and E for MS 1 and MS 2

In mitigation study 3, the vortex parameters used are detailed in Table 6.3. The boundary conditions in Figure 6.7 (for Domain D and E) and Figure 5.9 (for Domain A) remain the same for the every boundary with the exception of the inlet (see Table 6.3 and Table 5.5 for full boundary definitions beyond this). Regardless of the domain (i.e. with or without a cavity), the inlet boundary condition for mitigation study 3 is identical and is outlined in Table 6.4 .

\begin{tabular}{|c||c|c|}
\hline Boundary & Boundary Details & Value \\
\hline INLET & Speed Normal to Inlet & $85.075 \mathrm{~m} / \mathrm{s}$ \\
\hline
\end{tabular}

Table 6.4: Inlet Boundary Condition for all Domains for Mitigation Study 3 
Employing the boundary conditions outlined for mitigation study 1 (see Table 6.2 and Table 6.3), the results of the LES simulations may now be presented.

\subsection{Quantifying the Mitigation of the Airfoil-Vortex Interaction}

Some relations must first be defined and some definitions must be provided to quantify the mitigation achieved with the use of such novel cavities (or passages). Note the addition of the term, $\Delta C_{l}$, which is defined as the incremental lift coefficient in Figure 6.8. The incremental lift coefficient is the difference between the local maximum and absolute minimum of the lift coefficient, $C_{l}$, (as indicated in Figure 6.8 ) caused by the interaction between a tip vortex and an airfoil as the vortex passes the airfoils leading edge.

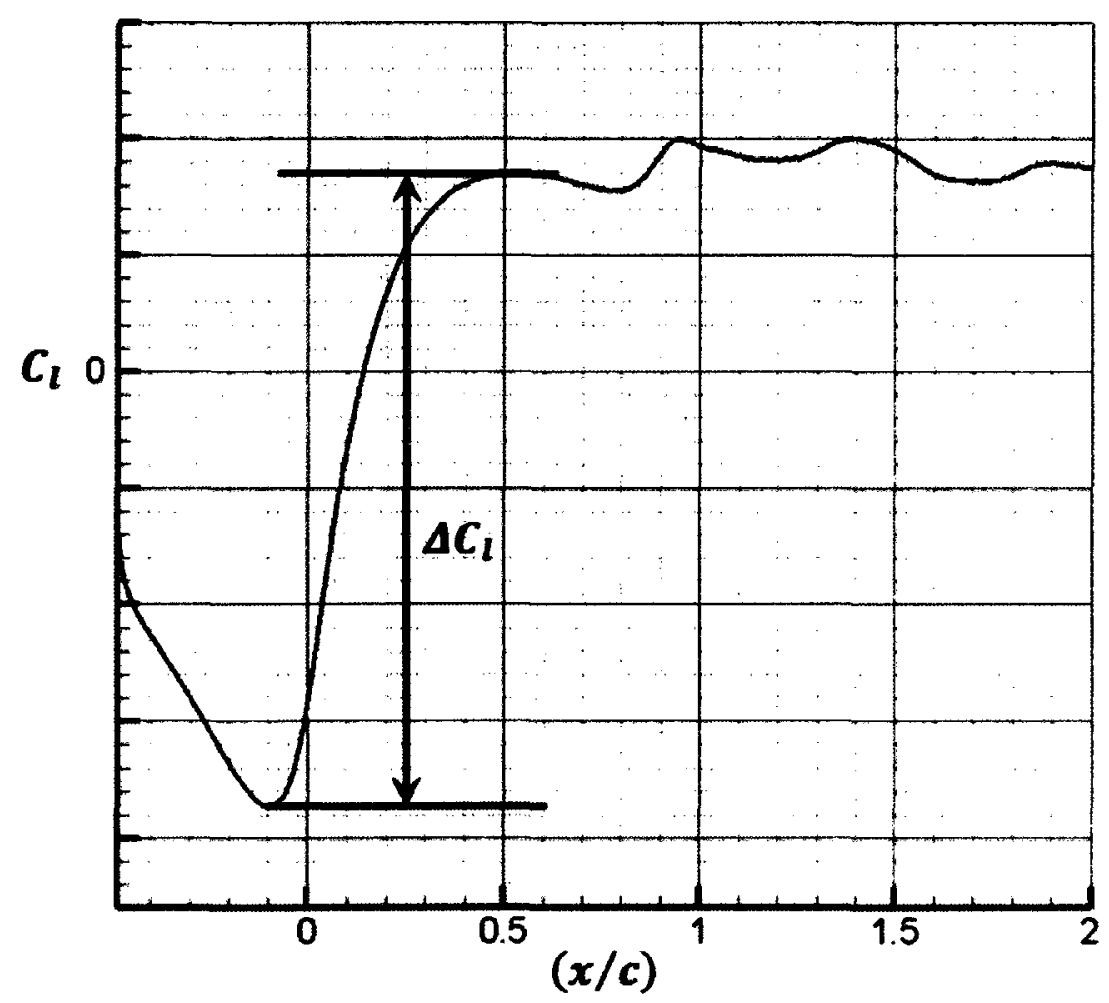

Figure 6.8: Incremental Lift Coefficient Definition 
Renzoni (1987) provided a correlation between the incremental lift coefficient and the characteristics of the vortex such as convection speed, $U_{\infty}$, miss distance, $y_{v}$, and the strength of the vortex, $\Gamma$ ). Renzoni (1987)'s correlation is given as:

$$
\Delta C_{l}=0.7 \frac{\Gamma}{U_{\infty} c \sqrt{y_{v} / c}}
$$

Also, Leverton and Taylor (1966) found that the sound pressure propagated to an observer, $p$, was proportional to the square of the vortex strength, i.e. $\Gamma^{2}$, and the $4^{\text {th }}$ power of the tip speed of a rotor blade, i.e. $(\Omega R)^{4}$. Because the current investigation is a two-dimensional study, the tip speed of the rotor blade is taken as the freestream velocity (i.e. $\left.(\Omega R)=U_{\infty}\right)$. Therefore, the sound pressure propagated to an observer was determined to be

$$
p^{2} \propto U_{\infty}^{4} \Gamma^{2}
$$

Through some manipulations of Equations ( 6.1 ) and ( 6.2 ) it can be shown that

$$
p \propto \Delta C_{l}
$$

Equation ( 6.3 ) indicates a linear proportionality between the incremental lift coefficient and the sound pressure propagated to an observer. Therefore, if the incremental lift coefficient is decreased by $10 \%$ there is an equal decrease in the sound pressure propagated to an observer on account of the novel cavity. Through the implementation of a cavity, the interaction obtains a certain reduction in the incremental lift coefficient. Hence, Equation ( 6.3 ) can be used to quantify the mitigation of the interaction with regards to reduction of the sound level propagated to an observer. For further discussion 
regarding the inherent link between the fluctuating airloads and the sound pressure level refer to Chapter 2..

\subsection{Mitigation Study 1}

In performing mitigation study 1 , three different geometries shall be simulated for the aforementioned boundary conditions and vortex parameters. As mentioned previously, the geometries compared are a NACA 0012 airfoil with no adaptation, the same airfoil profile with a vertical cavity and the same airfoil shape with an inclined cavity $\left(-45^{\circ}\right.$ from the vertical) and are presented in Section 6.1 (cavities) and Section 5.2 (no cavity). Each cavity has a width of $5 \%$ of the airfoils chord (i.e. $0.023 \mathrm{~m}$ ) centered about the $1 / 4$ chord location of the airfoil (i.e. $0.115 \mathrm{~m}$ ). These geometries were meshed using structured grids, as outlined in Section 6.2 (cavities) and Section 5.3 (no cavity). To simplify the discussion, Domain A refers to the geometry/mesh with no cavity, Domain D refers to the geometry/mesh with the straight cavity and Domain E refers to the geometry/mesh with the $45^{\circ}$ cavity. Refer to Section 5.6 for a discussion regarding the structure of Domain A. Domain D employed a similar mesh structure to Domain A (again, refer to Section 5.6) in the sense that nodes were added in the blocks downstream of the airfoil in attempt to capture the interaction of the vortex with the wake. For both Domain $\mathrm{D}$ and $\mathrm{E}$, the O-grid block was broken down into 5 additional blocks when compared to Domain A (for a comparison refer to Figure 5.2 and Figure 6.3 to visualize the added blocks). The addition of these blocks allowed for the grid resolution to be significantly higher in the cavities themselves in order to resolve the boundary layer in the cavities themselves, while still clustering nodes around the airfoil as necessary to 
obtain accurate results. Figure $6.5 \mathrm{c}$ and Figure $6.6 \mathrm{c}$ present a blown up image of the mesh in the cavities. By referring to Table 6.1 as well as Figure 6.4 the node definitions may be realized. It can be seen that Domain D contains 868,080 nodes and Domain E contains 836,256 nodes. Note that Domain A only contains 760,464 nodes. Therefore the additional nodes in Domains $\mathrm{D}$ and $\mathrm{E}$ are all added to improve the resolution of the results obtained through the cavities whereas the rest of the node definitions for Domains $\mathrm{A}, \mathrm{D}$ and $\mathrm{E}$ remain nearly the same (with the aforementioned exception for Domain $\mathrm{D}$ ).

To begin with the velocity and pressure fields present during the airfoil-vortex interactions shall be presented to better understand the differences in the flow physics present for Domains A, D and E. Figure 6.9 and Figure 6.10 represent the velocity and pressure fields respectively. These figures present the flow field for numerous locations of the vortex beginning from its release location (i.e. $1 / 2$ chord upstream of the airfoils leading edge) until it is $1 / 2$ chord downstream of the airfoils trailing edge. 5 different instances of time are also illustrated as separate clockwise rotating vortices travel downstream. 
Velocity $\left[m s^{\wedge}-1\right]$
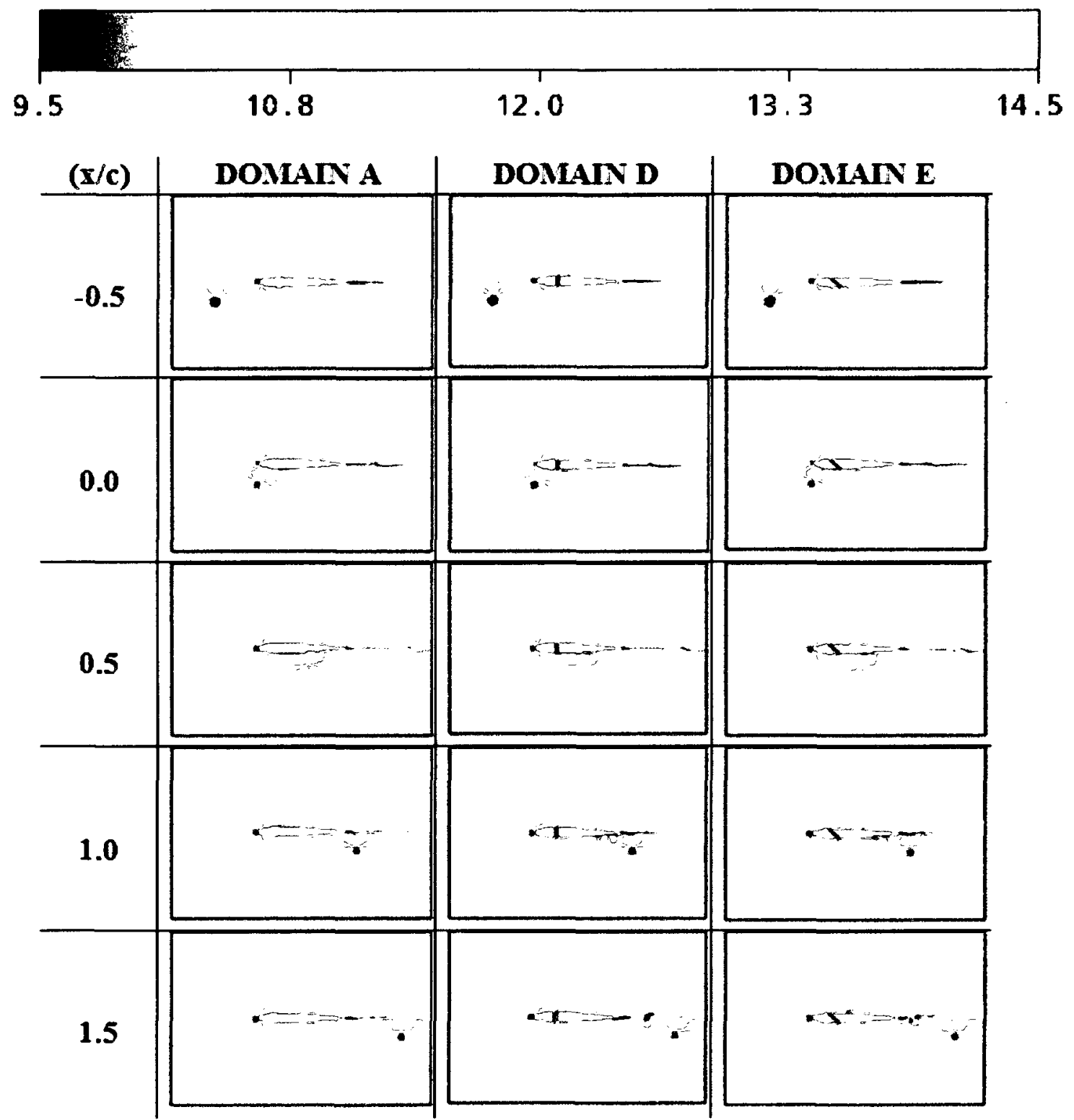

Figure 6.9: Velocity Fields for the Indicated Vortex Locations for Mitigation Study 1 

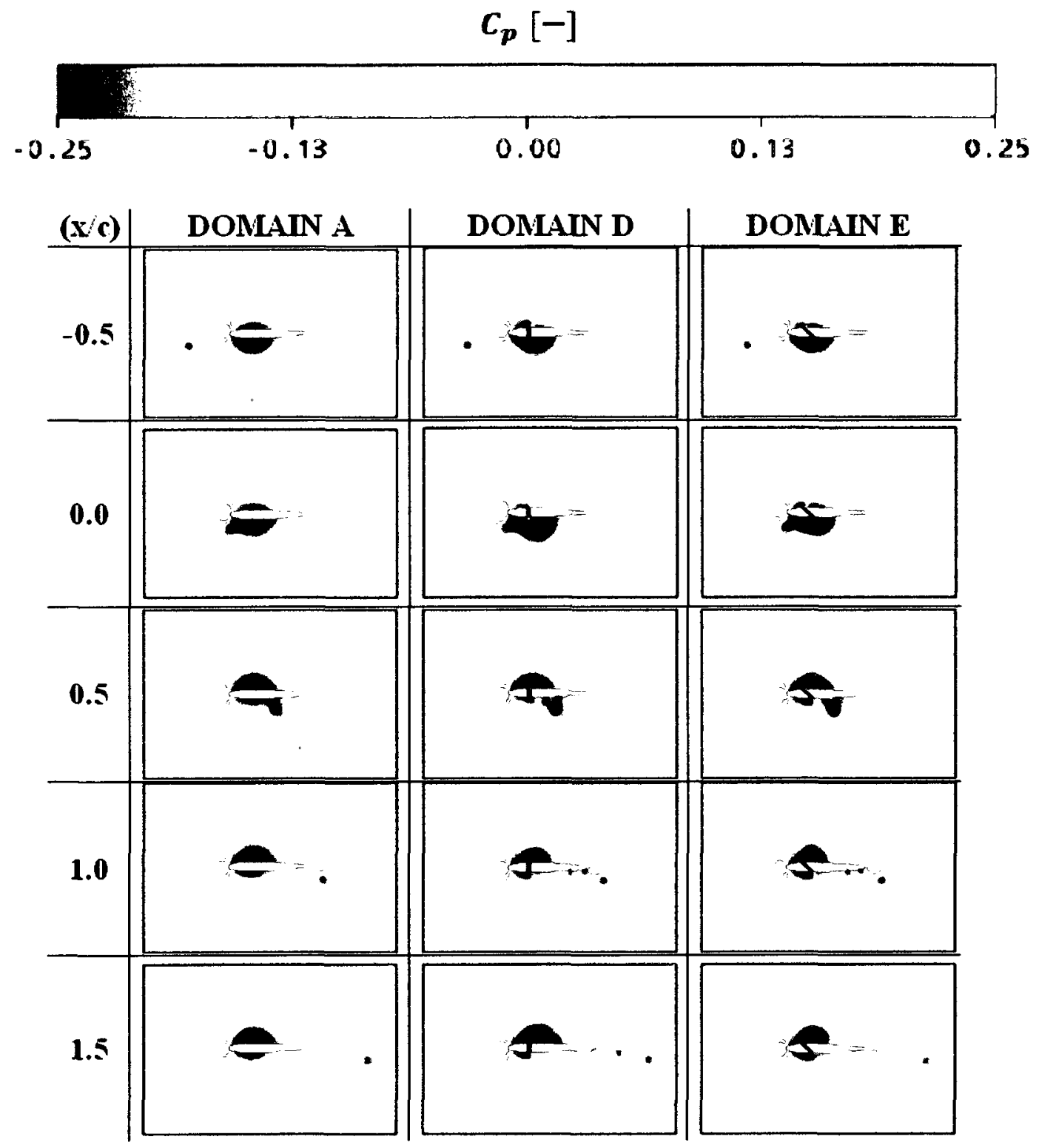

Figure 6.10: Pressure Fields for the Indicated Vortex Locations for Mitigation Study 1

The time histories of the velocity field and the pressure field provide good insight regarding the flow physics of an airfoil-vortex interaction. The airfoil-vortex interaction can be described in three different phases. At the beginning of AVI, the vortex is upstream of the airfoil and moves towards the leading edge (i.e. $(x / c)=-0.5$ to 
$(x / c) \approx 0)$ as is illustrated in Figure 6.9 and Figure 6.10 for Domain A. Throughout this phase, the vortex induces a downwash on the airfoil. As a result, the leading-edge stagnation point to move along the upper surface of the airfoil. This causes an increase in pressure over the airfoils upper surface. The second phase begins after the vortex passes the leading edge of the airfoil (i.e. $(x / c)=0$ to $(x / c)=1$ ), which induces an upwash on the airfoil. Consequently, the stagnation point moves from the upper surface to the leading edge. Finally, as the vortex convects further past the leading edge the stagnation point moves towards the lower surface. The movement of the stagnation point affects the blades loading, particularly the rate of change of lift which is proportional to the sound pressure level which is propagated to an observer. As the AVI progresses, the vortex is distorted while traveling the chord length of the airfoil. In the third phase, the vortex leaves the airfoil (i.e. $(x / c)>1$ ) which generates wake perturbations. The Kármán vortices shed by the airfoil during the interaction result in varying inductions of upwash/downwash, which results in small fluctuations of the airloads due to the tip vortex and Kármán vortices interacting with each other and the airfoil surface as well.

In order to determine which airfoil mitigates the AVI the best, a similar discussion shall be provided for Domains $\mathrm{D}$ and $\mathrm{E}$ with emphasis on the significant differences. The velocity and pressure fields present for Domain D (refer to Figure 6.9 and Figure 6.10) shall be detailed in full using the same phase descriptions as presented for Domain A. At the beginning of AVI, the vortex is upstream of the airfoil and moves towards the leading edge (i.e. $(x / c)=-0.5$ to $(x / c) \approx 0$ ). This phase of AVI has the same flow physics as Domain A with the exception of some of the vortex flow to flow through the cavity, 
thereby lowering the velocity on the upper surface and slightly increases the pressure over this surface as well. In the second phase of the interaction, the upwash induced on the airfoil surface is counteracted by flow coming downwards through the cavity. This flow is accelerated towards the wall near the trailing edge segment of the airfoil which results in a counteraction of the upwash induced by the vortex. This results in fluctuations of the blades airloads, in particular with respect to lift. When the vortex is at the leading edge, the boundary layer of the trailing edge segment also thickens on the lower surface of the trailing edge airfoil segment which results in a significant decrease in drag. Furthermore at this location, there is a region of high pressure on the upper edge of the wall of the trailing edge segment and a vortical type structure in the midpoint of the two walls near the lower surface. This also contributes to the significant fluctuations of the blades airloading. As the vortex reaches the mid chord of the airfoil, vortical structures are now convecting downstream from the lower surface of the wall found on the trailing edge segment of the airfoil. This convection causes a further increase in the unsteady airloads experienced throughout the airfoil. The pressure field on the lower surface is segmented by a region of higher pressure on the surface between the tip vortex and the cavity as well. As a result of the thickening of the boundary layer, a shear layer is also present at this vortex location. This further thickens the boundary layer and results in an increase in drag along with the other aerodynamic loads present. As the vortex approaches the trailing edge, several vortical structures shed from the cavities lower wall may be seen. This further induces an upwash on the airfoils surface creates significantly unsteady loads on the blades surface. Furthermore, the shear layer convects along the 
airfoils lower surface with the vortex, which causes further fluctuations of the aerodynamic loads. When the vortex is at the trailing edge, the pressure field illustrates in detail the vortical structures shed from the cavities lower wall. In the final phase of the AVI, the tip vortex and the further shed vortices can be seen to interact in a destructive fashion with the airfoils wake. This perturbs the wake in a tremendous manner, which results in further fluctuations of the blade loading. In particular, three vortical structures may be seen interacting with the wake when the vortex is a half chord downstream of the airfoils trailing edge.

As with the discussion presented for Domain D, the velocity and pressure fields for Domain E (refer to Figure 6.9 and Figure 6.10) shall be detailed in full using the same phase descriptions as presented for Domain A. The first phase of the AVI is the same as that of Domain A. The boundary layer on the upper surface of the leading edge segment is pushed downwards through the cavity by the tip vortex, since the vortex is at the leading edge of the airfoil with the $45^{\circ}$ cavity. This results in some alleviation of the drag force. However, this allows a stagnation point to form on the upper surface wall of the trailing edge segment, which causes the flow near the wall to accelerate and a jet type flow to exit near the lower surface of the cavity. The jet interacts with the boundary layer forming a shear layer and thickening the boundary layer which results in the drag over the airfoils surface increasing. The pressure field illustrates a vortical structure moving along the wall of the leading edge segment of the airfoil. When the vortex is at the mid chord of the blade, the stagnation point on the cavity wall of the trailing edge segment has moved from the upper surface to the lower surface and a high pressure region can be 
found between the cavity and the vortex (similar to Domain D). Similar to Domain D, the vortex interacts with the boundary layer creating a shear layer which alters the velocity and pressure fields over the airfoils surface from Domain A. As the vortex approaches the airfoils trailing edge, vortical structures are also shed from the lower surface of the cavity wall as per the Domain D discussion. Furthermore, the upwash induced from these vortices has changed the direction of the jet to upwards through the cavity, which thickens the boundary layer on the upper surface of the trailing edge segment of the airfoil and creates a shear layer between the flow accelerated over the upper surface and the jet through the cavity. This effectively increases the drag on the airfoil. In the final phase of the AVI, these (i.e. in the Domain D discussion) vortical structures perturb the wake again. For Domain E, however, this wake/vortex interaction is much less significant than for Domain D, but is more significant than in Domain A. This implies that the fluctuations of the airloads shall occur for a longer period of time for the cavity than that of Domain A.

Based on the discussion regarding the physics of the interaction for Domains A, $\mathrm{D}$, and $\mathrm{E}$, the aerodynamic coefficients, $C_{l}, C_{d}$ and $C_{m}$, (refer to Figure 6.11 through Figure 6.13 respectively) shall be used to carry on the analysis. The geometry which presents the most benefits in terms of mitigating the airfoil-vortex interaction is selected, based on the discussion following Figure 6.11 through Figure 6.13. Figure 6.11 illustrates a comparison of the lift coefficient, $C_{l}$, as the vortex traverses from a non-dimensional upstream horizontal location of roughly half a chord from the leading edge (i.e. $(x / c)=$ 
-0.5 ) until the vortex is one chord downstream of the trailing edge of the airfoil (i.e. $(x / c)=2.0)$

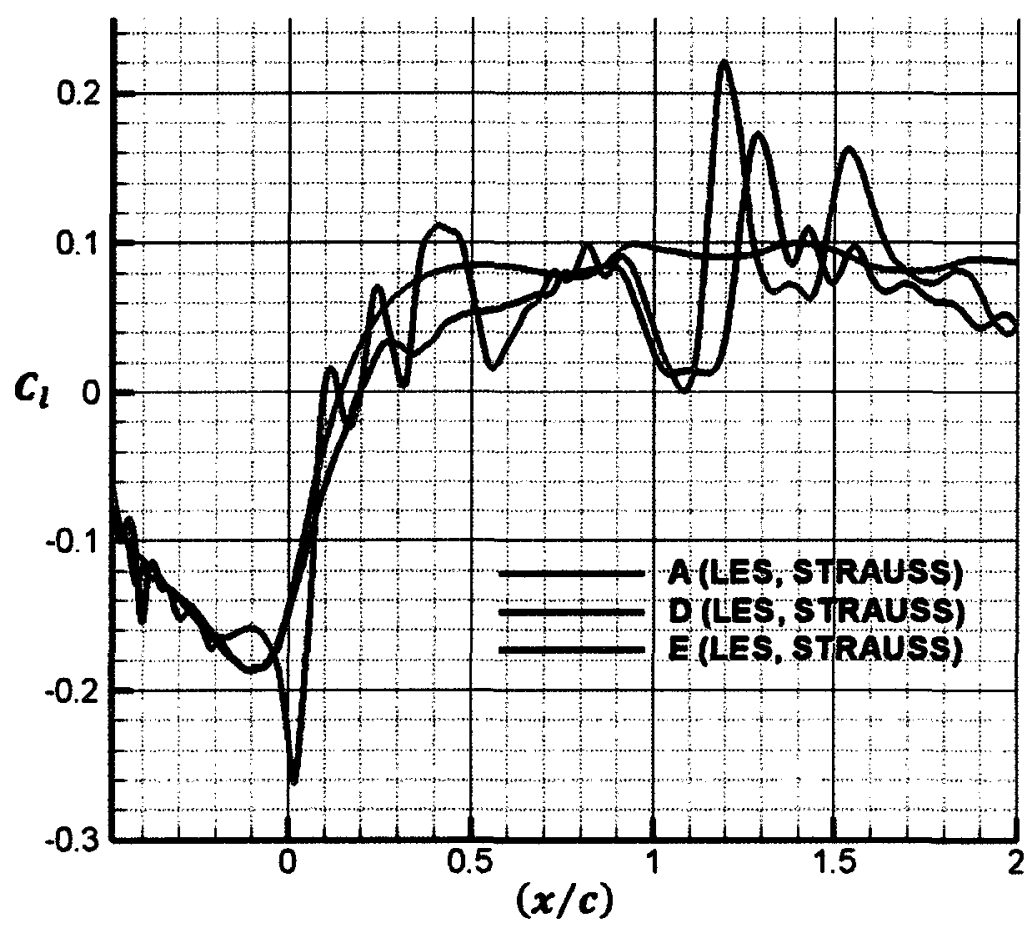

Figure 6.11: Lift Coefficient for Varying Vortex Location for Mitigation Study 1 (i.e. Domains A, D and E)

Figure 6.11 illustrates the temporal variation of the lift coefficient for Domains $A$, $\mathrm{D}$ and $\mathrm{E}$. The lift coefficient is influenced by the pressure distribution over the airfoils surface. Figure 6.11 shows that there is a continuous decrease in lift throughout the first phase of the interaction until a minimum value is achieved for each domain (lowest obtained by Domain D). This is followed by a sudden jump as the vortex core passes the airfoils leading edge. A sudden change in the pressure distribution over the airfoil causes this jump and is associated with the BVI noise which is propagated to an observer as the vortex encounters the airfoil. Despite the lift increase, the magnitude of the maximum lift during the interaction is smaller than the magnitude of the minimum. This difference in 
the maximum and minimum magnitudes is a result of the vortex core distortion as the vortex encounters the airfoil. When the residual component of the distorted vortex is far downstream from the airfoil, the lift returns to its zero value and the flow field in the vicinity of the airfoil will also return to its undisturbed state (not shown in Figure 6.11). Although the amplitudes at the beginning of the interaction are quite large, the fluctuations shown after the vortex leaves the trailing edge of the airfoil result from the wake-vortex interactions (includes the tip vortex and the Kármán vortices). For Domain $\mathrm{D}$, as the vortex convects from its initial upstream location to the leading edge of the airfoil, there a steady decrease in lift. Then, there is a sudden slight increase followed by a decrease of much larger amplitude than that experienced by Domain A. Reasons for this sudden fluctuation were mentioned above in the velocity and pressure fields discussion. In the second phase of the interaction, the mean variation in lift almost follows that of Domain A. However, there are large fluctuations around the mean lift line on account of the aforementioned flow phenomenon (see discussions for Figure 6.9 and Figure 6.10). As the vortex enters its final phase of interaction, there is a drop in lift to zero followed by a sudden increase in lift. This drop is a result of the sudden upwash induced by the shed and tip vortices as they continue to interact with the airfoil thus altering the pressure distribution over its surface. The straight cavity increases vibrations as well as an increase in the noise propagated as the vortex passes the airfoils leading edge (since the incremental lift coefficient is increased relative to that of Domain A). As in Domain A, as the vortex travels from its initial upstream location in Domain $\mathrm{E}$ to the leading edge of the airfoil, a continuous decrease in lift is experienced. As the vortex enters its second 
phase of the interaction for Domain E; an increase in lift until a local maximum is encountered. The increase in lift is lower here than in Domain A: this implies that the BVI noise propagated during the event is lower (as per the discussion in Section 6.4). For the benchmark airfoil, the incremental lift coefficient during the interaction (as defined in Section 6.4) was found to be 0.272 , whereas for the aforementioned flow conditions using the $45^{\circ}$ cavity, the incremental lift coefficient is given as 0.239 . The percent reduction of the incremental lift coefficient achieved through implementing the $45^{\circ}$ cavity when compared to the benchmark airfoil was found to be $3.29 \%$ for a freestream Mach number of 0.036 , a vortex strength of $0.9 \mathrm{~m}^{2} / \mathrm{s}$ and a vertical miss distance of $-0.067 \mathrm{~m}$. Based on Equation ( 6.3 ) and the discussion presented in Section 6.4, this leads to a $3.29 \%$ decrease in the sound pressure which is propagated to an observer (i.e. the interaction from an acoustics standpoint has been mitigated by $3.29 \%$ ). As the vortex enters its final phase of AVI, there is a decrease in lift to a near zero value well before that experienced by Domain A. This is followed by a second increase in lift until a maximum value is reached. The second spike may be attributed to the additional upwash from the vortical structures that are shed from the cavity wall itself which then interact with the airfoil via a secondary interaction. This secondary spike in lift is undesired as it results in a delayed but additional impulsive noise which is propagated to an observer. It is followed by a decrease in lift with a more significant rate of decay (until the asymptotic zero value) then that produced by Domain A. Reasons for these observations may be found in the velocity and pressure field discussions above. 
In a similar manner to Figure 6.11, Figure 6.12 presents a comparison between Domains $\mathrm{A}, \mathrm{D}$ and $\mathrm{E}$ for the drag coefficient for the same range of vortex locations. Figure 6.12 illustrates the temporal variation of the drag coefficient for Domains $A, D$ and $\mathrm{E}$. For Domain A, as the vortex approaches the airfoil, there is a sudden drop in the drag force because of high pressure gradients interacting with the low pressure field induced by the vortex. As the vortex travels downstream along the length of the airfoil, separation at the surface as well as reverse flow occurs, which results in the decrease illustrated in Figure 6.12. For these reasons, there is a tremendous fluctuation in drag throughout the interaction followed by a divergence experienced by the flow impacting the wall of the cavity on the trailing edge segment of the airfoil. For Domain E however, the drag coefficient is higher than for the symmetric airfoil but after the interaction the values converge to the appropriate zero lift value of drag as per the drag shown for Domain A. 


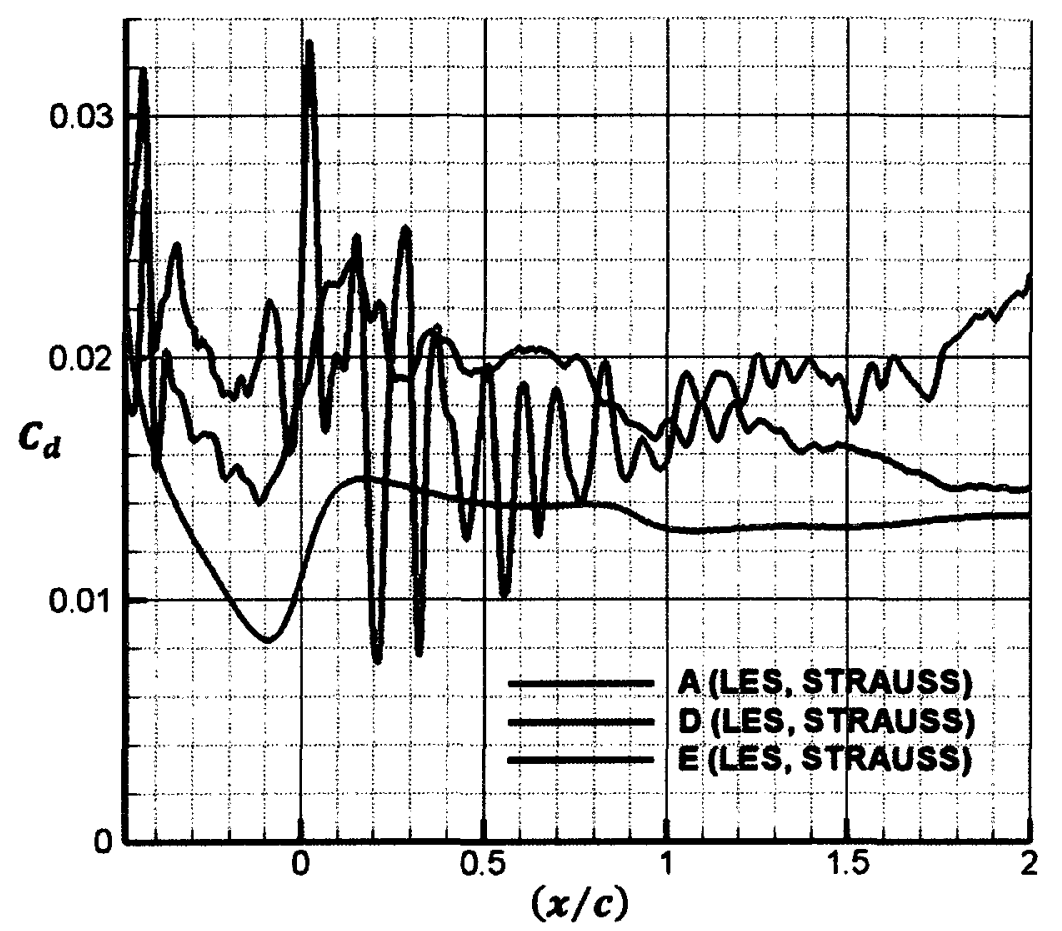

Figure 6.12: Drag Coefficient for Varying Vortex Location for Mitigation Study 1 (i.e. Domains A, D and $\mathbf{E})$

Finally, the moment coefficient for the same vortex locations as in Figure 6.11 and Figure 6.12 is presented in Figure 6.13. Illustrated in Figure 6.13 are the temporal variations of the moment coefficient for Domains A, D and E. The moment coefficient was calculated about the leading edge of the airfoil and is considered positive in the counter clockwise direction. Notice that the moment coefficient for each domain has an inverted trend of that of the lift coefficient. In Domain A, as the clockwise rotating vortex approaches the airfoil, there is a positive moment corresponding to a nose down pitching motion. Near the leading edge, the moment coefficient suddenly declines from its peak positive value to a negative value as the center of the vortex moves downstream. This is a result of the rotational sense of the tip vortex. The moment coefficient increases as the vortex approaches the airfoil due to the increase of the induced velocity and pressure 
fields around the airfoil. The maximum value of the moment coefficient coincides with the instance of the vortex center coinciding with the axis of the leading edge. Similar to the lift coefficient, the moment coefficient also converges to a zero value as the vortex slowly stops interacting with the airfoil. Domain D also has large fluctuations in the moment coefficient as per the lift and it is apparent that Domain E mitigates the pitching effect caused by the AVI when compared to Domain A.

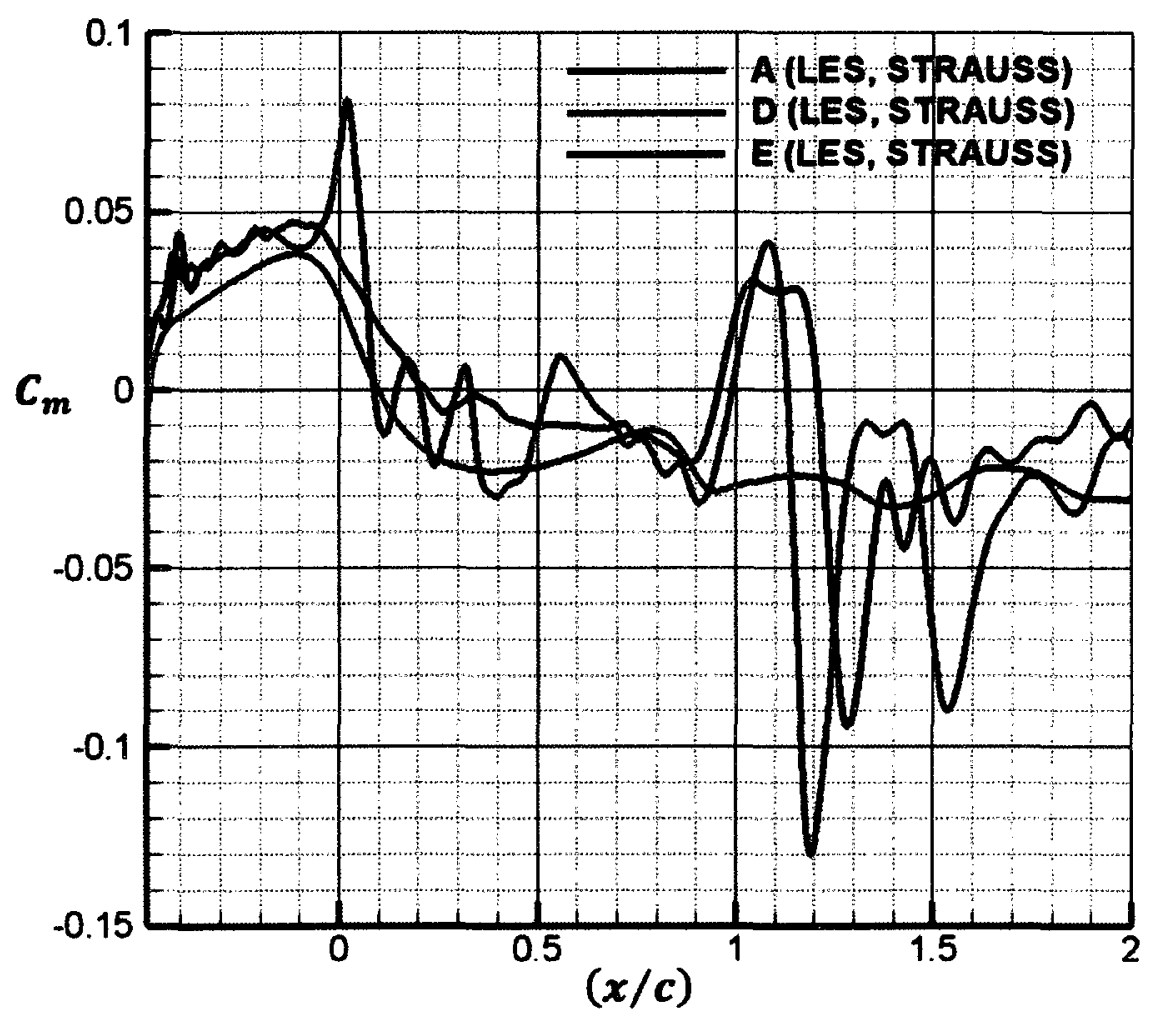

Figure 6.13: Moment Coefficient for Varying Vortex Location for Mitigation Study 1 (i.e. Domains $A, D$ and E)

As a result of the previous discussions, Domain $\mathrm{E}$ appears to provide benefits with respect to the variation in lift and moment with an acceptable penalty in drag during the interaction. However, Domain $\mathrm{D}$ appears to provide no benefits and shall not be examined for mitigation studies 2 and 3. To ensure that Domain $E$ mitigates the unsteady 
airloads during the worst case interaction, mitigation study 2 aims at investigating the case when the vortex has a miss distance of zero at the same velocity. These results shall now be presented and discussed.

\subsection{Mitigation Study 2}

Employing Domain A and Domain E based on the discussion above as well as the vortex parameters defined in Table 6.2, mitigation study 2 maybe performed. Note that the meshes for both Domain $\mathrm{A}$ and $\mathrm{E}$ as well as the vortex parameters and boundary conditions with the exception of the miss distance remain the same. Similar to mitigation study 1 , the second mitigation study shall begin with investigating the differences present in the velocity and pressure fields during the interaction with some discussion pertaining to the differences between the two miss distances in each study. The velocity and pressure fields are provided in Figure 6.14 and Figure 6.15 respectively. 

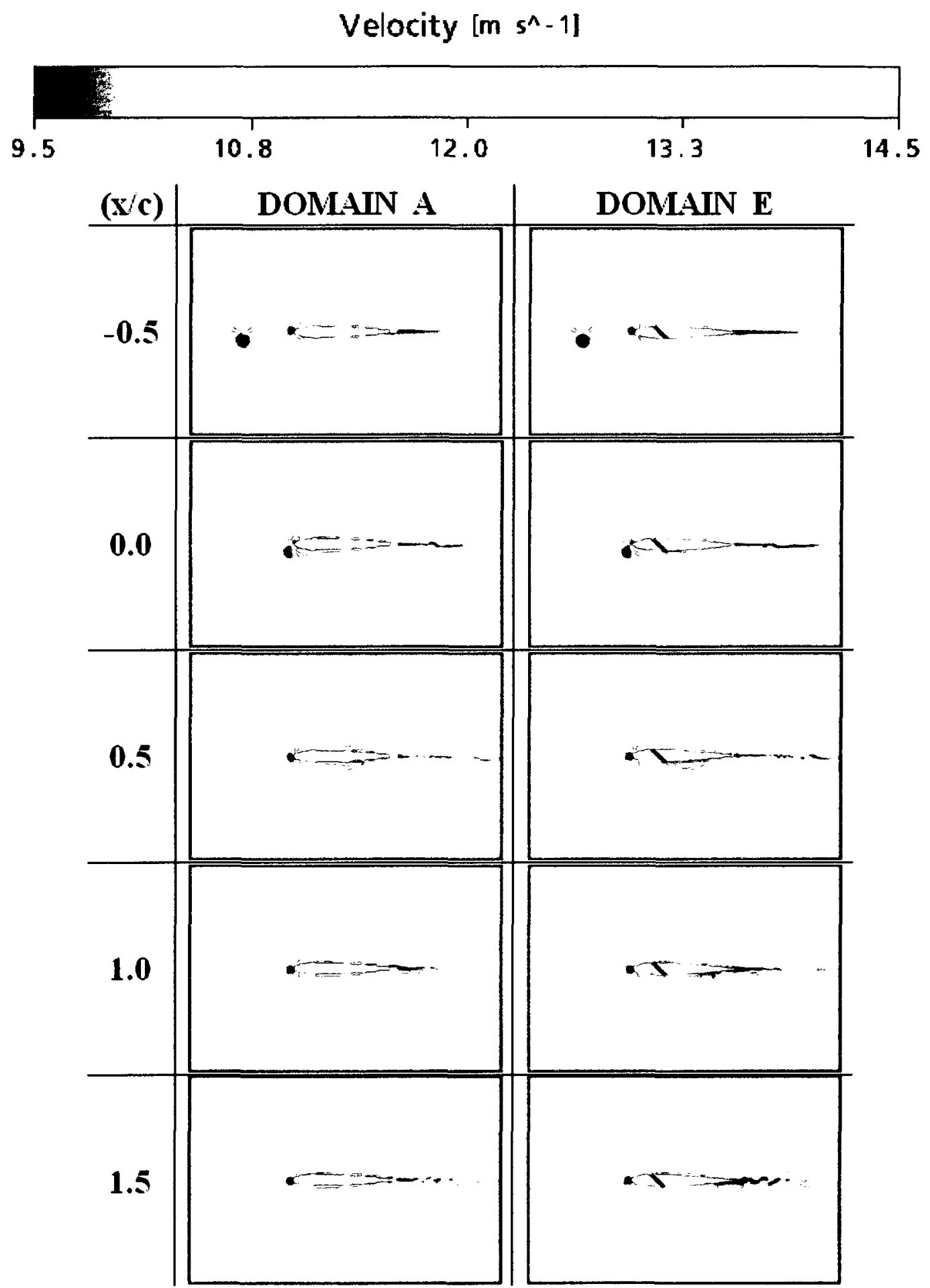

Figure 6.14: Velocity Fields for the Indicated Vortex Locations for Mitigation Study 2 

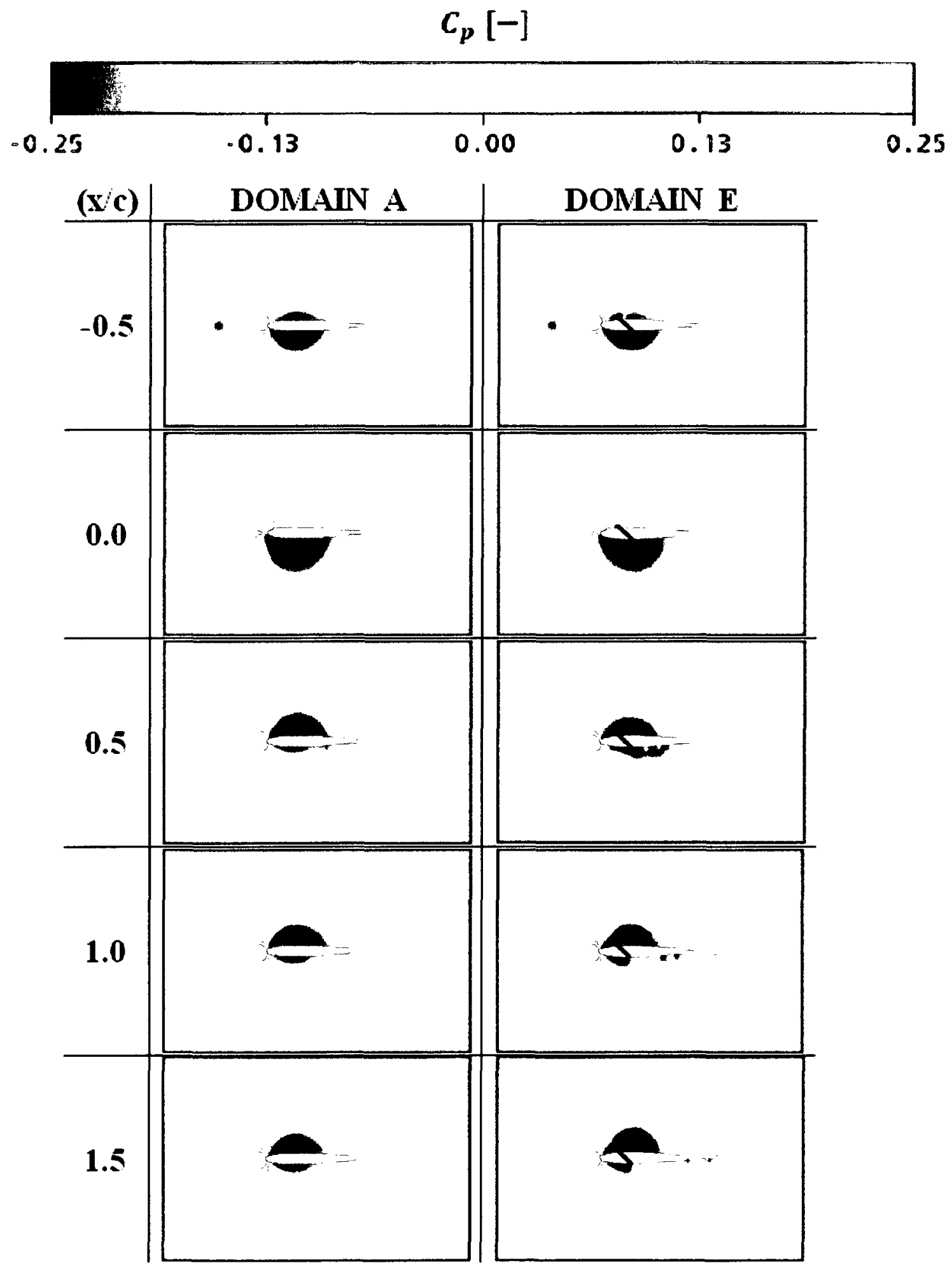

Figure 6.15: Pressure Fields for the Indicated Vortex Locations for Mitigation Study 2 
The time histories of the velocity field and the pressure field provide good insight regarding the flow physics of an airfoil-vortex interaction. As per mitigation study 1 , the airfoil-vortex interaction is described by three different phases. At the beginning of AVI, the vortex is upstream of the airfoil and moves towards the leading edge (i.e. $(x / c)=$ -0.5 to $(x / c) \approx 0$ ) as is illustrated in Figure 6.14 and Figure 6.15 for Domain A. Throughout this phase the vortex induces a downwash on the airfoil which causes the leading-edge stagnation point to move along the upper surface of the airfoil. This results in an increase in pressure over the airfoils upper surface. The second phase of AVI is identical to that described in Section 6.5, with the exception of the vortex being distorted in a much more significant manner. In the third phase, the vortex leaves the airfoil (i.e. $(x / c)>1)$ which generates wake perturbations. However, now the wake perturbations are much more significant than those found in mitigation study 1 . As the vortex convects towards the leading edge of the airfoil for Domain E, the same effects in the flow fields may be seen as per mitigation study 1 . As the vortex reaches the leading edge, the acceleration of the flow through the cavity is enhanced (as compared to mitigation study 1). This results in higher velocity on the lower surface of the trailing edge segment of the airfoil, which creates a region of lower pressure where acceleration results in a fluctuation of the airloads due to the variation of the pressure distribution over the airfoils surface. As the vortex approaches the mid chord of the blade regions of high velocity may be seen on each the upper and lower surfaces of the trailing edge segment of the airfoil. This induces an upwash on the lower surface and a counteracting downwash on the upper surface of the airfoil. The aforementioned shear layer is also present on each 
side of the airfoil which results in fluctuations of the drag force on the airfoil. A vortex is also formed from the lower surface of the cavity wall which causes fluctuations in both the lift and drag due to increased upwash velocities. As the vortex passes the trailing edge, the shed vortices from the cavity wall are present. However, the main tip vortex remains in a distorted state with lower velocities caused by the diffusion experienced during the interaction. In the final phase of the interaction the vortices (i.e. those shed and the tip vortex) perturb the wake in quite a significant manner which results in fluctuations in the blades airloads.

This study is also based on the aerodynamic coefficients (i.e. $C_{l}, C_{d}$ and $C_{m}$ ) and the $\mathrm{x}$-axis shall use the same definition as that provided in Section 6.5. The lift, drag and moment coefficient results for the aforementioned conditions shall now be provided.

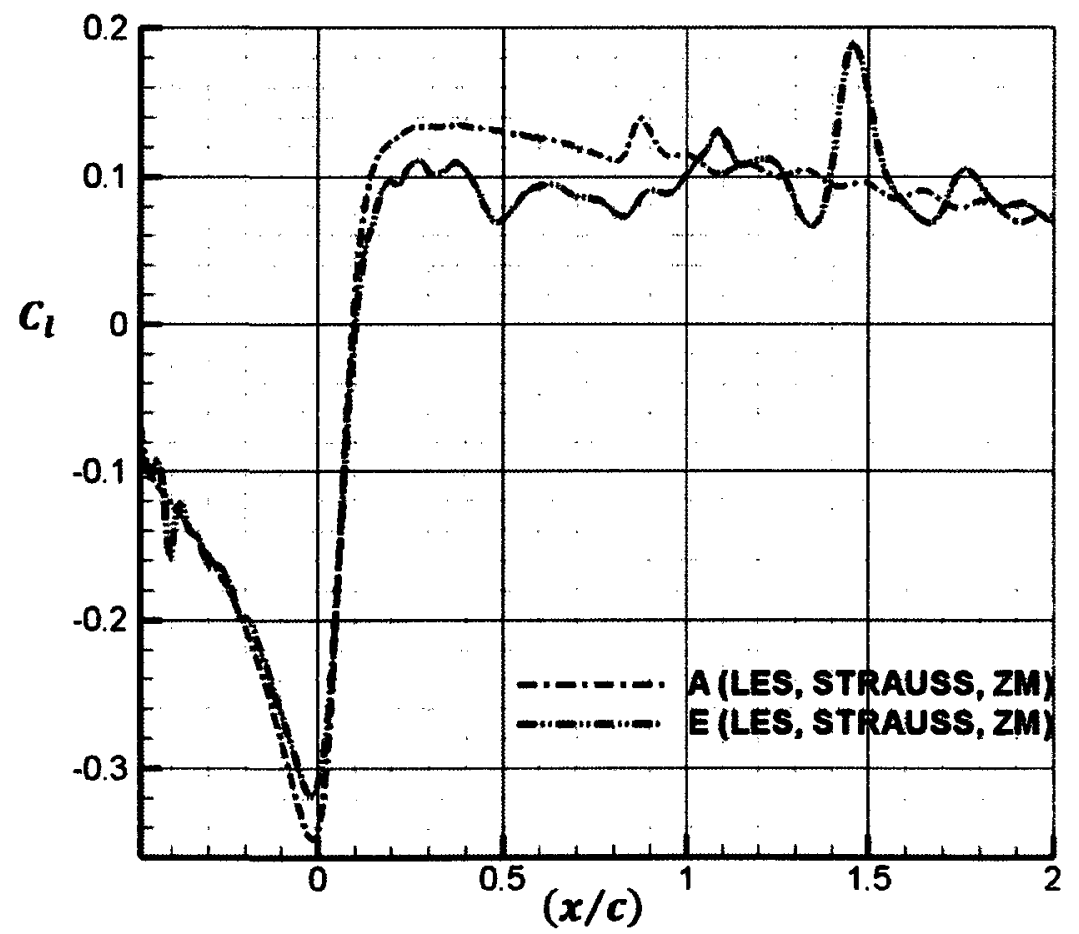

Figure 6.16: Lift Coefficient for Varying Vortex Location for Mitigation Study 2 (i.e. Domains $A$ and E) $(\mathrm{ZM} \rightarrow$ Zero Vertical Miss Distance) 


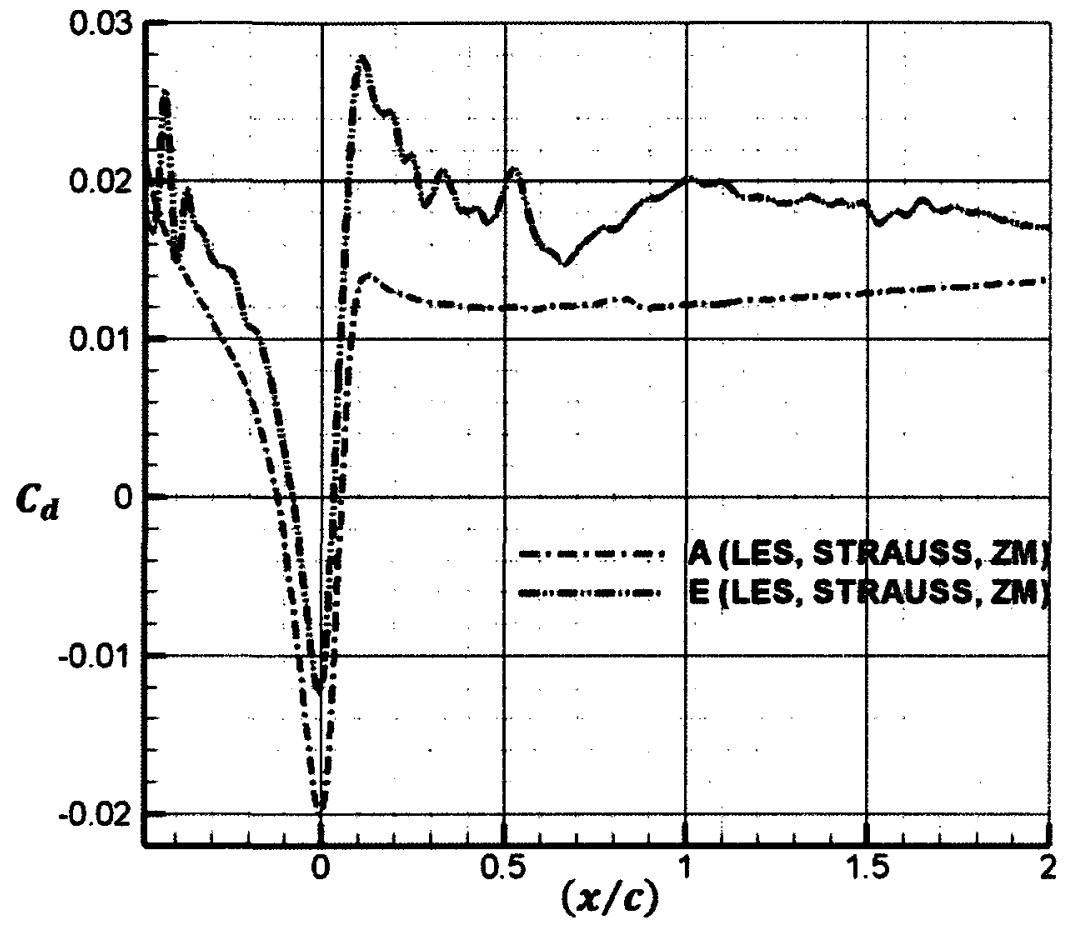

Figure 6.17: Drag Coefficient for Varying Vortex Location for Mitigation Study 2 (i.e. Domains A and E) (ZM $\rightarrow$ Zero Vertical Miss Distance)

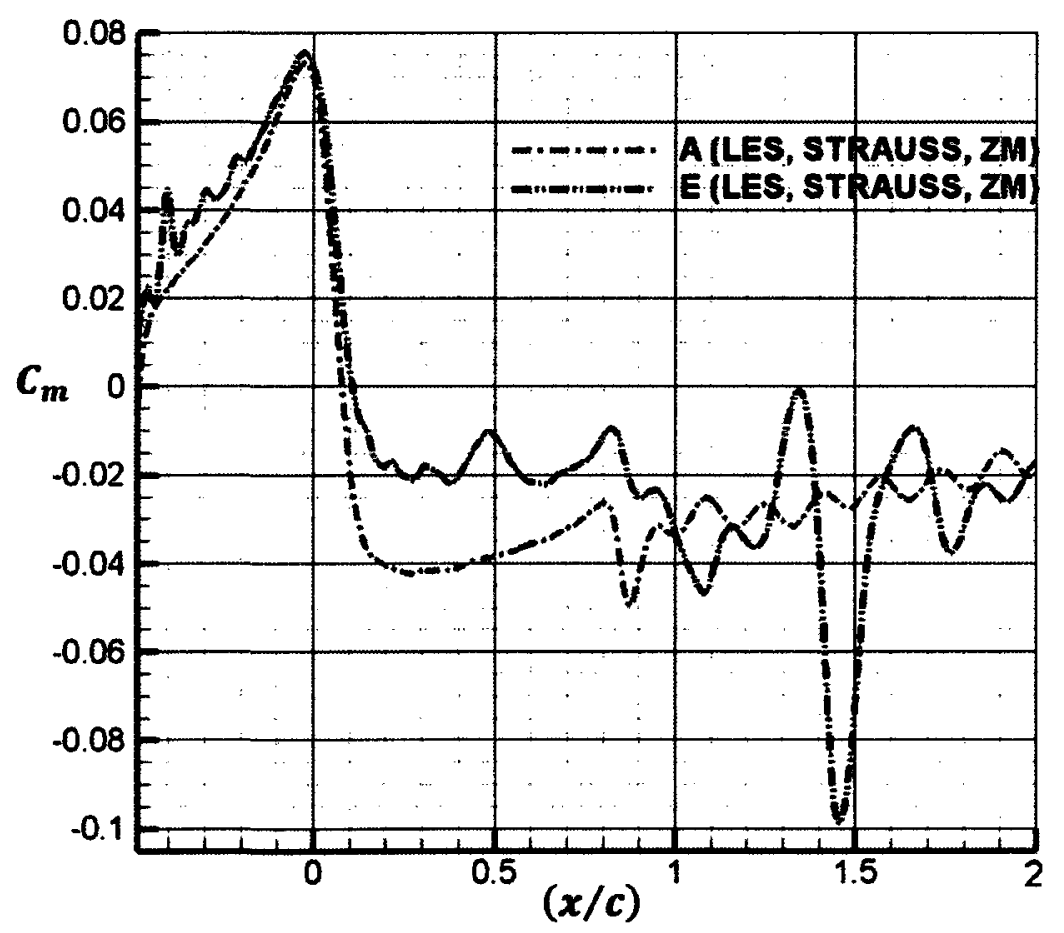

Figure 6.18: Moment Coefficient for Varying Vortex Location for Mitigation Study 2 (i.e. Domains A and $E)(Z M \rightarrow$ Zero Vertical Miss Distance) 
Figure 6.16 depicts the temporal variation of lift for Domains $\mathrm{A}$ and $\mathrm{E}$ for mitigation study 2. The same trends for Domain A discussed in Section 6.5 remain present with another feature worth mentioning. This feature is the fluctuations which are present in the third phase of the AVI due to the wake perturbations as the vortex interacts with the wake. Similarly, the phenomena discussed for Domain E in Section 6.5 also remain true. Notice that as the vortex approaches the leading edge of the airfoil, the cavity increases the minimum lift which is experienced, thereby further mitigating the interaction with respect to the variation of lift as well as the noise. For the benchmark airfoil, the incremental lift coefficient during the interaction (as defined in Section 6.4) was found to be 0.481 , whereas for the aforementioned flow conditions using the $45^{\circ}$ cavity, the incremental lift coefficient is given as 0.429 . The percent reduction of the incremental lift coefficient achieved through implementing the $45^{\circ}$ cavity when compared to the benchmark airfoil was found to be $5.24 \%$ for a freestream Mach number of 0.036 , a vortex strength of $0.9 \mathrm{~m}^{2} / \mathrm{s}$ and a zero vertical miss distance of the vortex. Based on Equation ( 6.3 ) and the discussion presented in Section 6.4, this leads to a $5.24 \%$ decrease in the sound pressure which is propagated to an observer.

Figure 6.17 represents the drag coefficient fluctuations present during the interaction for both Domains A and E. For Domains A and E, as the miss distance is reduced to the case of a fully parallel interaction, the aforementioned fluctuations in drag are amplified due to the stronger interaction present. The penalty in drag which results from the cavity remains, although the reverse flow experienced by the airfoil with the cavity is less than that of the symmetric airfoil. Note the higher peak in drag as the airfoil 
passes the leading edge of the airfoil with the cavity as compared to the symmetric airfoil as a result of the enhancement of the flow features discussed in Section 6.5.

Figure 6.18 illustrates that the peak moment for each domain is the same and occurs as the vortex reaches the leading edge. With the cavity, the effect of the pitching motion caused by the presence of the vortex is less for the airfoil than for the symmetric airfoil during the second phase of the interaction. In the final stage of the interaction, the additional vortices shed in Domain $\mathrm{E}$ result in large fluctuations in the moments produced during the interaction as they pass the trailing edge of the airfoil.

As a result of the previous discussions, Domain E appears to provide further benefits for variation in lift and moment with an acceptable penalty in drag during the interaction. This remains true until the vortex reaches the trailing edge of the airfoil where the fluctuations in lift and moment are further excited. Mitigation study 3 aims to investigate whether the benefits shown by Domain $\mathrm{E}$ remain while the freestream velocity is increased and the miss distance is maintained as zero. These results shall now be presented and discussed in full.

\subsection{Mitigation Study 3}

As per mitigation study 2, Domain A and Domain E are also used for mitigation study 3. The vortex parameters for this study are defined in Table 6.2 and the boundary conditions are provided in Table 6.3 and Table 6.4. Mitigation study three also employs a zero miss distance (as per the second study). However the freestream velocity has been increased to Mach 0.25 . This study has been performed to demonstrate the influence of the freestream Mach number (and Reynolds number) on the airloads created during a 
parallel BVI as well as the hole's effect in a situation with a higher freestream velocity. This study is also based on the aerodynamic coefficients (i.e. $C_{l}, C_{d}$ and $C_{m}$ ) and the xaxis shall use the same definition as that provided in Section 6.5.

The lift, drag and moment coefficient results for the aforementioned conditions are shown in Figure 6.19 through Figure 6.21.

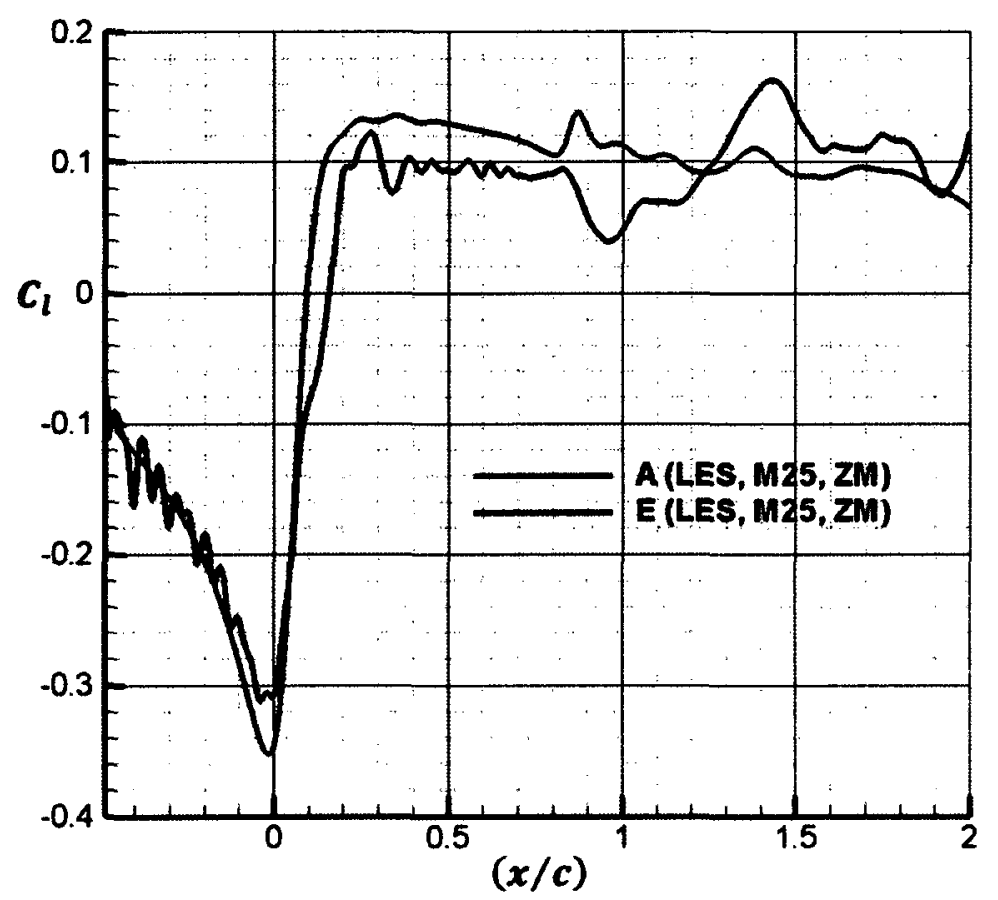

Figure 6.19: Lift Coefficient for Varying Vortex Location for Mitigation Study 3 (i.e. Domains A and E) $(\mathbf{Z M} \rightarrow$ Zero Vertical Miss Distance) 


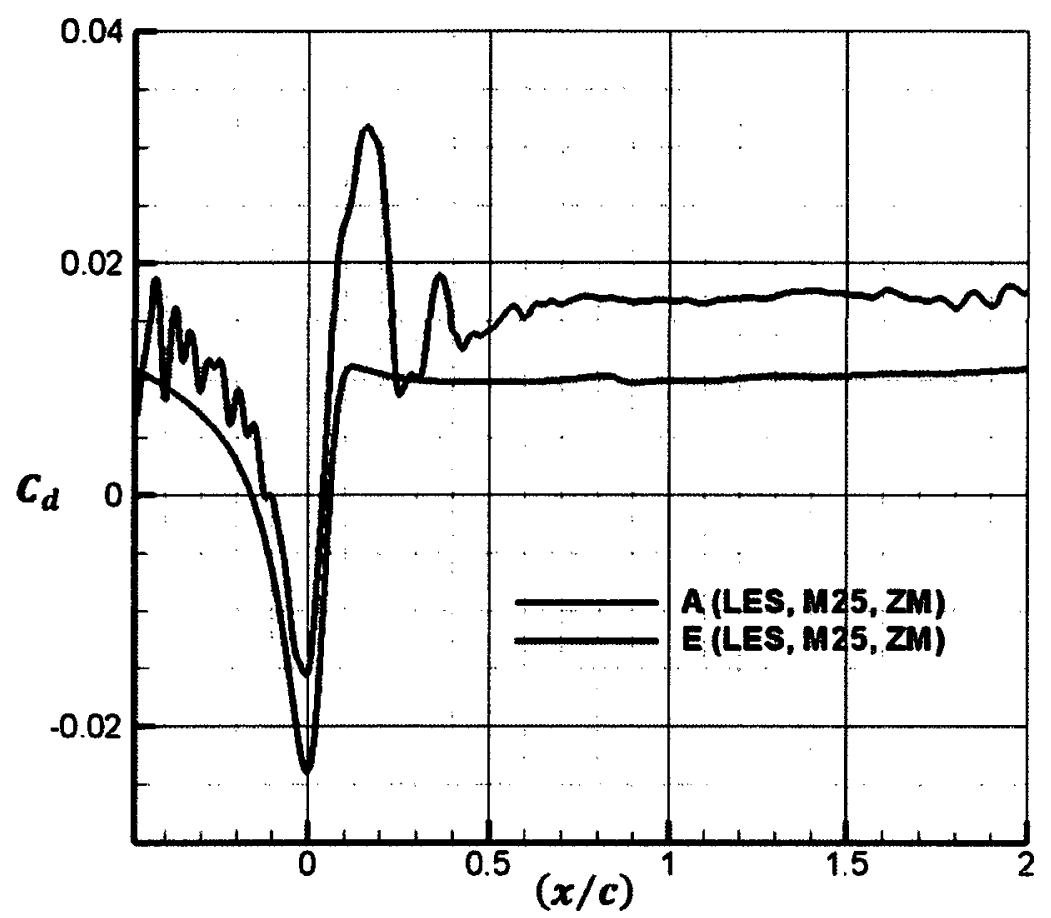

Figure 6.20: Drag Coefficient for Varying Vortex Location for Mitigation Study 3 (i.e. Domains A and E) $(\mathbf{Z M} \rightarrow$ Zero Vertical Miss Distance)

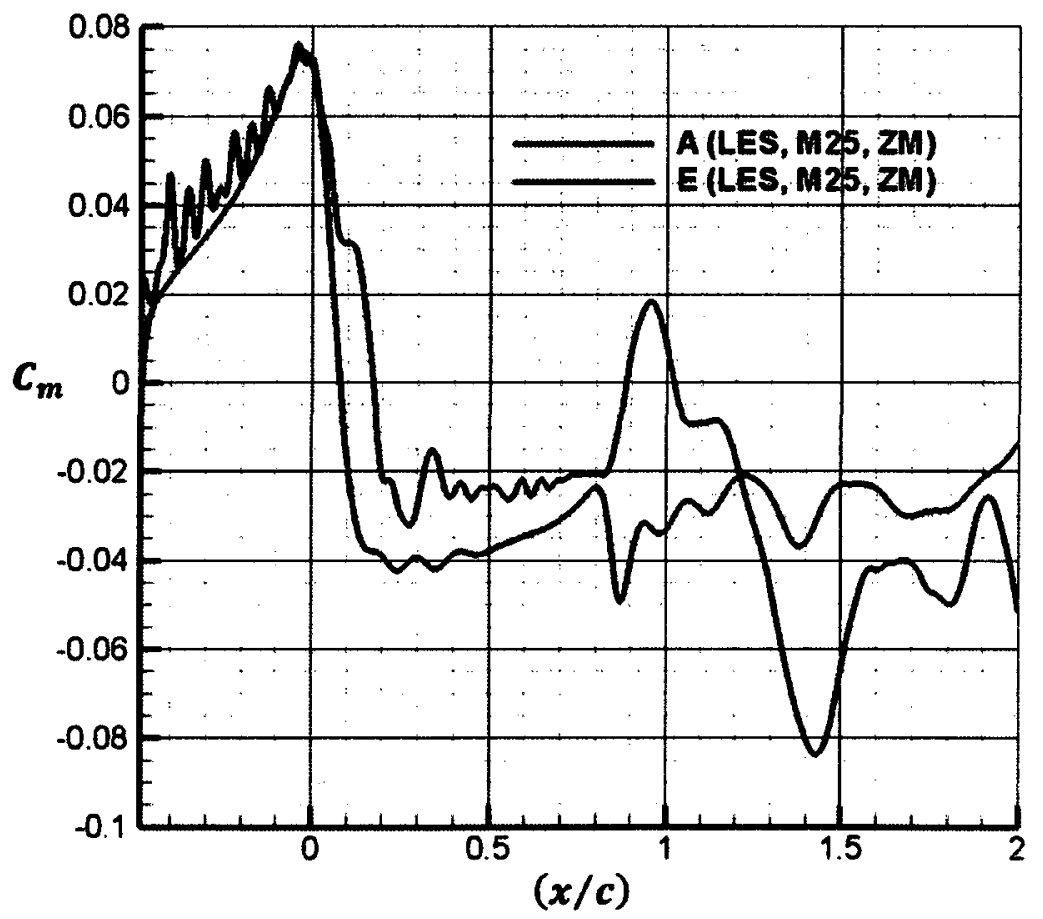

Figure 6.21: Moment Coefficient for Varying Vortex Location for Mitigation Study 3 (i.e. Domains A and E) (ZM $\rightarrow$ Zero Vertical Miss Distance) 
Figure 6.19 depicts the temporal variation of lift for Domains $\mathrm{A}$ and $\mathrm{E}$ for mitigation study 3. The trends discussed in Section 6.6 for Domain A remain identical for mitigation study 3. For Domain E, it becomes apparent that as the vortex approaches the airfoil with a higher freestream velocity, the lift acting on the airfoil fluctuates much more rapidly while its mean value follows the same trend observed for mitigation study 2. This may be attributed to the increased strength of the vortex employed in the domain, which thus results in higher fluctuations. For the benchmark airfoil, the incremental lift coefficient during the interaction (as defined in Section 6.4) was found to be 0.488 , whereas for the aforementioned flow conditions using the $45^{\circ}$ cavity, the incremental lift coefficient is given as $\mathbf{0 . 4 3 5}$. The percent reduction of the incremental lift coefficient achieved through implementing the $45^{\circ}$ cavity when compared to the benchmark airfoil was found to be $5.31 \%$ for a freestream Mach number of 0.25 , a vortex strength of 6.3 $\mathrm{m}^{2} / \mathrm{s}$ and a zero vertical miss distance of the vortex. Based on Equation ( 6.3 ) and the discussion presented in Section 6.4, this leads to a $5.31 \%$ decrease in the sound pressure which is propagated to an observer. Furthermore, it is apparent that the spike in lift after the vortex leaves the trailing edge is less impulsive as the freestream velocity is increased (i.e. occurs over a larger vortex distance). It also occurs at an earlier point as the freestream velocity is increased. The variation in lift remains less significant for the airfoil with the cavity than the symmetric airfoil as the freestream velocity is increased. Figure 6.20 illustrates the variation in drag for the increased freestream velocity case. The trends for Domain A remain the same as per the lower freestream velocity investigation. 
Domain E illustrates the fluctuations mentioned in Section 6.6 although the higher freestream velocity has the effect of washing out the fluctuations caused by the vortex interacting with the airfoil. Note the penalty in drag remains for the airfoil with the cavity. Like the effects mentioned in Section 6.6 regarding the moment variation during the interaction, Domain $\mathrm{E}$ mitigates the effect of the fluctuating moments which act on the airfoil. However, the larger fluctuations which result from the multiple vortices interacting with the airfoil are delayed and less substantial as the freestream velocity is increased as can be seen in Figure 6.21.

Based on the above discussions, it is evident that the airfoil with a $45^{\circ}$ cavity placed at the quarter chord location effectively mitigates the unsteady lift and moments while increasing the drag on the airfoil compared to the symmetric airfoil. This mitigation has been achieved since the incremental lift coefficient (linked to the noise propagated to an observer) has been decreased as the vortex passes the leading edge of the airfoil with the $45^{\circ}$ cavity. This seemingly holds true for all the flight conditions investigated (i.e. powered descent and higher forward flight speeds). In an attempt to further mitigate the interaction experienced as the vortex convects downstream past the trailing edge several systems may potentially decrease the fluctuations in this phase of the interaction. One system which may be employed involves adding an additional $45^{\circ}$ cavity in the same location, but in the opposite direction. Also, a valve should be implemented to determine which cavity is open. Throughout the interaction the cavity presented above should be employed. However, as the vortex reaches its final phase of the interaction, the $-45^{\circ}$ cavity should be closed and the $+45^{\circ}$ cavity should be opened in an attempt to alleviate 
the fluctuations in lift and moment in this phase. An alternate method would be to investigate a series of $-45^{\circ}$ cavities placed along the airfoils surface. Perhaps a larger cavity should be employed or an alternate angle, however the size and angle of the cavity will be dependent on the flight condition experienced by the rotorcraft. 


\section{Chapter 7: Conclusions}

The present analysis focused on verification and validation of using LES as a means of predicting the aerodynamics involved in an airfoil-vortex interaction. This investigation also focused on the mitigation of AVI through the use of passages through the airfoil. In this analysis, the vortex created with the Lamb-Oseen model was released upstream of the airfoil and the unsteady flow field results were recorded at regular time intervals. These time intervals correspond to different locations of the vortex core along the trajectory of the vortex. They were used to determine the aerodynamic coefficients. In the validation and verification analysis, both grid and domain convergence were analyzed. Through the grid convergence study, it was determined that a domain containing 760,464 nodes in a structured grid was sufficient for resolving the lift and moment coefficients; however, a deviation was noted for the drag coefficient. Similarly, the domain convergence analysis further iterated that the domain was acceptable. Thus, for the verification and validation analysis a domain with a thickness of $12 \%$ of the chord was employed in order to minimize the computational time which was required. By employing the convergent domain and grid, both LES and SST based URANS simulations were performed to investigate the differences in the results obtained through the aforementioned CFD methods. For each simulation, it was ensured that each had 
sufficient temporal resolution through employing a Courant number less than unity (i.e. the same domain and time steps were used). Based on the verification analysis, it was concluded that if the Courant number is maintained below unity, the vortex does not experience numerical dissipation as it convected past the NACA 0012 airfoil. Upon verifying the computational method, a validation analysis was performed with the data presented by Straus et al. (1990) and Renzoni (1987). The experimental data obtained by Straus et al. (1990) was found to be within 1\% of the data obtained from LES until the vortex had just passed the leading edge of the airfoil. At this point, the data begins to diverge for several conjectured reasons. The inviscid data obtained by Renzoni (1987) was found to agree with the LES data until the mid chord of the airfoil, at this point the differences attributed to viscosity may be seen.

The second portion of the investigation was concerned with mitigating the effects of the vortex on the unsteady aerodynamic loads experienced by the blades. In an attempt to investigate the geometries effect on the overall interaction, NACA 0012 airfoils were outfitted with two different cavities. The first cavity was a straight vertical hole centered at the quarter chord location with a width of $5 \%$ of the chord. The second cavity which was investigated employed a cavity at $-45^{\circ}$ from the vertical also at centered about the quarter chord location with a width of $5 \%$ of the chord. The NACA 0012 airfoil without a cavity was employed as a benchmark case. A preliminary study that employed the conditions used throughout the validation analysis was performed to determine which cavity provided the most benefit (if any) with respect to the unsteady aerodynamic loads acting on the blade during the interaction. By comparing the aerodynamic coefficients 
obtained for each cavity with the benchmark airfoil, it was established that the $-45^{\circ}$ cavity mitigated the interaction (reduction of the incremental lift coefficient was 3.3\%) throughout most of the interaction however as the vortex left the airfoil it increased the fluctuating airloads. The straight cavity however provided no benefits whatsoever to the fluctuations experienced by the blade loading. Using the slanted cavity and the symmetric airfoil two further studies were performed to ensure that the slanted cavity mitigated the interaction for a variety of conditions. The first study involved decreasing the miss distance between the vortex and blade axes to zero, in which the incremental lift coefficient (and the sound pressure propagated to an observer) was reduce $5.2 \%$ through implementation of the $45^{\circ}$ cavity (relative to the benchmark airfoil). While the second study maintained the zero miss distance and increased the freestream velocity to a Mach number of 0.25 , which was found to mitigate the interaction by $5.3 \%$ relative to the benchmark airfoil if a $45^{\circ}$ cavity is employed. Throughout these parametric studies, it was determined that the mitigation occurs regardless of the conditions imposed on vortex and flow.

Finally, although it is relatively expensive from a computational standpoint, LES may be used to simulate the fluid structures present during a blade-vortex interaction. This cannot be captured with sufficient accuracy using more traditional methods. It is hence a suitable and affordable tool which may be used in the prediction of the aerodynamics involved throughout a blade-vortex interaction event. 


\section{References}

Althoff, S.L., Elliott, J.W., and Sailey, R.H., 1988. "Inflow Measurements Made with a Laser Velocimeter on a Helicopter Model in Forward Flight," NASA Technical Memorandum 100544.

Ananthan, S., and Leishman, J.G., 2004. "Rotor wake Dynamics During Combat Maneuvers," $60^{\text {th }}$ Forum of the American Helicopter Soc. International, Baltimore, MD. June 7-12.

Anderson, J.D., 2007. Fundamentals of Aerodynamics, $4^{\text {th }}$ edition, New York, McGraw Hill.

Baeder, J.D., 1997. "Passive Design for Ísolated Blade-Vortex Interaction Noise," $53^{\text {rd }}$ Annual Forum of the American Helicopter Soc., Virginia Beach, VA, April 29 - May 1.

Bagai, A. and Leishman, J.G., 1992. "A Study of Rotor Wake Developments and Wake/Body Interactions in Hover using Wide-Field Shadowgraphy," J. of the American Helicopter Soc., 37 (4), pp. 48-57.

Batchelor, G.K., 1964. "Axial flow in trailing line vortices," J. Fluid Mech., 20, pp. 645-658.

Bausch, W.E., Munch, C.L., and Schlegel, R.G., 1971. "An Experimental Study of Helicopter Rotor Impulsive Noise," USAAVLABS TR 70-72, June.

Bellamy-Knights, P.G., 1970. “An unsteady two-cell vortex solution of Navier-Stokes equations,” J. Fluid Mech., 41, pp. 673-687.

Berenger, T., Favier, D., Maresca, C. and Berton, E., 1997. "Experimental and numerical investigation of rotor aerodynamics in forward flight," Journal of Aircraft, 34 (3), pp. 394-399.

Bhagwhat, M.J. and Leishman, J.G., 2000. "Correlation of Helicopter Tip Vortex Measurements," AlAA J., 38 (2), pp. $301-308$.

Bhagwhat, M.J. and Leishman, J.G., 2002, "Generalized Viscous Vortex Core Models for Application to FreeVortex Wake and Aeroacoustic Calculations," $58^{\text {th }}$ Annual Forum of the American Helicopter Soc. International, Montreal, Canada, June 11-13.

Biggers, J.C. and Orloff, K.L., 1975. "Laser Velocimeter Measurements of the Helicopter Rotor-Induced Flow Field," J. of the American Helicopter Soc., 20 (1), pp. 2-10.

Biggers, J.C., Orloff, K.L., and Lemmer, O.J., 1977. “Measurements of Helicopter Rotor Tip Vortices," $33^{\text {rd }}$ Annual National Forum of the American Helicopter Soc., Washington DC, May 9-11.

Biggers, J.C., Orloff, K.L., and Lemmer, O.J., 1977. "Laser Velocimeter Measurements of Two-Bladed 
Helicopter Rotor Flow Fields," NASA TM X-73238.

Booth, E.R. and Yu, J.C., 1986. "Two-dimensional blade-vortex flow visualization investigation," AIAA Journal, 24 (9), pp. 1468-1473.

Booth, E.R. and Yu, J.C., 1990. "Experimental observations of two-dimensional blade-vortex interaction," AlAA Journal, 28 (8), pp. 1353-1359.

Bossel, H.H., 1968. "Stagnation Criterion for Vortex Flows," AIAA Journal, 6 (6), pp. 1 192-1193.

Bossel, H.H., 1969. "Vortex Breakdown Flowfield," Physics of Fluids, 12 (3), pp. 598-508.

Bossel, H.H., 1970. "Use of exponentials in the Integral Solution of the Parabolic Equations of Boundary Layer, Wake, Jet and Vortex Flows," Journal of Computational Physics, 5, pp. 359-382.

Bossel, H.H., 1971. "Vortex Computation by the Method of Weighted Residuals Using Exponentials," AIAA Journal, 9 (10), pp. 2027-2034.

Bossel, H.H., 1973. "Swirling Flows in Streamtubes of Variable Cross Section," AIAA Journal, 11 (8), pp. 1161 1165.

Boxwell, D.A, Schmitz, F.H., and Hanks, M.L., 1975. "In-Flight Far-Field Measurements of Helicopter Impulsive Noise," European Rotorcraft and Powered Lift Aircraft Forum, Southampton, England, September.

Bradshaw, P., 1983. "The Analogy Between Streamline Curvature and Buoyancy in Turbulent Shear Flows," J. of Fluid Mechanics, 33, pp. 177-191.

Brentner, K.S., 1997, "A Superior Kirchhoff Method for Aeroacoustic Noise Prediction: The Ffowcs-Williams Hawkings Equation," 134" Meeting of the Acoustical Soc. Of America, San Diego, CA, Dec. 1-5.

Brentner, K.S., 1997, "Helicopter Rotor Noise Prediction: Background, Current Status, and Future Directions," University of Tennessee Space Institute Seminar, Chattanooga, TN, December.

Brentner, K.S., and Farassat, F., 2003. "Modeling Aerodynamically Generated Sound of Helicopter Rotors," Progress in Aerospace Sciences, 39 (2-3), pp. 83-120.

Brooks, T.F., Booth, E.R., Boyd, D.D., Splettstoesser, W.R., Schultz, K.J., Roland, N., Georg, H., and Stregy, O., 1991. "HHC study in the DNW to Reduce BVI Noise-An Analysis," International Technical Specialists Meeting on Rotor Acoustics and Rotor Fluid Dynamics, AHS/RAeS, Valley Forge, Pa, Oct. 15-17.

Burgers, J.M., 1940. "Application of a model system to illustrate some points of the statistical theory of free turbulence." Proc. in Academic Science, Amsterdam, Netherlands, 1 (1), pp. 2-12.

Burgers, J.M., 1948. "A mathematical model illustrating the theory of turbulence," Adv. Appl. Mech., 1, pp. 197199.

Burwash, W.M., 2007. "Particle Image Velocimetry Measurements of an Airfoil-Vortex Interaction Event in a Two-Dimensional Wind Tunnel," M.A.Sc. Thesis, Carleton University.

Caradonna, F.X. and Strawn, R.C., 1988. "An experimental and computational study of rotor-vortex interactions," Vertica, 12 (4), pp. 315-327. 
Caradonna, F.X., and Tung, C., 1981. "Experimental and Analytical Studies of a Model Helicopter Rotor in Hover," $7^{\text {th }}$ European Rotorcraft Forum, Garmisch-Partenkirchen, Germany, Sept. 22-25.

Caradonna, F.X., Kitaplioglu, C., McCluer, Baeder, J.D., Leishman, J.G., Berezin, C., Bridgeman, J., Burley, C.R., Eipstein, R., Lyrintzis, A., Koutsavdis, E., Rahier, G., Jobard, J., and J. Rule, J., 1997. “A Review of Methods for the Prediction of BVI Noise," American Helicopter Soc. Technical Specialists Meeting in Rotorcraft Acoustics and Aerodynamics, Williamsburg, VA, Oct. 28-30.

Cesnik, C. and Shin, S., 2001. "On the modeling of integrally actuated helicopter blades," Int.J.of Solids and Struct.,38, pp. 1765-1789.

Cesnik, C. and Shin, S., 2001. "On the Twist Performance of a multiple-cell active helicopter blade," J.of Smart Materials and Structures, 10, pp. 53-61.

Charles, B., Hassan, A.A., Sankar, L.N., and Tadghighi, H., 1994. "Effects of Leading and Trailing Edge Flaps on the Aerodynamics of Airfoil/Vortex Interaction," J. of the American Helicopter Soc., 39 (2), pp. 35-46.

Charles, B.D., 1975. "Acoustic Effects of Rotor-Wake Interaction During Low-Power Descent," American Helicopter Society Symposium on Helicopter Aerodynamic Efficiency, Hartford, CT, March.

Chigier, N.A., and Corsiglia, V.R., 1971. "Tip Vortices - Velocity Distributions," $27^{\text {th }}$ Annual Forum of the American Helicopter Soc., Washington DC, May.

Cook, C.V., 1972. "The Structure of the Rotor Blade Tip Vortex," In: AGARD CP-111.

Cotel, A.J. and Breidenthal, R.E., 1999. "Turbulence Inside a Vortex," Physics of Fluids, 11 (10) pp. 3026-3029.

Cotel, A.J., 2002. “Turbulence Inside a Vortex: Take Two," Physics of Fluids, 14 (8) pp. 2933-2934.

Cox, C.R., 1963. "Helicopter Noise and Passive Defense," American Helicopter Soc. Forum.

Cox, C.R., 1977. "Helicopter Rotor Aerodynamic and Aeroacoustic Environments," AIAA Paper 77-1338, October.

Cox, C.R., and Lynn, R.R., 1962. "A Study of the Origin and Means of Reducing Helicopter Noise," TCREC TR 62-73, November.

Coyne, A. J., Bhagwat, M. J. and Leishman, J.G., 1997. "Investigation into the Rollup and Diffusion of Rotor Tip Vortices using Laser Doppler Velocimetry," Proceedings of the $53^{\text {rd }}$ AHS Annual Forum, Virginia Beach, VA, Apr. 29-May 1.

Dacles-Mariani, J., Kwak, D. and Zilliac, G., 1999. "On Numerical Errors and Turbulence Modeling in Tip Vortex Flow Prediction," Int. J. Numer. Meth. Fluids, 30, pp. 65-82.

Davidson, L., 2003. “An Introduction to Turbulence Models," Goteberg, Chalmers University of Technology.

Dawson, S., 1995. "Wind Tunnel Test of an Active Flap Rotor: BVI Noise and Vibration Reduction," $51^{\text {st }}$ Annual Forum of the American Helicopter Soc., Fort Worth, TX, May 9-11.

Devenport, W. J., Rife, M. C., Liapis, S. I., and Follin, G. J. 1996. "The structure and development of a wing-tip vortex," Journal of Fluid Mechanics, 312, pp. 67-106. 
Donaldson, C.P. and Sullivan, R.D., 1960. "Behaviour of solutions of the Navier-Stokes equations for a complete class of three-dimensional viscous vortices," Proc. of the heat transfer fluid mechanics conf., Stanford, pp. 16-30.

Doolan, C.J., Coton, F.N. and Galbraith, R.A.M., 1999. "Measurement of three-dimensional vortices using,a hot wire anemometer," in 30th AIAA Fluid Dynamics Conference, Norfolk, VA, June.

Dosanjh, D.S., Gasparek, E.P., and Eskinazi, S., 1962. "Decay of Viscous Trailing Vortex," Aeronautical Quarterly, 13, pp. 167-188.

Edwards, B.D. and Cox, C., 2002. "Revolutionary Concepts for Helicopter Noise Reduction-S.I.L.E.N.T. Program," NASA/CR-2002-211650, May.

Egolf, T.A. and Landgrebe, A.J., 1983. "Helicopter Rotor Wake Geometry and Its Influence in Forward Flight Volume I - Generalized Wake Effects on Rotor Airloads and Performance," NASA CR-3726, October.

Elliott, J.W., Althoff, S.L., and Sailey, R.H., 1988. "Inflow Measurements Made with a Laser Velocimeter on a Helicopter Model in Forward Flight," Vols. II and III, Rectangular Blades-Advance Ratios of 0.23 and 0.30, NASA TM-100542, TM-100543.

Farrasat, F., 1975. "Theory of Noise Generation from Moving Bodies with an Application to Helicopter Rotors," NASA TR R-451, December.

Fonseca, G.F., Bodstein, G.C.R. and Hirata, M.H., 2003. "Numerical Simulation of Inviscid Incompressible TwoDimensional Airfoil-Vortex Interaction in Ground Effect," Journal of Aircraft, 40 (4), pp. 653-661.

George, A.R., 1978. "Helicopter Noise: State-of-the-Art," J. of Aircraft, 15 (11), pp. 707-715.

Germano, M., Piomelli, U., Moin, P. and Cabot, W.H., 1991. "A Dynamic Subgrid-Scale Eddy Viscosity Model," Physics of Fluids A (Fluid Dynamics), 3 (7), pp. 1760-1765.

Glabel, H., Kloppel, V. and Rudolph, S., 2001. "Neural control of helicopter blade-vortex interaction noise," in SPIE 8th International Symposium on Smart Structures and Materials, Newport Beach, CA, March.

Gowanlock, D. K. and Matthewson, C. S., 1999. "Control of Rotor Tip Vortices," The $37^{\text {th }}$ AIAA Aerospace Sciences Meeting and Exhibit, Reno, NV, Jan 11-14.

Grant, I. and Parkin, P., 2000. "A DPIV study of the trailing vortex elements from the blades," Experiments in Fluids, 28, pp. 368-376.

Grant, I., Pan, X., Wang, X. and Stewart, N., 1994. "Correction for viewing angle applied to PIV data obtained in aerodynamic blade vortex interaction studies," Experiments in Fluids, 18, pp. 95-99.

Green, M., Parker, K., and Soria, J., 2005. "2D DPIV of a pitching aerofoil," in 4th Australian Conference on Laser Diagnostics in Fluid Mechanics and Combustion, pp. 53-56.

Green, R.B., Doolan, C.J. and Cannon, R.M., 2000. "Measurments of the orthogonal blade vortex interaction using a particle image velocimetry technique," Experiments in Fluids, 29, pp. 369-379.

Han, Y. O. and Leishman, J. G., 2003. "Investigation of Helicopter Rotor-Blade-Tip-Vortex Alleviation Using a Slotted Tip," Proceedings of the $59^{\text {th }}$ AHS, Annual Forum, Phoenix, AZ, May 7-10.

Han, Y. O. and Leishman, J. G., 2004. "Investigation of Helicopter Rotor-Blade-Tip-Vortex Alleviation Using a 
Slotted Tip," AIAA Journal, 42.(3), March.

Han, Y., Leishman, J.G., and Coyne, A., 1997. "Measurements of the Velocity and turbulence Structure of a Rotor Tip Vortex," AIAA J., 35 (3), pp. 477-485.

Hardin, J. C. and Lamkin, S. L., 1997. "Concepts for Reduction of Blade/Vortex Interaction Noise," J.Aircraft, 24 (2).

Hoad, D. R., 1980. "Helicopter Model Scale Results of Blade-Vortex Interaction Impulsive Noise as Affected by Tip Modification," Proceedings of the $36^{\text {th }}$ Annual American Helicopter Society Forum, Washington, DC, May 13-15.

Hoad, D.R., 1983. "Preliminary Rotor Wake Measurements with a Laser Velocimeter," NASA TM 83246.

Hoffman, E.R. and Joubert, P.N., 1963. "Turbulent Line Vortices," J. of Fluid Mechanics, 16, pp. 395-411.

Holzapfel, A., Hofbauer, T., Gerz, T., and Schumann, U., 2001. "Aircraft Wake Vortex Evolution and Decay in Idealized and Real Environments: Methodologies, Benefits and Limitations," Euromech Colloquium.

Hooper, W.E., 1983. "The Vibratory Airloading of Helicopter Rotors," 9 th European Rotorcraft Forum, Stresa, Italy, Sept. 13-15.

Hopfinger, E.J. and van Heijst, G.J.F., 1993. "Vortices in rotating fluids," Annual Revue Fluid Mech., 25, pp. 241-289.

Horner, M.B., Galbraith, R.A.M., Coton, F.N., Stewart, J.N. and Grant, I., 1996. "Examination of vortex deformation during blade-vortex interaction," AIAA Journal, 34 (6), pp. 1188-1194.

Horner, M.H., Steward, J.N., Galbraith, R.A.McD., Grant, I., and Coton, F.N., 1994. "An Examination of Vortex Deformation During Blade Vortex Interaction Using Particle Image Velocimetry," $19^{\text {th }}$ ICAS Congress, Anaheim, CA, Sept.

Hubbard, J.E.,Jr. and Leighton, K.P., 1983. "A Comparison of Model Helicopter Rotor Primary and Secondary Blade Vortex Interaction Blade Slap," AIAA Paper 83-0723, April.

Ilie, M., 2008. "Aerodynamic, Aeroacoustic, and Aeroelastic Investigations of Airfoil-Vortex Interaction using Large-Eddy Simulation," PhD thesis, Carleton University.

Ilie, M., Nitzsche, F., and Matida, E., 2008. "Influence of angle of attack on the helicopter blade-vortex interaction mechanism of interaction using Large Eddy Simulation," $46^{\text {th }}$ AIAA Aerospace Sciences Meeting and Exhibit, Reno, Nevada, January 7-10.

Isom, M.P., 1975. "The Theory of Sound Radiated by a Hovering Transonic Helicopter Blade," Polytechnic Institute of New York, Report POLY-AE/AM 75-4, May.

Iversen, J.D., 1976. “Correlation of Turbulent Trailing Vortex Decay Data," J. of Aircraft, 13 (5), pp. 338-342.

Johnson, W., 1971. "A Lifting Surface Solution for Vortex-Induced Airloads," AIAA J., 9 (4).

Johnson, W., 1971. "Application of a Lifting-Surface Theory to the Calculation of Helicopter Airloads," American Helicopter Soc. Forum. 
Johnson, W., 1980. Helicopter Theory. New York, Dover Publications.

Kalkhoran, I.M. and Wilson, D.R., 1992. "Experimental investigation of the parallel vortex airfoil interaction at transonic speeds," AIAA Journal, 30 (8), pp. 2087-2092.

Katz, J. and Plotkin, A., 2006. Low-Speed Aerodynamics, $2^{\text {nd }}$ edition, New York, Cambridge University Press.

Kitaplioglu, C., and Caradonna, F.X., 1994. "Aerodynamics and Acoustics of Blade Vortex Interaction Using an Independently Generated Vortex," American Helicopter Soc. Aeromechanics Specialist Conference, San Francisco, CA, Jan. 19-21.

Kitaplioglu, C., Caradonna, F.X., and Burley, C.L., 1997. "Parallel Blade-Vortex Interactions: An Exeperimental Study and Comparison with Computation," J. of the American Helicopter Soc., 42 (1), pp. 272-281.

Kokkalis, T. and Galbraith, R.A. McD., 1986. "Description of and Preliminary Results From a New Blade-Vortex Interaction Test Facility," $12^{\text {th }}$ European Rotorcraft Forum, Garmish, Germany, Sept. 22-25.

Kuibin, P.A. and Okulov, V.L., 1996. "One-dimensional solutions for a flow with a helical symmetry," Thermophysics and Aeromechanics, 3(4), pp. 335-339.

Lamb, H., 1932. Hydrodynamics, Cambridge University Press, Cambridge, UK, pp. 592-593, 668-669.

Landgrebe, A.J., 1988. "Overview of Helicopter Wake and Airloads Technology," $2^{\text {nd }}$ International Conference on Rotorcraft Basic Research, College Park, MD, Feb. 16-18.

Landgrebe, A.J., and Johnson, B.V., 1974. "Measurement of Model Helicopter Rotor Flow Velocities with a Laser Doppler Velocimeter," J. of the American Helicopter Soc., 19 (3), pp. 39-43.

Lee, S. and Bershader, D., 1994. "Head-on parallel blade-vortex interaction," AIAA Journal, 32 (1), pp. 16-22.

Lee, S., 1994. "Reduction of blade-vortex interaction noise through porous leading edge," AIAA Journal, 32 (3), pp. $480-488$.

Lee, Y-L., and Baeder, J.D., 2002. "High-Order Overset Method for Blade Vortex Interaction," AIAA Paper 2002-0559, $40^{\text {th }}$ AIAA Aerospace Sciences Meeting and Exhibit, Reno, NV, Jan. 14-17.

Leibovich, S., 1978. “The structure of vortex breakdown,” Annu. Rev. Fluid Mech., 10, pp. 221-246.

Leibovich, S., 1984. "Vortex stability and breakdown: survey and extension," AIAA Journal, 22, pp. 1192-1206.

Leishman, J.G. and Bagai, A., 1991. "Rotor Wake Visualization in Low Speed Forward Flight," AIAA Paper 91 3232, $9^{\text {th }}$ AIAA Applied Aerodynamics Conference, Baltimore, MD, Sept. 23-25.

Leishman, J.G. and Bagai, A., 1998. "Challenges in Understanding the Vortex Dynamics of Helicopter Rotor Wakes," AIAA J., 36 (7), pp. 1130-1140.

Leishman, J.G., 1996. "Seed Particle Dynamics in Tip Vortex Flows," J. of Aircraft, 33 (4), pp. 823-825.

Leishman, J.G., 1997. "Unsteady Aerodynamics of Airfoils Encountering Traveling Gusts and Vortices," J. of Aircraft, 34 (6), pp. 719-729.

Leishman, J.G., 1999. “Acoustic Focusing Effects During Parallel and Oblique Blade Vortex Interaction,” J. 
Sound and Vibration, 221 (3), pp. 415-441.

Leishman, J.G., 2006. Principles of Helicopter Aerodynamics, $2^{\text {nd }}$ edition. New York, Cambridege University Press.

Leishman, J.G., Baker, A.M., and Coyne, A.J., 1995. "Measurements of Rotor Tip Vortices Using ThreeComponent Laser Doppler Velocimetry," J. of the American Helicopter Soc., 41 (4), pp. 342-353.

Leonard, A., 1974. "Energy Cascade in Large-Eddy Simulation of Turbulent Fluid Flows," Adv. Geo-Physics, $18 \mathrm{~A}, \mathrm{pp} .237$.

Lessen, M., Singh, P.J. and Paillet, F., 1974. "The stability of a trailing line vortex. Part I: Inviscid theory," $J$ Fluid Mech., 63, pp.753-763.

Leverton, J.W., 1968. "Helicopter Noise - Blade Slap. Part 1: Review and Theoretical Study," NASA CR 1221, October.

Leverton, J.W., and Taylor, F.W., 1966. "Helicopter Blade Slap," J. of Sound and Vibration, 4 (3).

Lin, S.Y. and Chen, Y.F., 1997. "Numerical study of head-on blade-vortex interaction noise," $35^{\text {th }}$ AIAA Aerospace Sciences Meeting \& Exhibit, Reno, NV, January.

Lorber, P.F., Stauter, R.C., Hass, R.J., Torok, M.S., and Kohlhepp, F.W., 1994. "Techniques for Comprehensive Measurement of Model Helicopter Rotor Aerodynamics," $50^{\text {th }}$ Annual Forum of the American Helicopter Soc., Washington DC, May 11-14.

Lowson, M.V., 1992. "Progress Towards Quieter Civil Helicopters," Aeronautical J., 96, pp. 209-223.

Lowson, M.V., 1996. "Focusing of Helicopter BVI Noise," J. of Sound and Vibration, 190 (3), pp. 477-484.

Mahalingam, R., 1999. "Structure of the Near Wake of a Rotor in Forward Flight and its Effect on Surface Interactions," Ph.D. Dissertation, School of Aerospace Engineering, Georgia Institute of Technology, June.

Mahalingam, R., and Komerath, N.M., 1998. "Measurements of the Near Wake of a Rotor in Forward Flight," AIAA Paper $98-0692,36^{\text {th }}$ Aerospace Sciences Meeting and Exhibit, Reno, NV, Jan. 12-15.

Malakiel, E., Levinski, V. and Cohen, J., 1999. "The evolution of a localized vortex disturbance in external shear flows. part 2. comparison with experiments in rotating shear flows," Journal of Fluid Mechanics, 397, pp. 351380.

Mamou, M., Khalid, M. and Xu, H., 2001. "Unsteady flows past two airfoils in tandem and airfoil-vortex interaction," Canadian Aeronautics and Space Journal, 47 (4), pp. 357-366.

Martin, P.B. and Leishman, J.G., 2002. "Trailing Vortex Measurements in the Wake of a Hovering Rotor with Various Tip Shapes," $58^{\text {th }}$ Annual Forum of the American Helicopter Soc. International, Montreal, Canada, June 11-13.

Martin, P.B., Bhagwat, M.B., and Leishman, J.G., 1999. "Strobed Laser Sheet Visualization of a Helicopter Rotor wake," J. of Flow Visualiztion and Image Processing, 7 (1), pp. $31-50$.

Martin, P.B., Pugliese, G., and Leishman, J.G., 2001. "High Resolution Trailing Vortex Measurements in the Wake of a Hovering Rotor," J. of the American Helicopter Soc., 48 (1), pp. 39-52. 
Martin, R.M., Elliott, J.W., and Hoad, D.R., 1986. "Experimental and Analytical Predictions of Rotor Blade Vortex Interaction," Journal of the American Helicopter Society, 31 (4), October.

McAlister, K.W., 1996. "Measurements in the Near wake of a Hovering Rotor," AIAA Paper 96-1958, 27 th AIAA Fluid Dynamics Conference, New Orleans, LA, June 18-20.

McAlister, K.W., Schuler, C.A., Branum, L., and Wu, J.C., 1995. "3-D Wake Measurements near a Hovering Rotor for Determining Profile and Induced Drag," NASA TP 3577.

McCorskey, W.J. and Goorjian, P.M., 1983. "Interactions of Airfoils with Vertical Gusts and Concentrated Vortices in Unsteady Transonic Flow," AIAA Paper 83-1691, $16^{\text {th }}$ Fluid and Plasma Dynamics Conference, Danvers, MA, July 12-14.

McCorskey, W.J., 1985. "The Effects of Gusts on the Fluctuating Airloads in Transonic Flow," J. of Aircraft, 22 (3), pp. 236-243.

McCorskey, W.J., 1995. "Vortex wakes of Rotorcraft," AIAA Paper 95-0530, 33 ${ }^{\text {rd }}$ AIAA Aerospace Sciences Meeting and Exhibit, Reno, NV, Jan. 9-12.

McCorskey, W.J., and Baeder, J.D., 1985. "Some Recent Advances in Computational Aerodynamics for Helicopter Applications," NASA TM-86777.

McCorskey, W.J., and Goorjian, P.M., 1983. "Interactions of Airfoils with Vertical Gusts and Concentrated Vortices in Unsteady Transonic Flow," AIAA Paper 83-1691, $16^{\text {th }}$ AIAA Fluid and Plasma Dynamics Conference, Danvers, MA, July 12-14.

Meneveau, C., 1996. “A Lagrangian Dynamic Subgrid-Scale Model of Turbulence," J. Fluid Mechanics, 319, pp. 353.

Menter, F.R., 1994. "Two-Equation Eddy-Viscosity Turbulence Models for Engineering Applications," AIAA Journal, 32(8), pp. 1589-1605.

Mercker, E. and Pengel, K., 1992. "Flow Visualization of Helicopter Blade Tip Vortices," $18^{\text {th }}$ European Rotorcraft Forum, Avignon, France, Sept. 15-18.

Newman, B.G., 1959. "Flow in a Viscous Trailing Vortex," Aeronautical Quarterly, 1, May, pp. 167-188.

Norberg, C., 1985. "Interaction between Freestream Turbulence and Vortex Shedding For a Single Tube In CrossFlow," Journal of Wind Engineering and Industrial Aerodynamics, 23.

Ogawa, A, 1993. Vortex Flow, CRC Series on Fine Particle Science and Technology, CRC Press Inc., Chapter 12.

Oseen, C.W., 1911. "Uber Wirdelbewegung in einer reibenden Flussigkeit," Ark. Mat. Astron. Fys., 7, pp. 14-21.

Pegg, R.J., 1979. "A summary and Evaluation of Semi-empirical Methods for the Prediction of Helicopter Rotor Noise," NASA TM-80200, December.

Piomelli, U., 2001. "Large-Eddy and Direct Simulation of Turbulent Flows," 9e Conference Annuelle de la Societe Canadienne de CFD, Montreal, Canada.

Raffel, M., Richard, H., Ehrenfried, K., der Wall, B.V., Burley, C., Beaumier, P., McAlister, K.and Pengel, K., 
2004. "Recording and evaluation methods of piv investigations on a helicopter rotor model," Experiments in Fluids, 36, pp. 146-156.

Rahier, G., 1997. "Modeling of airfoil-vortex interaction and application to a helicopter; contribution to bladevortex interaction noise prediction." PhD thesis, Office National d'Etudes et de Recherches Aerospatiales.

Ramasamy, M. and Leishman, J.G., 2003. "The Interdependence of straining and Viscous Diffusion Effects on Vorticity in Rotor Flow Fields," J. of Aircraft, 41 (5), pp. 1014-1024.

Renzoni, P. and Mayle, R.E., 1989. "Incremental Force and Moment Coefficients for a Parallel Blade-Vortex Interaction," AIAA Journal, 29 (1), pp. 6-13.

Renzoni, P., 1987. "Discrete Vortex Modeling of a Blade-Vortex Interaction," PhD thesis, Resselaer Polytechnic Institute.

Ribera, M., and Celi, R., 2004. "Simulation Modeling of Unsteady Maneuvers Using a Time Accurate Free Wake," $60^{\text {th }}$ Annual Forum of the American Helicopter Soc., Baltimore, MD, June 7-10.

Ringler, T.D., George, A.R., and Steele, J.B., 1991. "The Study of Blade-Vortex Interaction Sound Generation and Directivity," American Helicopter Soc. International Technical Specialist Meeting, Valley Forge, PA, Oct. 15-17.

Rorke, J.B., and Moffitt, R.C., 1977. "Measurement of Vortex Velocities Over a Wide Range of Vortex Age, Downstream Distance, and Freestream Velocity," NASA CR 145213.

Rorke, J.B., Moffitt, R.C., and Ward, J.F., 1972. "Wind tunnel Simulation of Full-Scale Vortices," 28 th Annual Forum of the American Helicopter Soc., Washington DC, May.

Rott, N., 1958. "On the viscous core of a line vortex," Z Angew Math Phys., 9, pp. 543-553.

Rule, J.A. and Bliss, D.B., 1998. "Predicition of Viscous trailing Vortex Structure from Basic Loading Parameters," J. of the American Helicopter Soc., 30 (3), pp. 17-28.

Saffman, P.G. and Baker, G.R., 1979. "Vortex interactions," Annu. Rev. Fluid Mech., 11, pp. 95-122.

Schlegel, R.G., King, R., and Mull, H., 1966. "Helicopter Rotor Noise Correlation and Propagation," USAAVLABS TR 66-4, October.

Schlichting, H., 1955. Boundary Layer Theory, Pergamon Press Ltd.

Schmitz, F.H. and Sim, B.W., 2000. "Radiation and directionality characteristics of advancing side blade-vortex interaction (BVI) noise," in $6^{\text {th }}$ IAA/CEAS Aeroacoustics Conference, Lahaina, Hawaii, June.

Schmitz, F.H. and Yu, Y.U., 1986. "Helicopter Impulsive Noise: Theoretical and Experimental Status," J. of Sound and Vibration, 109 (3), pp. 361-422.

Schmitz, F.H., 1991. "Rotor Noise," Aeracoustics of Flight Vehicles: Theory and Practice, Vol. 1, Chap. 2, NASA Reference Publication 1258.

Schmitz, F.H., 1998. "Reduction of Blade Vortex Interaction (BVI) Noise through X-Force Control," J. of the American Helicopter Soc., 43 (1), pp. 14-24.

Scully, M.P., 1975. "Computation of Helicopter Rotor Wake Geometry and Its Influence on Rotor Harmonic Airloads," Massachusetts Institute of Technology, ASRL TR 178-1. 
Scully, M.P., and Sullivan, J.P., 1972. "Helicopter Rotor wake Geometry and Airloads and Development of Laser Doppler Velocimeter for Use in Helicopter Rotor Wakes," Massachusetts Institute of Technology, ASRL TR 179.

Seath, D.D., Kim, J.M., and Wilson, D.R., 1987. "An Investigation of the Parallel Blade-Vortex Interaction in a Low Speed Wind-Tunnel," AIAA Paper 87-1345, $19^{\text {th }}$ Fluid Dynamics, Plasma Dynamics and Lasers Conference, Honolulu, HI, June 8-10.

Seath, D.D., Kim, J.M., and Wislon, D.R., 1989. "Investigation of the Parallel Blade-Vortex Interaction at Low Speed," Journal of Aircraft, 26 (4), pp. 328-333.

Seelhorst, U., Beesten, B., and Butefisch, K.A., 1994. "Flowfield Investigation of a Rotating Helicopter Rotor Blade by Three-Component Laser Doppler Velocimetry," $75^{\text {th }}$ AGARD Fluid Dynamic Panel Symposium, Berlin, Germany, October.

Seelhorst, U., Raffel, M., Willert, C., Vollmers, K.A., Butefisch, K.A., and Kompenhans, J., 1996. "Comparison of Vortical structures of a Helicopter Rotor Model Measured by LDV and PIV," $22^{\text {nd }}$ European Rotorcraft Forum, Brighton, UK, Sept. 17-19.

Shockey, G.A., Williamson, J.W. and Cox, C.R., 1976. "Helicopter Aerodynamic and Structural Loads Survey," $32^{\text {nd }}$ Annual National Forum of the American Helicopter Society, May.

Shtym, A.N., 1985. "Aerodynamics of cyclone-vortex chambers (in Russian)," Far-Eastern University, Vladivostok.

Sim, B. W-C. and George, A.R., 1996. "The Propagation of Caustics in Rotorcraft Blade-Vortex Interaction Noise," AIAA Paper 96-1737, $2^{\text {nd }}$ AIAA/CEAS Aeroacoustics Conference, State College, PA, May 6-8.

Sim, B. W-C., George, A.R., and Yen, S.J., 1995. "Blade-Vortex Interaction Noise Studies Using the Trace Mach Number," American Helicopter Soc. Aeromechanics Specialist Conference, Bridgeport, CT, Oct. 11-13.

Sim, B. W-C., Leishman, J.G., Strawn, R.C., and George, A.R., 1997. "Analytical and Computational Investigations of Oblique Blade-Vortex Interaction Generated Noise," AIAA Paper 97-1706, $3^{\text {rd }}$ AIAA/CEAS Aeroacoustics Conference, Atlanta, GA, May 12-14.

Sim, B.W. and Schmitz, F.H., 2000. "Blade vortex interaction (BVI) noise: Retreating side characteristics, sensitivity to chordwise loading and unsteady aerodynamics," American Helicopter Society (AHS) Aeromechanics Specialists Meeting, Atlanta, Georgia, November.

Simons, I.A., Pacifico, R.R., and Jones, J.P., 1966. "The Movement, Structure and Breakdown of Trailing Vortices from a Rotor Blade," CAL/USAAVLABS Symp. On Aerodynamic Problems Associated with V/STOL Aircraft, Vol. 1-Propeller and Rotor Aerodynamics, Buffalo, NY, June 22-24.

Singh, R. and Baeder, J.D., 1996. "On the Significance of Transonic Effects on Aerodynamics and Acoustics of Blade Vortex Interaction," AIAA Paper 96-1696, $2^{\text {nd }}$ AIAA/CEAS Aeroacoustics Conference, Monterey, CA, Aug. 9-11.

Singh, R. and Baeder, J.D., 1997. "The Direct Calculation of Indicial Lift Response of a Wing Using Computational Fluid Dynamics," J. of Aircraft, 35 (4), pp. 465-471.

Singh, R. and Baeder, J.D., 1997. "Generalized Moving Gust Response Using CFD with Application to AirfoilVortex Interaction," AIAA Paper 97-2208, $15^{\text {th }}$ AIAA Applied Aerodynamics Conference, Atlanta, GA, June 23- 
25.

Smagorinsky, J., 1963. "General Circulation Experiments with the Primitive Equations I: The Basic Experiment," Monthly Weather Review, 91, pp. 99.

Smith, D. E. and Sigl, D., 1995. "Helicopter Rotor Tip Shapes for Reduced Blade Vortex Interaction - An Experimental Investigation," AIAA Paper 95-0192, January.

Spalart, P. R., 1998. “Airplane trailing vortices,” Annual Revue Fluid Mechanics, 30, pp. 107-138.

Spall, R.E. and Gatski, T.B., 1991. "A Computational Study of the Topology of Vortex Breakdown,“ Mathematical and Physical Science, 435 (1894), pp. 321-337.

Squire, H.B., 1965. "The Growth of a Vortex in Turbulent Flow," Aeronautical Quarterly, 16. Aug., pp. 302-306.

Srinivasan, G.R. and McCorskey, W.J., 1987. "Numerical Simulations of Unsteady Airfoil Interactions," Vertica, $11(1 / 2)$, pp. 3-28.

Srinivasan, G.R., 1986. "Aerodynamics of two-dimensional blade-vortex interaction," AIAA Journal, 24 (10), pp. $1569-1576$.

Sternfeld, H., Bobo, C., Carmichael, D., Fukushima, T., and Spencer, R., 1972. “An Investigation of Noise Generation on a Hovering Rotor, Part ll," Boeing Vertol Company, Report D210-10550-1, November.

Straus, J., Renzoni, P. and Mayle, R.E., 1990. "Airfoil pressure measurements during a blade vortex interaction and a comparison with theory," AIAA Journal, 28 (2), pp. 222-228.

Strawn, R.C., 1997. "New Computational Methods for the Prediction and Analysis of Helicopter Noise," J. of Aircraft, 34 (5), pp. 665-672.

Sullivan, B.M., Edwards, B.D., Brentner, K.S., and Booth, E.A., Jr. 2002. "A Subjective Test of Modulated Blade Spacing for Helicopter Main Rotors," AHS International $58^{\text {th }}$ Annual Forum and Technology Display, Montreal, Canada, June 11-13.

Sullivan, J.P., 1973. "An Experimental Investigation of Vortex Rings and Helicopter Rotor Wakes Using a Laser Doppler Velocimeter," Massachusetts Institute of Technology Aerophysics Laboratory, Technical Report 183, MIT DSR No. 80038.

Sullivan, R.D., 1959. "A two-cell vortex solution of the Navier-Stokes equations," J. Aerosp. Sci., 26, pp. 767770 .

Surendraiah, M., 1970. “An Experimental Study of Rotor Blade-Vortex Interaction,” NASA CR-1573.

Swanson, A.A., 1993. "Application of the Shadowgraph Flow Visualization Technique to a Full-Scale Helicopter Rotor in Hover and Forward Flight," AIAA Paper 93-3411-CP, $11^{\text {th }}$ AIAA Applied Aerodynamics Conference, Monterey, CA, Aug. 9-11.

Tang, L. and Baeder, J.D., 1999. "Improved Euler simulation of Hovering Rotor Tip Vortices with Validation," $55^{\text {th }}$ Annual Forum of the American Helicopter Soc. International, Montreal, Canada, May 25-27.

Tangler, J. L., 1978. "Experimental Investigation of the Subwing Tip and Its Vortex Structure," NASA CR-3058, November. 
Tangler, J.L., 1977. "Schlieren and noise studies of rotors in forward flight," in $33^{\text {rd }}$ American Helicopter Society forum, Washington, DC.

Tavoularis, S., 2008. Theory of Turbulence, Ottawa. University of Ottawa.

Theodore, C., and Celi, R., 1998. "Flight Dynamic Simulation of Hingeless Rotor Helicopters Including a Maneuvering Free wake Model," $54^{\text {th }}$ Annual Forum of the American Helicopter Soc. International, Washington DC, May 20-22.

Thomson, T.L., Komerath, N.M., and Gray, R.B., 1988. "Visualization and Measurement of the Tip Vortex Core of a Rotor Blade in Hover," J. of Aircraft, 25 (2), pp. 1113-1121.

Tung, C., Pucci, S.L., Caradonna, F.X., and Morse, H.A., 1983. "The Structure of Trailing Vortices Generated by Model Helicopter Rotor Blades," Vertica, 7 (1), pp. 33-43.

Tung, C., Yu, Y.H. and Low, S.L., 1996. "Aerodynamic aspects of blade-vortex interaction (BVI)," 27th Fluid Dynamics Conference, New Orleans, LA, June.

Vasiliev, O.F., 1958. "The Foundations of Mechanics of Helical and Circulating Flows (in Russian)," Gosenergoizdat, Moscow-Leningrad.

Vatistas, G.H., Kozel, V., and Mih, W.C., 1991. "A Simpler Model for Concentrated Vortices," Experiments in Fluids, 11, pp. 73-76.

Wagner, C., Huttl, T. and Sagaut, P., 2007. Large-Eddy Simulation for Acoustics, New York, Cambridge University Press.

White, F.M., 2007. Fluid Mechanics, $6^{\text {th }}$ ed., McGraw-Hill.

White, F.M., 2006. Viscous Fluid Flow, $3^{\text {rd }}$ edition, New York, McGraw Hill.

White, R. P. Jr., Balcerak, J. C. amd Pegg, R. J., 1975. "Summary of Results Indicating the Beneficial Effects of Rotor Vortex Modification," Proceedings of the National Symposium on Helicopter Aerodynamic Efficiency, Hartford, Conn., March 6-7.

Widnall, S., 1971. "Helicopter Noise Due to Blade-Vortex Interaction," J. of the Acoustical Soc. Of America, 50 (1), Pt. 2, pp. 354-365.

Wong, O. D., 2001. "Formation and Evolution of Tip Vortices of an Isolated Rotor in Forward Flight," Ph.D. Dissertation, School of Aerospace Engineering, Georgia Institute of Technology, December.

Wong, S.H., Papadakis, M., Nizampatham, L.S. and Hoffmann, K.A., 2000. "Computational investigation of blade vortex interaction noise," in 38th AIAA Aerospace Sciences Meeting and Exhibit, Reno, NV, January.

Wong, Y.G.A, 2003. "A Study on the Use of Volterra Integrals for the Identification of Rotor Blade-Vortex Interaction," M.A.Sc. Thesis, Carleton University.

Yu, H. Y., 2002. "Rotor blade-vortex interaction noise," Progress in Aerospace Sciences, 36, pp. 97-115.

Yu, Y.H., 1996. "Miss distance for rotor blade-vortex interaction noise reduction," in $2^{\text {nd }}$ Aeroacoustics Conference, State College, PA, May. 
Yu, Y.H., 2000. "Rotor blade-vortex interaction noise," Progress in Aerospace Sciences, 36, pp. 97-115.

Yu, Y.H., Gmelin, Y.B., Heller, H., Phillippe, J.J., Mercker, E., and Preisser, J.S., 1994. "Higher Harmonic Control Aeroacoustics Test- The Join German/French/US HART Project," Proceedings of $20^{\text {th }}$ European Rotorcraft Forum, Amsterdam, Netherlands, Oct 4-7.

Zdravkovich, M. M., 2003. Flow around Circular Cylinders, Vol. 2: Applications. Toronto, Oxford University Press. 


\section{Appendix A: Theory Associated with the Computational Methods Commonly Used for Analyzing the Airfoil-Vortex Interaction}

\section{A.1 Shear Stress Transport (SST) Model: Theory}

Manipulation of the $k-\varepsilon$ and $k-\omega$ models results in the following relations for the SST model:

$$
\begin{aligned}
\frac{\partial k}{\partial t}+u_{i} \frac{\partial k}{\partial x_{i}} & =\tau_{i j} \frac{\partial u_{i}^{\prime}}{\partial x_{j}}-\beta^{*} k \omega+\frac{\partial}{\partial x_{i}}\left[\left(v+\sigma_{k} v_{e}\right) \frac{\partial k}{\partial x_{i}}\right] \\
\frac{\partial \omega}{\partial t}+u_{i} \frac{\partial \omega}{\partial x_{i}}= & \alpha \frac{\omega}{k} \tau_{i j} \frac{\partial u_{i}^{\prime}}{\partial x_{j}}-\beta \omega^{2}+\frac{\partial}{\partial x_{i}}\left[\left(v+\sigma_{\omega} v_{e}\right) \frac{\partial \omega}{\partial x_{i}}\right] \\
& +2\left(1-F_{1}\right) \frac{\sigma_{\omega 2}}{\omega} \frac{\partial k}{\partial x_{i}} \frac{\partial \omega}{\partial x_{i}}
\end{aligned}
$$

where

$$
\begin{gathered}
\tau_{i j}=v_{T}\left(2 S_{i j}-\frac{2}{3} \frac{\partial u_{k}}{\partial x_{k}} \delta_{i j}\right)-\frac{2}{3} k \delta_{i j} \\
S_{i j}=\frac{1}{2}\left(\frac{\partial u_{i}}{\partial x_{j}}+\frac{\partial u_{j}}{\partial x_{i}}\right)
\end{gathered}
$$

In order to determine the any constant, which shall be represented as $\Phi$ i.e. $\left(\sigma_{k}, \ldots\right)$, in Equation ( A.1 ) and Equation ( A.2 ), a blending process may be used. If $\Phi_{l}$ were to represent any constant in the original $k-\omega$ model, i.e. $\left(\sigma_{k 1}, \ldots\right)$ and $\Phi_{2}$ were 
to represent any constant in the transformed $k-\varepsilon$ model, i.e. $\left(\sigma_{k 2}, \ldots\right)$, then the constants $\Phi$ in the SST model may be determined through

$$
\Phi=F_{1} \Phi_{1}+\left(1-F_{1}\right) \Phi_{2}
$$

where, $F_{1}=\tanh \left(\arg _{1}^{4}\right)$ and the argument, $\arg _{1}$, is given by

$$
\arg _{1}=\min \left[\max \left[2 \frac{\sqrt{k}}{0.09 y \omega} ; \frac{500 \nu}{y^{2} \omega}\right] ; \frac{4 \sigma_{\omega 2} k}{C D_{k \omega} y}\right]
$$

where $y$ is the distance closest to the wall and $C D_{k \omega}$ represents the cross-diffusion term and is given by

$$
C D_{k \omega}=\max \left[2 \frac{\sigma_{\omega 2}}{\omega} \frac{\partial k}{\partial x_{i}} \frac{\partial \omega}{\partial x_{i}} ; 10^{-20}\right]
$$

The constants which are required in Equations ( A.1) through ( A.7) are as follows:

$$
\begin{gathered}
\sigma_{k 1}=0.85 ; \sigma_{\omega 1}=0.5 ; \beta_{1}=0.075 ; \alpha_{1}=\beta_{1} / \beta^{*}-\sigma_{\omega 1}\left(\kappa^{2} / \sqrt{\beta^{*}}\right) \\
\sigma_{k 2}=1.0 ; \sigma_{\omega 2}=0.856 ; \beta_{2}=0.0828 ; \alpha_{2}=\beta_{2} / \beta^{*}-\sigma_{\omega 2}\left(\kappa^{2} / \sqrt{\beta^{*}}\right)
\end{gathered}
$$

with $\beta^{*}=0.09$ and $\kappa=0.41$ (Menter, 1994).

The model which was employed prior to the SST was referred to as the Baseline (BSL) model. This model used the same approach as the SST, but failed to predict the onset/amount of flow separation under adverse pressure gradients and did not account for the transport of the shear stress which results in an overprediction of the eddy viscosity (Menter, 1994). Consequently, the SST model (i.e. Equation ( A.1 ) and Equation ( A.2 )), uses a further constraint which is imposed through the limiting of the eddy viscosity, $v_{e}$, through the following relationship 


$$
v_{e}=\frac{a_{1} k}{\max \left[a_{1} \omega ; \Omega F_{2}\right]}
$$

the constant, $a_{1}$, in the above relation is normally taken to be 0.31 and $\Omega$ represents the absolute value of the vorticity within the flow field (Menter, 1994). The second function, $F_{2}$, is given by

$$
F_{2}=\tanh \left(\arg _{2}^{2}\right)
$$

where the argument, $\arg _{2}$, is given by

$$
\arg _{2}=\max \left[2 \frac{\sqrt{k}}{0.09 y \omega} ; \frac{500 v}{y^{2} \omega}\right]
$$

\section{A.2 Subgrid Scale (SGS) Modeling: Theory}

\section{Smagorinsky Model:}

The Smagorinsky model is the most commonly used model in LES to date (Wagner et al., 2007). This model is an eddy viscosity model, which is based on the increase in transport and dissipation which results from the effects of the SGS stress (Smagorinsky, 1963). In laminar flows, the aforementioned increases are a result of the viscosity in laminar flows (Smagorinsky, 1963). The Smagorinsky SGS model may be written as

$$
\tau_{i j}-\frac{1}{3} \delta_{i j} \tau_{k k}=-v_{T}\left(\frac{\partial \bar{u}_{l}}{\partial x_{j}}+\frac{\partial \bar{u}_{j}}{\partial x_{i}}\right)=-2 v_{T} \overline{S_{l j}}
$$

where $v_{T}$ is referred to as the eddy viscosity, and

$$
\overline{S_{\imath \jmath}}=\frac{1}{2}\left(\frac{\partial \bar{u}_{l}}{\partial x_{j}}+\frac{\partial \bar{u}_{j}}{\partial x_{i}}\right)
$$


The Smagorinsky model is known as an eddy viscosity type model, as it assumes that the small eddies remove energy from the flow through a dissipative process (Wagner et al., 2007). A weak point in this model results from the possibility of backscatter (which is the reintroduction of energy from the small scales to the large scales) may occur (Wagner et al., 2007). In turbulent flows, energy is introduced in the largest scales and transferred to the small scales until the viscous damping effects dominate (Wagner et al., 2007). As a result, in the regions where the viscous effects are dominant, the turbulent energy of the flow is damped out through the transfer of kinetic energy into heat (i.e. internal energy), which is referred to as the energy cascade (Wagner et al., 2007). Between these two subscales, there exists a region where there is neither significant amounts of production or dissipation of energy which is referred to as the inertial subrange (Wagner et al., 2007). In the inertial subrange, the only active mechanism is the inviscid one and it is assumed that the transfer of energy occurs from the large scales to the small ones (Piomelli, 2001). Figure A.1 depicts the kinetic energy as wavelength (or size) of the eddies. 


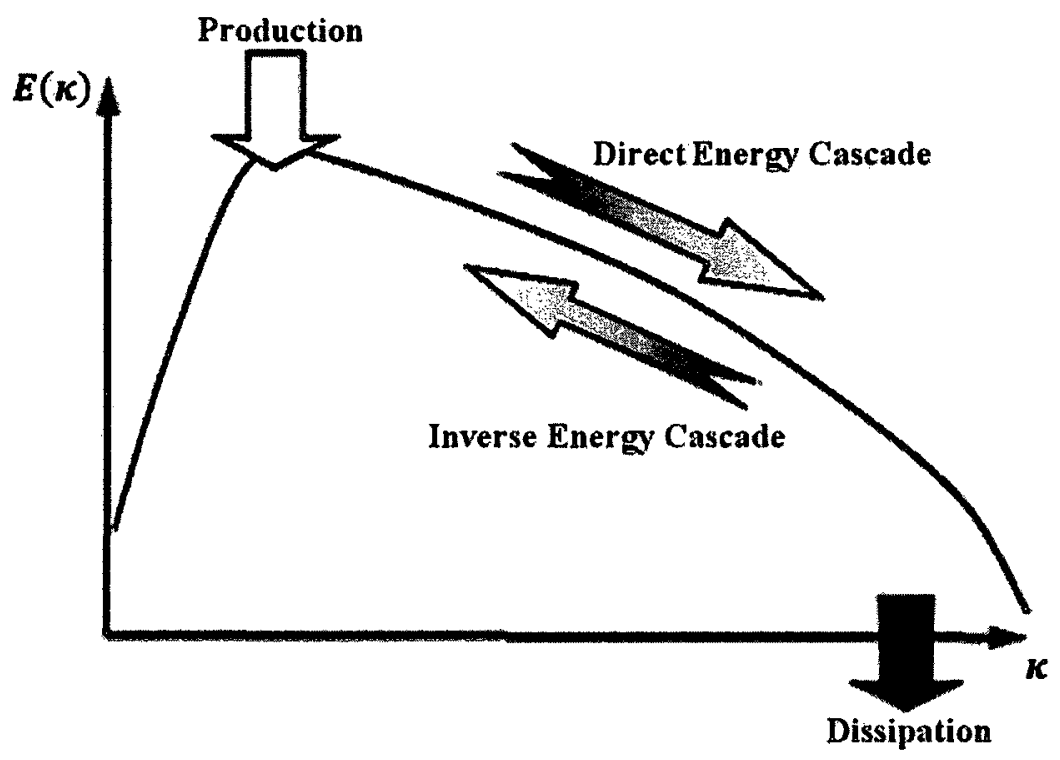

Figure A.1: Kinetic Energy Transfer in Isotropic Turbulence (Wagner et al., 2007)

The mechanism which is responsible for this energy transfer is referred to as the advection term in the Navier-Stokes equations (Wagner et al., 2007) which allows the rate of energy transfer to the smaller scales to be evaluated (Piomelli, 2001). The magnitude of the contribution to the kinetic energy by the advection term is

$$
\frac{1}{2} \frac{\partial}{\partial x_{j}}\left(u_{i} u_{j}\right)
$$

Noting that the energy is introduced at the largest scale, one may estimate the dissipation rate, $\varepsilon$, through

$$
\varepsilon=\frac{U_{L}^{3}}{L}
$$

where $U_{L}$ represents the velocity scale of the largest eddies and, $L$, is the integral length scale of the turbulent flow (refer to Section A.3 for further discussion of this term). If it is assumed that the largest SGS are much larger than the viscous scales, then the following may be obtained 


$$
\varepsilon \approx \frac{U_{S G S}^{3}}{\Delta}
$$

where $U_{S G S}$ represents the velocity scale of the smallest eddies and, $\Delta$, is the length of the largest SGS eddies (it also represents the length scale associated with the filter) (Piomelli, 2001).

A consequence of the energy transfer from the large scales to the small scales is that once the energy is lost by the large eddies to the small eddies through the cascade it may not be recovered (Piomelli, 2001). SGS represents this transfer of energy as an effective viscous dissipation in the Smagorinsky model (Wagner et al., 2007). Since the smallest resolved scales (of size delta), are most significant for the SGS model, the effective viscous dissipation is estimated as

$$
\varepsilon \approx \frac{v_{T} U_{S G S}^{2}}{\Delta^{2}}
$$

Equating Equation ( A.15) and Equation ( A.16) leads to a conclusion of proportionality, i.e. $v_{T} \propto \Delta U_{S G S}$ and as a result it can be shown that

$$
v_{T} \approx \Delta^{4 / 3} L^{-1 / 3} U_{L}
$$

Equation ( A.17) may be used to show that

$$
U_{L} \approx L\left(2 \overline{S_{l \jmath}} \cdot \overline{S_{l \jmath}}\right)^{\frac{1}{2}}=L|\bar{S}|
$$

where, $|\bar{S}|=\left(\overline{S_{\imath \jmath}} \cdot \overline{S_{\imath \jmath}}\right)^{\frac{1}{2}}$ (Wagner et al., 2007). Making use of Equation ( A.18 ) in Equation ( A.17), the following relation may be derived

$$
v_{T}=C_{S}^{2} \Delta^{4 / 3} L^{2 / 3}|\bar{S}|
$$


where, $C_{s}$, is the Smagorinsky constant and is introduced to ensure equality (Wagner et al., 2007). The Smagorinsky parameter may be determined through

$$
C_{s} \approx \frac{1}{\pi}\left(\frac{2}{3 K_{o}}\right)^{3 / 4}=0.18
$$

where $K_{o}=1.41$ (Wagner et al., 2007). It is worth noting that most theories which provide estimates for $C_{s}$ are valid only for isotropic turbulence (Wagner et al., 2007). The Smagorinsky constant may also be derived as a function of the Reynolds number along with other non-dimensional parameters for different flow regimes, however, it is generally accepted that the estimate presented in Equation ( A.20) is generally valid (Wagner et al., 2007). Another difficulty arises when the integral length scale, $L$, is to be determined (Wagner et al., 2007). As a result of the added degree of complexity, the following simplification is often employed (Wagner et al., 2007). This simplification is that $\Delta^{4 / 3} L^{-1 / 3} \rightarrow \Delta^{2}$, which leads to the following more common form of the Smagorinsky eddy viscosity model (Wagner et al., 2007)

$$
v_{T}=\left(C_{s} \Delta\right)^{2}|\bar{S}|
$$

The filter width, $\Delta$, is often determined through $\left(\Delta_{1} \Delta_{2} \Delta_{3}\right)^{1 / 3}$ or $\left(\Delta_{1}^{2}+\Delta_{2}^{2}+\Delta_{3}^{2}\right)^{1 / 2}$ (Piomelli, 2001), where $\Delta_{i}$ represents the filter width in the i-th coordinate direction. Employing an appropriate length scale ensures the damping is irrelevant (Wagner et al., 2007). 
Despite being the most commonly used SGS model, several weak points are inherent within the Smagorinsky model (Wagner et al., 2007). One major problem is that in the case of non-homogenous wall bounded flows (i.e. near the surface of the walls), the model does not damp out the eddy viscosity enough in order to allow the viscosity effects to dominate (Wagner et al., 2007). As a result, when the analysis reaches the viscous sublayer, the approximation which was made in the Smagorinsky model becomes questionable as the grid point (i.e. $\Delta$ ) will be in or near the viscous sublayer (Wagner $e t$ al., 2007). For this reason, Van Driest recast the eddy viscosity in the near wall region for the reduced growth of the small scales near the wall in the following form (Wagner et al., 2007)

$$
v_{T}=\left[C_{s} \Delta\left(1-e^{-\left(y^{+} / 25\right)}\right)\right]^{2}|\bar{S}|
$$

A second issue is that the Smagorinsky model assumes that the energy transfer from the large scales to the small scales is a diffusive process. In other words, there is no mechanism that transfers energy from the small scales to the large ones with no resulting backscatter (Wagner et al., 2007). The resolved transport energy equation is given as

$$
\frac{\partial \bar{q}^{2}}{\partial t}+\frac{\partial\left(\bar{q}^{2} u_{j}\right)}{\partial x_{i}}=\frac{\partial}{\partial x_{i}}\left[-2 \bar{p} \cdot \bar{u}_{\jmath}-2 \tau_{i j} \bar{u}_{l}+\frac{1}{R e} \frac{\partial \bar{q}^{2}}{\partial x_{j}}\right]-\frac{2}{R e} \frac{\partial \bar{u}_{l}}{\partial x_{j}} \frac{\partial \bar{u}_{l}}{\partial x_{j}}+2 \tau_{i j} \overline{S_{l j}}
$$

where $\bar{q}^{2}=\bar{u}_{l} \cdot \bar{u}_{l}$ and the one half of the last term may be defined as (Wagner et al., 2007)

$$
\varepsilon_{S G S}=\tau_{i j} \overline{S_{l j}}
$$


The transfer of energy between the large and small scales is represented by $\varepsilon_{S G S}$ and is referred to as the subgrid scale dissipation. When $\varepsilon_{S G S}<0$, the resolved energy decays with respect to the interaction between the large and small scales (i.e. forward scatter). However, when $\varepsilon_{S G S}>0$, the resolved energy grows with respect to the interaction between the large and small scales (i.e. back scatter). In the case of the Smagorinsky model, Equation ( A.20) and Equation ( A.23) may be used to illustrate that $\varepsilon_{s G s}<0$, as a result there is no backscatter from the Smagorinsky model (Wagner et al., 2007).

In order to model more complex or higher Reynolds number flows, the use of a more accurate DNS model should be employed, however, a modified Smagorinsky model may be employed or a dynamic subgrid scale model may also be employed in order to overcome the issues with the Smagorinsky model (Piomelli, 2001). This dynamic subgrid scale model shall now be discussed.

\section{Dynamic Subgrid Scale Model:}

As a result of the issues with the Smagorinsky model, a new approach which is referred to as the dynamics subgrid scale model has been investigated. This approach attempts to calculate the Smagorinsky parameter interactively during the run time rather than setting this parameter as a constant at the beginning of the simulation (Piomelli, 2001). The procedure employed in the dynamic subgrid scale model involves the application of a second filter, which is referred to as a test filter, and is denoted by a tilde $(\sim)$ to the filtered Navier-Stokes equation (see Equation ( 3.7 )) (Wagner et al., 2007). The addition of the test filter results in the following modified form of the filtered Navier-Stokes equations 


$$
\frac{\partial \overline{\tilde{u}_{l}}}{\partial t}+\frac{\partial}{\partial x_{j}}\left(\overline{\tilde{u}}_{i} \cdot \overline{\tilde{u}_{J}}\right)=-\frac{\partial \overline{\tilde{P}}}{\partial x_{i}}-\frac{\partial T_{i j}}{\partial x_{j}}+\frac{1}{\operatorname{Re}} \frac{\partial^{2}}{\partial x_{j}^{2}}\left(\overline{\tilde{u}}_{\imath}\right)
$$

where $T_{i j}=\overline{\widetilde{u_{\imath}} \widetilde{u_{\jmath}}}-\overline{\tilde{u}_{\imath}} \cdot \overline{\tilde{u}_{\jmath}}$ (Wagner et al., 2007). If the Smagorinsky model is employed as the SGS model, the filter and test filter levels may be represented as (Wagner et al., 2007)

$$
\begin{aligned}
& \tau_{i j}-\frac{1}{3} \delta_{i j} \tau_{k k} \approx-2 C_{s} \Delta^{2}|\bar{S}| \overline{S_{\imath \jmath}}=2 C_{s} B_{i j} \\
& T_{i j}-\frac{1}{3} \delta_{i j} T_{k k} \approx-2 C_{s} \tilde{\Delta}^{2} \mid \overline{\tilde{S}} \overline{\widetilde{S_{l j}}}=2 C_{s} A_{i j}
\end{aligned}
$$

where, $C_{s}$ is used as opposed to $C_{s}^{2}$ in the eddy viscosity term, and $\Delta$ and $\tilde{\Delta}$ represent the width of the first filter and test filter respectively (Wagner et al., 2007). The SGS stress at the end of the first/test filters are related to the resolved turbulent stress through an algebraic identity known as the Germanos identity (Germano et al., 1991), i.e.

$$
L_{i j}=T_{i j}-\widetilde{\tau_{\imath j}}
$$

If the stresses are modeled, then Germanos identity is no longer satisfied (Germano et al., 1991). Hence the residual equation is obtained as

$$
E_{i j}=L_{i j}+2 C_{s} A_{i j}-2 C_{s} \widetilde{B_{\imath \jmath}}
$$

The dynamic subgrid scale model is based on minimizing the residuals, and on assuming that the Smagorinsky parameter, $C_{s}$, is known (denoted by $C_{s}^{*}$ ) in the third term on the right hand side of Equation ( A.29 ) (Germano et al., 1991). This assumption allows Equation (A.29) to be rewritten as 


$$
E_{i j}=L_{i j}+2 C_{s} A_{i j}-2 C_{s}^{*} \widetilde{B_{l j}}
$$

and the Smagorinsky parameter $C_{s}^{*}$ in Equation ( A.30) is then approximated through a Taylor series expansion of $C_{s}$ (Germano et al., 1991). Figure A.2 depicts how the dual filters account for the transfer of energy.

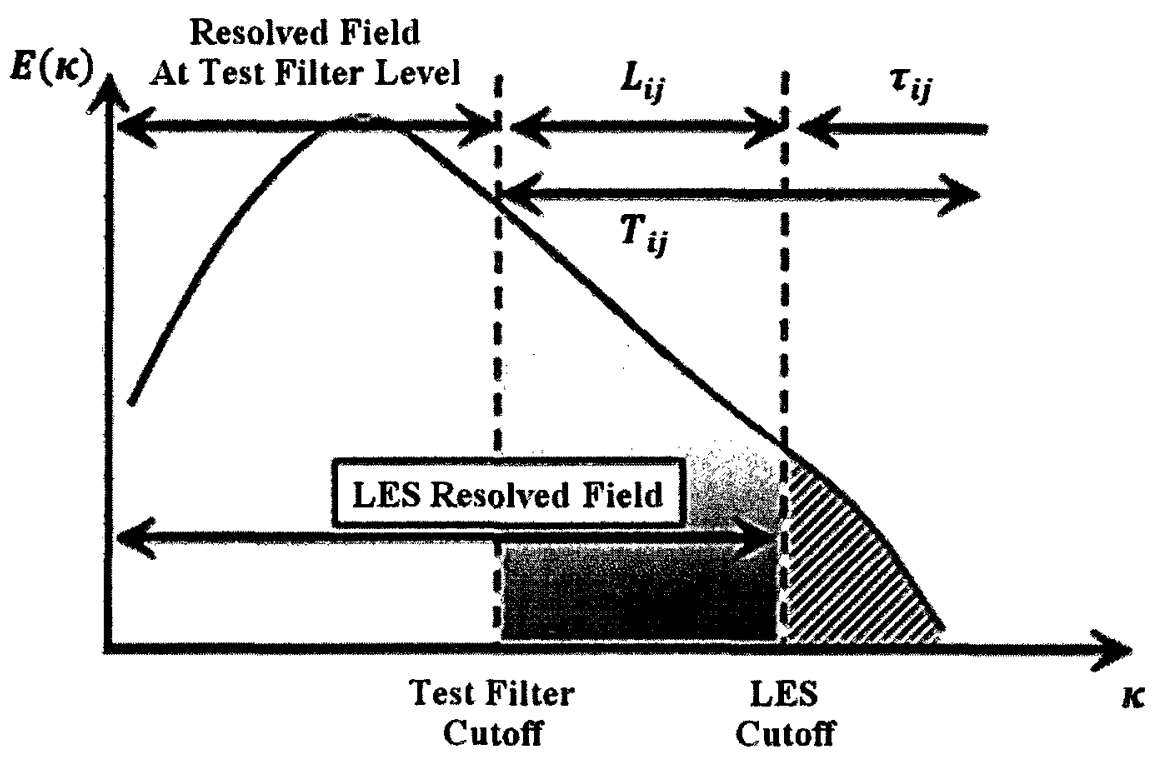

Figure A.2: Depiction of the Two-Level Filtering Procedure (Wagner et al., 2007)

One of the advantages of the dynamics subgrid scale model is that no parameters need be determined a priori to the simulation. Also, the dynamic SGS model has the capability to reduce the eddy viscosity in the laminar flow regime. There still remain unresolved issues with the dynamic SGS model however. One of which is that in the derivation of the model, it is assumed that the Smagorinsky parameter is independent of the filter width (Wagner et al., 2007). It has been shown, however, through DNS data from isotropic turbulence, that the instantaneous values of the Smagorinsky parameter vary significantly when compared for two different filter widths (Meneveau, 1996). A 
further issue with the dynamic SGS is that it allows for $C_{S}$, and hence the eddy viscosity to take on negative values. This leads to numerical instabilities, despite it also allowing for back scatter to be accounted for in the model (Wagner et al., 2007).

A more accurate approach to LES, despite the additional computational complexity is DNS and shall now be addressed in full.

\section{A.3 Direct Numerical Simulation (DNS)}

As the name implies, DNS is a straight forward approach to the numerical simulation of turbulent flows. The range of scales present in turbulent flows is a common function of the Reynolds number and in DNS all of these scales must be resolved, from the dissipative scales of order $\eta$ to integral scales of order $L$ (Piomelli, 2001), through the direct solution of the Navier-Stokes equations with no averaging/approximation applied. In the case of incompressible fluids with uniform density, the non-dimensionalized Navier-Stokes equations, in standard tensor notation, may be represented as:

$$
\begin{gathered}
\frac{\partial u_{i}}{\partial x_{i}}=0 \\
\frac{\partial u_{i}}{\partial t}+\frac{\partial}{\partial x_{j}}\left(u_{i} u_{j}\right)=-\frac{\partial P}{\partial x_{i}}+\frac{1}{R e} \frac{\partial^{2}}{\partial x_{j}^{2}}\left(u_{i}\right)
\end{gathered}
$$

where $P$ represents the non-dimensional pressure and is defined as $P=p / \rho$ and $R e$ is the Reynolds number and is defined as $R e=U L / \nu$. The Reynolds number in Equation ( A.32 ) is defined by the characteristic (integral) length scale, $L$, the characteristic velocity, $U$, (the root-mean-square (RMS) of the velocity) and the kinematic viscosity, $v$ (Wagner et al., 2007). 
The length scale of the domain must be at least as large as the largest turbulent eddy in order to ensure the significant structures are captured. A common measure is the integral scale, $L$, of turbulence which is defined as the distance over which the velocity remains correlated. This approach assumes that the velocities within a turbulent eddy are highly correlated and there is no correlation between velocities in different eddies (Piomelli, 2001). The characteristic turbulent length representing the mean of all existing eddies is applied as theoretically, the largest eddy approaches an infinite size, this length scale is thus defined as:

$$
L_{i}=\frac{1}{\overline{u_{\imath}^{\prime} u_{j}^{\prime}}} \int_{0}^{\infty} R_{i j} d r_{i}
$$

where $R_{i j}$ represents the correlation tensor of the velocity components, and is given as

$$
R_{i j}(x, t, r, \tau)=\overline{u_{l}^{\prime}(x, t) u_{j}^{\prime}(x+r, t+\tau)}
$$

where, $r$, is the distance from the current point, $x$, to the neighbouring point, $x+r$. As a result of the nature of turbulent flows (i.e. dominated by eddies (vortices)), correlations are useful tools to analyze the statistical nature of the flow (Piomelli, 2001).

A DNS simulation must capture all the entire range of spatial and temporal scales of the turbulent flow field. The spatial scales of the turbulence, from the smallest dissipative scales (i.e. the Kolmogorov microscales) up to the integral scale, $L$, associated with the motions containing most of the kinetic energy, must be resolved in the computational mesh. The Kolmogorov microscales are the Kolmogorov length scale, $\eta$, and Kolmogorov time scale, $\tau$, respectively. These scales may be determined by the 
dissipation rate, $\varepsilon$, which represents the transformation of kinetic energy into thermal energy and the kinematic viscosity, $v$. The Kolmogorov length scale is given by $\eta=$ $\left(v^{3} / \varepsilon\right)^{1 / 4}$ and the Kolmogorov time scale is represented as $\tau=(\nu / \varepsilon)^{1 / 2}$. It is worth noting that the integral scale generally depends on the spatial scale of the boundary conditions. In order to satisfy the spatial resolution requirements, the number, $N$, of points along a given mesh in one direction with increments, $\Delta x$, must be $N \Delta x>L$. As a result, the integral scale is contained within the computational domain. The other constraint placed on the spatial resolution of a DNS simulation to ensure that the Kolmogorov length scale is resolved is that $\Delta x \leq \eta$. In other words, the computational domain must be large enough to contain the largest scales, $L$, of motion; whereas the grid resolution must be fine enough to resolve the smallest scales, $\eta$, of motion giving the number of grid points required as proportional to $L / \eta \propto R e^{3 / 4}$. Since $\varepsilon \approx U^{3} / L$, the previous relations demand that, for a three-dimensional DNS, simulation requires a number of mesh points, $N^{3}$, which satisfy $N^{3} \geq R e^{9 / 4}$. The time scale of the smallest eddies also provides a bound for the maximum time step, $\Delta t \propto R e^{1 / 2}$, and it must also be ensured that the time step is small enough such that a fluid particle will traverse a mere fraction of the mesh spacing, $\Delta x$, in each time step. That is, $C=\frac{u \Delta t}{\Delta x}<1$ where $C$ is the Courant number. The total time interval simulated is generally proportional to the Kolmogorov time scale (i.e. $\tau=L / U$ ) combination of the previous time step relations; this proportion yields that the number of time steps must be proportional to $L /(C \eta)$. Analyzing the required number of grid points and time steps required for a DNS 
simulation yields the cost of a calculation in the order of $R e^{11 / 4}$. This approximate dependence of the computational time on the Reynolds number illustrates the impracticality of DNS for high Reynolds number flows (Piomelli, 2001).

Despite the large computational cost of DNS, it is a useful tool to validate turbulence models. Most approaches to numerical simulations of high Reynolds number flows are based on insight gained from a physical description of turbulence provided through DNS simulations for low Reynolds number flows. The irregularity of a turbulent flow is one of its most important characteristics. Despite the difficulty in completely describing turbulence as a function of time and space coordinates, the average values of the flow field (i.e. pressure and velocity) may be described. Numerous experiments/observations showed that these averages exist as at any given point in the turbulent domain:

1) a distinct pattern is repeated more or less regularly in time, and

2) a distinct pattern is repeated more or less regularly in space

These results lead to the conclusion that, on average, turbulence has the same overall structure in the domain (Wagner et al., 2007). Further, the details of the small scale eddies are not of interest for many engineering applications (Piomelli, 2001). 


\section{Appendix B: Viscous Blade Tip Vortices: Additional Models}

\section{B.1 Rankine Vortex}

The Rankine vortex (Lamb, 1932) is the simplest viscous tip vortex model. It is a model of a cylindrical vortex of finite core radius, $r_{c}$, and constant vorticity, $\omega$, which is present only within the vortices core. The Rankine vortex, shown in Figure B.1, is more realistic than the model provided in Section 4.2, namely an infinitely thin vortex filament.

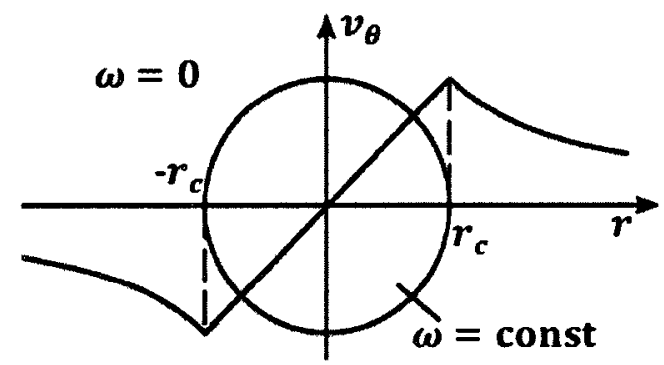

a)Swirl Velocity Profile

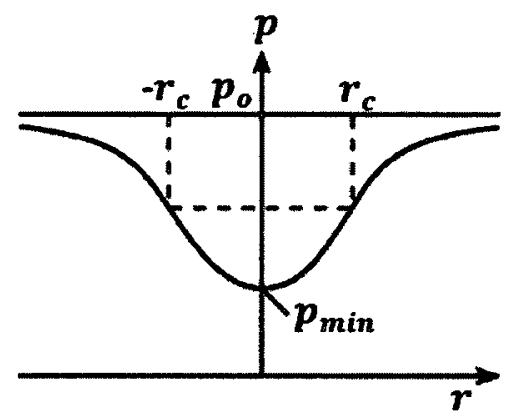

b)Static Pressure Profile

Figure B.1: a) Swirl Velocity Profile and, b) Static Pressure Distribution of a Rankine Vortex (Alekseenko et al., 2003)

In the Rankine vortex model, the core is modeled as a solid body rotation. In the outer region, the velocity decreases hyperbolically with distance as a potential vortex.

$$
v_{\theta}(r)=\left\{\begin{array}{c}
\frac{\Gamma}{2 \pi r_{c}} \frac{r}{r_{c}}, \text { if }, 0 \leq r \leq r_{c} \\
\frac{\Gamma}{2 \pi r_{c}} \frac{r_{c}}{r}, i f, r \geq r_{c}
\end{array}\right.
$$


The linearity of the profile illustrated in Figure B.1a indicates a solid body rotation of the fluid within the vortex core, at an angular velocity of

$$
\Omega=\frac{\Gamma}{2 \pi r_{c}^{2}}
$$

The vorticity distribution for the Rankine vortex is represented by

$$
\omega=\left\{\begin{array}{cc}
\frac{\Gamma}{2 \pi r_{c}^{2}}, & r<r_{c} \\
0, & r \geq r_{c}
\end{array}\right.
$$

Equation ( B.3 ) implies that the vorticity is all contained within the vortex core. In other words, there is no vorticity in the potential region, as shown in Figure B.1a. The break in the vorticity field at the edge of the vortices core region is a result of the vorticity jump within the model. However, confining the vorticity within the vortex core radius as with solid body rotation has been found to be a poor representation of a real vortex. Furthermore, since the tangential velocity at a given radius from the vortex is determined only by the vorticity inside the radius. Having some vorticity outside of the core radius implies the peak velocity is less than $\frac{\Gamma}{2 \pi r_{c}}$. It has been found that the maximum tangential velocity of a real vortex is much less than the value of $\frac{\Gamma}{2 \pi r_{c}}$. Therefore, a substantial fraction of the vorticity is actually outside of the core radius.

The radial distribution of static pressure for a blade tip vortex is characterized by a drastic decrease in the vortex core and is generally given by

$$
p=p_{\infty}+\rho \int_{\infty}^{r} \frac{v_{\theta}^{2}}{r} d r
$$


Employing the combination of Equation ( B.1) and Equation ( B.3 ) in Equation ( B.4 ) yields the radial static pressure distribution for the Rankine vortex, which is given by

$$
p(r)=\left\{\begin{array}{c}
p_{\infty}-\rho \frac{\omega^{2} r_{c}^{4}}{8 r^{2}}, r>r_{c} \\
p_{\infty}-\rho \frac{\omega^{2} r_{c}^{2}}{4}+\rho \frac{\omega^{2}}{8} r^{2}, r<r_{c}
\end{array}\right.
$$

as is illustrated in Figure B.1b.

The minimum pressure occurs at the vortex axis, i.e. when $r=0$, and is given by

$$
p_{\min }=p_{\infty}-\rho \frac{\omega^{2} r_{c}^{2}}{4}=p_{\infty}-\rho \frac{\Gamma^{2}}{4 \pi^{2} r_{c}^{2}}
$$

the pressure at the core radius is given by

$$
p-p_{\min }=\frac{1}{2}\left(p_{\min }-p_{\infty}\right)
$$

In contrast, for an infinitely thin vortex filament of strength, $\Gamma$, the pressure at the axis, i.e. at $r=0$, tends diverge.

Despite exhibiting key features of a viscous vortex, the Rankine vortex produces unrealistically high values of the peak swirl velocity compared to measurements. However, the Rankine vortex model remains the most popular model as it reflects the main features of concentrated vortices regardless of its shortcomings.

To fix the vorticity jump in the Rankine model, Scully proposed an alternative laminar model of the viscous tip vortex, as follows.

\section{B.2 Scully Vortex}

Scully (1975) found that confining the vorticity within the vortex core radius as a solid body rotation is an inaccurate representation of a real vortex. Since the tangential 
velocity at a given radius from the vortex is determined only by the vorticity inside the radius, vorticity outside of the core radius suggests that the peak velocity is less than $\frac{\Gamma_{v}}{2 \pi r_{c}}$. The maximum tangential velocity of a real vortex is much less than the value of $\frac{r_{v}}{2 \pi r_{c}}$. This implies that a substantial fraction of the vorticity is actually outside of the core radius. Therefore, Scully (1975) suggested that, based on measured velocity distributions of vortices from fixed wings, the circulation distribution of a real vortex is given as

$$
\gamma=\Gamma \frac{r^{2}}{r^{2}+r_{c}^{2}}
$$

which has a corresponding vorticity distribution of

$$
\omega=\frac{\Gamma}{2 \pi r_{c}^{2}} \frac{1}{\left[1+\left(r / r_{c}\right)^{2}\right]^{2}}
$$

where, $r$ is the distance from the line vortex. Equation ( B.9) implies that half the vorticity is contained inside the core radius, and the other half is within the potential region. The induced velocity distribution may therefore be represented by

$$
v_{\theta}(r)=\frac{\Gamma}{2 \pi}\left[\frac{r}{\left(r_{c}^{2}+r^{2}\right)}\right]
$$

which effectively moves the vortex peak further away, i.e. to a location given by

$$
\left(y_{v}\right)_{e q}=\sqrt{y_{v}^{2}+r_{c}^{2}}
$$

The maximum tangential velocity is now $\frac{r_{v}}{4 \pi r_{c}}$ at $r=r_{c}$ which is exactly half the value which was obtained using a solid body rotation inside the viscous core of the vortex, which is more appropriate based on vortex velocity distribution measurements. 
Several other tip vortex models employ exponential profiles for the swirl velocity distribution as well. One of which involves an extension of the Lamb-Oseen model to the third-direction, i.e. the addition of an axial velocity distribution as well as providing the radial velocity component. This model shall be referred to as the Newmans model.

\section{B.3 Newmans Three-Dimensional Extension of the Lamb-Oseen Vortex}

Similar to the Lamb-Oseen model, Newman (1959) also derived exponential solutions for the three velocity components in the viscous core based vortex using a simplified Navier-Stokes formulation. The boundary conditions imposed by Newman (1959) first involved the assumption that the vortex generates as a free vortex (i.e. potential vortex of strength $\Gamma$ at $z=0$ ). It diffuses until at large distances the vortex induced velocity goes to zero. Upon application of these boundary conditions, it was determined that the swirl velocity is as per the Lamb-Oseen profile, see Equation ( 4.16), and the axial and radial velocity is given by

$$
\begin{aligned}
& v_{z}(r, z)=\frac{-A}{z} e^{\left(-\alpha\left(r / r_{c}\right)^{2}\right)} \\
& v_{r}(r, z)=\frac{-A r}{2 z^{2}} e^{\left(-\alpha\left(r / r_{c}\right)^{2}\right)}
\end{aligned}
$$

Where $A$ is a constant which may be related to the drag on a lift-generating surface if the vortex is of lift-generation origin, such as blade-vortex interaction.

Another laminar model of a viscous tip vortex is the Gauss vortex, which shall now be detailed. 


\section{B.4 Gauss Vortex}

Hopfinger and van Heijst (1993) developed smooth profiles of vorticity and azimuthal velocity while numerically examining the monopolar vortex instabilitiy. The vorticity and the swirl velocity which were developed are given as

$$
\begin{gathered}
\bar{\omega}=\frac{\omega R}{U}=\left[1-\frac{1}{2} q\left(\frac{r}{R}\right)^{q}\right] e^{\left(-\left(\frac{r}{R}\right)^{q}\right)} \\
\overline{v_{\theta}}=\frac{v_{\theta}}{U}=\frac{1}{2} \frac{r}{R} e^{\left(-\left(\frac{r}{R}\right)^{q}\right)}
\end{gathered}
$$

where, $R$ and $U$ represent the scales of length and velocity respectively and $q$ is a shape factor. If $q$ is set to a value of 2 and a non-dimensional radial coordinate is introduced as $\bar{r}=\sqrt{2}\left(\frac{r}{R}\right)$ then Equation ( B.14) may be expressed as

$$
\omega=\left[1-\frac{1}{2} \bar{r}^{2}\right] e^{-\left(\frac{\bar{r}^{2}}{2}\right)}
$$

The vortex described by Equation ( B.16) is referred to as the Gauss vortex, since the dimensionless stream function, $\bar{\psi}$, is described by the Gauss distribution, i.e.

$$
\bar{\psi}=\frac{1}{2} e^{-\left(\frac{\bar{r}^{2}}{2}\right)}
$$

The expressions defined by Hopfinger and Van Heijst (1993), i.e. Equation ( B.16) and Equation ( B.17 ), are often used for the generalization of experimental data as a result of them making use of the Gaussian distribution.

The following employ the concept of columnar vortices; as a result this topic shall now be introduced. 


\section{B.5 One-Dimensional Columnar Vortices}

Columnar vortices have some vorticity distribution in the axial direction dependent on the radial location as opposed to the axial location in a helical flow. In a one-dimensional helical flow, the streamlines coincide with the vortex lines resulting in a swirling axisymmetric flow. Extending this into three-dimensions leads to the vorticity distribution in the axial direction, which was not present in the models presented above.

In a one-dimensional helical flow, if velocity components depend only on the radial coordinate, and $\lambda=$ const $\neq 0$, then $v_{r}=0$ and from the Navier-Stokes equations in cylindrical coordinates one may conclude that

$$
\frac{1}{v_{\theta}} \frac{\partial v_{z}}{\partial r}=\lambda
$$

defines only sustained helical flow of inviscid fluid in a round tube with a uniform energy distribution.

A simple example of an elementary helical vortex was produced by Vasiliev (1958). Vasiliev (1958) derived a simple distribution for $v_{\theta}$ which is equivalent to the velocity distribution within a Rankine vortex (i.e. Equation ( B.1 )). It is given by $v_{\theta}=\alpha r$ where $\alpha$ is some constant. Using this expression for $v_{\theta}$ and integrating Equation (B.18) gives the axial velocity distribution as $v_{z}=\sqrt{v_{0}^{2}-2 \alpha r^{2}}$ where $v_{0}$ represents the flow at the axis of the vortex thus allowing for a non-uniform radial distribution of the axial component of velocity, $v_{z}$.

Elementary helical vortex structures have been described formally. Some examples include the infinitely thin vortex filament as well as vortex sheets of helical 
vortex filaments. Real blade tip vortices have a finite sized core and are often represented by axisymmetric vortices (or columnar vortices). In contrast with the Rankine vortex, axisymmetric helical vortices created through the superposition of helical vortex filaments, several examples of which are illustrated in Figure B.2, was considered by Kuibin and Okulov (1996).
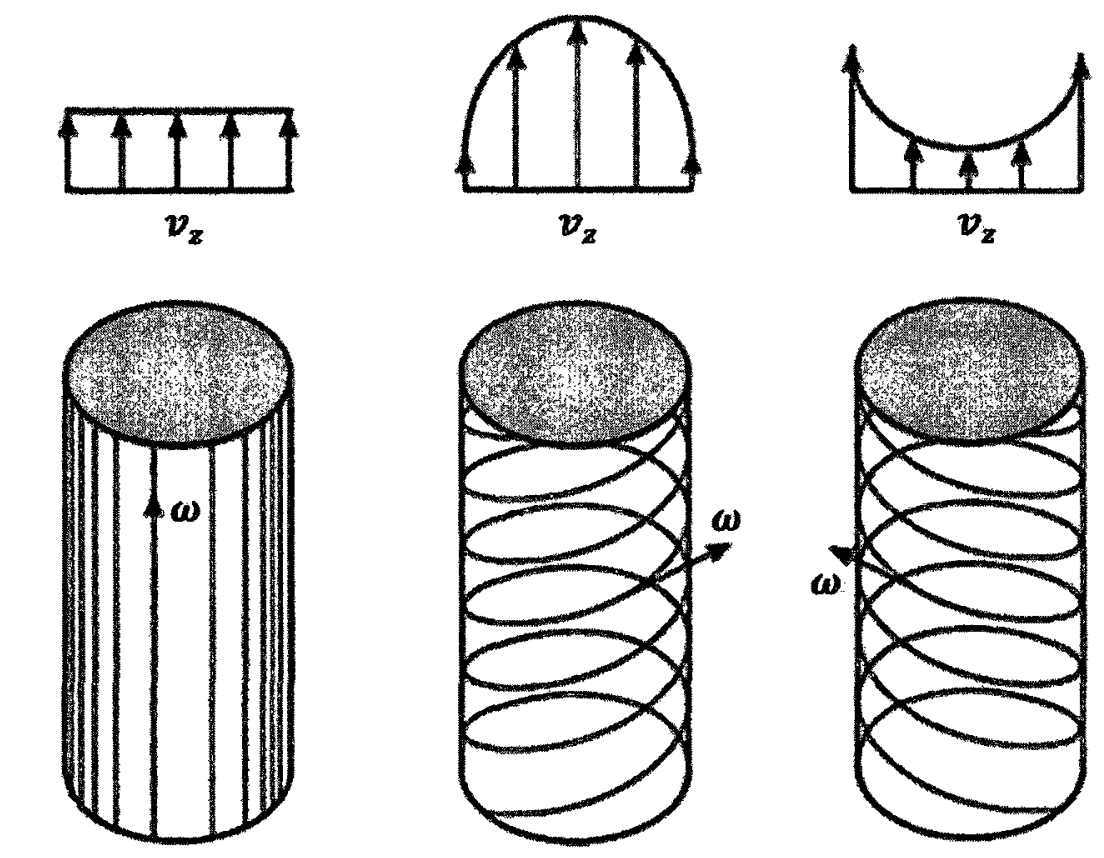

Figure B.2: Schemes of Axisymmetric Helical Vortices (Alekseenko et al., 2003)

If the distribution of filament strengths within the cylindrical region is known as depicted in Figure B.2, the swirl and axial velocity profiles as well as the static pressure distribution in the columnar vortex are given by

$$
\begin{gathered}
v_{\theta}=\frac{1}{r} \int_{0}^{r} \omega_{z}\left(r^{\prime}\right) r^{\prime} d r^{\prime} \\
v_{z}=v_{0}-\frac{1}{l} \int_{0}^{r} \omega_{z}\left(r^{\prime}\right) r^{\prime} d r^{\prime}
\end{gathered}
$$




$$
p=p_{0}+\rho \int_{0}^{r} \frac{v_{\theta}^{2}}{r^{\prime}} d r^{\prime}
$$

where, $v_{0}$ and $p_{0}$ are the velocity and pressure at the tube axis, $r^{\prime}$ represents a change of variable for the integration, $l$ represents the pitch of the vortex in the axial direction and $\rho$ represents the fluid density. If one were to introduce the dimensionless function, $\Phi(r)$, where

$$
\Phi(r)=\frac{2 \pi}{\Gamma} \int_{0}^{r} \omega_{z}\left(r^{\prime}\right) r^{\prime} d r^{\prime}
$$

Then the velocity expressions for columnar vortices, i.e. Equation ( B.19) and Equation (B.20), may be rewritten as

$$
\begin{gathered}
v_{\theta}=\frac{\Gamma}{2 \pi r} \Phi(r) \\
v_{z}=v_{0}-\frac{\Gamma}{2 \pi l} \Phi(r)
\end{gathered}
$$

Furthermore, for columnar vortices it is common to introduce expressions for both the dimensionless vorticity, $\overline{\omega_{z}}$, and the dimensionless pressure drop throughout the vortex, $\Delta \bar{p}$. These are given as

$$
\begin{gathered}
\overline{\omega_{z}}=\frac{\pi \varepsilon^{2}}{\Gamma} \omega_{z} \\
\Delta \bar{p}=\frac{8 \pi^{2} \varepsilon^{2}}{\Gamma^{2}}\left(\frac{p-p_{0}}{\rho}\right)
\end{gathered}
$$

where, $\varepsilon$ is the typical size of the vortex. It is commonly chosen as the viscous core radius of the tip vortex, $r_{c}$ for $\mathrm{R} / \mathrm{C}$ applications. 
Using relations Equation ( B.19) through Equation ( B.26 ), varying models may be used to describe columnar vortices. One such includes the implementation of a uniform distribution of vorticity within the core radius, this representation has been found to be consistent with the Rankine vortex, which was presented in Sub-Section B.1. Another model often used involves the use of a fractional power distribution of vorticity giving a model which coincides with the Scully vortex, as discussed in Sub-Section B.2. Finally, a Gaussian distribution of vorticity within the core produces velocity profiles identical to the Lamb-Oseen model, as seen in Sub-Section 4.3.1. A more general model which has been proposed by Vatistas et al. (1991) shall now be presented and will be referred to within the current work as Vatistas vortex.

\section{B.6 Vatistas Vortex}

A more general series of desingularized velocity profiles for vortices with distribution of the flow was quatitatively given by Vatistas et al. (1991). The swirl velocity profile was obtained by Vatistas et al (1991) through the use of an algebraic relation with an integer exponent, $n(=1, \ldots, \infty)$, which allows different profiles to be obtained. The swirl velocity profile of Vatistas vortex is given as

$$
v_{\theta}(r)=\frac{\Gamma}{2 \pi}\left[\frac{r}{\left(r_{c}^{2 n}+r^{2 n}\right)^{1 / n}}\right]
$$

Several of the previous models may be obtained by implementing different values of the exponent $n$ in Equation ( B.27). For instance, if $n=\infty$ the Rankine vortex velocity profile, given by Equation ( B.1 ), is recovered. Furthermore, if $n=1$ the Scully 
vortex (presented in Sub-Section B.2 as Equation (B.10)) is recovered. Finally, if $n=2$, the Squire vortex given in Sub-Section 4.4.2 is recovered as

$$
v_{\theta}(r)=\frac{\Gamma}{2 \pi}\left[\frac{r}{\sqrt{\left(r_{c}^{4}+r^{4}\right)}}\right]
$$

It is worth noting that the Squire vortex, i.e. if $n=2$ in Equation ( B.27), is an approximation of the Lamb-Oseen profile (refer to Equation ( 4.16 )) which can be obtained through a Taylor series expansion of Equation ( B.27) with $n=2$.

Since Vatistas et al. (1991) assume columnar vortices, the axial and radial velocities given as Equation ( B.12) and Equation ( B.13) in Sub-Section B.3 do not strictly apply to trailing edge vortices. Measurements performed by Vatistas et al. (1991) however, show good correlation with Lamb-Oseen and $n=2$ swirl velocity models. They also confirm the self similar nature of the velocity field surrounding a tip vortex. Slight deviations may be attributed to the presence of extraneous wake vorticity from the inboard part of the blade and other blades within the measurement grid. The axial velocities obtained by Vatistas et al. (1991) have good agreement and are also selfsimilar for each of the Lamb-Oseen and $n=2$ models, and the fluctuations are present as a result of small asymmetries within the tip vortex itself. In this study it was determined that the Scully vortex is a less accurate descriptor of the velocity field present within a rotor blades tip vortex, when compared to both the Lamb-Oseen model and the Vatistas vortex when $n=2$.

Figure B.3 represents a graphical summary of Vatistas vortex with several $n$ values as well as both the Lamb-Oseen vortex and the Rankine vortex. 


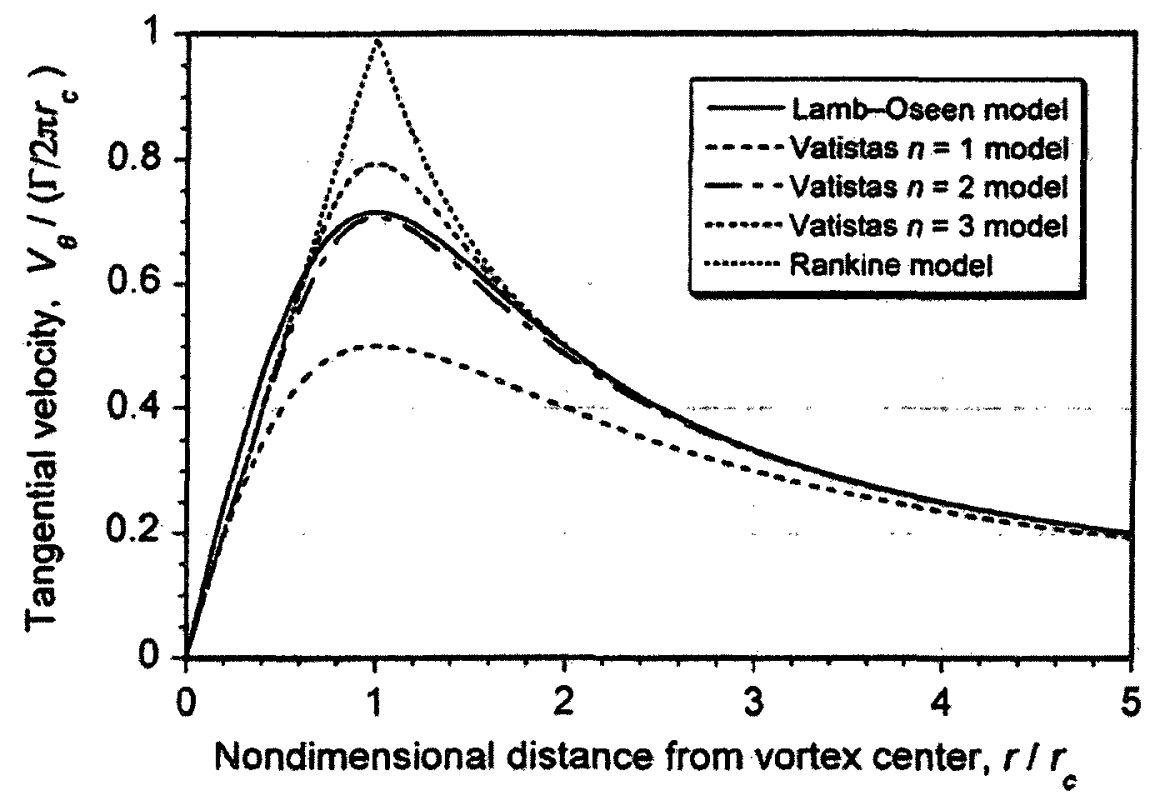

Figure B.2: Two-Dimensional Swirl Velocity Profiles for Numerous Tip Vortex Models (Leishman, 2006)

Another generalization of experimental data was proposed by Leibovich (1978, 1984) from which another laminar description of tip vortices was spawned, namely the Q-vortex, which shall now be addressed.

\section{B.7 Q-Vortex}

In order to describe a flow both before (i.e. while the tip vortex is in its invsicd state) and after vortex breakdown (i.e. when the vortex is in its viscous form), empirical formula are commonly employed, Leibovich (1978, 1984) proposed the following distributions for the swirl velocity and the axial velocity of a tip vortex

$$
\begin{gathered}
v_{\theta}=\frac{K}{r}\left[1-\mathrm{e}^{\left(-\alpha r^{2}\right)}\right] \\
v_{z}=W_{1}+W_{2} \mathrm{e}^{\left(-\alpha r^{2}\right)}=W_{1}+W_{2}-W_{2}\left[1-\mathrm{e}^{\left(-\alpha r^{2}\right)}\right]
\end{gathered}
$$


where, $K, W_{1}, W_{2}$ and $\alpha$ are all empirically determined constants. Here the empirical constants may be related to the physical properties of the tip vortex as vortex as

$$
\begin{gathered}
\Gamma=2 \pi K \\
l=K / W_{2} \\
v_{0}=W_{1}+W_{2} \\
\varepsilon=1 / \sqrt{\alpha}
\end{gathered}
$$

Relations Equation ( B.31) through Equation ( B.34 ) specify the physical sense of the tip vortex and identify a larger class of swirling flows which are induced by columnar helical vortices (which shall be introduced in Sub-Section B.5) with a Gaussian distribution of vorticity within the core.

The expression for the swirl velocity given in Equation ( B.29) corresponds to Burgers vortex (Burgers (1940)) and satisfies the Navier-Stokes equations. The expression for axial velocity, given in Equation ( B.30 ), however differs from Burgers solution since Burgers is only a function of the $z$-coordinate. For the relations corresponding to Burgers vortex refer to Sub-Section B.8.

Equations ( B.29) and ( B.30) were first studied against instability by Lessen $e t$ al. (1974) and were derived from a self similar solution by Batchelor (1964) for a swirling wake. These relations provide great accuracy when describing experimental data and are thus widely used for the processing of swirling flow data. 
Since Leibovich's models, i.e. Equation ( B.29) and ( B.30), are often applied to swirling flow stability, often a simpler one-parameter model based solely on the radial coordinate is used. This model is given by

$$
\begin{gathered}
v_{\theta}=\frac{q}{r}\left[1-\mathrm{e}^{-r^{2}}\right] \\
v_{z}=\mathrm{e}^{-r^{2}}
\end{gathered}
$$

Equation ( B.35 ) and Equation ( B.36) are referred to as the Q-vortex model.

Like the Leibovich vortex the parameters for the Q-vortex in the velocity relations (Equation ( B.35 ) and Equation ( B.36 )) may be related to the physical parameters of the vortex through the following

$$
\begin{gathered}
\Gamma=2 \pi q \\
l=q \\
v_{0}=1
\end{gathered}
$$

which seriously constricts the class of flows for which the Q-vortex may be used in. The velocity profiles described for both Leibovich and the Q-vortex, are both solutions to the equations of ideal fluid motion and satisfactorily describe related experimental data.

The aforementioned vortex deduced by Burgers (1940) shall now be presented to describe a viscous tip vortex produced by a rotor blade.

\section{B.8 Burgers Vortex}

Accounting for viscosity tends to smooth out the peculiarities present in the region of the vortex core in models of infinitely thin vortex filaments as well as other models as well. Since the vortex evolves in time from a potential vortex to one with a 
viscous core, some measure of a time dependent profile must be obtained. In order to compare the time-dependent profiles of vorticity and velocity with experimental data, a scale must or linear measure of a vortex at a given point in time is needed. This scale is given as, $a=2 \sqrt{v t}$, and the profiles are given as

$$
\begin{gathered}
v_{\theta}=\frac{\Gamma}{2 \pi r}\left[1-\mathrm{e}^{\left(-r^{2} / a^{2}\right)}\right] \\
\omega=\frac{\Gamma}{\pi a^{2}} \mathrm{e}^{\left(-r^{2} / a^{2}\right)}
\end{gathered}
$$

Equations ( B.40) and ( B.41) have been found by numerous researchers (Saffman and Baker, 1979; and Hopfinger and van Heijst, 1993) to describe the LambOseen vortex. The vorticity distribution Equation ( B.41) and the azimuthal velocity Equation ( B.40) coincide with the three-dimensional steady state Burgers vortex. This vortex was pioneered by Burgers $(1940,1948)$ and Rott $(1958)$ to describe turbulent swirling flows. Burgers vortex pertains to the class of axisymmetric exact solutions of the Navier-Stokes equations, which are expressed in the form

$$
\begin{gathered}
v_{r}=u(r) \\
v_{\theta}=v(r) \\
v_{z}=w(r)=z f(r)
\end{gathered}
$$

Donaldson and Sullivan (1960) described a family of solutions similar to those obtained by Burgers by assuming that flow was formed in an infinitely long rotating porous tube, with appropriate boundary conditions set at the wall. Sullivan (1959) obtained a boundless solution in space and in a two-cell vortex, by assigning velocity, circulation, and an added constant at infinity. 
Burgers vortex follows from the approach employed by Sullivan, in which a vortex which does not diffuse (i.e. the temporal aspect is removed) shall be addressed. In this case the solution follows a form where the assumed radial and circumferential velocity profiles are given by Equation ( B.42) and Equation ( B.43 ) respectively, and the solution for the axial component of velocity is of the form

$$
v_{z}=\alpha z
$$

where $\alpha$ is a constant. Assuming the solution takes the form of the above three relations, it can be shown the radial and tangential components of velocity are given by

$$
\begin{gathered}
v_{r}=-\alpha(r / 2) \\
\frac{v_{\theta}}{v_{m}}=\frac{v(r)}{\left(r / r_{m}\right)}\left[1-\mathrm{e}^{\left(-1.26\left(r / r_{m}\right)^{2}\right)}\right]
\end{gathered}
$$

where the mean velocity, $v_{m}$, and the mean radius, $r_{m}$, in Equation (B.47) are given by

$$
\begin{gathered}
v_{m}=0.16\left(\Gamma_{v} / \pi\right) \sqrt{\alpha / v}=0.36\left(\Gamma_{v} / \pi r_{m}\right) \\
r_{m}=2.242 \sqrt{v / \alpha}
\end{gathered}
$$

Figure B.4 presents an illustrative comparison of Burgers vortex to the Rankine vortex in order to illustrate how Burgers vortex compares to the previous models which have been provided. Refer to Figure B.3 to see how Rankine's vortex relates to numerous other vortices to obtain a better understanding of the data obtained by Burgers vortex. 


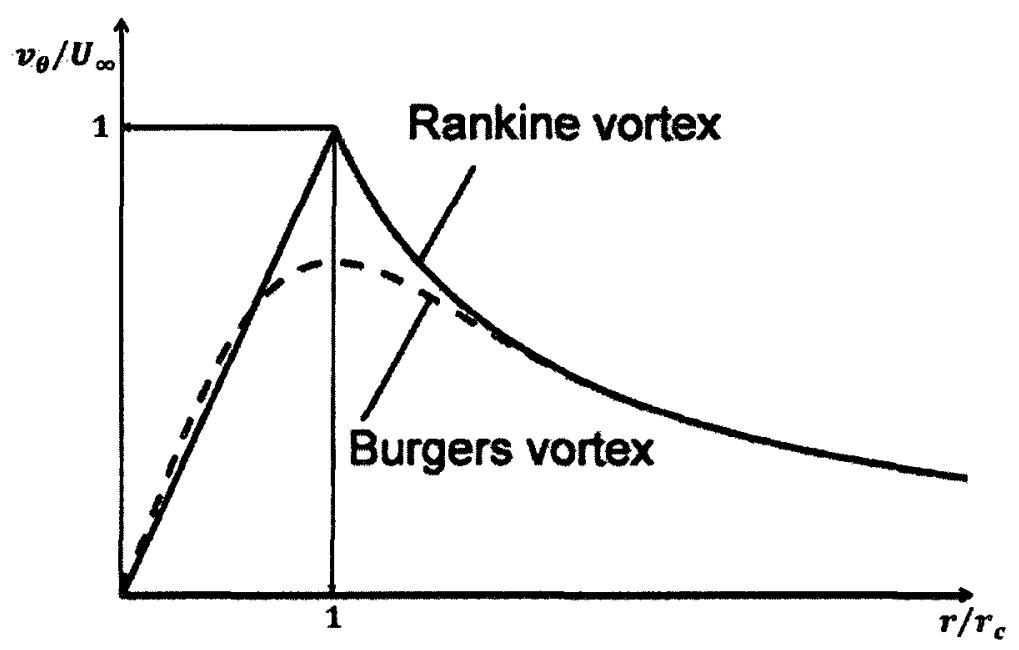

Figure B.4: Swirl Velocity Profiles of Rankine and Burgers Vortices (Alekseenko et al., 2003)

The pressure distribution for the Burgers vortex is given as

$$
p=p_{0}-\frac{1}{2} \rho \alpha^{2}\left(z^{2}+\frac{r^{2}}{4}\right)+\rho \int_{0}^{r} \frac{v_{\theta}^{2}}{r} d r
$$

The existence of a steady state solution for a viscous fluid is accounted for by the fact that the viscous vorticity diffusion is compensated through the radial transferred of vorticity due to the axial straining of the vortex, since $v_{z}=\alpha z$.

The aforementioned solution technique proposed by Sullivan (1959) also obtained another model for the vortex which includes vorticity. This model shall now be presented.

\section{B.9 Sullivans Vortex}

Sullivan (1959) obtained the exact Navier-Stokes solution in the form of a two cell vortex as well, which is given as

$$
v_{r}=-\frac{\alpha r}{2}+\frac{6 v}{r}\left[1-\mathrm{e}^{\left(-\alpha r^{2} / 4 v\right)}\right]
$$




$$
\begin{gathered}
v_{\theta}=\frac{\Gamma}{2 \pi r}\left[\mathrm{H}\left(\frac{\alpha r^{2}}{4 v}\right) / \mathrm{H}(\infty)\right] \\
v_{z}=\alpha z\left[1-3 \mathrm{e}^{\left(-\alpha r^{2} / 4 v\right)}\right] \\
p=p_{0}-\frac{1}{2} \rho\left[\alpha^{2} z^{2}+\frac{\alpha^{2} r^{2}}{4}+36 \frac{v^{2}}{r^{2}}\left[1-\mathrm{e}^{\left(-\alpha r^{2} / 4 v\right)}\right]^{2}\right]+\rho \int_{0}^{r} \frac{v_{\theta}^{2}}{r} d r
\end{gathered}
$$

where

$$
\mathrm{H}(\mathrm{x})=\int_{0}^{x} e^{\left(-t+3 \int_{0}^{t}\left[1-e^{(-s)} / s\right] d s\right)} d t
$$

and $\Gamma$ is the circulation at inifinity, $p_{0}$ is the pressure at $r=z=0$ and $v$ is the kinematic viscosity of the fluid. As $r \rightarrow \infty$, Sullivans vortex exactly coincides with Burgers vortex.

Further investigations related to Sullivans vortex have been performed. BellamyKnights (1970) generalized Sullivans vortex for a time dependent situation, thus obtaining a solution which describes the breakdown of a two cell vortex with time. Also Rott (1958) studied unsteady one-dimensional cell solution. 


\section{Appendix C: The Structure of Blade Tip Vortices}

Blade tip vortices include regions of both laminar and turbulent flow separated by a region of transitional flow, as shown through the observations made by Hoffman and Joubert (1963) and Tung et al. (1983). Figure C.1 represents a flow visualization image of a blade tip vortex with the laminar, transitional and turbulent zones indicated.

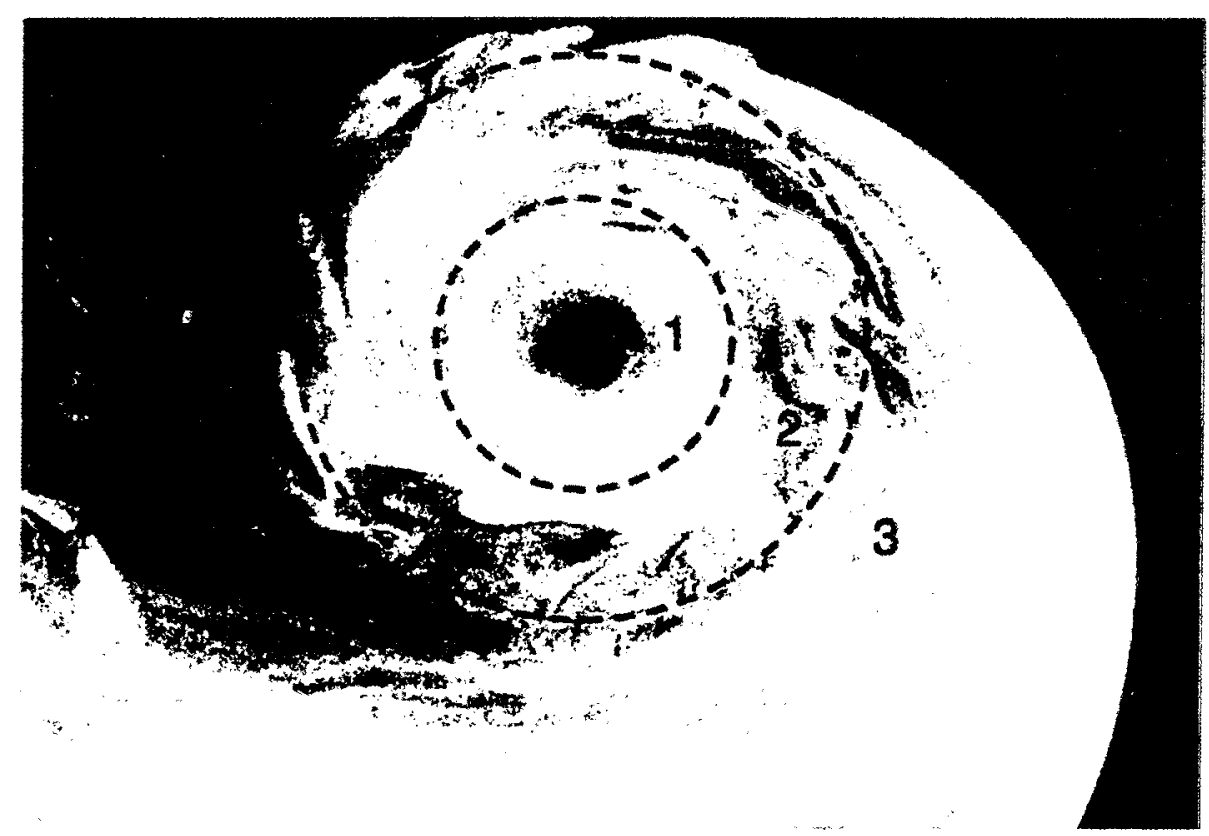

Figure C.1: Smoke Flow Visualization of a Tip Vortex Emanating from a Rotor Blade with Three Regions Indicated (1 - Laminar Region, 2 - Transitional Region, 3 - Turbulent Region) (Leishman, 2006)

From Figure $\mathrm{C} .1$ it can be seen that there is a predominant laminar region near the core region (a region characterized by smooth flow with no interactions between adjacent layers) indicated by 1 . The laminar zone is followed by a transitional region with eddies 
of varying size, marked by 2 . Outside the transitional zone is a highly turbulent region which is denoted by 3 .

Recent support of the multi-zonal structure of tip vortices has been provided by high resolution velocity measurements made with an LDV system (Martin et al., 2001; Martin and Leishman, 2002; and Ramasamy and Leishman, 2003). This differs from previous models as the structure is neither completely laminar, as per the Lamb-Oseen model, nor completely turbulent, as in the Squire or Iversen models. The extent of the 3 regions is affected by $R e_{v}$. Investigations have shown that the effects of flow rotation on the development of turbulence within the vortex play an important role in determining the vortex structure (Hoffman and Joubert, 1963; Cortel and Breidenthal, 1999; and Holzapfel et al., 2001). However, Rayleighs centrifugal instability theory suggests that the vortex will never develop turbulence if the product of the velocity and radial distance increases with the radial coordinate.

Bradshaw (1983) developed an analogy between rotational flows and stratified fluids based on flow rotation. This study resulted in higher speed fluid preferring the external to the vortex while conserving angular momentum (even if the density throughout the vortex is constant). To determine the local strength of the analogous stratification expressed as an equivalent gradient Richardson number, $R i$, an experiment was developed by Bradshaw (1983) using energy principles. The equivalent gradient Richardson number is given as

$$
R i=\left(\frac{2 v_{\theta}}{r^{2}} \frac{\partial\left(v_{\theta} r\right)}{\partial r}\right) /\left(r \frac{\partial\left(v_{\theta} / r\right)}{\partial r}\right)^{2}
$$


Holzapfel et al. (2001) determined that $R i$ comes directly from the turbulent kinetic energy budget equation. Thus, $R i$ is the ratio of the turbulence produced or conserved inside a vortex as a result of buoyancy, to turbulence produced by shearing in the flow (i.e. $R i$ is a ratio of potential energy to kinetic energy in a stratified flow).

Cortel and Breidenthal (1999) and Cortel (2002) used the stratified concept developed by Bradshaw to determine a threshold value of $R i$ as a function of $R e_{v}$. These analyses were based on a "persistence parameter", the ratio of the rotational speed to the translational (or convective) speed of a vortex. If the persistence parameter is high, as it is in most $\mathrm{R} / \mathrm{C}$ wakes, the threshold $R i$ is given by

$$
(R i)_{t h r e s h}=R e_{v}^{1 / 4}
$$

Equation ( C.2 ) implies that the tip vortex will be laminar up to the radial distance where the local gradient $R i$ falls below this threshold value. As a result, any turbulence inside this boundary will either be relaminiarized or suppressed regardless of the eddy size.

An example of how this approach is employed is presented in Figure C.2 which illustrates the variance of $R i$ with radial distance from the center of the vortices core for a vortex Reynolds number of 50,000. 


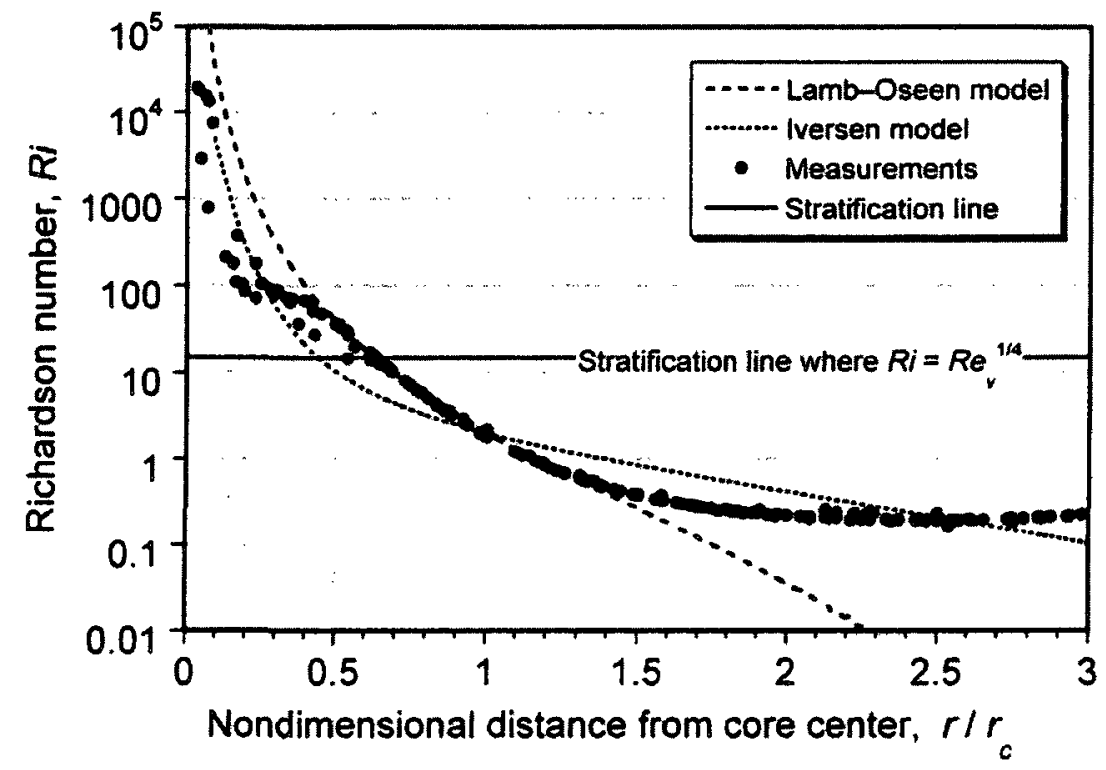

Figure C.2: Local Richardson Number with Radial Coordinate for a Vortex Flow Obtained by Measurements at $\operatorname{Re}_{v}=50,000$ (Leishman, 2006)

Figure $\mathrm{C} .2$ shows that as the radial distance is increased, $R i$ decreases and falls below the stratification threshold, which creates the transitional and fully turbulent zones in Figure C.1. Furthermore, for $R i>(R i)_{t h r e s h}$, only laminar flow is possible in this region. Diffusion occurs at the molecular level since it is the only means of momentum transport within the vortex flow. However, when $R i<(R i)_{t h r e s h}$, turbulent flow within the vortex may develop. This concept explains the persistence of lift-generated tip vortices to relatively old wake ages. 